\title{
Evaluation of Damaged Concrete Members Strengthened with CFRP-Epoxy and HFRP Polyurethane Systems
}

Krishna Tulasi Gadde

Follow this and additional works at: https://researchrepository.wvu.edu/etd

\section{Recommended Citation}

Gadde, Krishna Tulasi, "Evaluation of Damaged Concrete Members Strengthened with CFRP-Epoxy and HFRP Polyurethane Systems" (2017). Graduate Theses, Dissertations, and Problem Reports. 5635.

https://researchrepository.wvu.edu/etd/5635

This Thesis is protected by copyright and/or related rights. It has been brought to you by the The Research Repository @ WVU with permission from the rights-holder(s). You are free to use this Thesis in any way that is permitted by the copyright and related rights legislation that applies to your use. For other uses you must obtain permission from the rights-holder(s) directly, unless additional rights are indicated by a Creative Commons license in the record and/ or on the work itself. This Thesis has been accepted for inclusion in WVU Graduate Theses, Dissertations, and Problem Reports collection by an authorized administrator of The Research Repository @ WVU. For more information, please contact researchrepository@mail.wvu.edu. 


\title{
Evaluation of Damaged Concrete Members Strengthened with CFRP-Epoxy and HFRP-Polyurethane Systems
}

\author{
Krishna Tulasi Gadde
}

Thesis submitted

to the Benjamin M. Statler College of Engineering and Mineral Resources at West Virginia University

\author{
in partial fulfillment of the requirements for the degree of \\ Master of Science in \\ Civil Engineering
}

\author{
P.V. Vijay, Ph.D., P.E., Chair \\ Yoojung Yoon, Ph.D., Co-Chair \\ Radhey Sharma, Ph.D. \\ Department of Civil and Environmental Engineering
}

Morgantown, West Virginia

August 2017

Keywords: Carbon, CFRP, Hybrid, HFRP, Pre-preg, FRP, Wrap, Fabric, Concrete Cylinder, Concrete Beam, Reinforcement, Stiffness, Crack Width, Deflection, Ultimate Load

Copyright 2017 Krishna Tulasi Gadde 


\title{
ABSTRACT \\ Evaluation of Damaged Concrete Members Strengthened with CFRP-Epoxy and HFRP-Polyurethane Systems
}

\author{
Krishna Tulasi Gadde
}

Fiber reinforced polymer (FRP) composite materials offer economical solutions to repair and rehabilitate the aging civil infrastructure at a fraction of the huge replacement cost. Glass and carbon FRP fabrics, bars, and shapes have been field implemented for the construction and rehabilitation of reinforced concrete $(\mathrm{RC})$ structures. This research work aims at evaluating the mechanical and bond properties of Fiber Reinforced Polymer (FRP) composites for concrete structural applications. Both CFRP and Hybrid Carbon/Glass FRP (HFRP) have been investigated for repair and rehabilitation of civil infrastructure constructed of concrete. Concrete cylinders of different compressive strengths were tested with 1, 2, and 3 layers of confining CFRP and HFRP wraps. Similarly, split-bonded cylinders with adhesive bonding representing cracked and repaired concrete columns were also tested. In addition to cylinder testing, CFRP and HFRP wrapped concrete beams with varying types of damage were tested to find the wrap effectiveness, structural behavior, and flexural strength gains. Analytical modeling results for both concrete cylinders and beams were compared with the experimental results. Results show that both the fabric systems are effective in providing the necessary bond strength, confinement effects and flexural strength enhancement. 


\section{ACKNOWLEDGEMENTS}

I would like to express my sincere gratitude to my advisor Dr. P. Vijay for giving me the opportunity to study as a graduate student at WVU. His support, guidance and motivation during the course of my Master's education and research work were invaluable.

I am also extremely thankful for all the support provided by Dr. Yoojung Yoon, my co-advisor. I sincerely appreciated his kindness and help provided whenever needed. I am also grateful to Dr. Radhey Sharma for being a member of my review committee and helping me throughout my stay here at WVU.

I am thankful to NRI inc. for funding this research and providing FRP materials needed for carrying out this research work. Also my sincere thanks to Ms. Eri Vokshi of NRI inc. for her support and patience. Supply of concrete repair materials from Sika Corporation is also sincerely acknowledged.

I am also thankful to Mark Skidmore for teaching me to set up instruments and acquire data from them. My sincere thanks to Jerry Nestor and David Turner for all their invaluable help in lab work throughout my research work, without their help I cannot think of completing this work in time. Help provided by student workers Maneesh Chandu Jasti, Sebastian, and Luis Parra is very much appreciated.

I am very thankful to all my colleagues Piyush Soti, Venkat Dheeraj, Mariam Albaghli, Anudeep Paraitham, Kumar Venkatesh, Jonas Kavi, and Praveen Majjigapu who helped me throughout my research work. I would like to thank my friends who have been incredibly supportive to me.

Last but not least, my heartfelt thanks to my husband and my family for their love, understanding, motivation and support provided throughout without them it would be impossible to reach my goals.

Finally, I would like to express my deepest gratitude to West Virginia University and my friends for the lovely experience at Morgantown. 


\section{TABLE OF CONTENTS}

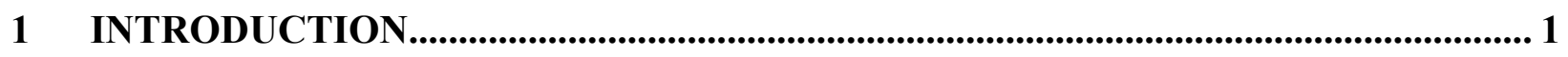

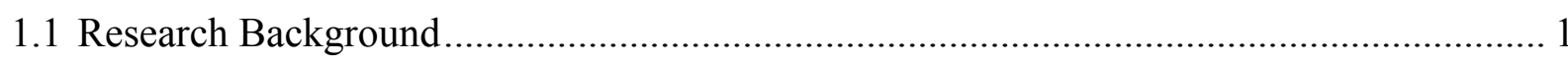

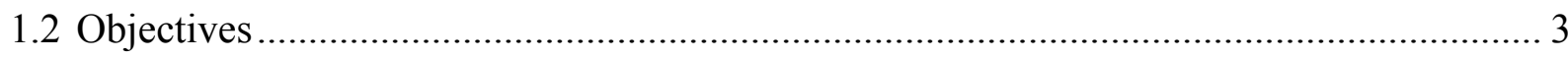

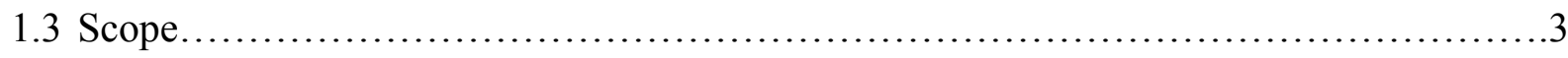

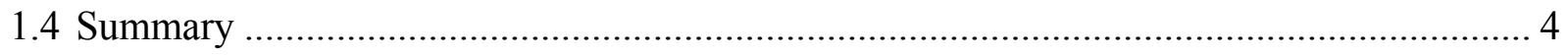

2 LITERATURE REVIEW ............................................................................................ 6

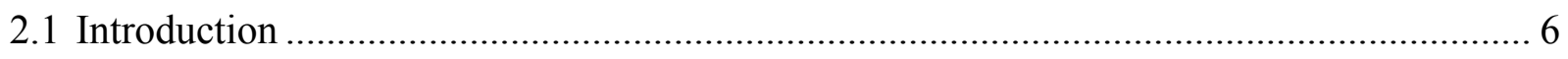

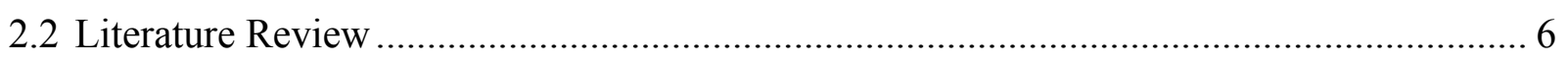

2.2.1 Fiber Reinforced Polymers ……………….................................................... 6

2.2.2 Concrete Cylinders Confinement with CFRP and HFRP ……………………..... 7

2.2.3 Reinforced Concrete Beams ……................................................................. 10

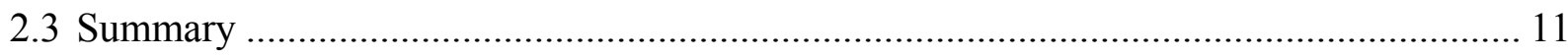

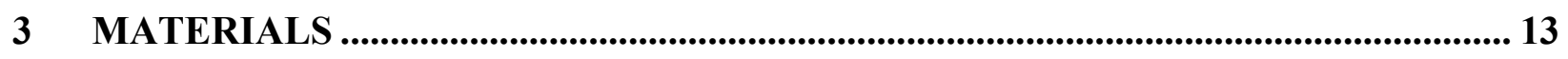

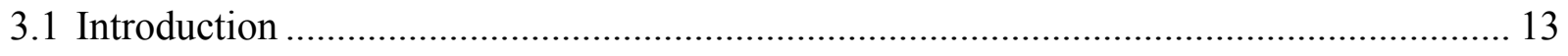

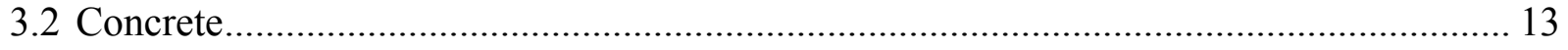

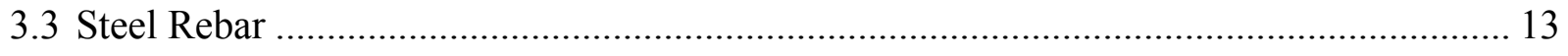

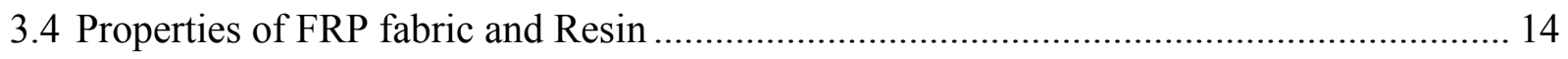

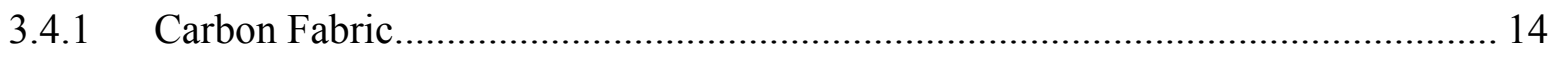




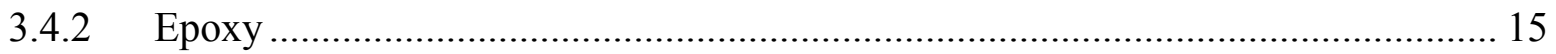

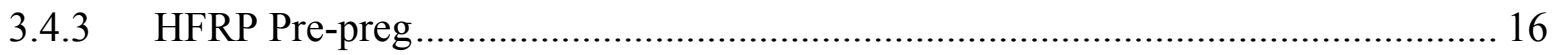

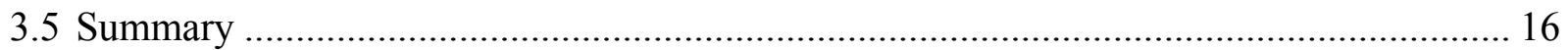

4 TENSION TESTS ON FRP MATERIALS ................................................................ 17

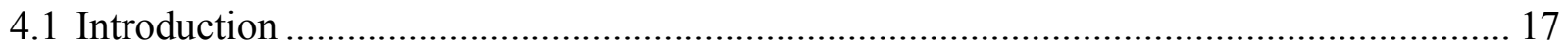

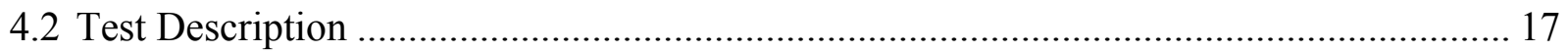

4.2.1 Tension Tests on FRP Coupons ....................................................................... 17

4.2.2 Direct Tension Test (Pull-off Method) ……….................................................. 17

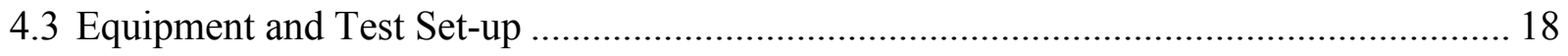

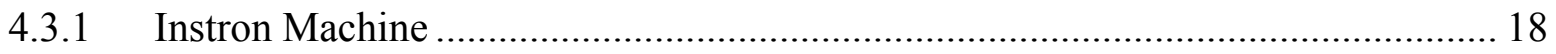

4.3.2 Proceq-DYNA Z16 Pull-off Tester.................................................................. 18

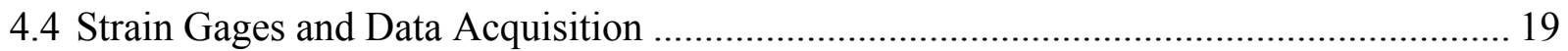

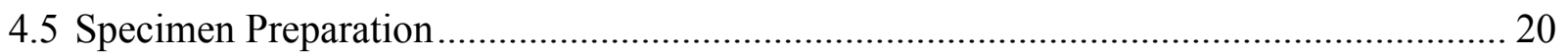

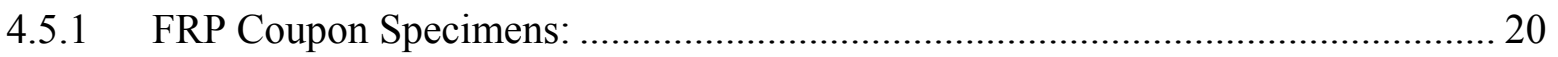

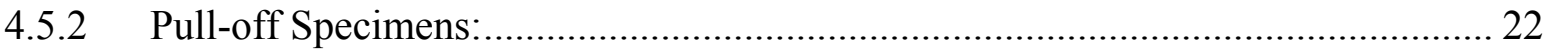

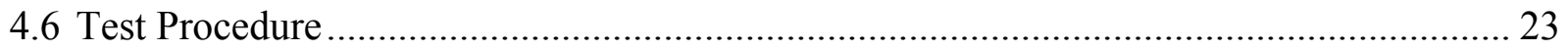

4.6.1 FRP Coupon Specimens: ................................................................................ 23

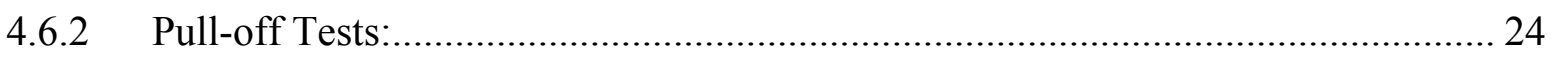

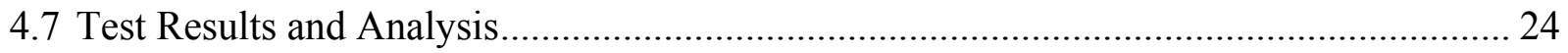

4.7.1 Tension Tests on CFRP-Epoxy Coupons ……….............................................. 24 
4.7.2 Tension Tests on Hybrid pre-preg Coupons .................................................... 26

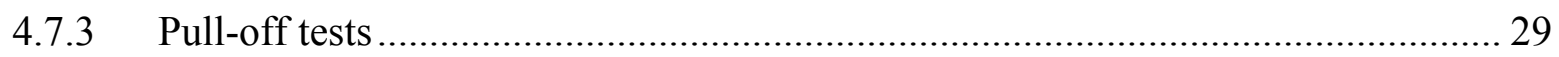

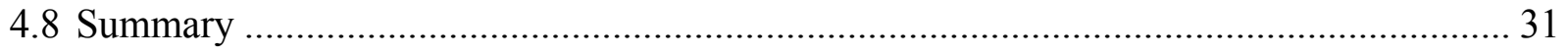

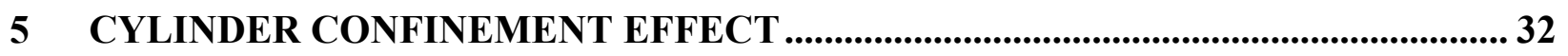

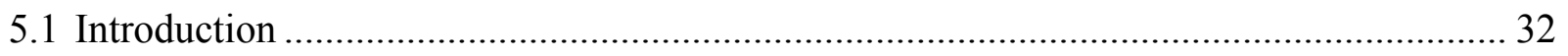

5.2 Compression Tests on Concrete Cylinders ............................................................ 32

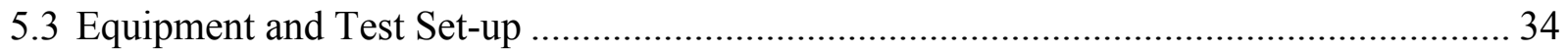

5.3.1 Universal Testing Machine (UTM) ........................................................ 34

5.3.2 Sulfur Capping Compound …................................................................ 35

5.3.3 Strain Gages and Data Acquisition ............................................................... 36

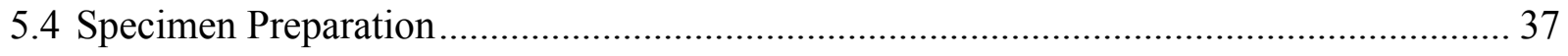

5.4.1 Casting and Curing of Concrete Cylinders …............................................ 37

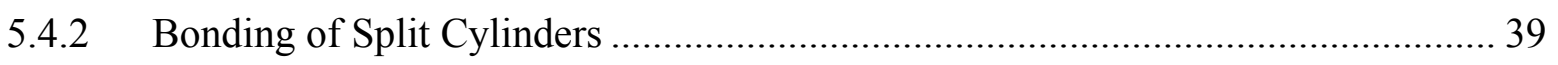

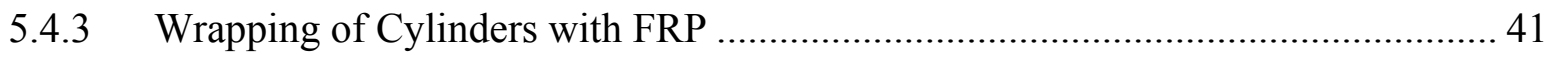

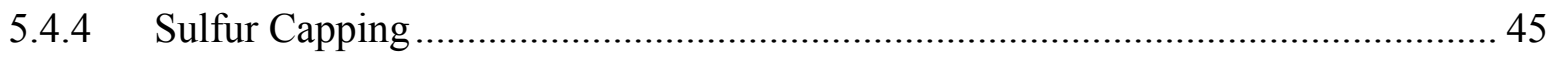

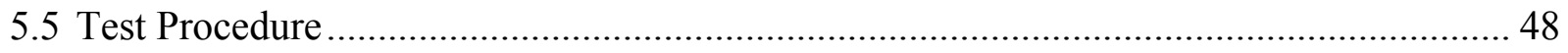

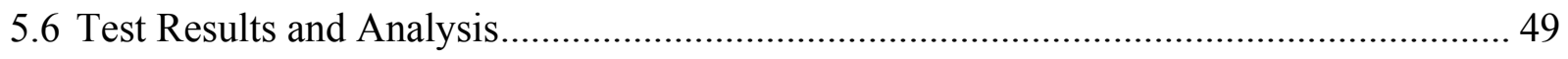

5.6.1 Compression Tests on Full Cylinders .................................................... 50

5.6.2 Stress-Strain Curves of Full Cylinders ....................................................... 54

5.6.3 Theoretical Analysis of Full Cylinders ........................................................... 54 


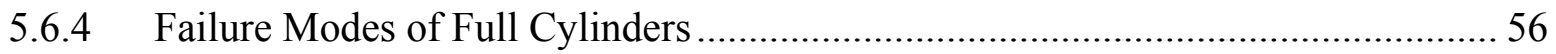

5.6.5 Compression Tests on Split-Bonded Cylinders ...................................................... 60

5.6.6 Stiffness/ Stress-Strain Curves of Split-Bonded Cylinders ................................... 64

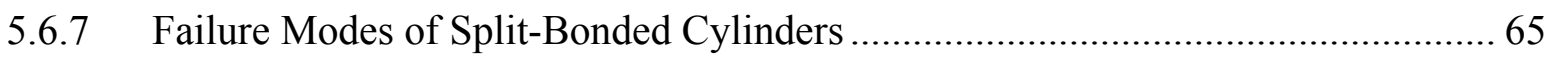

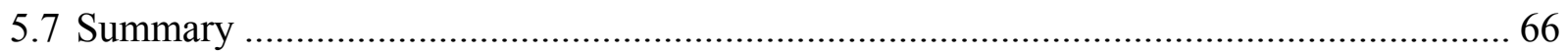

$6 \quad$ RC BEAMS BENDING TESTS...........................................67

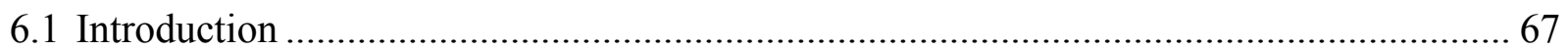

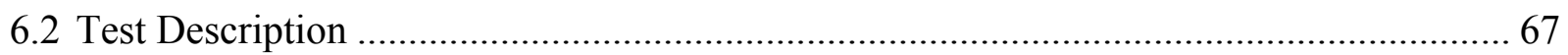

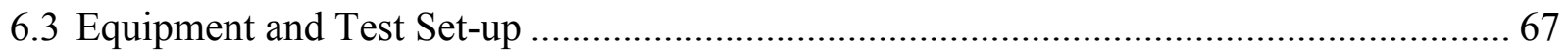

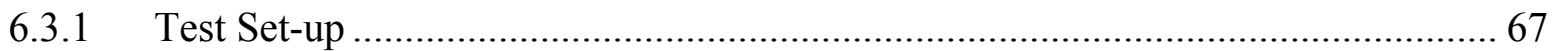

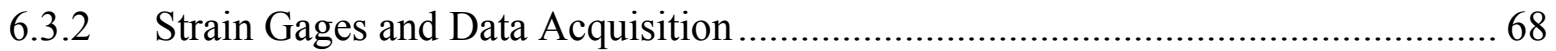

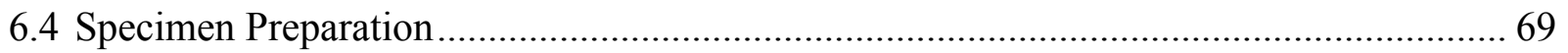

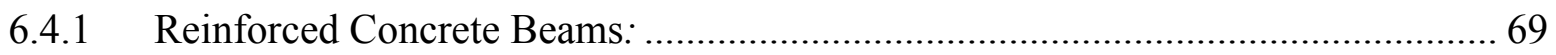

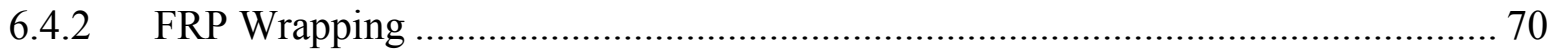

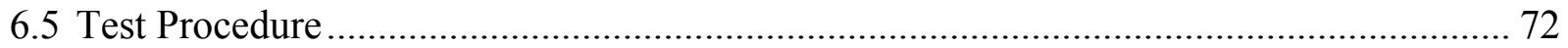

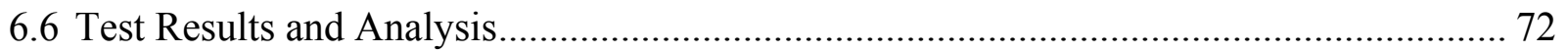

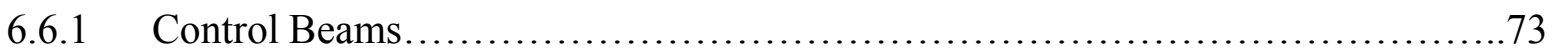

6.6.2 External strengthening of beams with CFRP-Epoxy System .............................74

6.6.3 External strengthening of beams with HFRP Pre-preg ......................................... 77 
6.6.4 Load Deflection Curves .................................................................................. 79

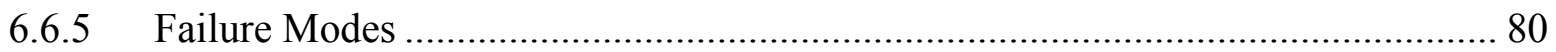

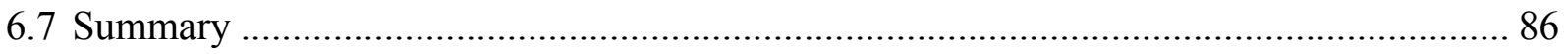

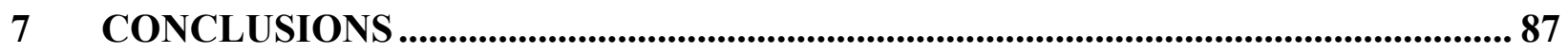

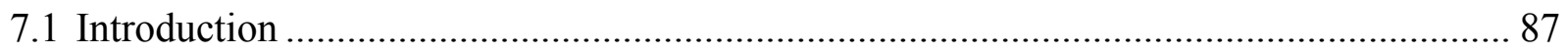

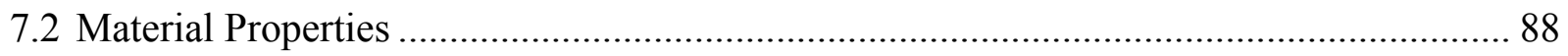

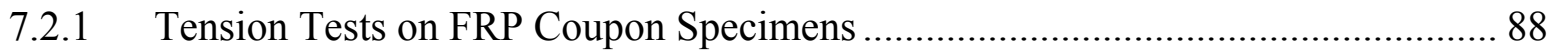

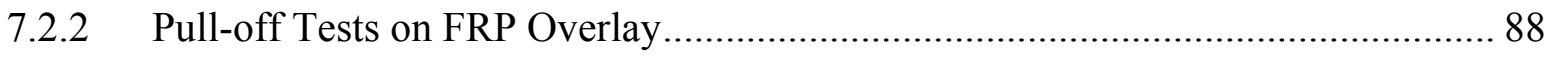

7.3 Compression Tests on Concrete Cylinders ..................................................................... 89

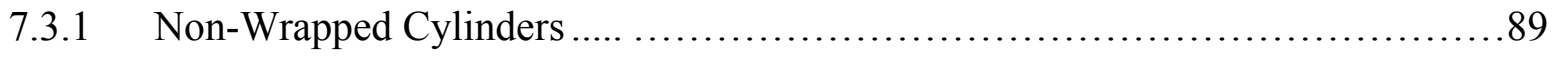

7.3.2 FRP Wrapped Cylinders........................................................ 89

7.4 Flexural Strength Enhancement of RC Beams........................................91

7.4.1 CFRP-Thermopoxy (pre-cracked and wrapped)............................................... 91

7.4.2 HFRP-Polyurethane (pre-cracked and wrapped) .............................92

7.4.4 Effect of Beam Crack Repair through Resin Injection..........................99

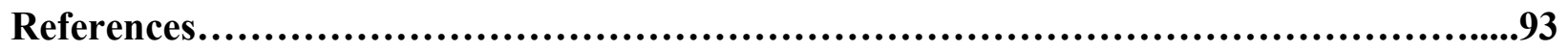

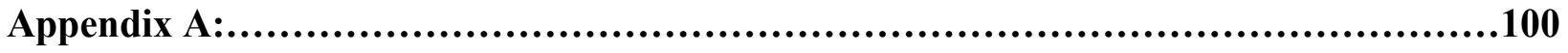

Appendix B: .................................................................................................................................... 103

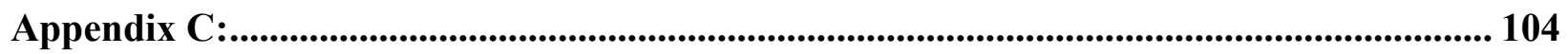

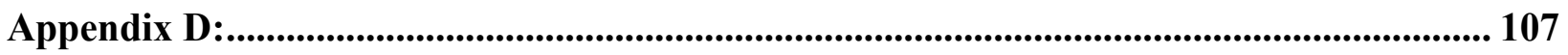


Appendix E: ........................................................................................................................................... 109

Appendix F: ............................................................................................................................................. 117 


\section{LIST of TABLES}

Table 3-1 Mechanical Properties of CFRP Fabric ............................................................. 14

Table 3-2 Mechanical Properties of Epoxy ........................................................................... 15

Table 3-3 Mechanical Properties of HFRP Pre-preg .......................................................................... 16

Table 4-1 Tensile Properties of CFRP-Epoxy Coupons ........................................................ 25

Table 4-2 Tensile Properties of Hybrid Pre-preg Coupons in Carbon Direction ........................... 26

Table 4-3 Tensile Properties of Hybrid Pre-preg Coupons in Glass Direction .............................. 27

Table 4-4 Test Results of Pull-off Tests .......................................................................................... 29

Table 4-5 Test Results of Pull-off Tests with and without Powerwash of Concrete Surface ........ 30

Table 5-1 Compressive Strength of Non-Wrapped Full Cylinders .................................................. 50

Table 5-2 Compressive Strength of CFRP Wrapped Full Cylinders from Batch-1 ..................... 51

Table 5-3 Compressive Strength of CFRP Wrapped Full Cylinders from Batch-2 ..................... 52

Table 5-4 Compressive Strength of HFRP pre-preg Wrapped Full Cylinders from Batch-1........ 53

Table 5-5 Theoretical Analysis of FRP Confined Concrete Cylinders ......................................... 55

Table 5-6 Compressive Strength of Non-Wrapped Split-Bonded Cylinders................................ 61

Table 5-7 Compressive Strength of HFRP Pre-preg Wrapped Split-Bonded Cylinders of Batch-1..

Table 5-8 Compressive Strength of CFRP Wrapped Split-Bonded Cylinders of Batch-2 ........ 63

Table 6-1 Four Point Bending on Control Beams from Batch-1 and Batch-2......................... 74

Table 6-2 Four Point Bending on Externally Strengthened Beams with CFRP-Epoxy System

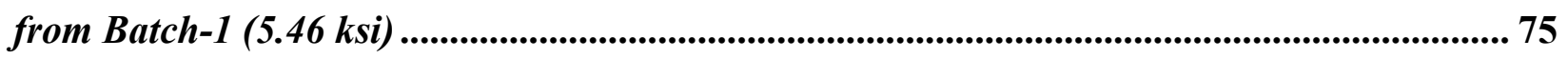

Table 6-3 Four Point Bending on Externally Strengthened Beams with CFRP-Epoxy System

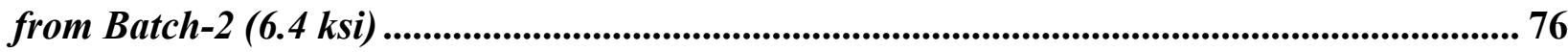


Table 6-4 Four Point Bending on Externally Strengthened Beams with HFRP Pre-preg System

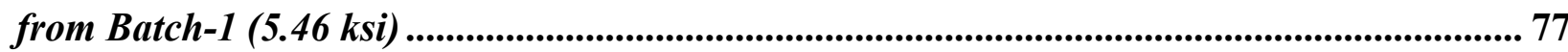

Table 6-5 Four Point Bending on Externally Strengthened Beams with HFRP Pre-preg System

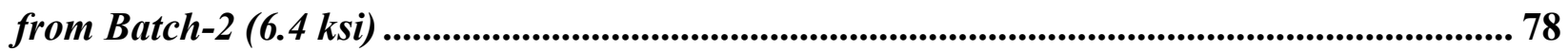

Table D-1 Compressive Strength of HFRP Pre-preg Wrapped Split-Bonded Cylinders of

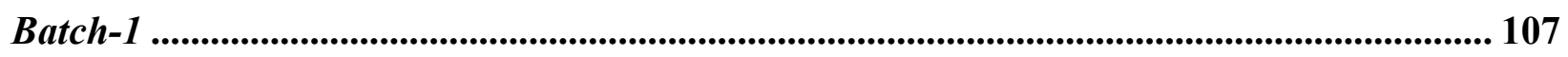

Table D-2 Compressive Strength of CFRP Wrapped Split-Bonded Cylinders of Batch-2 ..... 108 


\section{LIST OF FIGURES}

Figure 2-1 Lateral Expansion (Left) and Effect of Confinement (Right) [5] ..................... 7

Figure 2-2 Free Body Diagram of Section of Confined Concrete [5] ............................... 7

Figure 2-3 FRP Wrapping: (a) Single type; (b) Two type; (c) Three type [4].................... 9

Figure 2-4 Vertical cracks in column ....................................................................... 9

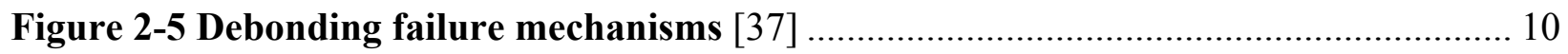

Figure 2-6 Figure fabricated carbon-glass HFRP sheets (black CF rovings, white GF rovings)

$[40]$

Figure 2-7 Bi-directional Carbon-Glass Hybrid Fabric.............................................. 12

Figure 3-1 Unidirectional Carbon Fabric ........................................................... 14

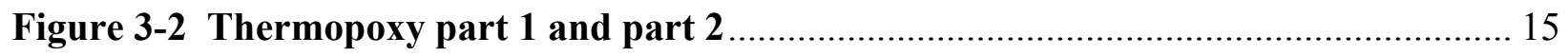

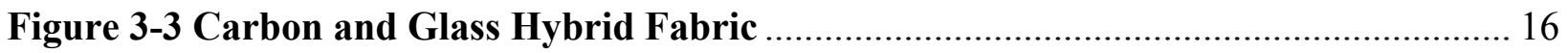

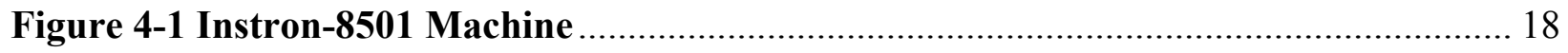

Figure 4-2 Proceq-DYNA Z16 Pull-off Tester................................................................ 19

Figure 4-3 (a) 350 Ohm Strain Gages (b) Data Acquisition System-8000 (c) 350 Ohm long

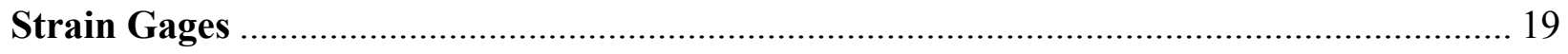

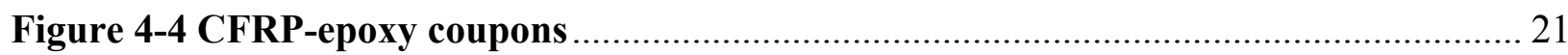

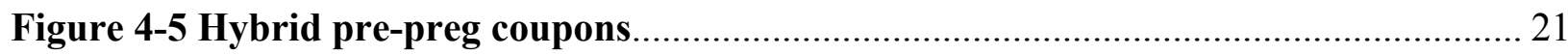

Figure 4-6 FRP overlay on concrete substrate $(1,2$, and 3 layers)................................. 22

Figure 4-7 Drilling 2" diameter notches on the test specimen ..................................... 22

Figure 4-8 Test specimen with 2" diameter notches ................................................. 22

Figure 4-9 Attaching Aluminum dolly on the surface of FRP notch .............................. 23

Figure 4-10 (a) Testing of CFRP Coupon (b) Testing of HFRP Pre-preg Coupon.............. 23 
Figure 4-11 (a) Pull-off testing (b) Samples of failed specimens.....

Figure 4-12 Tension Tested CFRP-Epoxy Coupons ...................................................... 25

Figure 4-13 Stress-Strain Curve of Single Layer CFRP-Epoxy Coupon (Axial gage) ........ 26

Figure 4-14 Tension Tested Hybrid pre-preg Coupons Oriented in Carbon Direction ....... 27

Figure 4-15 Tension Tested Hybrid pre-preg Coupons Oriented in Glass Direction........... 28

Figure 4-16 Stress-Strain Curve of Three Layer Hybrid pre-preg Coupon Oriented in

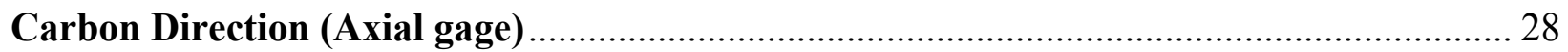

Figure 4-17 Stress-Strain Curve of Three Layer Hybrid pre-preg Coupon Oriented in

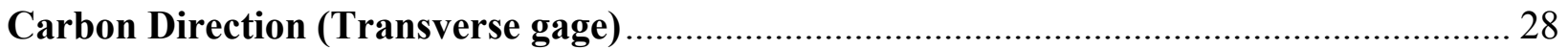

Figure 4-18 Stress-Strain Curve of Three Layer Hybrid pre-preg Coupon Oriented in Glass

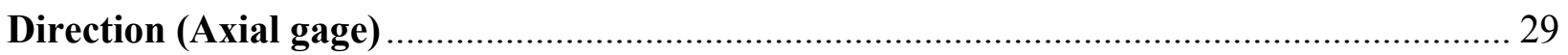

Figure 4-19 Pull-off tests on 1,2, and 3 layers of CFRP-Epoxy and HFRP Prepreg Overlay materials 30

Figure 5-1 Full Cylinders: (a) Non-Wrapped Cylinders (b) CFRP Wrapped Cylinders (c)

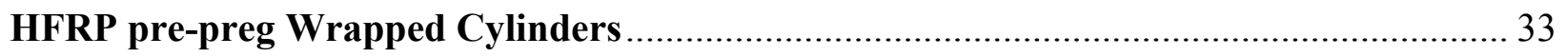

Figure 5-2 Split-Bonded Cylinders: (a) Non-Wrapped Cylinders (b) CFRP Wrapped Cylinders (c) HFRP pre-preg Wrapped Cylinders ................................................... 34

Figure 5-3 Universal Testing Machine (350 kip Capacity) ............................................ 35

Figure 5-4 Universal Testing Machine (500 kip Capacity) ......................................... 35

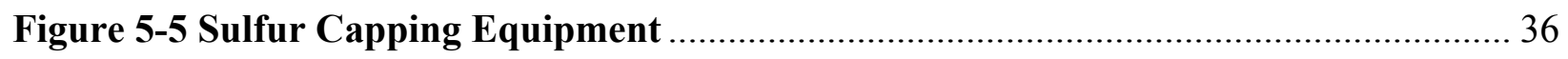

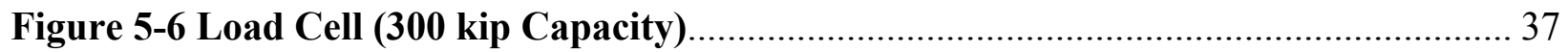

Figure 5-7 Plastic Molds with Plastic Sheets for Casting Split Cylinders ......................... 38

Figure 5-8 Split Cylinders after Curing for 28 Days ................................................ 39 


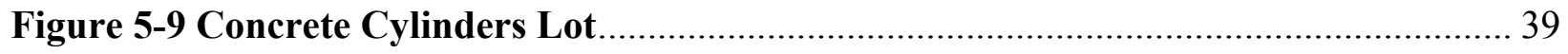

Figure 5-10 Split Cylinders with Applied Concrete Adhesive ......................................... 40

Figure 5-11 Split Cylinders with Hose Clamps ............................................................ 40

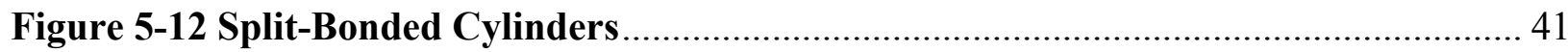

Figure 5-13 Cutting CFRP Fabric into Wraps............................................................ 41

Figure 5-14 Mixing of Two-Part Epoxy (left); Mixed Resin (Right) ................................ 42

Figure 5-15 Saturating CFRP Fabric with Mixed Epoxy .......................................... 42

Figure 5-16 Applying Resin Primer Coat on the Surface of Cylinder ............................. 42

Figure 5-17 Wrapping of Concrete Cylinders with CFRP-Epoxy System ........................ 43

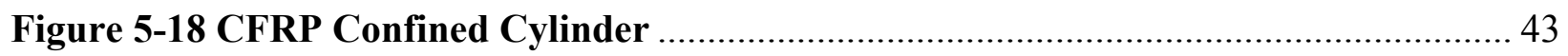

Figure 5-19 Cutting Hybrid pre-preg Fabric into Wraps ............................................. 43

Figure 5-20 Mixing of Two-Part Primer (Left); Mixed Primer (Right) ........................... 44

Figure 5-21 Surface of Concrete Cylinder Coated with Primer ...................................... 44

Figure 5-22 Wrapping of Concrete Cylinders with HFRP pre-preg ................................ 44

Figure 5-23 HFRP pre-preg Confined Cylinders..................................................... 45

Figure 5-24 Molten Sulfur Capping Compound ….................................................. 45

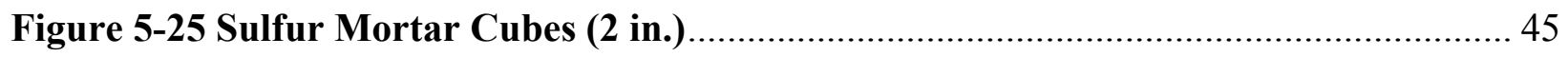

Figure 5-26 Compression Test on Sulfur Mortar Cube ................................................ 46

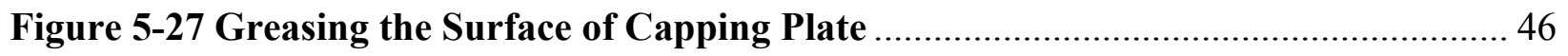

Figure 5-28 Pouring Sulfur Mortar into Capping Plate.............................................. 47

Figure 5-29 Aligning Cylinder against Vertical Device............................................ 47

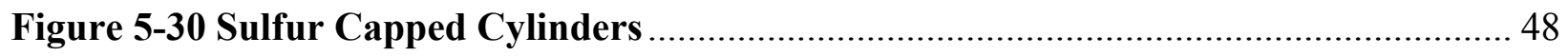

Figure 5-31 Axial Compression Testing of Concrete Cylinders ...................................... 49 
Figure 5-32 Average Axial Strength Increase for 1, 2, \& 3 Layers of Wrapping with CFRP

Figure 5-33 Average Axial Strength Increase for 1, 2, \& 3 Layers of Wrapping with HFRP pre-preg. 53

Figure 5-34 Stress-Strain Curves for Non-Wrapped Full Cylinders, 1Layer CFRP and HFRP

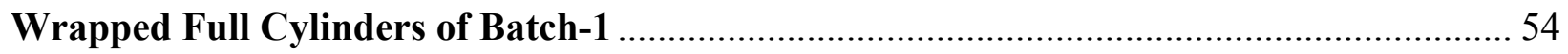

Figure 5-35 Failure Modes of Non-Wrapped Full Cylinders from Batch-1 ...................... 57

Figure 5-36 Failure Modes of 1 Layer CFRP Wrapped Full Cylinders from Batch-1 ........ 57

Figure 5-37 Failure Modes of 2 Layer CFRP Wrapped Full Cylinders from Batch-1 ....... 57

Figure 5-38 Failure Modes of 3 Layer CFRP Wrapped Full Cylinders from Batch-1 ....... 58

Figure 5-39 Failure Modes of 1 Layer HFRP Pre-preg Wrapped Full Cylinders from Batch1. 58

Figure 5-40 Failure Modes of 2 Layer HFRP Pre-preg Wrapped Full Cylinders from Batch1. 58

Figure 5-41 Failure Modes of 3 Layer HFRP Pre-preg Wrapped Full Cylinders from Batch1. 59

Figure 5-42 Failure Modes of Non-Wrapped Full Cylinders from Batch-2 ...................... 59

Figure 5-43 Failure Modes of 1 Layer CFRP Wrapped Full Cylinders from Batch-2 ........ 59

Figure 5-44 Failure Modes of 2 Layer CFRP Wrapped Full Cylinders from Batch-2 ........ 60

Figure 5-45 Failure Modes of 3 Layer CFRP Wrapped Full Cylinders from Batch-2 ........ 60

Figure 5-46 Average Axial Compressive Strength of Non-Wrapped Full and Split-Bonded Cylinders of Batch-1 and Batch-2 
Figure 5-47 Average Axial Compressive Strength of Two Layer Wrapped Full Cylinders and Wrapped Split-Bonded Cylinders of Batch-1 and Batch-2 ............................................ 63

Figure 5-48 Comparison between Non-Wrapped and 2 layer HFRP Pre-preg Wrapped Split-

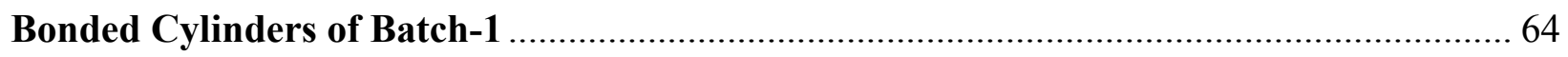

Figure 5-49 Comparison between Full and Split-Bonded Cylinders of Batch-1 ................ 64

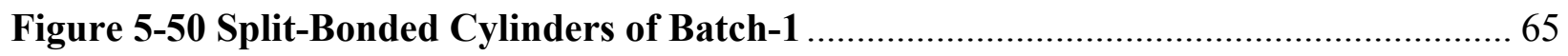

Figure 5-51 Two Layer HFRP Pre-preg Wrapped Split-Bonded Cylinders of Batch-1 ...... 65

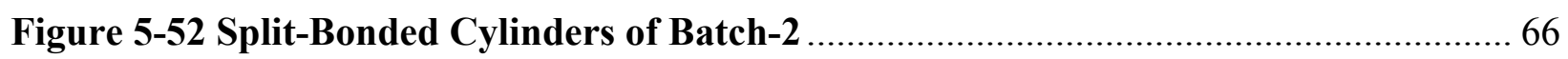

Figure 5-53 Two Layer CFRP Wrapped Split-Bonded Cylinders of Batch-2 .................. 66

Figure 6-1 Test Set-up for Four Point Bending Tests on RC Beams ........................................ 68

Figure 6-2 Making of Steel Rebar Cage.................................................................................. 69

Figure 6-3 Wooden Formwork with Rebar Cage in Position .............................................. 70

Figure 6-4 Application of Primer (Left Side), Beam Wrapping with FRP (Right Side).......70

Figure 6-5 (a) Jack Hammer; (b)Side View; (c) Top View; (d) Mortar Repair.................... 71

Figure 6-6 Load-Deflection Curves for CFRP and HFRP Wrapped Beams of Batch-1...... 79

Figure 6-7 Failure of Control Beam from Batch-1 .................................................................. 83

Figure 6-8 Failure of Control Beam-1 from Batch-2 .................................................... 83

Figure 6-9 Failure of Control Beam-2 from Batch-2 .................................................... 83

Figure 6-10 Failure of 2 Layered CFRP Wrapped Beam-1 from Batch-1 ........................... 84

Figure 6-11 Failure of 2 Layered CFRP Wrapped Beam-2 from Batch-1 ............................ 84

Figure 6-12 Failure of 2 Layered CFRP Wrapped Beam from Batch-2 .............................. 84

Figure 6-13 Failure of 3 Layered CFRP Wrapped Beam from Batch-2 .............................. 85

Figure 6-14 Failure of 2 Layered HFRP Wrapped Beam-1 from Batch-1 ........................... 85 
Figure 6-15 Failure of 2 Layered HFRP Wrapped Beam-2 from Batch-1 .............................. 85

Figure 6-16 Failure of 2 Layered HFRP Wrapped Beam from Batch-2 ............................... 86

Figure 6-17 Failure of 3 Layered HFRP Wrapped Beam from Batch-2 ............................ 86

Figure A-1 Actual stress-strain curves for non-prestressed steel bar reinforcement (Grades 75,60 , and 40, top to bottom) that were manufactured during the mid-1960s..............100

Figure A-2 Comparison of two commercially available high strength reinforcing materials

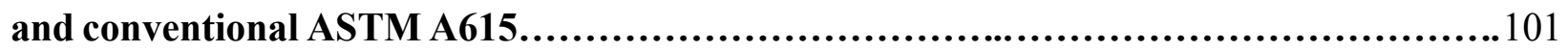

Figure A-3 Stress-Strain Relationships for Grade 60 US bars ......................................... 101

Figure A-4 Stress-Strain Curves for ASTM Grade 60 Steel bars ...................................... 102

Figure A-5 Stress-Strain Curves for ASTM Grade 60 and 80 Steel bars............................. 102

Figure C-1 Stress-Strain Curves for Non-Wrapped Full Cylinders, 1, and 2 Layer HFRP

Pre-preg Wrapped Full Cylinders of Batch-1...........................................104

Figure C-2 Stress-Strain Curves for Non-Wrapped Full Cylinders, 1, 2 and 3 Layer CFRP

Wrapped Full Cylinders of Batch-1 .................................................................................. 104

Figure C-3 Stress-Strain Curves for Non-Wrapped Full Cylinders, 1, 2 and 3 Layer CFRP

Wrapped Full Cylinders of Batch-2 ............................................................................................. 105

Figure C-4 Stress-Strain Curve for Non-Wrapped Split-Bonded Cylinders of Batch-1.... 105

Figure C-5 Stress-Strain Curve for Non-Wrapped Split-Bonded Cylinders of Batch-2.... 106

Figure C-6 Stress-Strain Curve for CFRP Wrapped Split-Bonded Cylinders of Batch-2 106

Figure F-1 Load-Deflection Curves for CFRP wrapped beam of Batch-2: Before Crack Injection v/s After Crack Injection ............................................................................................. 117

Figure F-2 Load-Deflection Curves for HFRP wrapped beam of Batch-2: Before Crack Injection v/s After Crack Injection....................................................... 117 
Figure F-3 Load-Deflection Curves for Control Beam of Batch-1 .......................................... 118

Figure F-4 Load-Deflection Curves for Control Beam-1 of Batch-2 ................................... 118

Figure F-5 Load-Deflection Curves for Control Beam-2 of Batch-2 .................................. 119

Figure F-6 Load-Deflection Curves for CFRP Wrapped Beams of Batch-1..................119

Figure F-7 Load-Deflection Curves for CFRP Wrapped Beams of Batch-2....................... 120

Figure F-8 Load-Deflection Curves for HFRP Wrapped Beams of Batch-1 ...................... 120

Figure F-9 Load-Deflection Curves for HFRP Wrapped Beams of Batch-2 ..................... 121 


\section{INTRODUCTION}

\subsection{Research Background}

There are several thousands of bridges all around US, which are several decades old and in deteriorated condition requiring immediate attention. According to 2017 Report Card for America's Infrastructure (ASCE), "civil engineering infrastructure is rated as " $\mathrm{D}+$ " and requires an investment of $\$ 2$ trillion over next 10 years. Among highway infrastructure, there are several thousand bridges all around US, which are several decades old and are in deteriorated condition requiring immediate attention. The average age of the nation's 614,387 bridges is currently 43 years and $9.1 \%$ of the nation's bridges are rated as structurally deficient. Even though there is an increase in funding for bridges at all levels of government, it was estimated that there is a backlog of $\$ 123$ billion on rehabilitation of bridges". Eliminating bridge deficiency is indispensable due to their enormous effect on nation's economy as road transportation is the most important means of growth of economy by making communication comfortable and cost effective. However, replacing all existing aged and deteriorated bridges is not an easy task. Moreover, it will be a huge burden on government as it is highly expensive. This indicates the need for rehabilitation of the bridges economically is of utmost importance.

Corrosion, materials degradation, and damage during operations are taking a toll on these aging facilities. In addition, the high costs associated with repair and replacement of critical components present many challenges in keeping these infrastructure in safe and durable conditions. With recent material and processing advances, fiber reinforced polymer (FRP) composites offer the great potential for repair, rehabilitation, and replacement of these critical civil infrastructure at a reduced 
cost. Several researchers have done lot of research and proved the efficiency of FRP composites in repair and rehabilitation of structural components. In addition to repair, greater durability can be attained with corrosion resistant FRP components. This research work aims at evaluating the mechanical and bond properties of carbon (CFRP) and carbon/glass hybrid (HFRP) FRP composites for repair and rehabilitation of civil infrastructure.

Glass and carbon FRPs (Fiber Reinforced Polymers) are being increasingly employed for new construction and repair/rehabilitation of in-service civil engineering infrastructure including underwater structures. FRPs offer the advantages of high strength to weight ratio, confinement effects to enhance strength and ductility of underlying substrate, excellent durability including excellent resistance against corrosion, moisture absorption, and chemical attack.

In this project, CFRP and HFRP wraps will be tested at coupon and component level. The CFRP fabrics will be saturated with epoxy resin prior to wrapping on structural members, whereas presaturated C/G-FRP (carbon/glass-FRP) fabric with polyurethane resin is provided for wrapping. Bonding of wraps to structural members is similar to wall paper application and the process is referred to as wet-layup. FRP wraps also act as protective membranes in inhibiting the flow of moisture and air into concrete members, thus reducing the rate of corrosion of steel reinforcement. Following the structural response evaluation of CFRP and C/G-FRP wrap systems with concrete structural members, methodologies can be developed for using them in repair and rehabilitation of damaged concrete members of bridge structures. C/G-FRP wrap system will also be referred to as HFRP. 


\subsection{Objectives}

The main objective of this research is to evaluate the FRP fabrics for rehabilitation of civil infrastructure. As a part of this research, the objectives of evaluation of FRP wraps, concrete cylinders, and beams with CFRP and C/G FRP wraps are as follows:

1) To test FRP coupon specimens for determining the tensile properties such as strength, stiffness, and strain of the fabrics.

2) To determine the pull-off bond strength between concrete surface and FRP.

3) To evaluate the effect of number of layers of FRP wraps on confinement related compressive strength enhancement.

4) To evaluate FRP wraps for repairing damaged (cracked and bonded) concrete cylinders.

5) To evaluate flexural strengthening of damaged reinforced concrete (RC) beams with FRP wrap.

6) To evaluate FRP wraps for repairing of failed reinforced concrete (RC) beams.

\subsection{Scope}

Strain gages were used in all types of tests conducted to obtain load and strain data in order to obtain stiffness of the specimens.

1) To determine the tensile strength, stiffness, and elongation, 60 FRP coupon specimens were prepared as per ASTM D3039 and tested in tension with strain gage attached at mid height in INSTRON machine.

2) FRP wrap was bonded on to the concrete slab and conducted Pull-off test on 43 specimens to determine its bond strength. 
3) Concrete casting was done in two batches. Batch 1 with 5460 psi concrete and Batch 2 with 6400 psi concrete. 6 " x 12" concrete cylinders, 6" x 15" x 120" reinforced concrete beams and 12" x 3" x 36 " concrete slab were cast for two different batches of concrete.

4) Unconfined cylinder specimens were tested under axial compression in UTM and considered as a base line to compare the results of FRP confined cylinder specimens. Confinement of cylinder specimens was carried out with 1,2 and 3 number of layers of CFRP-epoxy and HFRP pre-preg wraps. A total of 39 full cylinders were tested.

5) Twenty two (22) artificially cracked concrete cylinders were cast by using a thin plastic sheet at the center of the cylinder mold. Concrete adhesive was used to bond the split cylinder halves, wrapped with FRP layers and tested in axial compression.

6) Eleven (11) non-wrapped and wrapped RC beams were tested in flexure under four point loading to evaluate the enhancement in flexural strength. Prior to wrapping, plain RC beams were subjected to flexure up to cracking laod.

\subsection{Summary}

This report is organized in to 7 chapters. Each chapter deals with the particular aspect of this research work.

$>$ Chapter 1 gives the introduction to background of the work, objectives, and scope of this research.

$>$ Chapter 2 contains the literature review of the research work done by several other researchers on effect of beam strengthening and confinement of structural elements with FRP wraps.

$>$ Chapter 3 provides a description on the materials used in the entire research work. 
$>$ Chapter 4 summarizes the test results on FRP such as tensile tests on coupons and pull-off tests for obtaining tensile strength and bond strength properties of the FRP materials.

Chapter 5 describes the compressive test results on concrete cylinders for evaluating the confinement effect.

$>$ Chapter 6 focuses on the bending test results of four-point bending tests on RC beams.

$>$ Chapter 7 presents the conclusions and discussions. 


\section{LITERATURE REVIEW}

\section{$2.1 \quad$ Introduction}

In recent times, there is an enormous increase in use of FRPs for structural purposes such as FRP wraps/shells for repair and rehabilitation of aged/deteriorated structures, FRP reinforcing bars for concrete, prefabricated FRP panels for structural elements such as girders, slabs, decks and many others [1]. This chapter describes the overview of the FRP wrapping of concrete structural elements for repair and rehabilitation of the existing aged deteriorated structures.

\subsection{Literature Review}

\subsubsection{Fiber Reinforced Polymers}

Numerous researchers have worked on strengthening of structural components using FRP materials. Advantages of these advanced composite materials are light weight, resistance to corrosion, high strength, good ductility, and resistance to fatigue [2]. The cost of FRPs has decreased remarkably with abundant increase in applications across many fields such as automobile, recreation, sports and aerospace industries. As a consequence of this decrease in cost makes these materials cost effective when compared to traditional construction supplies [3].

Rapid development of FRP technology has been witnessed over recent years. CFRP and GFRP have been the most commonly used fabrics worldwide. Other types of FRP production include AFRP (Aramid FRP), BFRP (Basalt FRP), PBO (Polypara-phenylene-Benzo-bis-Oxazole), DFRP (Dyneema FRP) and HFRP (Hybrid FRP) [4]. HFRP can be formed by combining any two types

of FRP to maximize the benefits from each FRP. In this research work, HFRP, combination of 
carbon and glass FRPs has been used. Higher strength, ductility and cost savings are anticipated with the use of HFRP.

\subsubsection{Concrete Cylinders Confinement with CFRP and HFRP}

Repairing of deteriorated columns using FRP techniques has been widely used in civil infrastructure due its confinement effect. Columns are compression members and FRPs carry high tensile forces. When load is applied on FRP confined column, the compressive force results in circumferential (hoop) expansion of concrete and this in turn causes FRP wrap to expand in tension along hoop direction. Thus, confinement effect of FRP wraps results in enhanced strength, ductility and stiffness of the structural components. The illustration of confinement effect is shown in Fig 2.1 and $2.2[5]$.
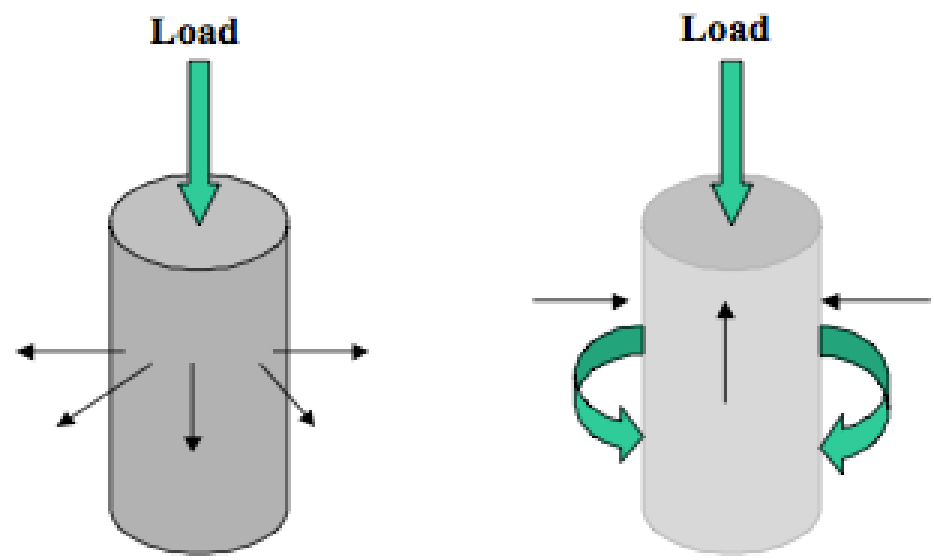

Figure 2-1 Lateral Expansion (Left) and Effect of Confinement (Right) [5]

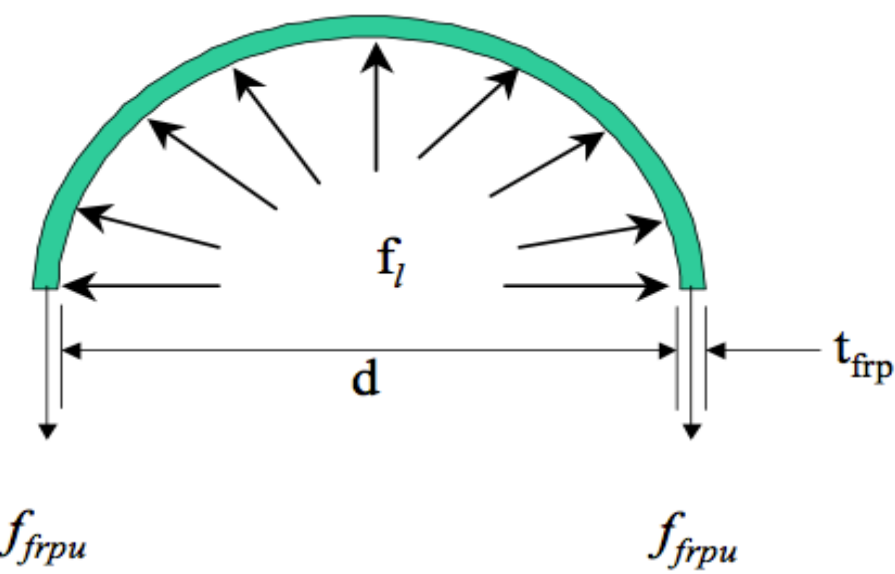

Figure 2-2 Free Body Diagram of Section of Confined Concrete [5] 
The lateral confining pressure $\left(f_{l}\right)$ can be obtained from the equilibrium of forces:

Where:

$$
f_{l}=\frac{f_{f r p u} t_{f r p}}{r}=\frac{2 f_{f r p u} t_{f r p}}{d}
$$

$f_{\text {frpu }}=$ Ultimate tensile strength of circular confined concrete (ksi)

$f_{l}=$ Lateral confining pressure (ksi)

$t_{f r p}=$ Thickness of FRP (inches)

$r=$ Radius of cylinder (inches)

$d=$ Diameter of cylinder (inches)

Over the past three decades, several researchers have worked on evaluating the effectiveness of FRP wraps confining concrete columns or cylinders. The topics of focus include strength enhancement with increase in number of layers/change in fiber orientation $[6,7,8,9]$, effect of different types of FRPs $[10,11,12]$, ageing effects $[13,14,15]$, and impact of environmental conditions $[16,17,1,18]$.

Wei et al. (2009) studied strengthening of partially deteriorated concrete columns with CFRP only near deteriorated part to estimate the influence of a partial confinement [19]. Several empirical confinement models are available [20, 17, 21, 22, 23, 24, 25]. ACI 440.2R-08 model is observed to provide a very good comparison between experimental and theoretical results. ACI equation is given in section 5.6.8.

$\mathrm{Wu}$, et al. (2008) evaluated the confinement effect of concrete cylinders with hybrid FRP wrapping. They obtained hybrid effect by placing one type of FRP layer over another type of FRP as shown in Fig 2.3. Wu, et al. considered a combination of carbon-glass and carbon-aramid fabrics. The study concluded the importance of hybrid ratio for attaining effective and economical hybridization, adding high strength FRP to high ductility FRP helps in improving ultimate strength and the ultimate strain remains same as that of high ductile FRP. Authors have suggested 
analytical models for prediction of failure strength and strain and multi-linear model for prediction of stress-strain relationship [4].

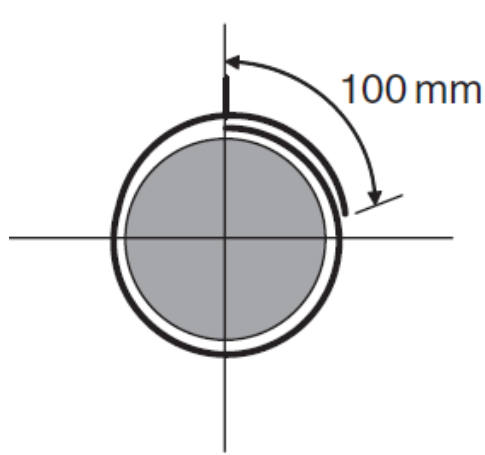

(a)

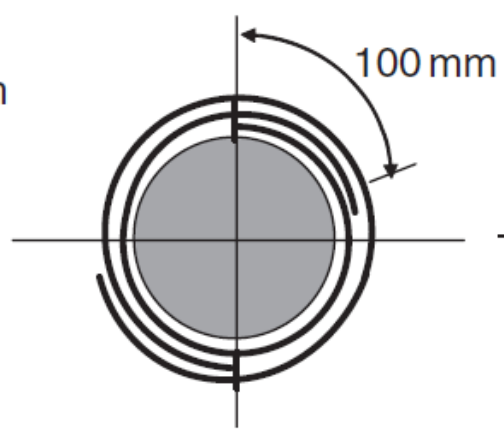

(b)

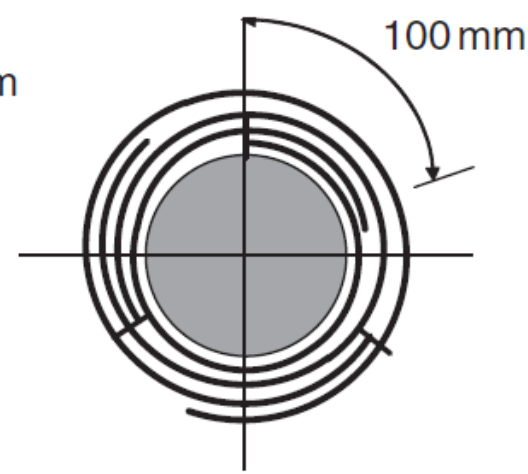

(c)

Figure 2-3 FRP Wrapping: (a) Single type; (b) Two type; (c) Three type [4]

Vertical cracks are observed in concrete columns as shown in a bridge column in a West Virginia bridge pile system (Fig 2.4). This research work will focus on evaluating the use of different types of FRPs for repairing damaged concrete sections as well as damaged FRPs.

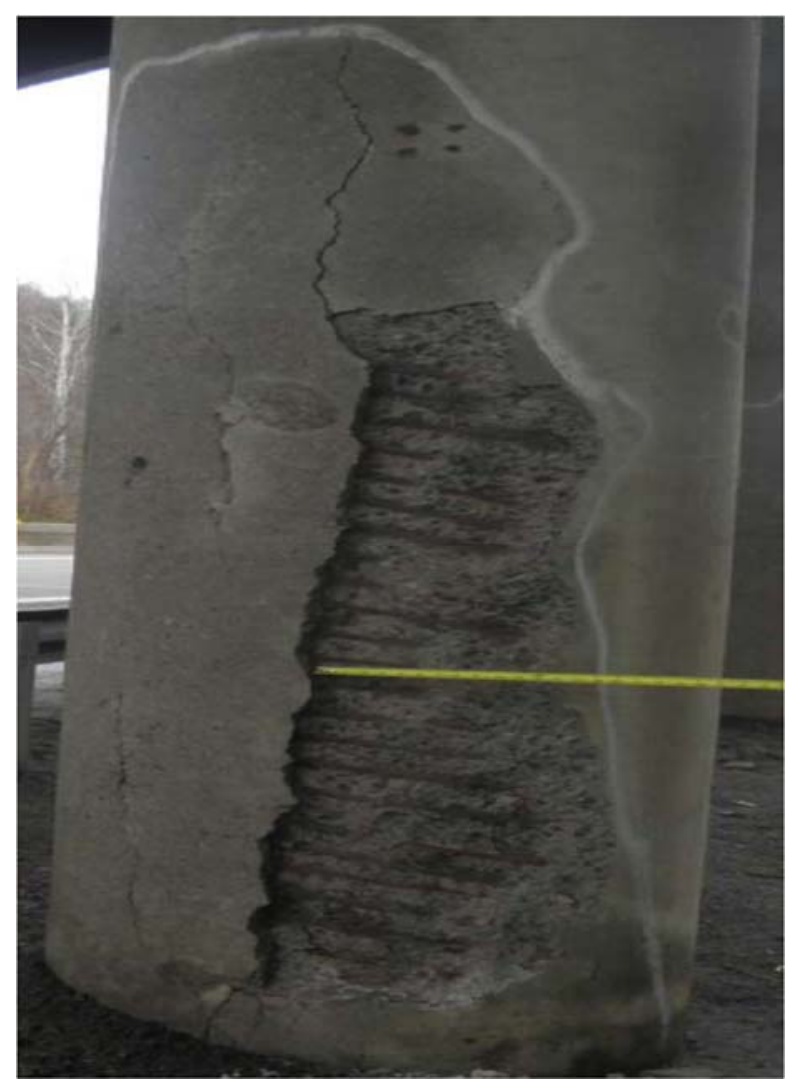

Figure 2-4 Vertical cracks in column (Courtesy: Column from a WVDOH bridge) 


\subsubsection{Reinforced Concrete Beams}

Bending and shear behaviour of FRP strengthened RC beams have been examined by various researchers $[26,27,28,29,30,31,32,33]$. Significant strength and stiffness increase are noted depending on type of FRP, number of FRP layers, bonding adhesives used, surface characteristics, and beam dimensions including area of steel. Some of the predominant failure modes noticed from literature review are concrete crushing, rupture of FRP strips, shear failure, delamination of FRP strips, and concrete cover separation (Fig 2.5) [34, 35, 36, 37, 38].

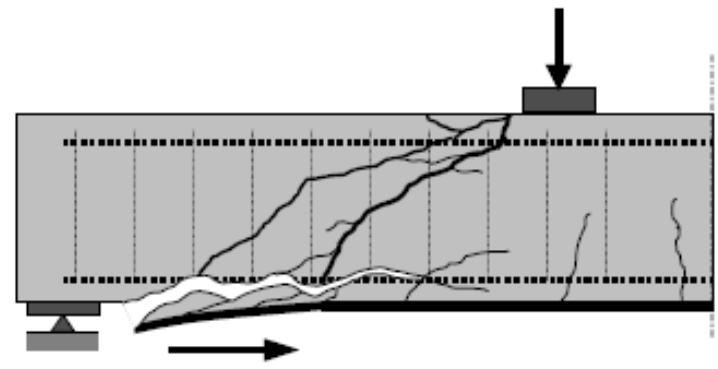

(a) shear failure with concrete cover debonding

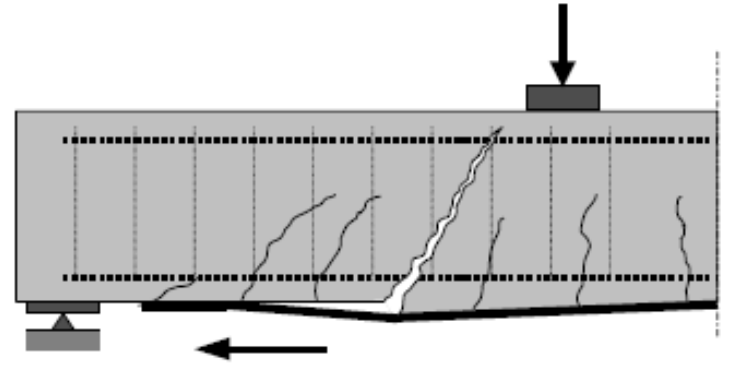

(c) FRP debonding from flexure-shear crack

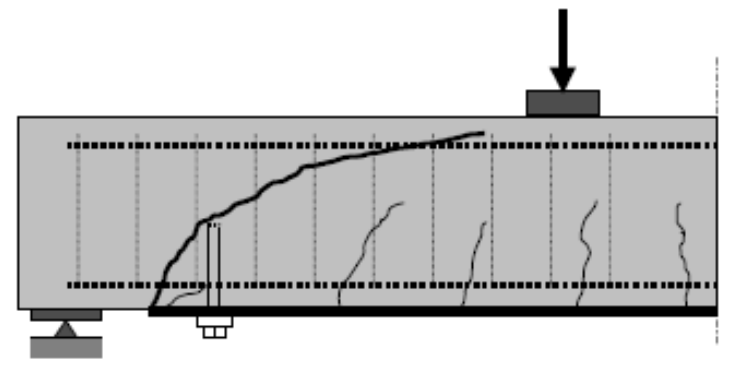

(e) plate end shear failure

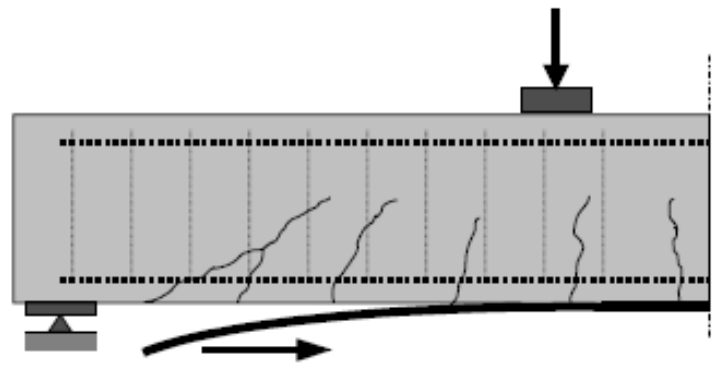

(b) FRP debonding from plate end

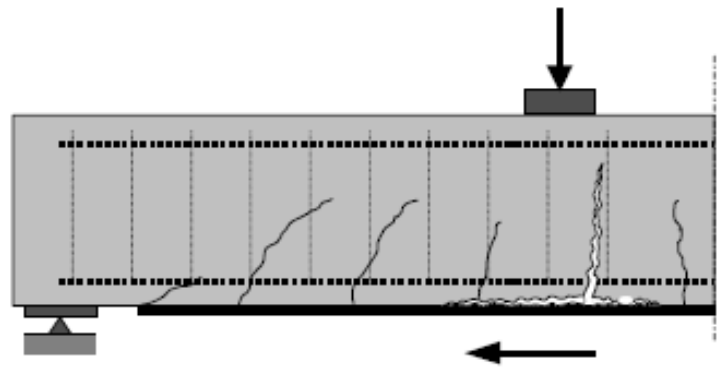

(d) FRP debonding from flexural crack

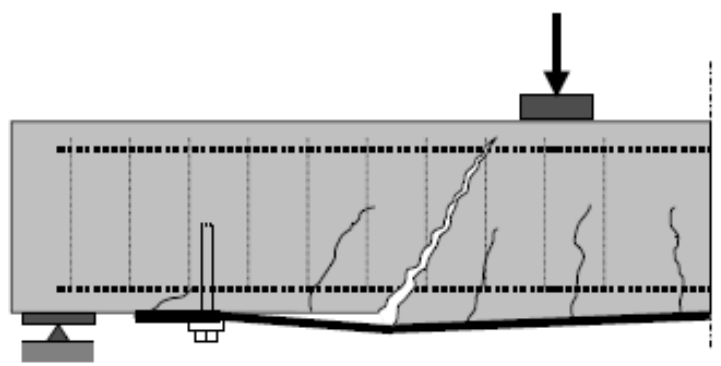

(f) shear failure and debonding

Figure 2-5 Debonding failure mechanisms [37] 
Choi, et al. (2011) and Kang, et al. (2014) investigated the tensile behavior of hybrid FRP with a combination of (carbon/glass) fibers (Fig 2.6) fabricated with a volumetric ratio ranging between $(1 / 1)$ and (10/1) and also tested the effects of strengthening structural concrete members with hybrid FRP. They discovered the higher initial stiffness and an increase in pseudo-ductility even for a very low (carbon/glass) ratio. At rupture, an increase in strain with respect to increasing (carbon/glass) ratios was observed in carbon. Stresses at carbon rupture were fairly close to the predicted values obtained by rule of mixtures while at glass rupture, they were significantly higher. They also concluded that tensile modulus of hybrid FRP is usually consistent with the rule of mixtures, the strains at carbon and glass ruptures on average are substantially higher than their individual ultimate strains. [39, 40]

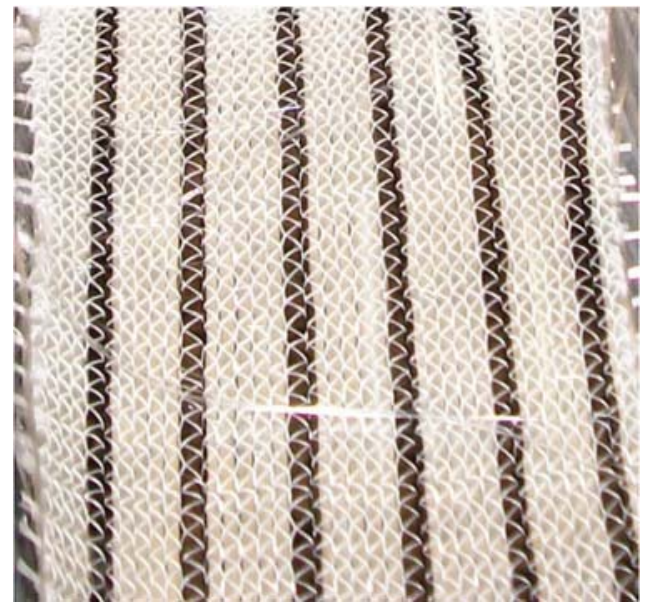

Figure 2-6 Figure fabricated carbon-glass HFRP sheets (black CF rovings, white GF rovings) [40]

\subsection{Summary}

Literature review consisted of experiments on the use of unidirectional hybrid FRPs. Limited or no literature was found for the application of bidirectional hybrid FRP (Fig 2.7) with carbon in longitudinal and glass in transverse directions and also information related to repair of damaged 
(split) concrete members and repair of damaged FRP materials. Hybrid fabrics are expected to provide necessary strength, stiffness and confinement in addition to cost effectiveness.

Based on the limited information available in literature on FRP hybrids and CFRP with highviscosity epoxy, this research work will focus on the performance of:

i. CFRP wet layup with high-viscosity epoxy resin

ii. Bidirectional HFRP pre-impregnated with polyurethane resin

The items considered for structural evaluation are:

a. Strengthening of structural components such as circular columns (cylinders) and beams

b. Repairing of split cylinders and

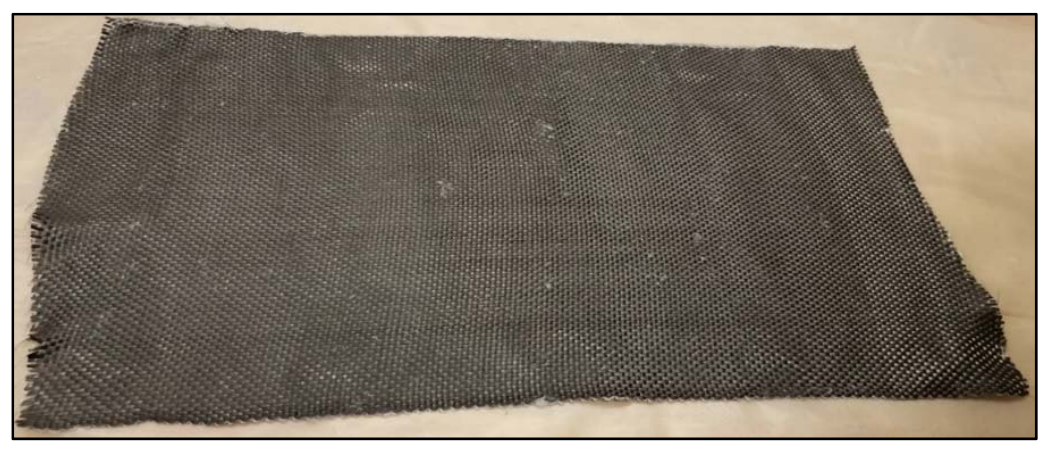

Figure 2-7 Bi-directional Carbon-Glass Hybrid Fabric 


\section{MATERIALS}

\subsection{Introduction}

Material properties play a major role in enhancing the strength of structural systems. Description of all the materials used in this research work and their properties are presented in this chapter.

\subsection{Concrete}

The concrete used in this study was ready-mixed concrete supplied in two batches by Hoy REDIMIX, Morgantown, WV. The compressive strength of concrete of batch I was $5460 \mathrm{psi}$ and that of batch II was 6400 psi. The concrete was poured in the formwork and removed after 24 hours. The concrete cylinders were cured by fully immersing into curing tank filled with water for 28 days. 60 cylinders were cast in each batch. The concrete beams were cured by wet burlap and watered for 28-day curing. Each batch of concrete cast comprised of 6 beams.

\subsection{Steel Rebar}

Steel re-bars were used as internal reinforcement in reinforced concrete beams to provide strength in tension, compression, and shear. Steel re-bars of yield strength 60ksi and diameter of No.3 (3/8”) were adopted as per design calculations in order to attain desired modes of failure in RC beams tested as per wrapping schemes described in chapter 6 . 


\subsection{Properties of FRP fabric and Resin}

\subsubsection{Carbon Fabric}

The carbon fabric consists of unidirectional fiber alignment as shown in Fig. 3.1. The mechanical properties were tabulated in Table 3-1.

Table 3-1 Mechanical Properties of CFRP Fabric

Note: Values from Manufacturer (Conforming to ASTM D3039 and ACI 440.8-13)

\begin{tabular}{|c|c|c|c|}
\hline \multirow{2}{*}{ Property } & Dry fiber & \multicolumn{2}{|c|}{ Laminate } \\
\cline { 2 - 4 } & Typical Test Value & Design Value & Typical Test Value \\
\hline $\begin{array}{c}\text { Tensile Strength } \\
\text { (psi) }\end{array}$ & 550,000 & 152,280 & 204,900 \\
\hline $\begin{array}{c}\text { Tensile Modulus } \\
\text { (psi) }\end{array}$ & $34.5 \times 10^{6}$ & $12.38 \times 10^{6}$ & $12.38 \times 10^{6}$ \\
\hline \begin{tabular}{c} 
Elongation at break \\
\hline $\begin{array}{c}\text { Laminate Thickness } \\
\text { (in.) }\end{array}$
\end{tabular} & $1.5 \%$ & $1.2 \%$ & $1.66 \%$ \\
\hline
\end{tabular}

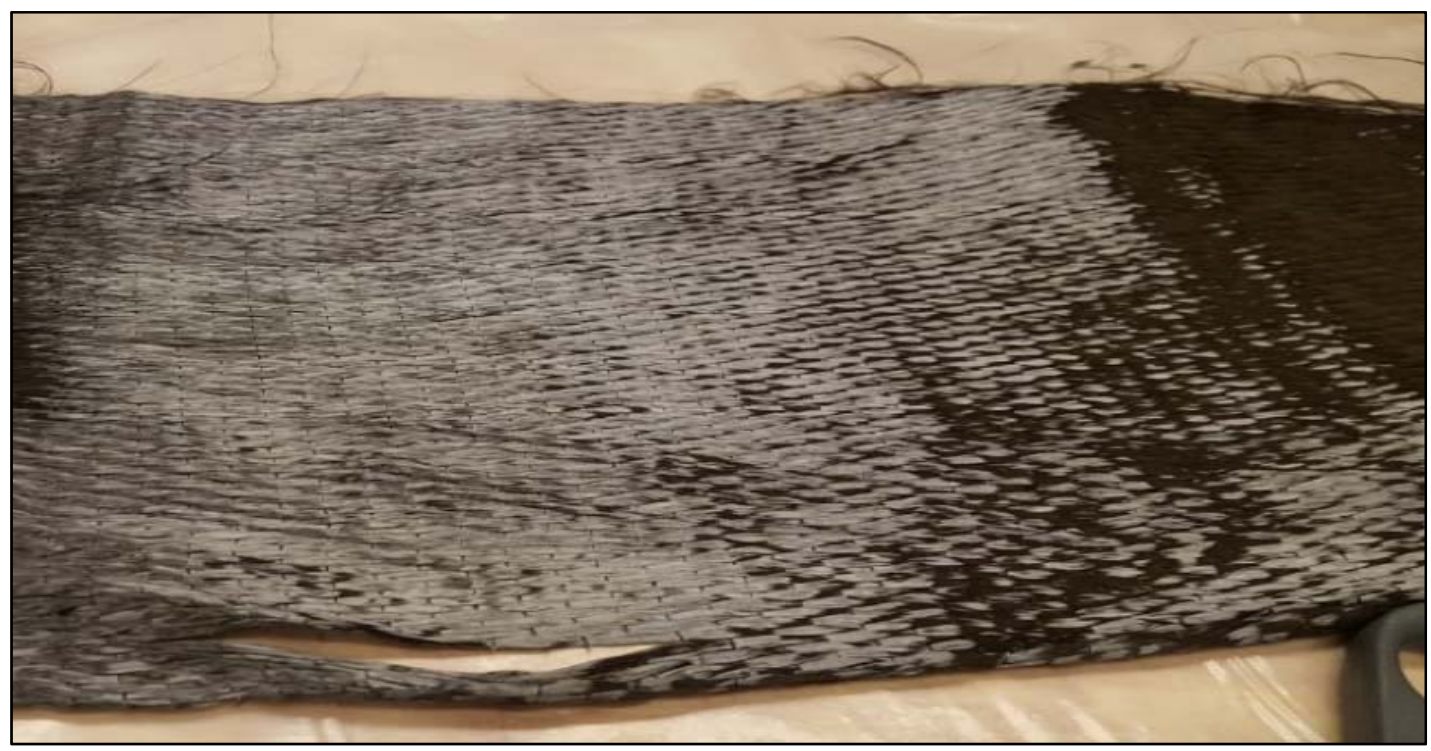

Figure 3-1 Unidirectional Carbon Fabric 


\subsection{2 Ероху}

Resin is a two-part epoxy (Figure 3-2) compound. This can be used as a coating for corrosion resistance or as a saturant for fiberglass or carbon fabrics. The mechanical properties are shown in Table 3-2. Mix ratio of part A to part B is 100: 14.74 by weight.

Table 3-2 Mechanical Properties of Epoxy Note: Values from Manufacturer (Conforming to ASTM D638)

\begin{tabular}{|c|c|}
\hline Property & Typical Test Value \\
\hline Tensile Strength (psi) & 9,500 \\
\hline Tensile Modulus (psi) & 330,000 \\
\hline Viscosity of Part A & $\sim 98,000 \mathrm{cP}$ \\
\hline Viscosity of Part B & $44 \mathrm{cP}$ \\
\hline
\end{tabular}

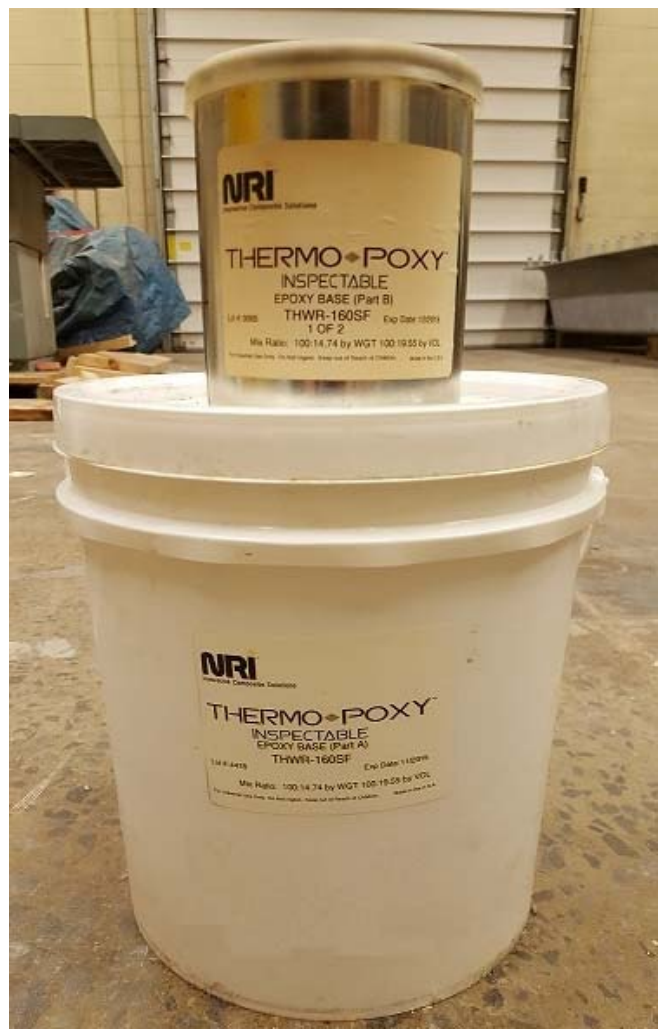

Figure 3-2 Epoxy Resin part 1 and part 2 


\subsubsection{HFRP Pre-preg}

HFRP pre-preg is a bi-axial, hybrid fabric of carbon and glass fiber which is a pre-impregnated system with polyurethane resin (Figure 3-3). Typical fiber weight fraction is $40-44 \%$ of carbon fiber to $16-20 \%$ of glass fiber. The mechanical properties are presented in Table 3-3. Mix ratio of part A to part B is 100: 54.4 by weight.

Table 3-3 Mechanical Properties of HFRP Pre-preg

Note: Values from Manufacturer

\begin{tabular}{|c|c|c|c|}
\hline \multicolumn{2}{|c|}{ Property } & $\begin{array}{c}\text { Circumferential } \\
\text { Direction }\end{array}$ & $\begin{array}{c}\text { Axial } \\
\text { Direction }\end{array}$ \\
\hline \multicolumn{2}{|c|}{ Tensile Modulus (psi) } & $8 \times 10^{6}$ & $2 \times 10^{6}$ \\
\hline \multicolumn{2}{|c|}{ Laminate Thickness (in.) } & \multicolumn{2}{|c|}{0.018} \\
\hline \multirow{2}{*}{$\begin{array}{l}\text { Primer } \\
\text { Viscosity }\end{array}$} & Part A & \multicolumn{2}{|c|}{$19,599 \mathrm{cP}$} \\
\hline & Part B & \multicolumn{2}{|c|}{$4004 \mathrm{cP}$} \\
\hline
\end{tabular}

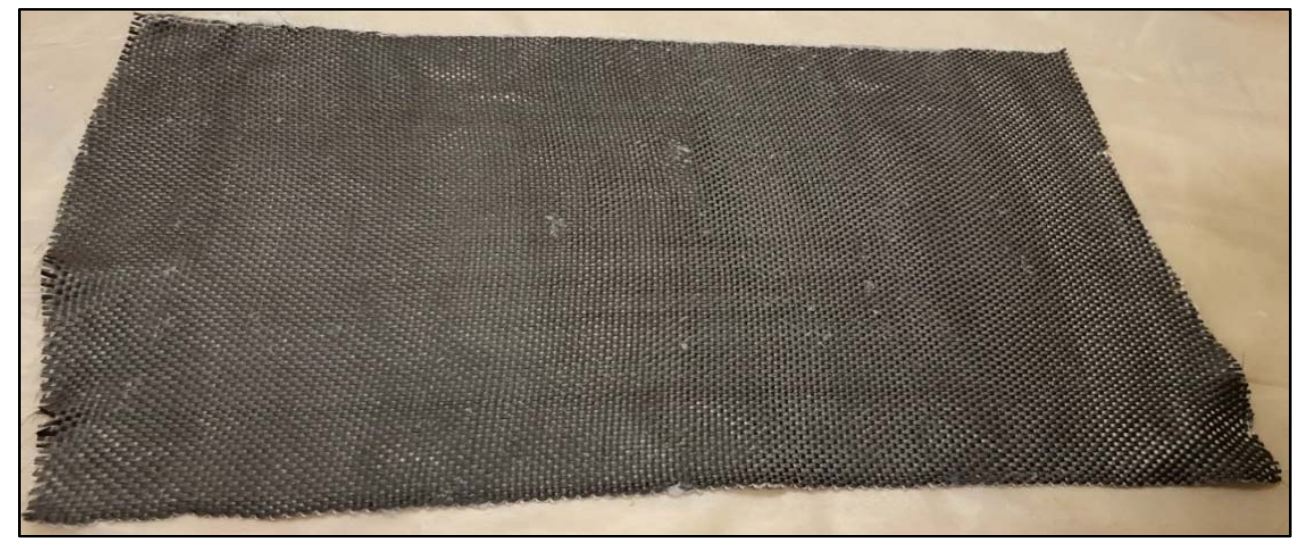

Figure 3-3 Carbon and Glass Hybrid Fabric

\subsection{Summary}

The types of materials used in this research work and their properties are presented in this chapter. 


\section{TENSION TESTS ON FRP MATERIALS}

\subsection{Introduction}

This chapter consists of the test results of the tension test on FRP coupons and direct tension test (pull-off method) on FRP overlay wrapped onto concrete substrate. The results are presented in tables and graphs. FRP coupons were tested to calculate the mechanical properties of the material and pull-off tests were conducted to evaluate the bond strength between FRP overlay and the concrete substrate. The tension results are discussed and analyzed in this chapter.

\subsection{Test Description}

\subsubsection{Tension Tests on FRP Coupons}

Tension tests on CFRP-epoxy and HFRP Pre-preg coupons were conducted on Instron-8501 as per ASTM D3039/D3039M-00 to obtain the material properties such as tensile strength and stiffness. FRP coupons of size $12 " \mathrm{x} 3 / 4$ " and $12 " \mathrm{x} 1$ " are cut from laminates that are made in 1,2 , and 3 numbers of FRP layers. Five samples were taken for each category and a total of 60 number of coupons were tested. The average load, tensile stress and stiffness are tabulated in Section 4.6.

\subsubsection{Direct Tension Test (Pull-off Method)}

Direct tension tests were conducted as per ASTM C1583/C1583M-13 to calculate the nominal tensile stress at failure by recording the failure load and failure mode. This method helps in evaluating the bond strength between the repair material overlay and concrete surface substrate, which is an important factor to be considered for the efficiency of the repair material that ensures the load transfer mechanism from deteriorated structural components to the repair material to increase the service period of the structure. Three samples were considered for each category and 
a total of 24 core samples were tested. The average load, bond stress and failure mode are tabulated in Section 4.6.

\subsection{Equipment and Test Set-up}

\subsubsection{Instron Machine}

The Instron-8501 machine (Figure 4-1) with a capacity of 22 kip, was used to test the FRP coupons to determine tensile strength and stiffness.

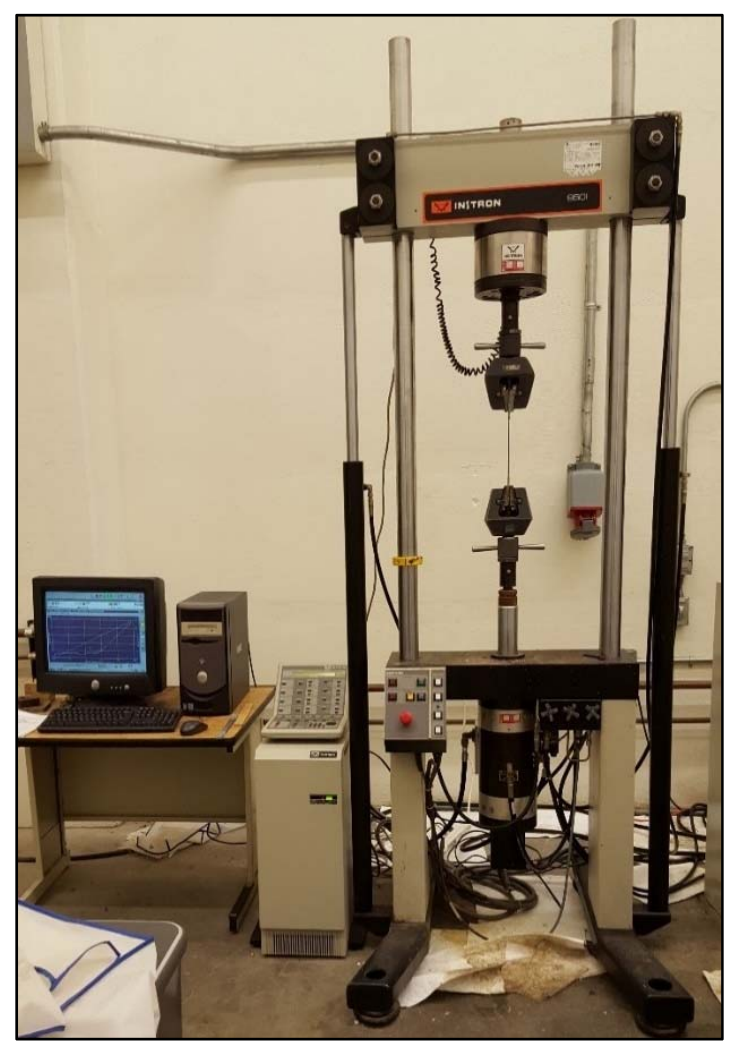

Figure 4-1 Instron-8501 Machine

\subsubsection{Proceq-DYNA Z16 Pull-off Tester}

The Proceq-DYNA Z16 pull-off tester (Figure 4-2) was used for conducting direct tensile tests on the FRP samples to evaluate the bond strength between FRP overlay and concrete substrate. 

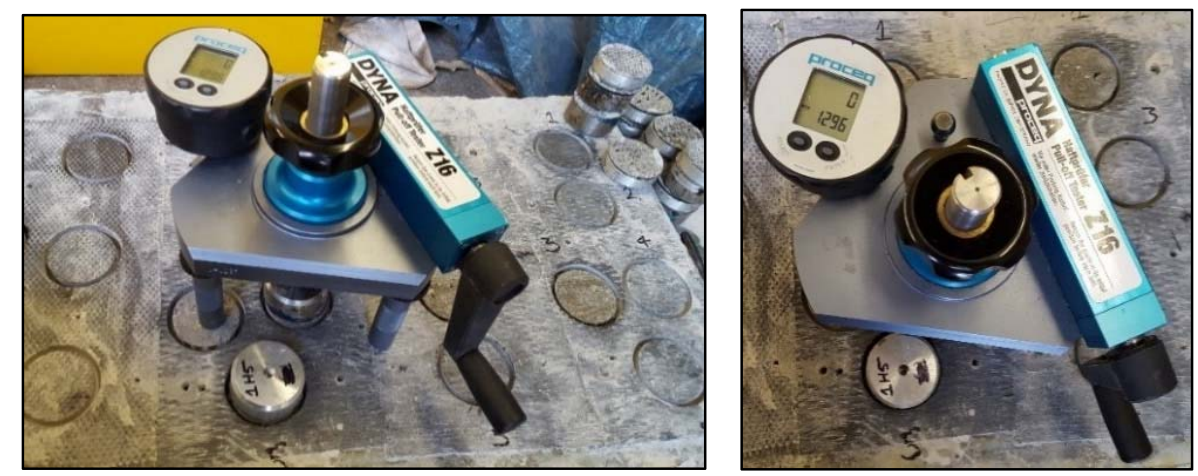

Figure 4-2 Proceq-DYNA Z16 Pull-off Tester

\subsection{Strain Gages and Data Acquisition}

To record and collect the test data, data acquisition system 8000 was used. It has strain gage cards and high level cards with a capacity of eight channels. For coupon tensile testing, strain gage cards are used to record data from strain gages those installed on the FRP coupon specimens. STRAIN SMART software (Figure 4-3(b)) was used to interpret the acquired data by exporting the recorded readings in to excel files. Strain gages used for data recording were manufactured by Vishay Micro-measurements with a resistance of $350 \mathrm{ohm}$ (Figure 4-3(a)). M200-bond from Vishay Micro-measurements was used to attach them on to the surface of CFRP-epoxy coupons and AE10-bond for Hybrid prepreg coupons. Load data was collected by the inbuilt load cell of Instron8501 machine and readings are exported to excel through FT-Console and Wave Maker softwares.

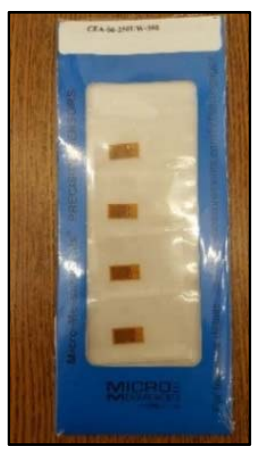

(a)

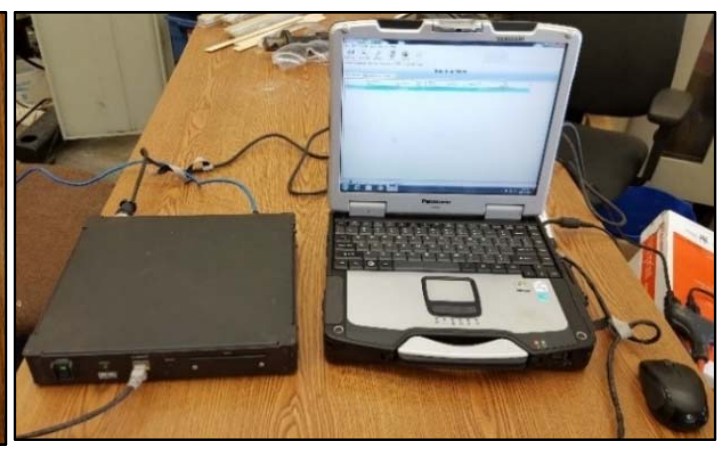

(b)

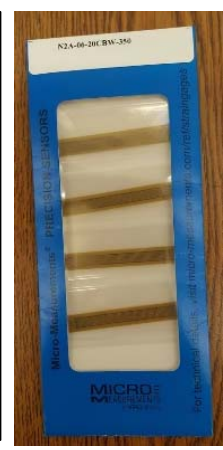

(c)

Figure 4-3 (a) 350 Ohm Strain Gages (b) Data Acquisition System-8000 (c) 350 Ohm long Strain Gages 


\subsection{Specimen Preparation}

\subsubsection{FRP Coupon Specimens:}

CFRP-epoxy and Hybrid prepreg strip specimens of 3/4" in width and 12" in length were prepared to determine the tensile properties of fiber. The thickness of the CFRP-epoxy fabric was 0.034" and of the Hybrid prepreg fabric was 0.018 ". Steps for preparation of FRP coupon specimens are as follows:

\subsubsection{CFRP-epoxy Coupons}

i. CFRP fabric was cut into a size of 13"x12" (13" length along fiber direction).

ii. $\quad$ Epoxy resin was mixed as given in section 3.4.2.

iii. Saturate the CFRP sheet with the mixed resin on both sides of the sheet.

iv. Make laminates by placing sheets one over the other in the required number of layers $(1,2$, and 3 layers).

v. Place the laminates in between the heavy iron plates by applying releasing agent on to the surfaces of these plates so that CFRP sheets do not stick on to them.

vi. Leave them aside for 24 hours of curing time.

vii. After curing, cut them into the strips of size 12 " $\mathrm{x} 3 / 4$ " for all specimens and additional strips of 12 " x 1" for 3 layer specimen alone.

viii. Attach perforated steel tabs of size 3" length on both the end of the coupons leaving a gage length of 6" at center.

ix. Attach strain gages at mid height of the coupon along the axial direction (Figure 4-4). 


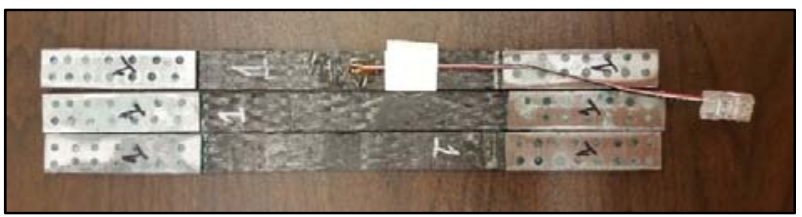

Figure 4-4 CFRP-epoxy coupons

\subsubsection{Hybrid Pre-preg Coupons}

i. Hybrid pre-preg fabric was cut into a sheet of size 13"x12" (13" length along fiber direction).

ii. Make laminates by placing sheets one over the other in the required number of layers $(1,2$, and 3 layers).

iii. Place the laminates in between the heavy iron plates by applying releasing agent on to the surfaces of these plates so that HFRP sheets do not stick on to them.

iv. Leave them aside for 5 days of curing time.

v. After the laminate hardens, cut them into the strips of size 12 " $\mathrm{x} 3 / 4$ " for all specimens and additional strips of $12 " \mathrm{x} 1$ " for 2 layer specimen alone. Coupons were made both in carbon direction and glass direction.

vi. Attach perforated steel tabs of size 3" length on both the end of the coupons leaving a gage length of 6" at center to avoid the failure in grips.

vii. Attach strain gages at mid height of the coupon along the axial and transverse directions (Figure 4-5).

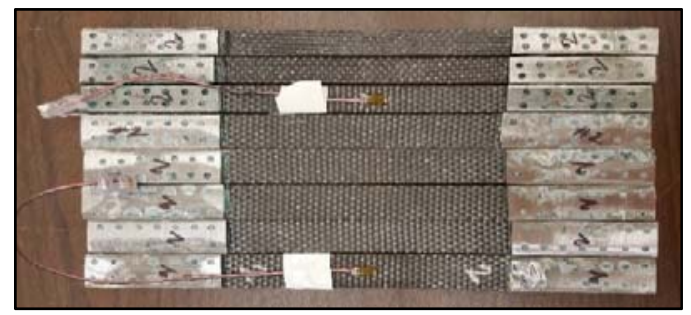

Figure 4-5 Hybrid pre-preg coupons 


\subsubsection{Pull-off Specimens:}

Steps for preparation of FRP pull-off specimens are as follows:

i. Wire brush concrete surface and wipe it clean. Attach FRP fabric of dimension 6" x 12" on to the flat surface of concrete slab of $5.5 \mathrm{ksi}$ as shown in Figure 4-6.

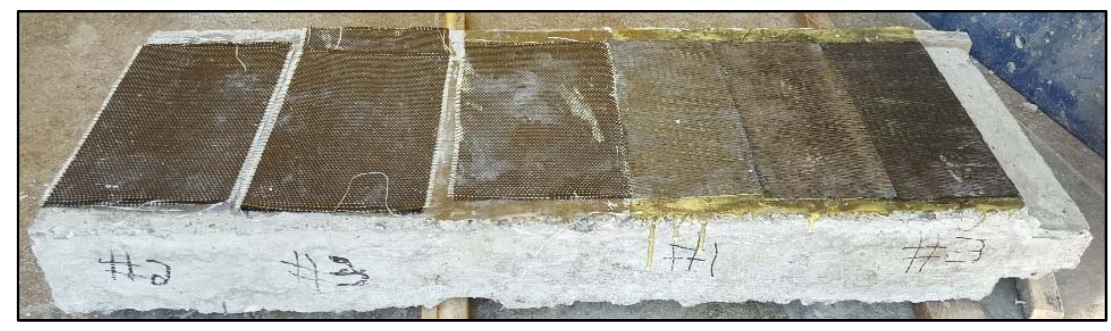

Figure 4-6 FRP overlay on concrete substrate (1,2, and 3 layers)

ii. After curing the wrap, drill a circular notch of dimension 2" diameter using the coring equipment with a diamond drill bit to a depth(minimum) of 0.5 " below the interface of concrete-FRP overlay (Figure 4-7).

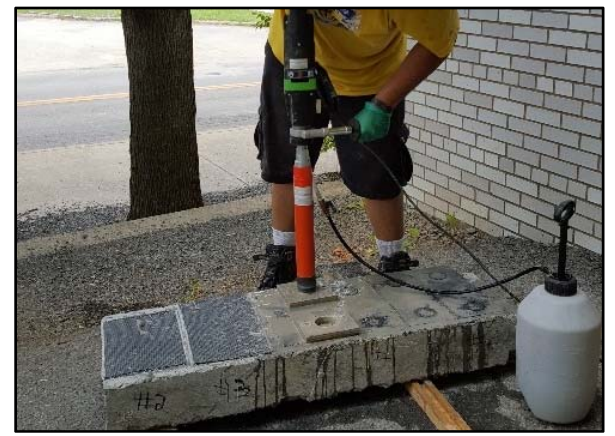

Figure 4-7 Drilling 2" diameter notches on the test specimen

iii.Clean the test specimen with water and let the surface dry with no standing water (Figure 4-8).

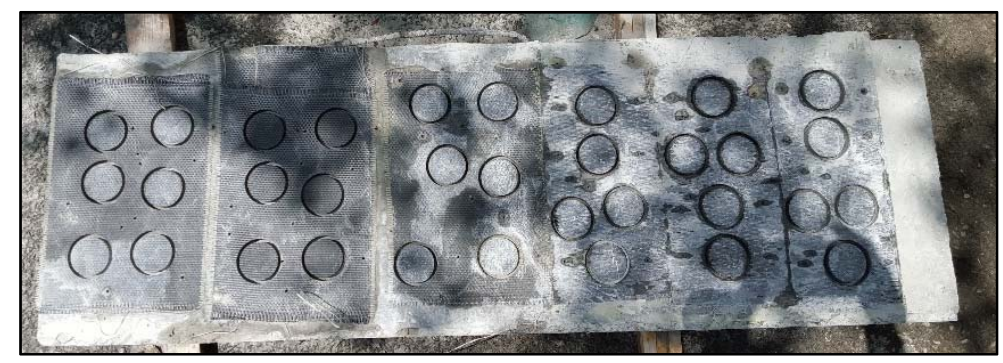

Figure 4-8 Test specimen with 2" diameter notches 
iv. Smoothen the surface of notched FRP with a sand paper and wipe with conditioner to ensure proper bonding with epoxy.

v. Attach 2" aluminum dollies to the prepared surface of FRP notch using quick setting epoxy with a strength of $3.2 \mathrm{ksi}$ ensuring the centering of the dolly with the FRP notch. Allow it to cure (Fig 4.9).

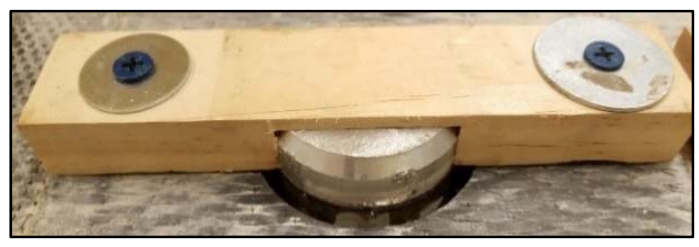

Figure 4-9 Attaching Aluminum dolly on the surface of FRP notch

\subsection{Test Procedure}

\subsubsection{FRP Coupon Specimens:}

Prepared FRP coupons as given in section 4.4 are inserted in between the fixtures installed in the Instron-8501 machine and load applied on the specimen at a low load rate in a strain controlled mode until failure as per ASTM D3039 (Fig 4.10). Load data and strain data readings were collected by the data acquisition set up (Fig 4.3).

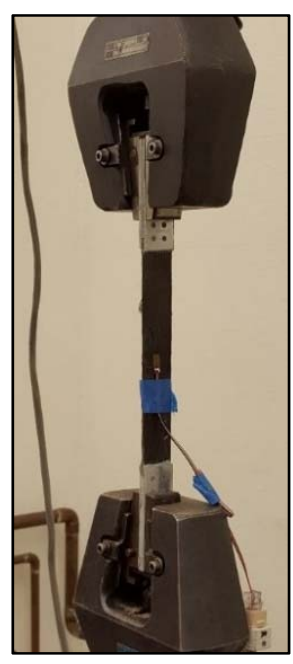

(a)

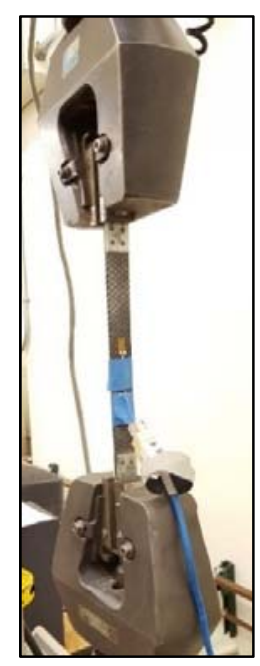

(b)

Figure 4-10 (a) Testing of CFRP Coupon (b) Testing of HFRP Pre-preg Coupon 


\subsubsection{Pull-off Tests:}

Proceq-DYNA Z16 pull-off tester was used for conducting pull-off tests on the prepared FRP notch specimens (Fig 4.11(a)). Coupling device is used to attach the device to the aluminum dolly which was fixed on to the FRP notch as described in section 4.4.2. Tensile load is applied at a constant rate until the failure of the specimen and the failure load and failure mode (Fig 4.11(b)) are recorded.

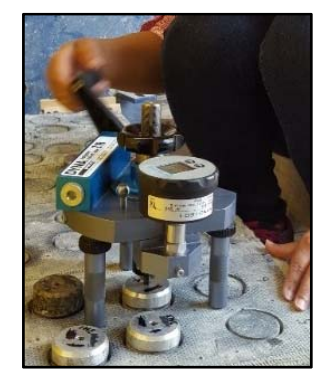

(a)

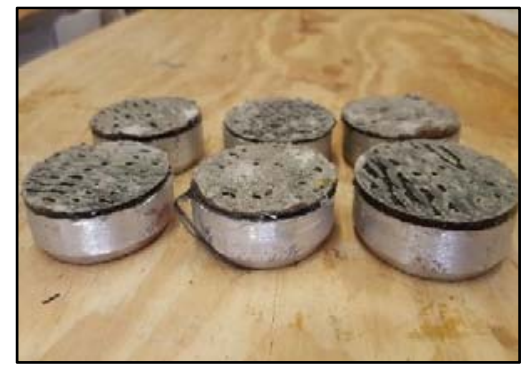

(b)

Figure 4-11 (a) Pull-off testing (b) Samples of failed specimens

\subsection{Test Results and Analysis}

\subsubsection{Tension Tests on CFRP-Epoxy Coupons}

Five specimens were tested in tension in each category of 1, 2, and 3 number of layers (Fig 4.12) and those specimens with inconsistent failure modes were not considered in the average values. The test results are tabulated in Table 4-1. Test results were plotted as stress vs strain curves and stiffness was calculated. A typical stress-strain curve for single layer CFRP-epoxy coupon is shown in Fig 4.13.

* Table 4-1 shows that one, two and three layered CFRP-epoxy coupons failed at an average load of $3.12,5.58$, and 7.79 kip with a standard deviation of $0.45,0.74$, and 0.98 respectively. And obtained an average stress of $132 \mathrm{ksi}$.

Average load per unit width per layer is $4.16 \mathrm{kip}, 3.72 \mathrm{kip}$ and $3.37 \mathrm{kip}$ for one, two and three layers respectively and for three layer 1" wide coupon it is $3.53 \mathrm{kip}$ which is almost 
in line with the value obtained from $3 / 4$ " wide coupon. Not a big difference has been observed between $3 / 4$ " or 1 " wide coupons.

* Hence, the tensile properties of CFRP coupons were calculated as given in Table 4-1.

Observed Explosive Gage Middle failure mode as per ASTM D3039.

Average strength values are lower than expected due to less saturation of fabric with high viscous resin.

Table 4-1 Tensile Properties of CFRP-Epoxy Coupons

\begin{tabular}{|c|c|c|c|c|c|c|}
\hline $\begin{array}{l}\text { No. of } \\
\text { Layers }\end{array}$ & $\begin{array}{l}\text { Size of } \\
\text { Coupon }\end{array}$ & $\begin{array}{l}\text { Average } \\
\text { Load } \\
\text { (kip) }\end{array}$ & $\begin{array}{l}\text { Average Load } \\
\text { per unit Width } \\
\text { (kip) }\end{array}$ & $\begin{array}{c}\text { Strain } \\
(\%)\end{array}$ & $\begin{array}{l}\text { Stiffness } \\
(\mathrm{msi})\end{array}$ & $\begin{array}{l}\text { Manufacturer } \\
\text { Stiffness } \\
(\mathrm{msi})\end{array}$ \\
\hline 1 & $12 " x 3 / 4 "$ & 3.12 & 4.16 & 0.93 & \multirow{4}{*}{14} & \multirow{4}{*}{12.38} \\
\hline 2 & $12 " x 3 / 4 "$ & 5.58 & 7.44 & 1.03 & & \\
\hline 3 & $12 " \mathrm{x} 3 / 4 "$ & 7.59 & 10.12 & 1.25 & & \\
\hline 3 & $12 " \times 1 "$ & 10.58 & 10.58 & 1.09 & & \\
\hline
\end{tabular}

Note: Manufacturer values are for CFRP fabric with epoxy resin

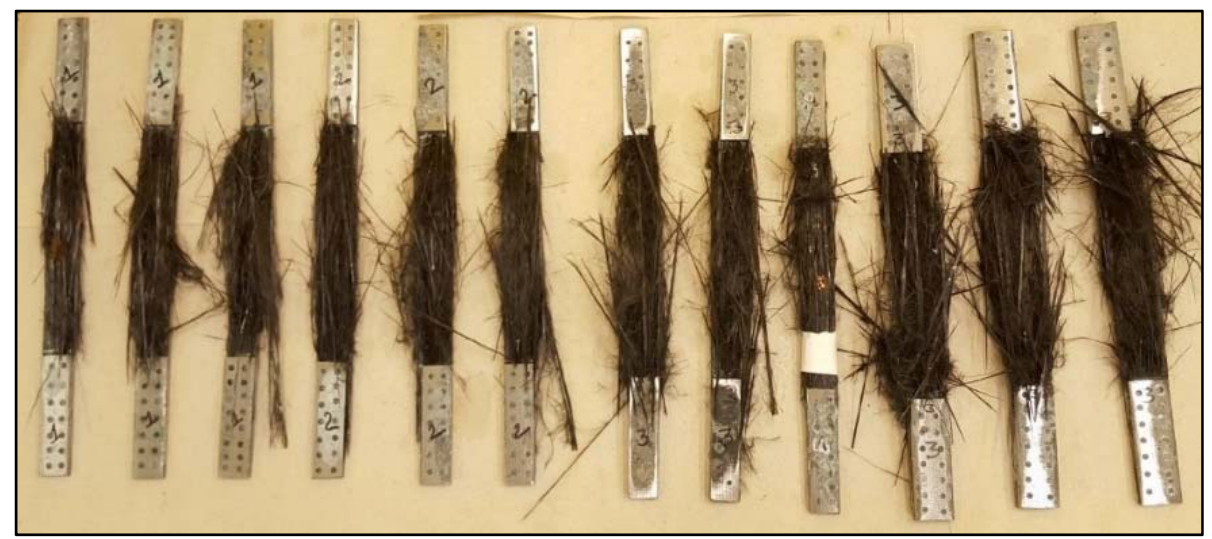

Figure 4-12 Tension Tested CFRP-Epoxy Coupons 


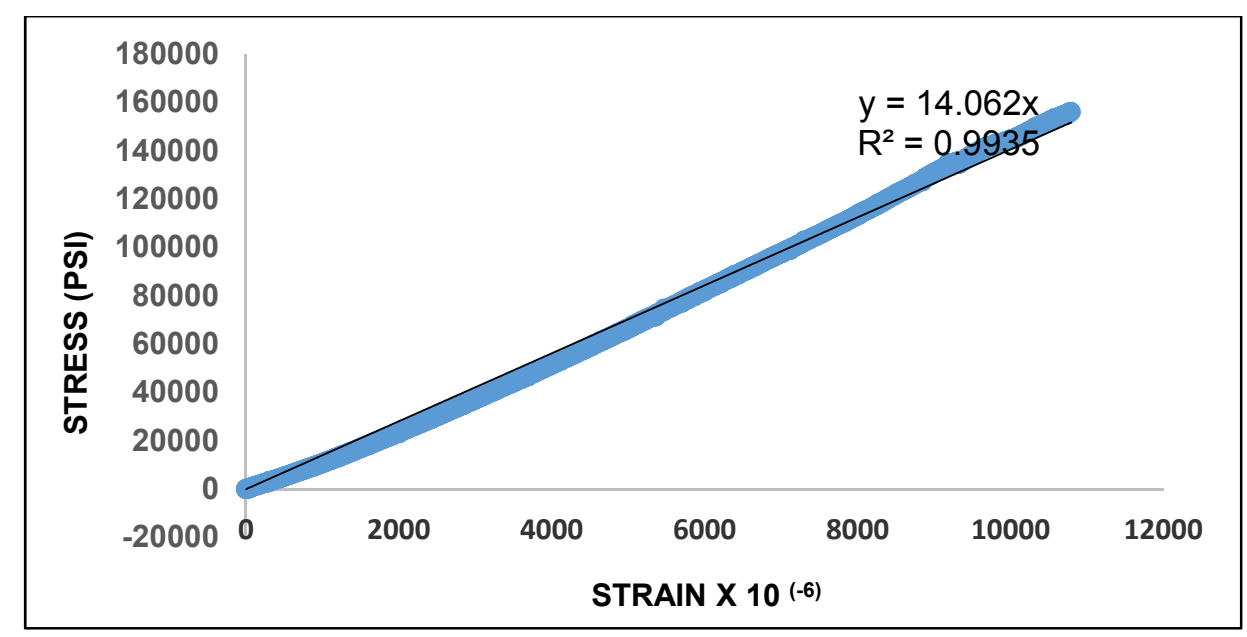

Figure 4-13 Stress-Strain Curve of Single Layer CFRP-Epoxy Coupon (Axial gage)

\subsubsection{Tension Tests on Hybrid pre-preg Coupons}

HFRP Pre-preg being the bi-directional fabric has been tested in both the directions i.e., carbon direction and glass direction separately in order to find the properties in both the directions axially. Five specimens were tested in tension in each category of 1, 2, and 3 number of layers (Fig 4.14, 4.15). The test results are tabulated in Tables 4-2 and 4-3. Test results were plotted as stress vs strain curves and stiffness was calculated. A typical stress-strain curve for three layer Hybrid prepreg coupon oriented in carbon and glass directions are shown in Fig 4.16 to 4.18.

Table 4-2 Tensile Properties of Hybrid Pre-preg Coupons in Carbon Direction

\begin{tabular}{|c|c|c|c|c|c|}
\hline No. of Layers & Size of Coupon & $\begin{array}{l}\text { Average Load } \\
\quad \text { (kip) }\end{array}$ & $\begin{array}{c}\text { Average Load } \\
\text { per unit Width } \\
\text { (kip) }\end{array}$ & $\begin{array}{l}\text { Strain } \\
(\%)\end{array}$ & $\begin{array}{l}\text { Stiffness } \\
\text { (msi) }\end{array}$ \\
\hline 1 & $12 " x 3 / 4 "$ & 1.97 & 2.64 & 1.57 & \multirow{4}{*}{8.87} \\
\hline 2 & $12 " x 3 / 4 "$ & 4.05 & 5.40 & 1.42 & \\
\hline 2 & $12 " \times 1 "$ & 5.54 & 5.54 & 1.48 & \\
\hline 3 & $12 " x 3 / 4 "$ & 5.02 & 6.69 & 1.29 & \\
\hline
\end{tabular}

Table 4-2 shows that one, two and three layered Hybrid prepreg coupons failed at an average load of $1.97,4.05$, and 5.02 kip with a standard deviation of $0.12,0.53$, and 0.30 respectively. And obtained an average stress of 144 ksi. 
Average load per unit width per layer is 2.64 kip, 2.7 kip and 2.23 kip for one, two and three layers respectively and for two layer 1" wide coupon it is 2.77 kip which is almost in line with the value obtained from $3 / 4$ " wide coupon. Not a big difference has been observed between $3 / 4$ " or 1 " wide coupons.

Hence, the tensile properties of Hybrid pre-preg coupons oriented in carbon direction were calculated as given in Table 4-2.

Table 4-3 Tensile Properties of Hybrid Pre-preg Coupons in Glass Direction

\begin{tabular}{|c|c|c|c|c|c|}
\hline No. of Layers & Size of Coupon & $\begin{array}{c}\text { Average Load } \\
\text { (kip) }\end{array}$ & $\begin{array}{c}\text { Average Load } \\
\text { per unit Width } \\
\text { (kip) }\end{array}$ & $\begin{array}{c}\text { Strain } \\
(\%)\end{array}$ & $\begin{array}{c}\text { Stiffness } \\
\text { (msi) }\end{array}$ \\
\hline 1 & $12 " \times 3 / 4 "$ & 0.34 & 0.45 & 1.4 & \multirow{2}{*}{2.06} \\
\cline { 1 - 5 } 2 & $12 " \mathrm{x} 3 / 4 "$ & 0.87 & 1.16 & 3.5 & \\
\hline 3 & $12 " \mathrm{x} 3 / 4 "$ & 1.37 & 1.83 & 3.7 & \\
\hline
\end{tabular}

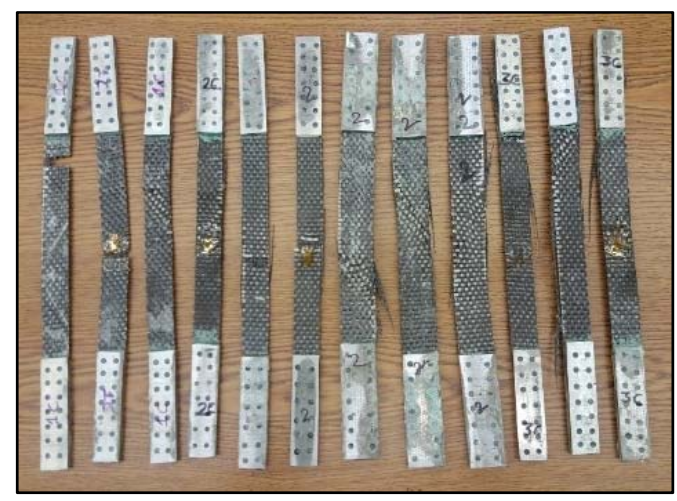

Figure 4-14 Tension Tested Hybrid pre-preg Coupons Oriented in Carbon Direction

Table 4-3 shows that one, two and three layered Hybrid pre-preg coupons failed at an average load of $0.34,0.87$, and $1.37 \mathrm{kip}$ with a standard deviation of $0.03,0.06$, and 0.14 respectively. And obtained an average stress of $30 \mathrm{ksi}$.

* Average load per unit width per layer is $0.46 \mathrm{kip}, 0.58 \mathrm{kip}$ and $0.59 \mathrm{kip}$ for one, two and three layers respectively.

Hence, the tensile properties of Hybrid pre-preg coupons oriented in glass direction were calculated as given in Table 4-3. 


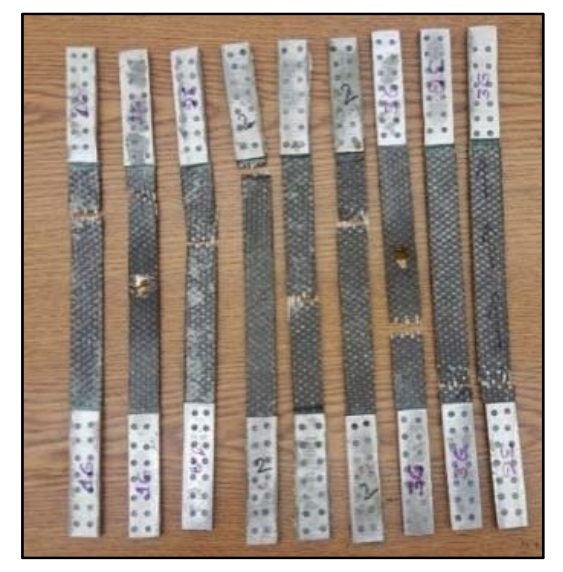

Figure 4-15 Tension Tested Hybrid pre-preg Coupons Oriented in Glass Direction

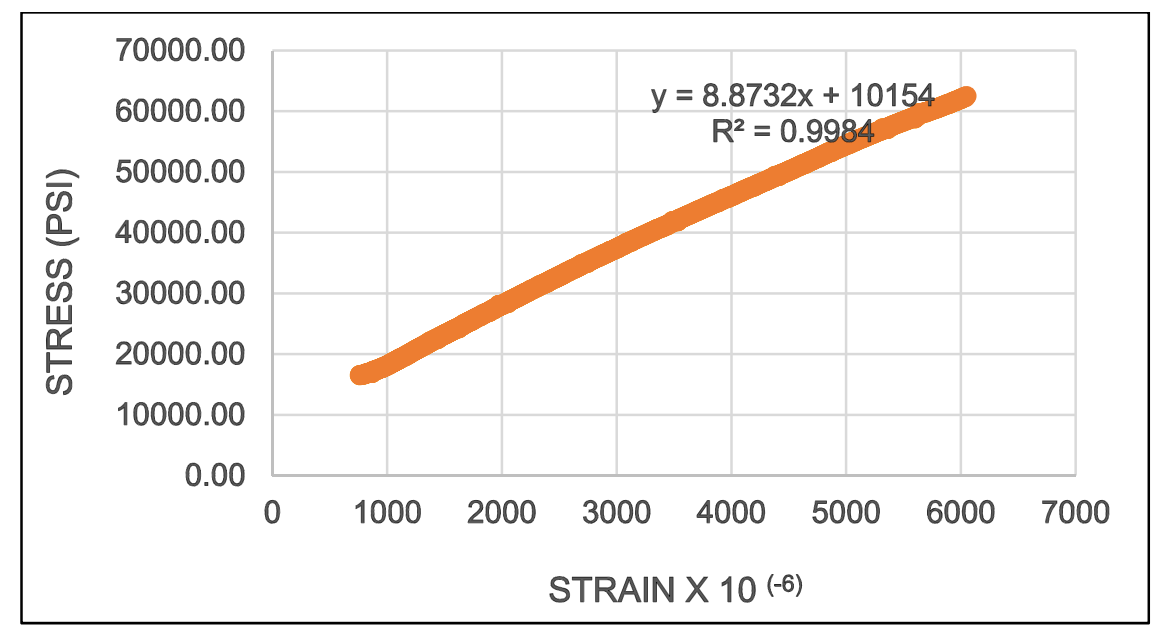

Figure 4-16 Stress-Strain Curve of Three Layer Hybrid pre-preg Coupon Oriented in Carbon Direction (Axial gage)

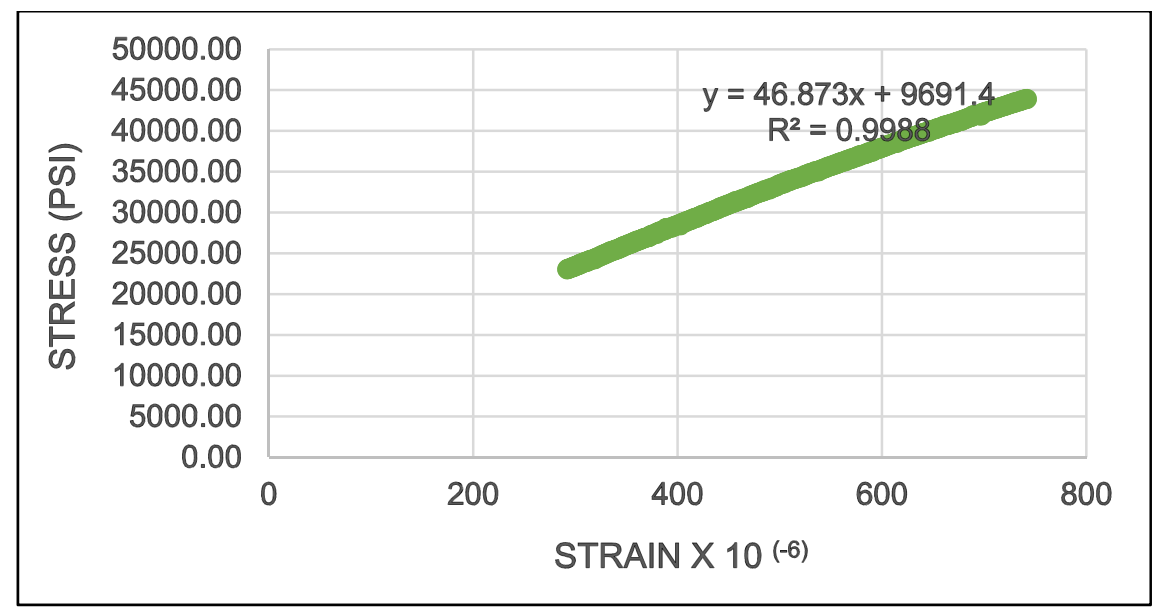

Figure 4-17 Stress-Strain Curve of Three Layer Hybrid pre-preg Coupon Oriented in Carbon Direction (Transverse gage) 


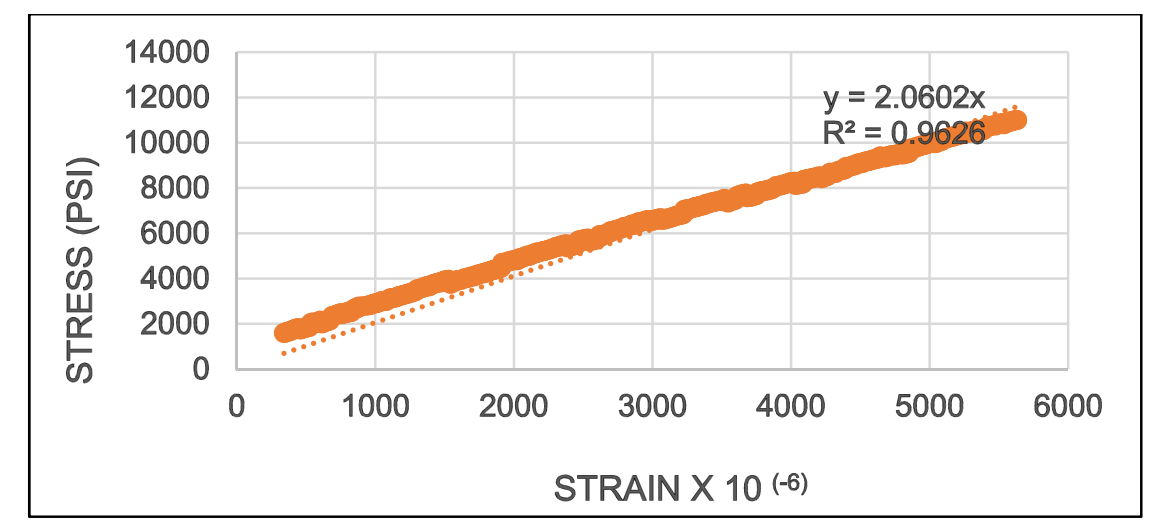

Figure 4-18 Stress-Strain Curve of Three Layer Hybrid pre-preg Coupon Oriented in Glass Direction (Axial gage)

\subsubsection{Pull-off tests}

Pull-off tests were conducted on both CFRP-epoxy and HFRP pre-preg systems with 1,2 and 3 number of layers (Fig 4.19). Three specimens were considered for each category. The average bond strength values obtained by pull-off method are tabulated in Table 4-4 and 4-5. The specimens with failure modes such as failure in substrate and failure at the interface of concrete and FRP overlay were considered for the average bond strength calculations. Other failure modes such as failure in FRP overlay and bond failure at the interface of FRP overlay and epoxy were discarded.

Table 4-4 Test Results of Pull-off Tests

\begin{tabular}{|c|c|c|c|c|}
\hline \multirow{2}{*}{ FRP System } & No. of Layers & $\begin{array}{c}\text { Avg. Tensile Load } \\
\text { (lbs) }\end{array}$ & $\begin{array}{c}\text { Avg. Bond Stress } \\
\text { (psi) }\end{array}$ & $\begin{array}{c}\text { \#Failure } \\
\text { Modes }\end{array}$ \\
\hline \multirow{3}{*}{ Hybrid prepreg } & 1 & 855 & 272 & (a) \\
\cline { 2 - 5 } & 2 & 658 & 209 & (b) \\
\cline { 2 - 5 } & 3 & 469 & 149 & (b)/(c) \\
\hline \multirow{3}{*}{ CFRP-Epoxy } & 1 & 1207 & 354 & (a) \\
\cline { 2 - 5 } & 2 & 987 & 314 & (a)/(b) \\
\cline { 2 - 5 } & 3 & 890 & 283 & (b) \\
\hline
\end{tabular}

Note: ${ }^{~}$ Failure modes as per ASTM C1583: (a) Failure in substrate; (b) Bond failure at concrete or overlay interface; (c) Failure in overlay or repair material; (d) Bond failure at epoxy/overlay interface.

Bond strength was calculated by dividing the tensile load at failure by the area of the test specimen as per ASTM C1583. With increase in number of layers, bond failures were observed at concrete 
substrate or FRP overlay interface and hence reduction in the bond strength was observed (Table 4-4) for both the varieties of FRP systems.

The obtained bond strength values are more than the minimum (200 psi) requirement for FRP wraps as given in ACI 440.2R-02 except for 3 layered HFRP pre-preg. Lower bond strength for 3 layered HFRP pre-preg is due to the primer between concrete and FRP being absorbed into undulations of additional woven hybrid fabrics.

Another specimen was tested with the surface preparation done by power wash instead of wire brush and the results were compared in Table 4-5. It shows a good improvement in the bond strength with power wash surface preparation. Resin penetration into surface pores is high with power washed surface due to increase in surface area for wetting and thus helping in increasing bond strength.

Table 4-5 Test Results of Pull-off Tests with and without Powerwash of Concrete Surface

\begin{tabular}{|c|c|c|c|c|c|c|}
\hline \multirow{2}{*}{ FRP System } & \multirow{2}{*}{$\begin{array}{l}\text { No. of } \\
\text { Layers }\end{array}$} & \multicolumn{2}{|c|}{$\begin{array}{l}\text { Avg. Tensile Load } \\
\text { (lbs.) }\end{array}$} & \multicolumn{2}{|c|}{$\begin{array}{l}\text { Avg. Bond Stress } \\
(\text { psi) }\end{array}$} & \multirow{2}{*}{$\begin{array}{c}\text { Strength } \\
\text { Increase } \\
\quad(\%)\end{array}$} \\
\hline & & $\begin{array}{c}\text { Wire } \\
\text { Brush }\end{array}$ & $\begin{array}{c}\text { Power } \\
\text { wash }\end{array}$ & $\begin{array}{l}\text { Wire } \\
\text { Brush }\end{array}$ & $\begin{array}{c}\text { Power } \\
\text { wash }\end{array}$ & \\
\hline Hybrid prepreg & 2 & 658 & 1129 & 209 & 359 & 71.6 \\
\hline CFRP-Epoxy & 2 & 987 & 1185 & 314 & 377 & 20 \\
\hline
\end{tabular}

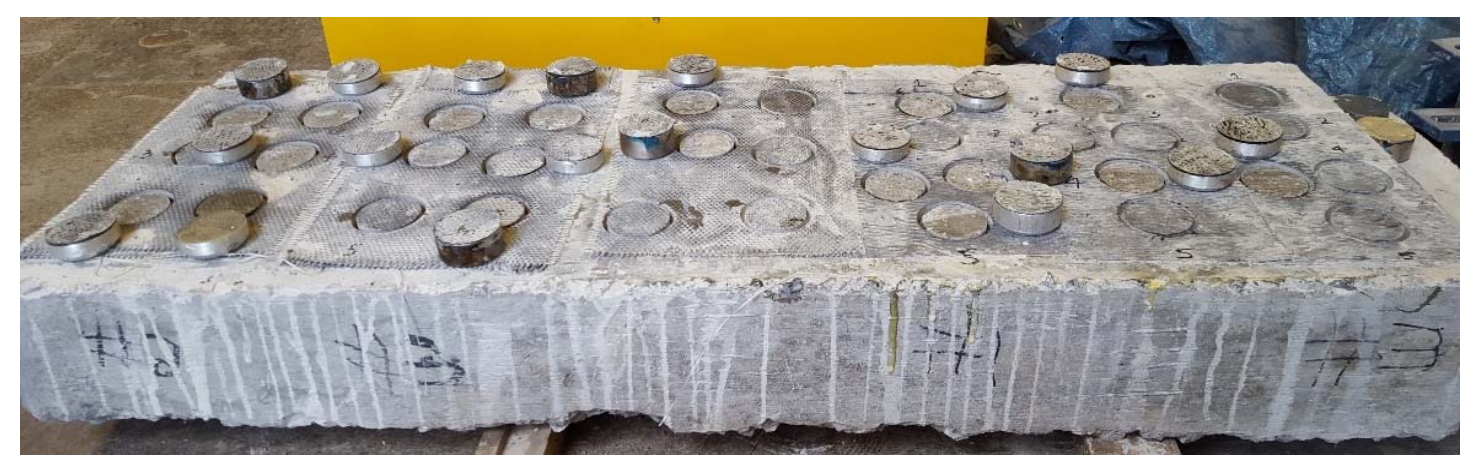

Figure 4-19 Pull-off tests on 1, 2, and 3 layers of CFRP-Epoxy and HFRP Pre-preg Overlay materials 


\subsection{Summary}

From the test results and analysis, the tensile properties and bond strength for both the FRP materials were determined.

* CFRP-epoxy has an average tensile stress of $132 \mathrm{ksi}$, rupture strain of $1.2 \%$ and stiffness of 14 msi.

* Average strength values are lower than expected due to less saturation of fabric with high viscous resin and observed Explosive Gage Middle failure mode as per ASTM D3039.

* Hybrid pre-preg in carbon direction has an average tensile stress of $144 \mathrm{ksi}$, rupture strain of $1.5 \%$ and stiffness of $8.87 \mathrm{msi}$.

- Hybrid pre-preg in glass direction has an average tensile stress of $30 \mathrm{ksi}$, rupture strain of $1.5 \%$ and stiffness of $2.06 \mathrm{msi}$.

* All the properties obtained are in line with the values provided by the manufacturer.

* No huge difference has been observed between 1" and 3/4" wide coupon properties.

* Significant improvement in bond strength was observed with efficient surface preparation such as power washing the surface before application of repair material. 


\section{CYLINDER CONFINEMENT EFFECT}

\subsection{Introduction}

This chapter consists of the test results of compression test on unconfined and confined cylinder specimens, prepared without internal reinforcement. The results are presented in tables and graphs. All specimens were wrapped manually with FRP and there was no pretension in the wrap. Comparison of load, strain, stiffness, etc. between unconfined and confined specimens has been discussed in this section. The results of different tests conducted to verify various parameters of confinement are discussed and analyzed.

\subsection{Compression Tests on Concrete Cylinders}

Concrete cylinders of size 6" x 12" from batch 1 and batch 2 as described in section 3.2 were subjected to axial compression in universal testing machine to determine the confinement effect due to CFRP-epoxy and HFRP pre-preg wrapping patterns. Cylinders are prototype models of columns. Strength, strain and stiffness presents the effect of confinement. To investigate the strengthening effect with increase in number of layers, cylinders were wrapped with 1,2, and 3 number of FRP layers. Strengthening of concrete cylinders is classified into two categories. They are Full cylinders and Split-Bonded cylinders which are further subdivided as given below:

i.Full Cylinders (Plain Cylinders)

a. Non-Wrapped cylinders

b. Wrapped cylinders

ii.Split-Bonded Cylinders

a. Non-Wrapped cylinders

b. Wrapped cylinders 


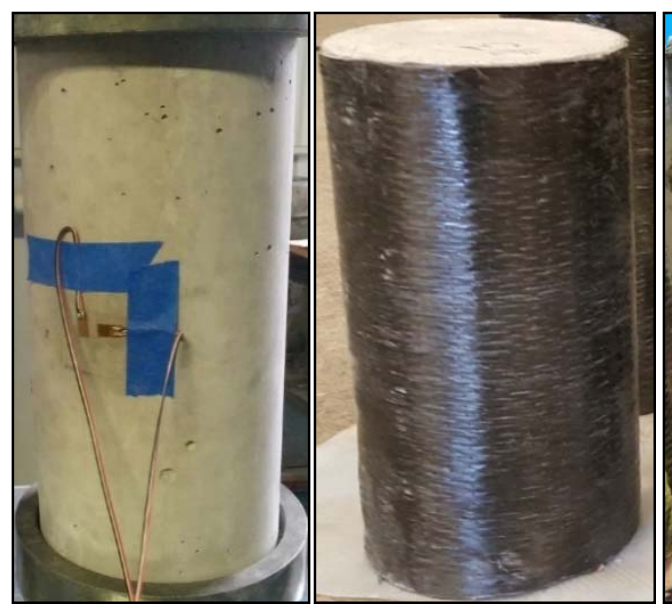

(a) (b)

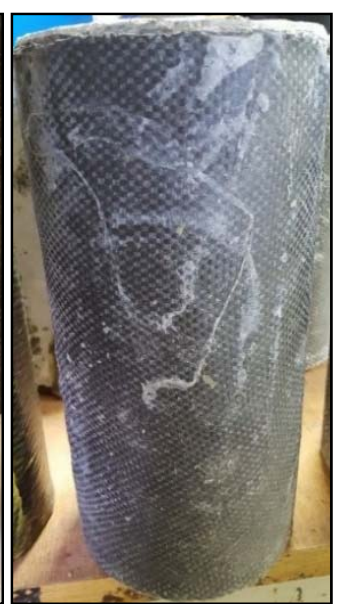

(c)

Figure 5-1 Full Cylinders: (a) Non-Wrapped Cylinders (b) CFRP Wrapped Cylinders (c) HFRP pre-preg Wrapped Cylinders

* Full Cylinders (Fig 5.1) are 6" x 12" concrete cylinders whereas Split-Bonded cylinders (Fig 5.2) are 6" x 12" cylinders formed by bonding two halves of split-cylinders using concrete adhesive to form a full cylinder.

* Split cylinders were obtained either by using separators vertically along height of the cylinder while casting or by cutting full cylinder into two halves using saw-cut. Both techniques were used and strength values found to be similar.

Non-Wrapped Full cylinders (Fig 5.1(a)) and Non-Wrapped Split-Bonded cylinders (Fig 5.2(a)) are the plain cylinders under each category tested to obtain base value for reference purpose.

Wrapped cylinders (Fig 5.1(b), 5.1(c), 5.2(b), 5.2(c)) are the cylinders wrapped with FRP wraps in 1, 2, and 3 number of layers to determine the effectiveness of the confinement and wrap efficiency. 


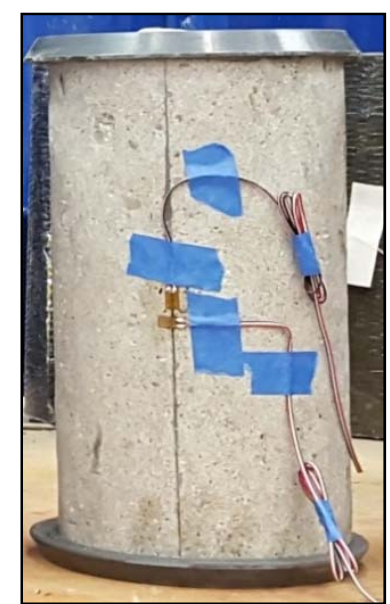

(a)

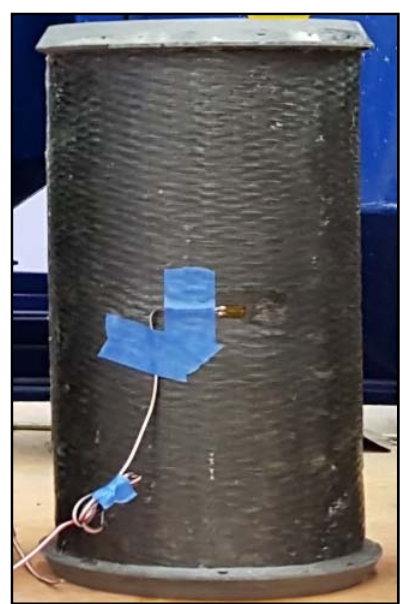

(b)

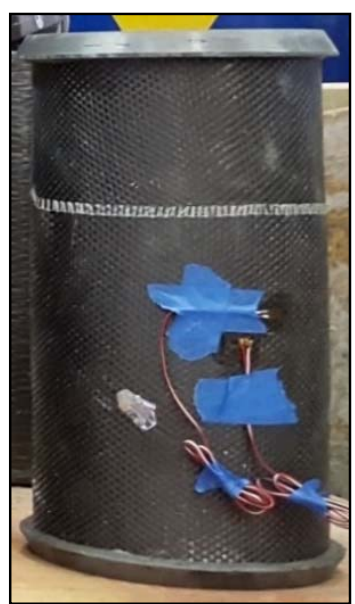

(c)

Figure 5-2 Split-Bonded Cylinders: (a) Non-Wrapped Cylinders (b) CFRP Wrapped Cylinders (c) HFRP pre-preg Wrapped Cylinders

\subsection{Equipment and Test Set-up}

\subsubsection{Universal Testing Machine (UTM)}

The universal testing machine (Fig 5.3) used for this testing has a capacity of $350 \mathrm{kip}$. Two load indicator dials shows the applied loads. One with maximum of 60 kip reading and the other with 350 kip reading. The dial with higher readings was utilized in all the tests. Load was applied by using the hydraulic jack and the speed was adjusted such that the testing takes place for at least 3 minutes to acquire considerable amount of data for analysis.

Higher strength cylinders which exceeded a capacity of 330 kip were tested at Marshall Engineering lab with a Universal Testing Machine of 500 kip capacity (Fig 5.4). It has a COPILOT digital indicator with 16-character LCD display and a range of 7 digits. It has a capacity of storing 500 test readings. Control knob was used to apply load using two stage hydraulic pump. Load applied at a rate of $40 \mathrm{psi} / \mathrm{sec}$. 


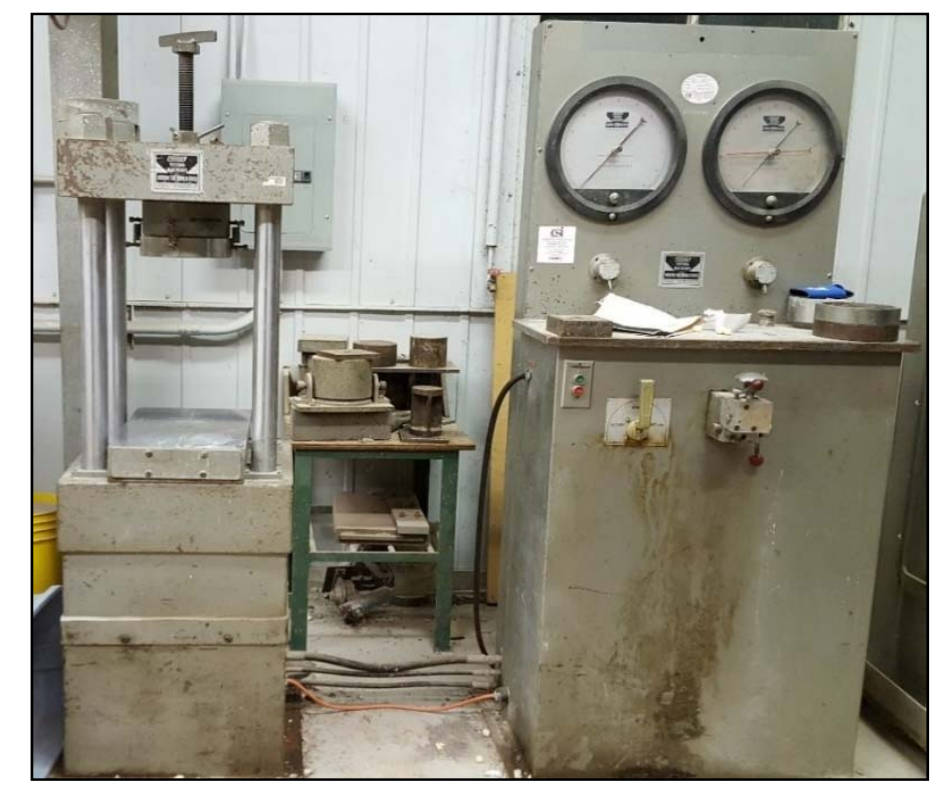

Figure 5-3 Universal Testing Machine (350 kip Capacity)

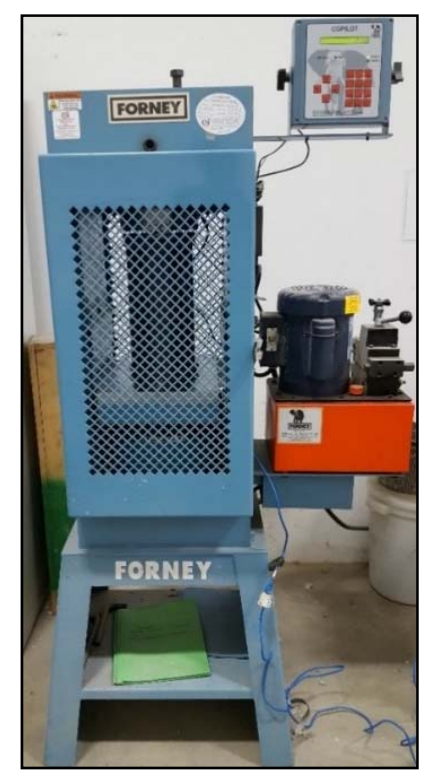

Figure 5-4 Universal Testing Machine (500 kip Capacity)

\subsubsection{Sulfur Capping Compound}

Steel caps with neoprene pads were used on both ends of the cylinders for unconfined and one layered confined cylinders whereas sulfur capping was done for two and three layered confined cylinders as they did not fit into the standard steel caps due to the increase in diameter of cylinders 
as thickness of wrap multiplied with the number of layers. Hence, according to ASTM C617/C617M-15, capping of cylinders with sulfur mortar was adopted. Sulfur capping equipment (Fig 5.5) consists of dry sulfur flakes, melting pot and ladle, capping mold with steel plate and vertical device with alignment guides, grease, rubber hammer and insulated gloves.

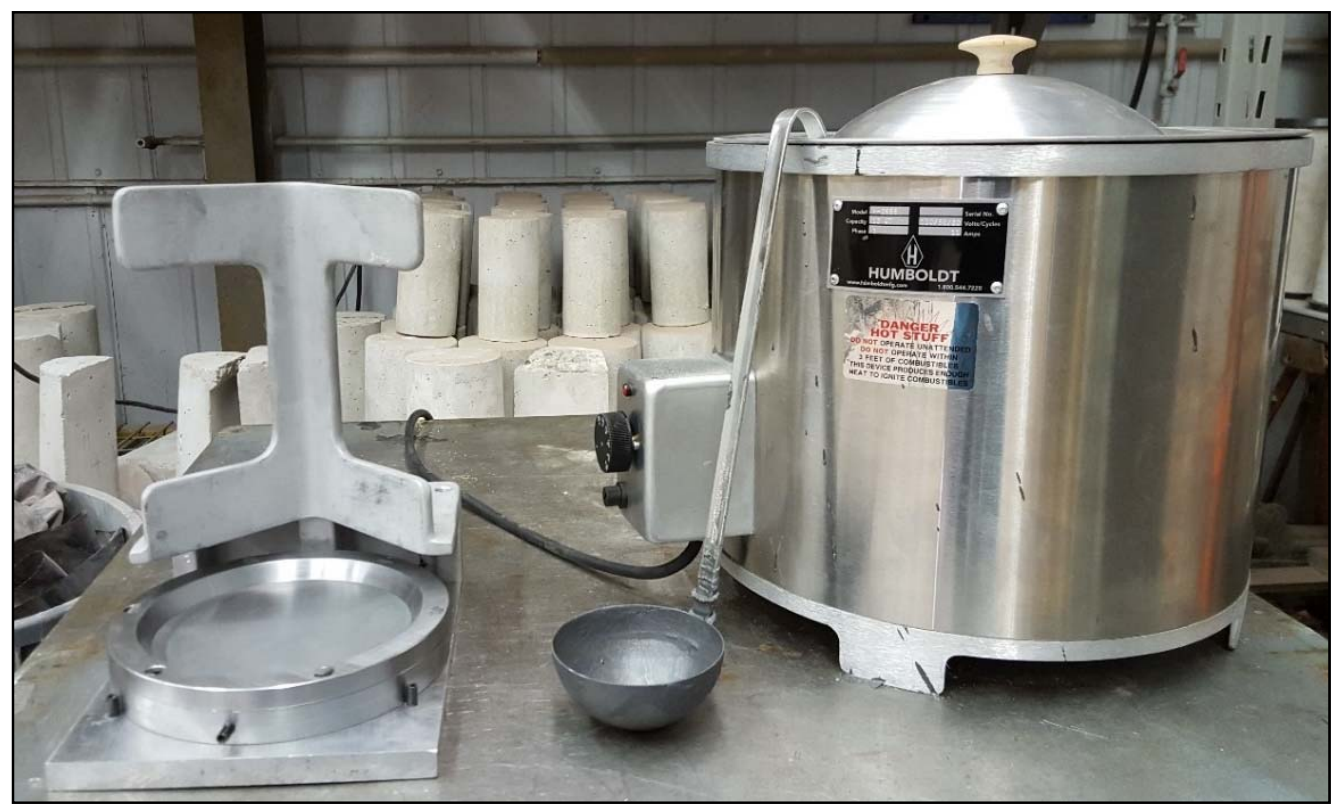

Figure 5-5 Sulfur Capping Equipment

\subsubsection{Strain Gages and Data Acquisition}

To record and collect the test data, data acquisition system 8000 was used. It has strain gage cards and high level cards with a capacity of eight channels. For coupon tensile testing, strain gage cards are used to record data from strain gages those installed at the mid height of cylinders in both axial and hoop directions. STRAIN SMART software (Fig 4.3(b)) was used to interpret the acquired data by exporting the recorded readings in to excel files. Strain gages used for data recording were manufactured by Vishay Micro-measurements with a resistance of 350ohm (Fig 4.3(a)). M200bond from Vishay Micro-measurements was used to attach them on to the surface of CFRP wrapped cylinders and AE10-bond for HFRP pre-preg wrapped cylinders. A calibrated load cell (Fig 5.6) of 300 kip capacity was connected to the strain gage card of the data acquisition system 
for obtaining continuous load data with time. Load cell was placed in the test frame of the UTM along with the test specimen to obtain the load data.

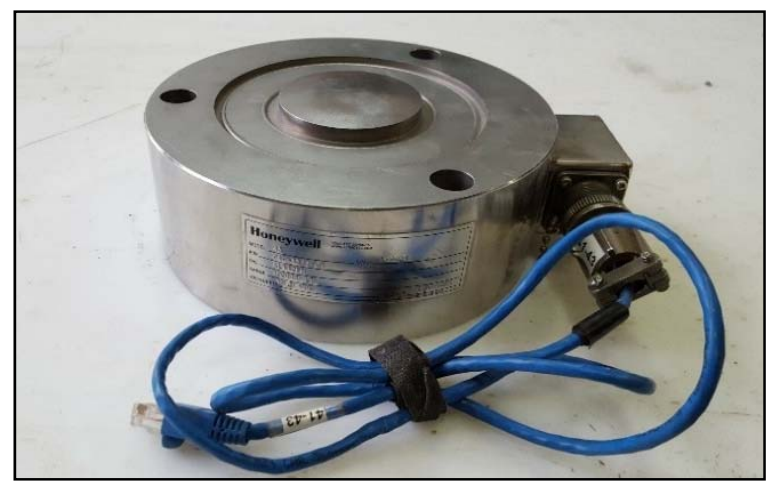

Figure 5-6 Load Cell (300 kip Capacity)

\subsection{Specimen Preparation}

\subsubsection{Casting and Curing of Concrete Cylinders}

Concrete mix was ordered from HOY Ready-mix and casting took place in two batches with two different compressive strengths. Figure 5.9 shows the lot of cured concrete cylinders. Casting and curing of the concrete cylinders were carried out as given below:

\subsubsection{Full Cylinders}

i. 6" x 12" sized plastic molds were used and were thinly coated with grease on the inner surface for facilitating ease in demolding practice.

ii. Prepared plastic molds were filled with the ready-mix concrete in three equal layers.

iii. Each layer was compacted using tamping rod giving 25 strokes each time followed by tapping on the outer sides of the molds to enable the escape of air voids through the surface.

iv. After completing the compaction procedure, the upper surface was smoothened using a finishing trowel. 
v. The filled molds are set aside for 24 hours for setting of concrete and then demolded using air hose.

vi. The demolded concrete cylinders were then shifted to a curing tank filled with water, maintained at ambient temperature and cured them for 28 days.

\subsubsection{Split Cylinders}

i. 6" x 12 " sized plastic molds were used with a plastic sheet of size 6 " x 14 " x 1/16" positioned at the center of the mold (Fig 5.7) and were thinly coated with grease on the inner surface for facilitating ease in demolding practice.

ii. Prepared plastic molds were filled with the ready-mix concrete in three equal layers on both sides of the plastic sheet maintaining it stand at the center of the mold.

iii. Each layer was compacted using tamping rod giving 25 strokes each time on both the sides followed by tapping on the outer sides of the molds to enable the escape of air voids through the surface.

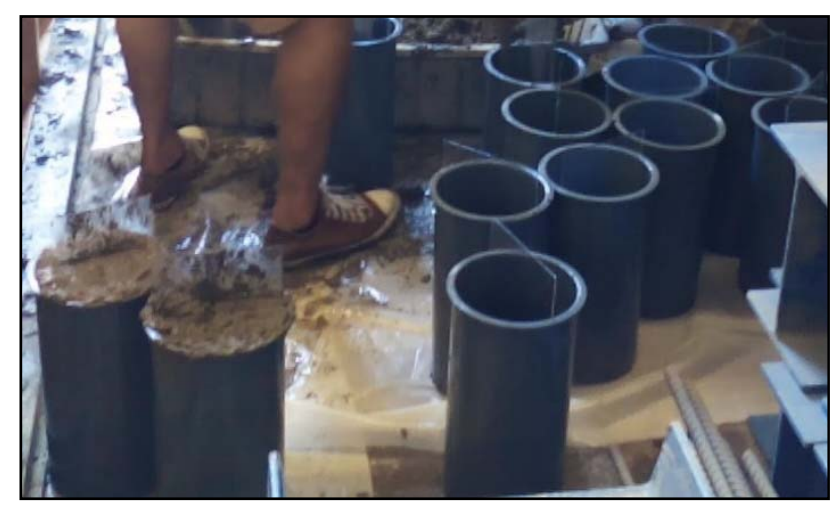

\section{Figure 5-7 Plastic Molds with Plastic Sheets for Casting Split Cylinders}

iv. After completing the compaction procedure, the upper surface was smoothened using a finishing trowel.

v. The filled molds are set aside for 24 hours for setting of concrete and then demolded. 
vi. The demolded split cylinders (Fig 5.8) were then shifted to a curing tank filled with water, maintained at ambient temperature and cured them for 28 days.

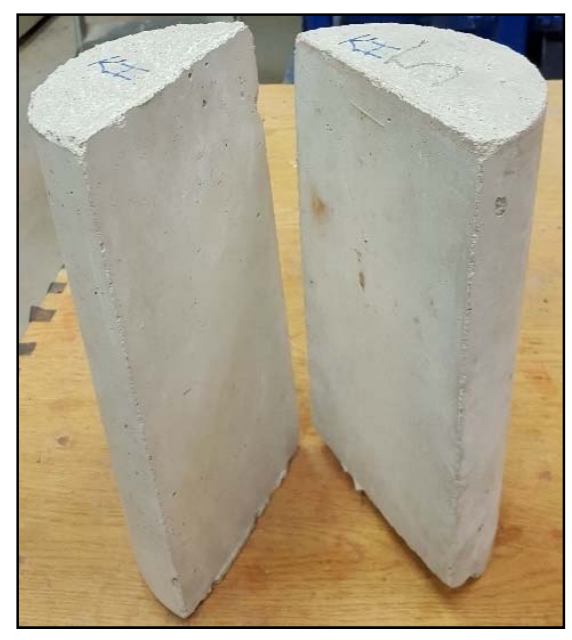

Figure 5-8 Split Cylinders after Curing for 28 Days

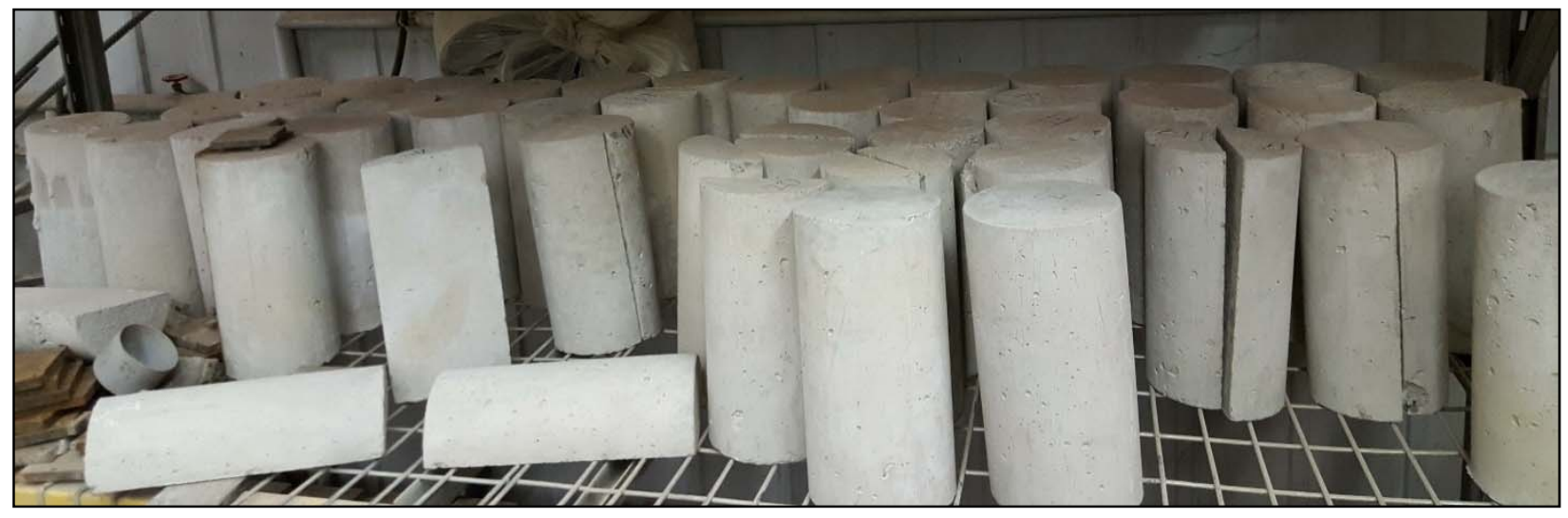

Figure 5-9 Concrete Cylinders Lot

\subsubsection{Bonding of Split Cylinders}

The split cylinders were bonded together to form a whole cylinder by using concrete adhesive Sikadur 35 Hi-Mod LV manufactured and provided by Sika Corporation U.S. Steps followed for bonding split cylinders are as follows:

i.Prepare the surface of each half of the split cylinder using wire brush and wipe off the dust with a damp cloth.

ii.Mix the concrete adhesive as per the instructions given by manufacturer. 
iii.Apply the mixed adhesive on both the prepared surfaces (Fig 5.10).

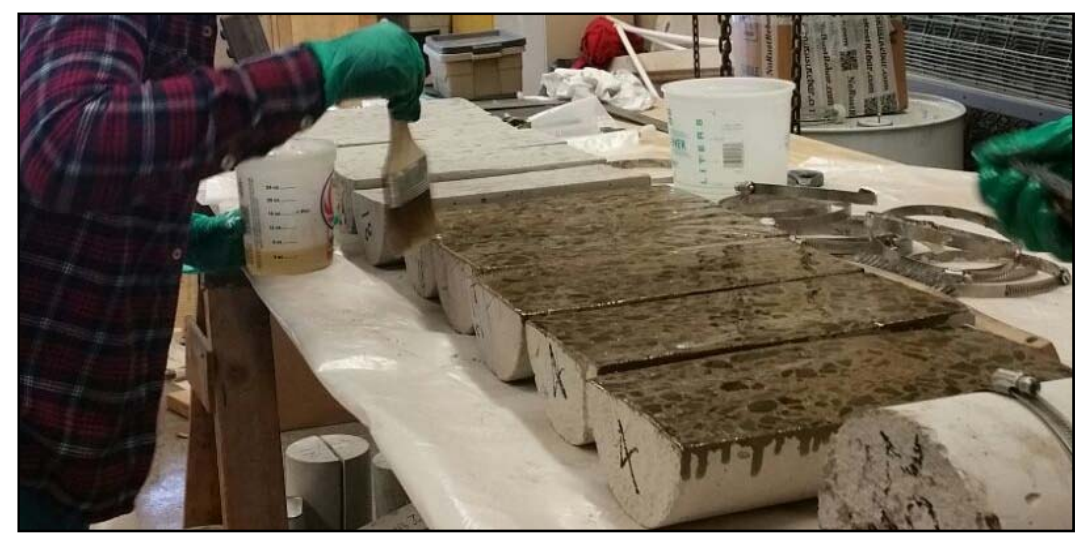

Figure 5-10 Split Cylinders with Applied Concrete Adhesive

iv.Bring the two halves together and tighten them with hose clamps at top and bottom sides of the cylinder (Fig 5.11).

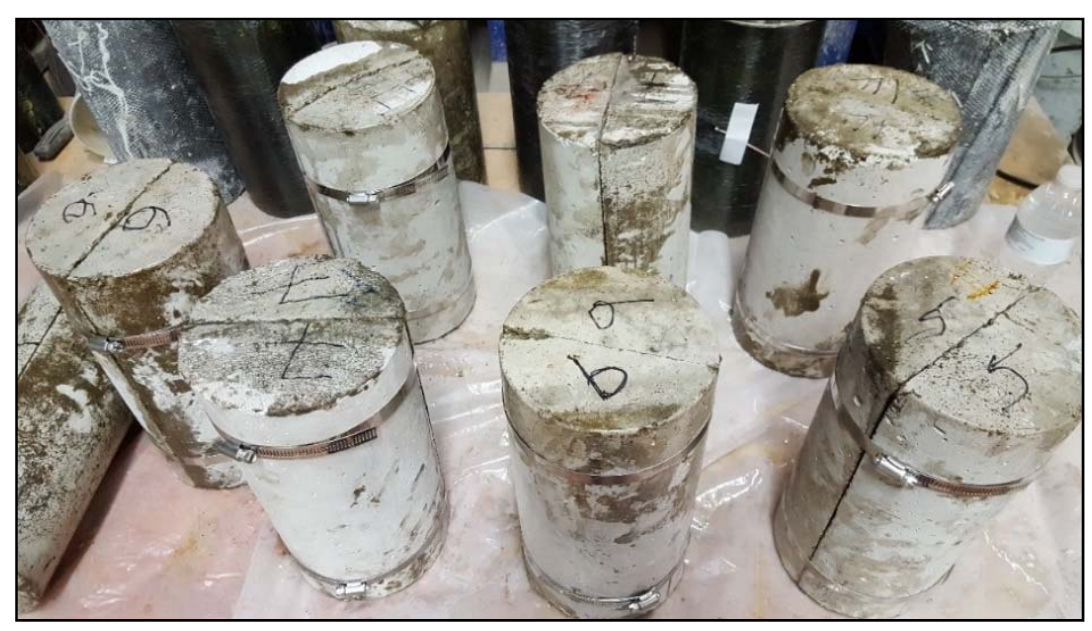

Figure 5-11 Split Cylinders with Hose Clamps

v.Set it aside for 24 hours for curing.

vi.After the setting time, remove the clamps, clean the adhesive stains on the surface of the cylinders using disc grinder and the split-bonded cylinders are ready (Fig 5.12). 


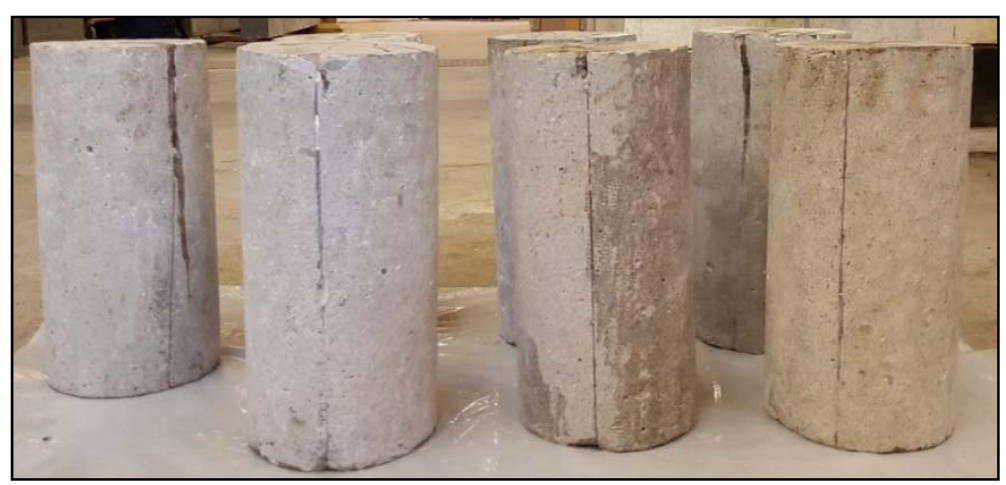

Figure 5-12 Split-Bonded Cylinders

\subsubsection{Wrapping of Cylinders with FRP}

Confinement of concrete cylinders was carried out using two types of fabrics that were provided by the manufacturer and details of the same were discussed in section 3.4. FRP fabrics were cut into lengths required for wrapping cylinders in 1, 2, and 3 layers with an overlap length of 4". The surface of cylinders were wire brushed, cleaned with damp cloth and air-hose to remove dirt particles to eliminate voids between concrete surface and FRP wrap. Wrapping procedure is as elucidated below:

\subsubsection{CFRP-Epoxy System}

i. $\quad$ Cut the fabric as per the testing scheme (Fig 5.13).

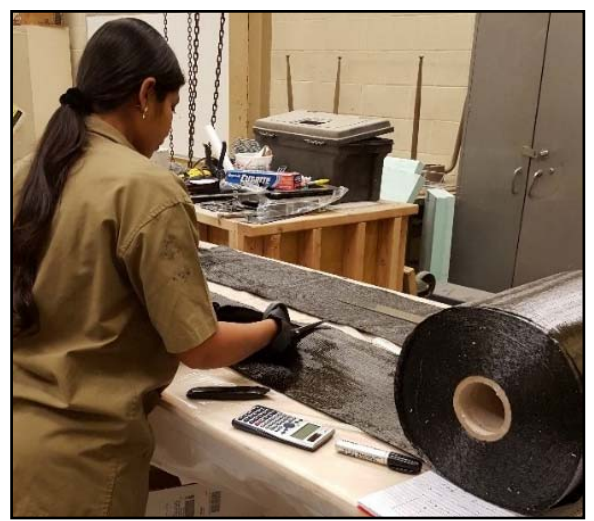

Figure 5-13 Cutting CFRP Fabric into Wraps

ii. Mix the two-part epoxy resin as described in section 3.4 (Fig 5.14). 

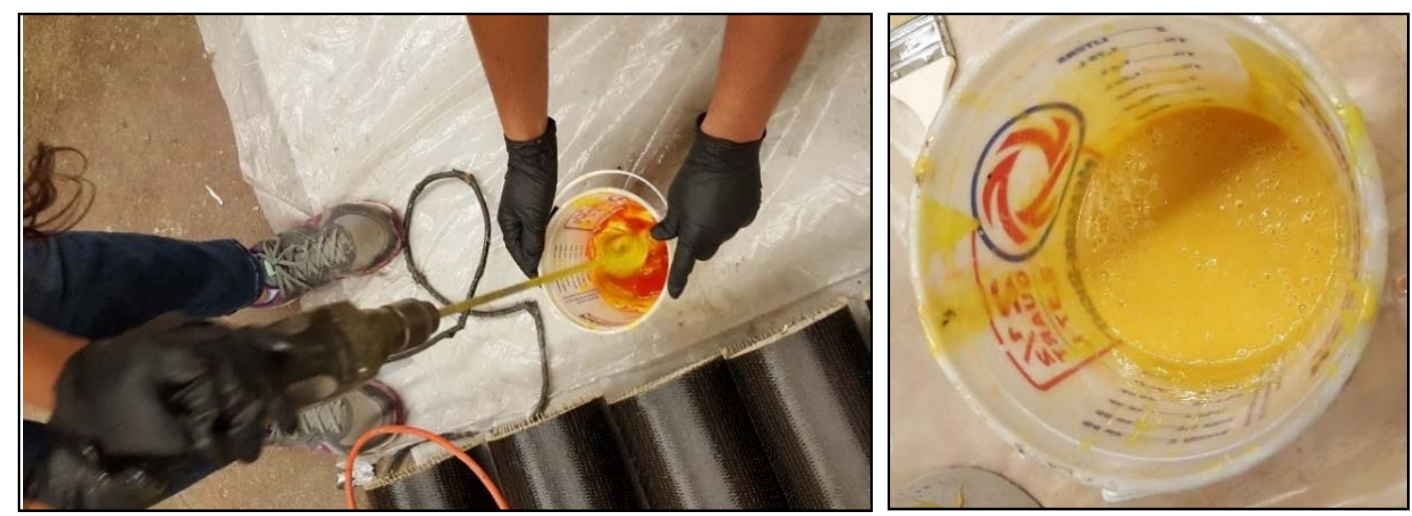

Figure 5-14 Mixing of Two-Part Epoxy (left); Mixed Resin (Right)

iii. $\quad$ CFRP fabric was saturated with the mixed epoxy resin (Fig 5.15)

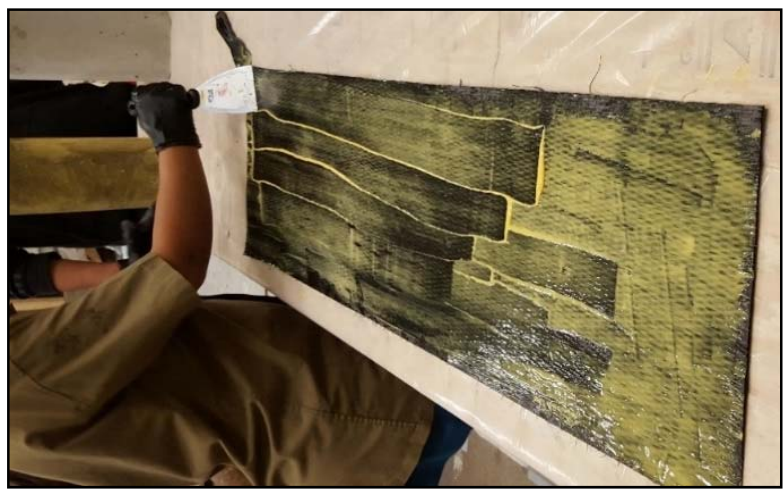

Figure 5-15 Saturating CFRP Fabric with Mixed Epoxy

iv. Apply a primer coat on the surface of concrete cylinder with the mixed resin (Fig 5.16).

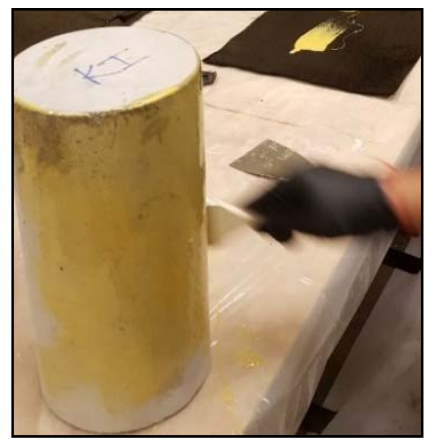

Figure 5-16 Applying Resin Primer Coat on the Surface of Cylinder

v. CFRP-epoxy system was wrapped around the concrete cylinders in 1,2, and 3 layers as per testing scheme (Fig 5.17). 
vi. Set the wrapped cylinders aside for a curing period of 24 hours in such a way that the end of the wrap faces down and cures under the weight of the cylinder (Fig 5.18).

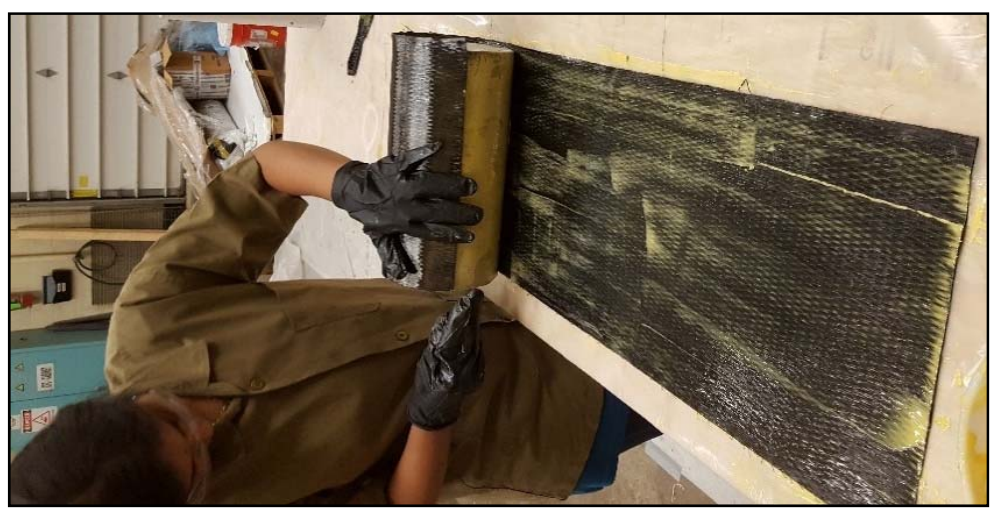

Figure 5-17 Wrapping of Concrete Cylinders with CFRP-Epoxy System

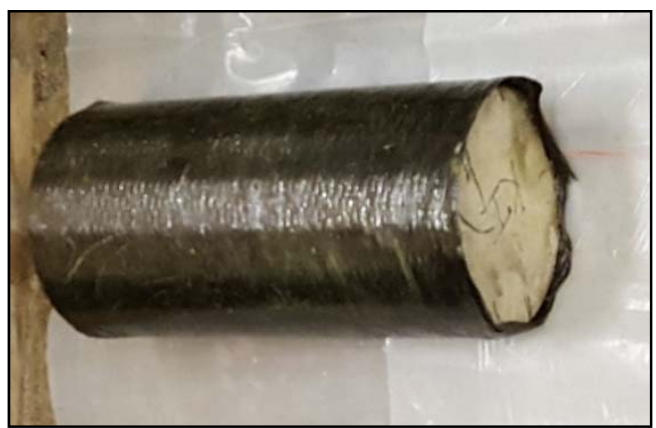

Figure 5-18 CFRP Confined Cylinder

\subsubsection{HFRP pre-preg}

i. Open the pre-impregnated hybrid fabric from the pouch and cut it into wraps as per the testing scheme (Fig 5.19).

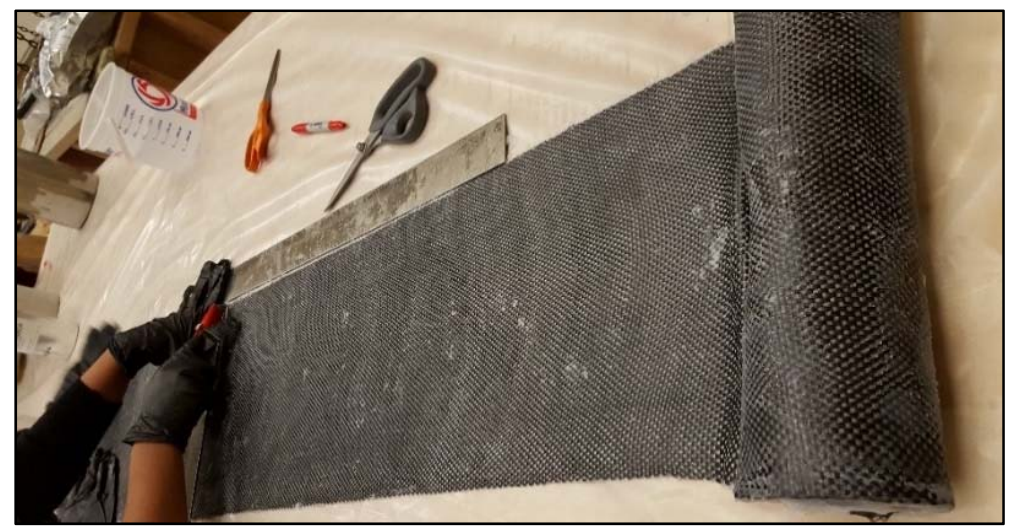

Figure 5-19 Cutting Hybrid pre-preg Fabric into Wraps 
ii. Mix the two-part primer as described in section 3.4 (Fig 5.20).
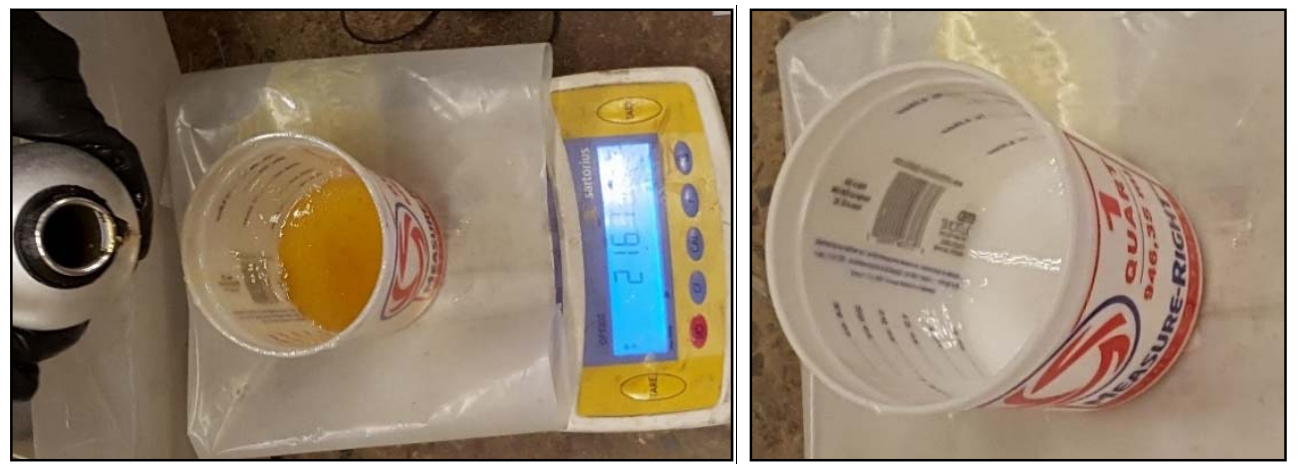

Figure 5-20 Mixing of Two-Part Primer (Left); Mixed Primer (Right)

iii. Apply primer on the surface of the concrete cylinder (Fig 5.21)

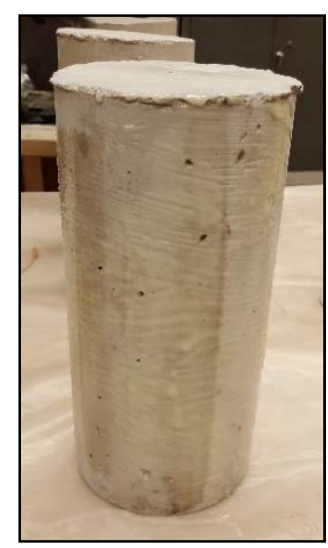

Figure 5-21 Surface of Concrete Cylinder Coated with Primer

iv. Wrap the prepared concrete cylinders with the pre-impregnated hybrid fabric (Fig 5.22).

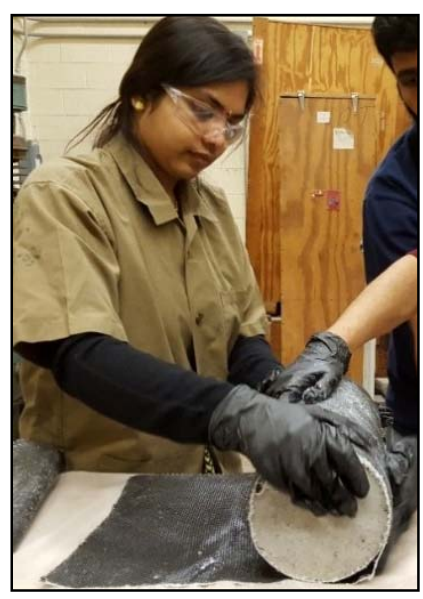

Figure 5-22 Wrapping of Concrete Cylinders with HFRP pre-preg 
v. Keep the wrapped cylinders aside with the end of the wrap lying down under the weight of the cylinder and let them cure for 5 days of curing time (Fig 5.23).

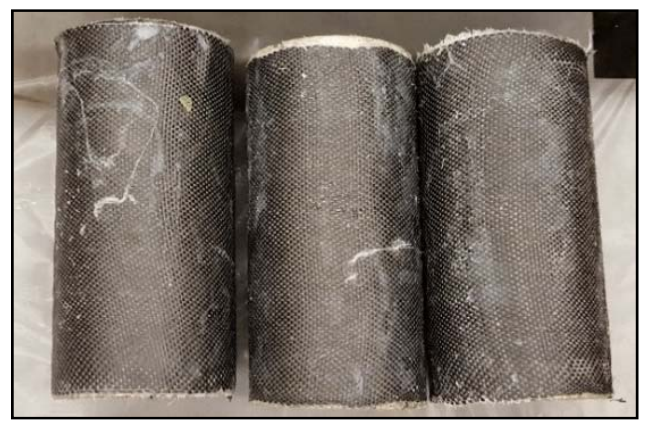

Figure 5-23 HFRP pre-preg Confined Cylinders

\subsubsection{Sulfur Capping}

Steps to prepare cylinders with sulfur capping is as given below:

i. Heat the dry sulfur flakes using melting pot to a temperature of 265 to $290 \mathrm{~F}$ (Fig 5.24).

Sulfur capping compound should be free from dampness as it may lead to foaming.

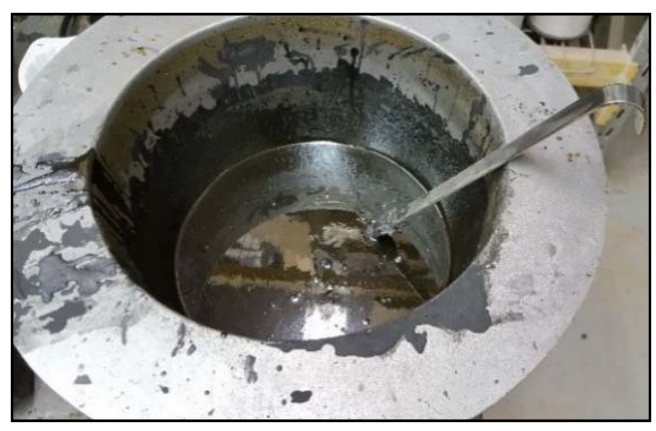

Figure 5-24 Molten Sulfur Capping Compound

ii. $\quad$ Prepare 2 in. sulfur mortar cubes prior to capping (Fig 5.25).

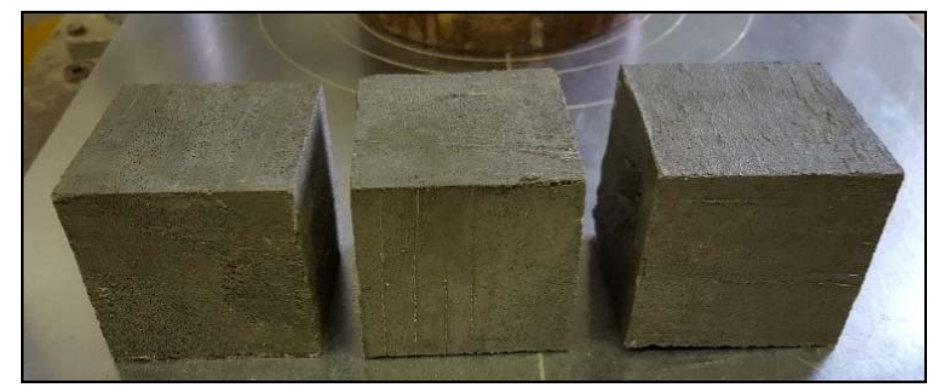

Figure 5-25 Sulfur Mortar Cubes (2 in.) 
iii. After 24 hours of curing time, determine the compressive strength of the prepared sulfur mortar cubes (Fig 5.26).

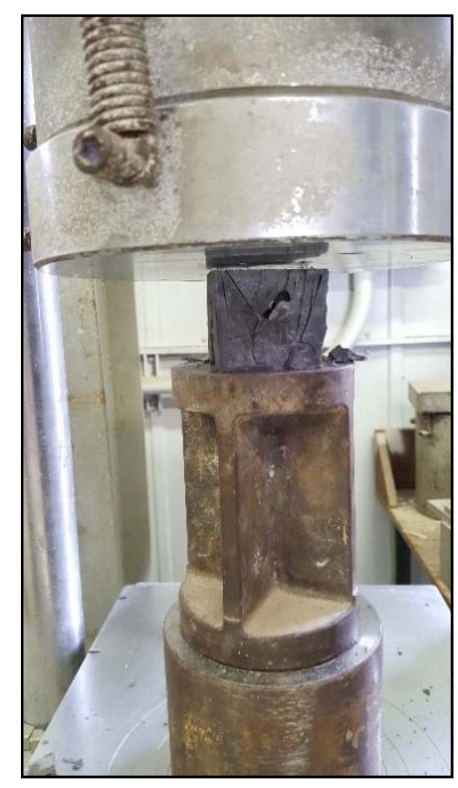

\section{Figure 5-26 Compression Test on Sulfur Mortar Cube}

iv. After obtaining the desired values of compressive strength, proceed with the capping.

v. Apply grease on the surface of the steel plate in the capping mold (Fig 5.27) to ensure easy demolding after the sulfur capping hardens.

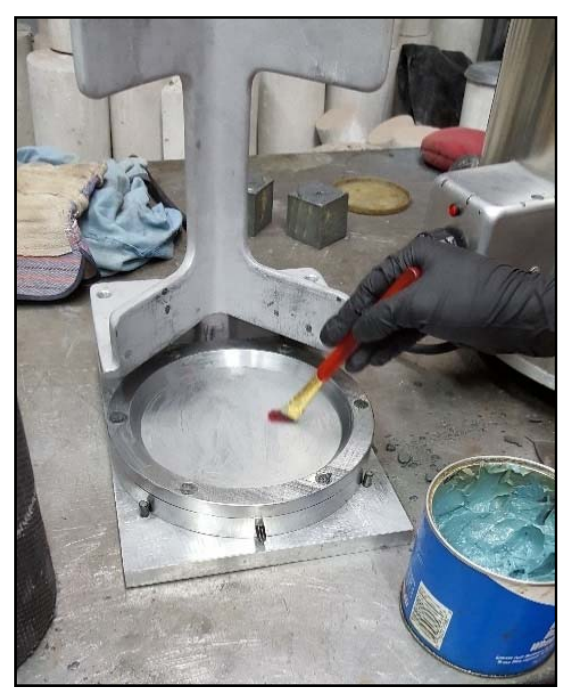

Figure 5-27 Greasing the Surface of Capping Plate

vi. Keep stirring the molten sulfur mortar prior to pouring in to the mold. 
vii. $\quad$ Pour the molten sulfur mortar in to the capping plate (Fig 5.28).

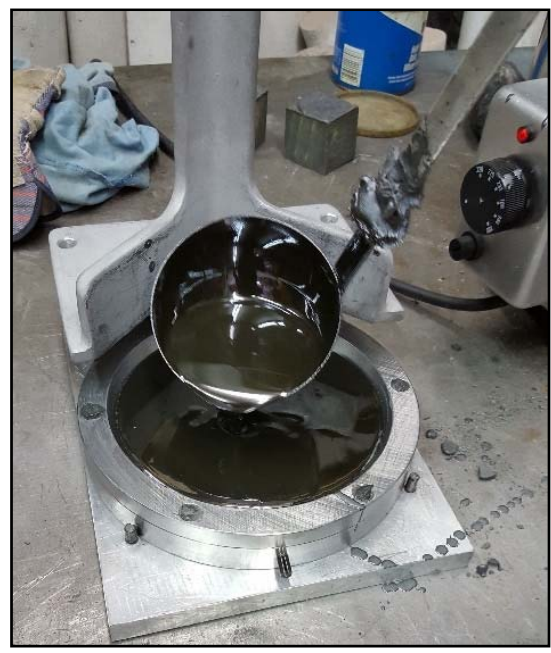

\section{Figure 5-28 Pouring Sulfur Mortar into Capping Plate}

viii. Hold the cylinder against the alignment guides of the vertical device to maintain straightness and then lower down the cylinder to rest on the mortar in the capping plate while in continuous contact with the alignment guides until the sulfur mortar gets hardened (Fig 5.29).

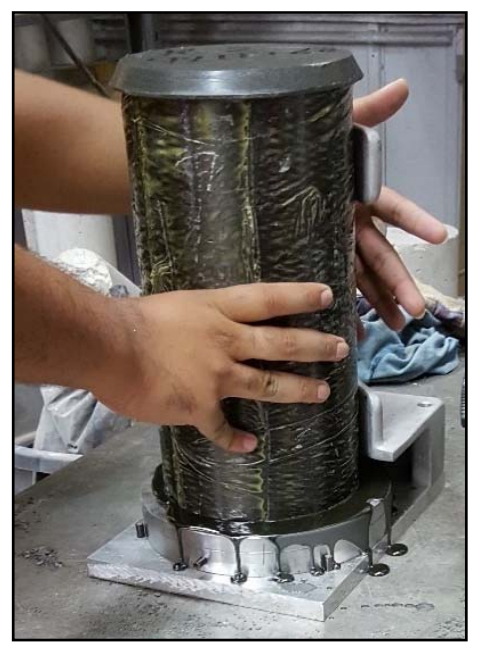

Figure 5-29 Aligning Cylinder against Vertical Device

ix. After hardening, demold the cylinder by slightly tapping the edges of the capping plate with a rubber hammer and check for straightness. 
x. Repeat the above steps for capping on the other side of the cylinder.

xi. Leave the cylinder with a wet burlap cover for 24 hours allowing the capping to gain the desired strength and then all set to test (Fig 5.30).

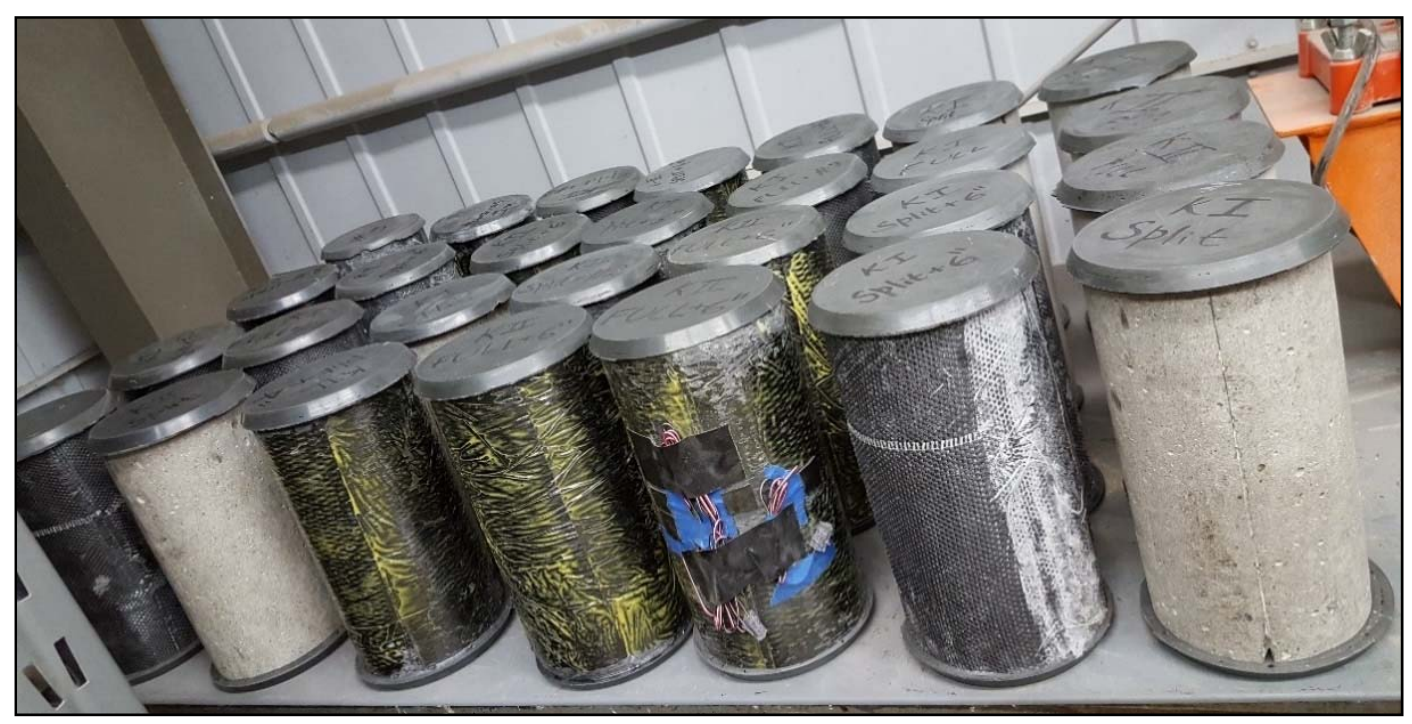

Figure 5-30 Sulfur Capped Cylinders

\subsection{Test Procedure}

Axial compression tests were performed on the concrete cylinders prepared as discussed in section 5.4 according to ASTM C39/C39M-14 using UTM as described in section 5.3. These tests were conducted to evaluate the strength, stiffness and ductility enhancements. Capped concrete cylinders were positioned at the center of the concentric circles marked on the lower mounting table of the testing frame in UTM (Fig 5.31). Capping helps in applying load uniformly throughout the specimen while maintaining concentric loading. Upper portion of the test frame is stationary whereas lower mounting table applies compression on the specimen by moving in the upward direction with the increase in application of the load. 


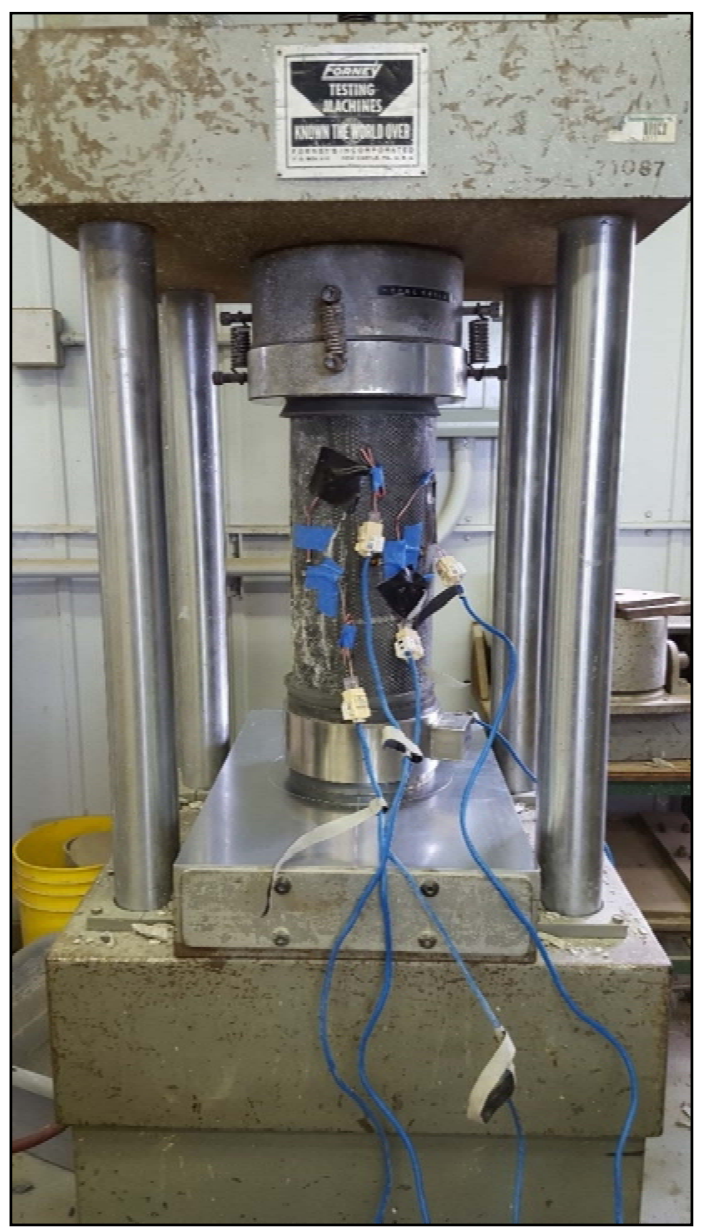

Figure 5-31 Axial Compression Testing of Concrete Cylinders

\subsection{Test Results and Analysis}

Concrete cylinders of dimension 6" x 12" were cast in two batches and were tested under axial compression in UTM as described in the above sections. Three specimens were considered in each category. Axial compression test results are tabulated in the following sections and discussions are provided by comparing load and strength of control and wrapped specimens. 


\subsubsection{Compression Tests on Full Cylinders}

\subsubsection{Compressive Strength of Non-Wrapped Full Cylinders}

Non-Wrapped Full Cylinder specimens of batch-1 and batch-2 were tested in axial compression. The failure load, average strength and average stress are tabulated in Table 5.1.

Table 5-1 Compressive Strength of Non-Wrapped Full Cylinders

\begin{tabular}{|c|c|c|c|c|}
\hline \multirow[t]{2}{*}{ Batch } & $\begin{array}{c}\text { Axial } \\
\text { Strength }\end{array}$ & $\begin{array}{c}\text { Average } \\
\text { Axial Strength }\end{array}$ & $\begin{array}{l}\text { Axial } \\
\text { Stress }\end{array}$ & $\begin{array}{c}\text { Average } \\
\text { Axial Stress } \\
\left(\mathbf{f}_{\mathbf{c}}{ }^{\prime}\right)\end{array}$ \\
\hline & (kip) & (kip) & (ksi) & (ksi) \\
\hline \multirow{3}{*}{ Batch 1} & 152 & \multirow{3}{*}{154} & 5.388 & \multirow{3}{*}{5.46} \\
\hline & 158 & & 5.588 & \\
\hline & 153 & & 5.394 & \\
\hline \multirow{3}{*}{ Batch 2} & 180 & \multirow{3}{*}{181} & 6.37 & \multirow{3}{*}{6.40} \\
\hline & 178 & & 6.3 & \\
\hline & 185 & & 6.54 & \\
\hline
\end{tabular}

Batch-1 and batch-2 non-wrapped full cylinder specimens failed at average axial compressive stress values of $5.46 \mathrm{ksi}$ and $6.40 \mathrm{ksi}$ respectively.

\subsubsection{Compressive Strength of Wrapped Full Cylinders}

The axial compressive strength and stress values of confined cylinders with CFRP system in one, two and three layers wrapping scheme are presented in Table 5.2 for batch-1 and Table 5.3 for batch-2. The results with HFRP pre-preg confinement are presented in Table 5.4 for batch-1.

The tabulated values are compared to the values of non-wrapped full cylinder specimens of the respective batches to determine the strength increase due to confinement effect. 
Table 5-2 Compressive Strength of CFRP Wrapped Full Cylinders from Batch-1

\begin{tabular}{|c|c|c|c|c|c|c|c|c|}
\hline \multirow{2}{*}{ FRP } & \multirow{2}{*}{$\begin{array}{l}\text { No. of } \\
\text { Layers }\end{array}$} & $\begin{array}{c}\text { Axial } \\
\text { Strength }\end{array}$ & $\begin{array}{c}\text { Avg. } \\
\text { Axial } \\
\text { Strength } \\
\end{array}$ & Stress & $\begin{array}{l}\text { Avg. } \\
\text { Stress } \\
\left(\mathrm{f}_{\mathrm{c}}\right) \\
\end{array}$ & \multirow{2}{*}{$\begin{array}{c}\text { Avg. } \\
\text { Strength } \\
\text { Increase } \\
(\%)\end{array}$} & \multirow{2}{*}{$\begin{array}{c}\text { Avg } \\
\text { Strength } \\
\text { Increase } \\
\text { per } \\
\text { layer } \\
(\%)\end{array}$} & \multirow{2}{*}{$\begin{array}{c}\text { Strength } \\
\text { Ratio }\end{array}$} \\
\hline & & (kip) & (kip) & (ksi) & (ksi) & & & \\
\hline \multirow{12}{*}{ CFRP } & \multirow{3}{*}{0} & 152 & \multirow{3}{*}{154} & 5.39 & \multirow{3}{*}{5.46} & \multirow{3}{*}{ - } & \multirow{3}{*}{ - } & \multirow{3}{*}{ - } \\
\hline & & 158 & & 5.59 & & & & \\
\hline & & 153 & & $\begin{array}{l}5.39 \\
\end{array}$ & & & & \\
\hline & \multirow{3}{*}{1} & 239 & \multirow{3}{*}{258} & 8.45 & \multirow{3}{*}{9.11} & \multirow{3}{*}{67} & \multirow{3}{*}{67} & \multirow{3}{*}{1.67} \\
\hline & & 252 & & 8.91 & & & & \\
\hline & & 282 & & 9.97 & & & & \\
\hline & \multirow{3}{*}{2} & $286^{*}$ & \multirow{3}{*}{428} & 10.12 & \multirow{3}{*}{15.14} & \multirow{3}{*}{177} & \multirow{3}{*}{89} & \multirow{3}{*}{2.77} \\
\hline & & 426 & & 15.07 & & & & \\
\hline & & 430 & & $\begin{array}{l}15.21 \\
\end{array}$ & & & & \\
\hline & \multirow{3}{*}{3} & 485 & \multirow{3}{*}{492} & 17.15 & \multirow{3}{*}{17.40} & \multirow{3}{*}{219} & \multirow{3}{*}{73} & \multirow{3}{*}{3.19} \\
\hline & & $495^{+}$ & & 17.51 & & & & \\
\hline & & $496^{+}$ & & 17.54 & & & & \\
\hline
\end{tabular}

*Outlier not considered in calculating average values

${ }^{+}$No failure observed and could not load beyond as the machine reached its loading limit

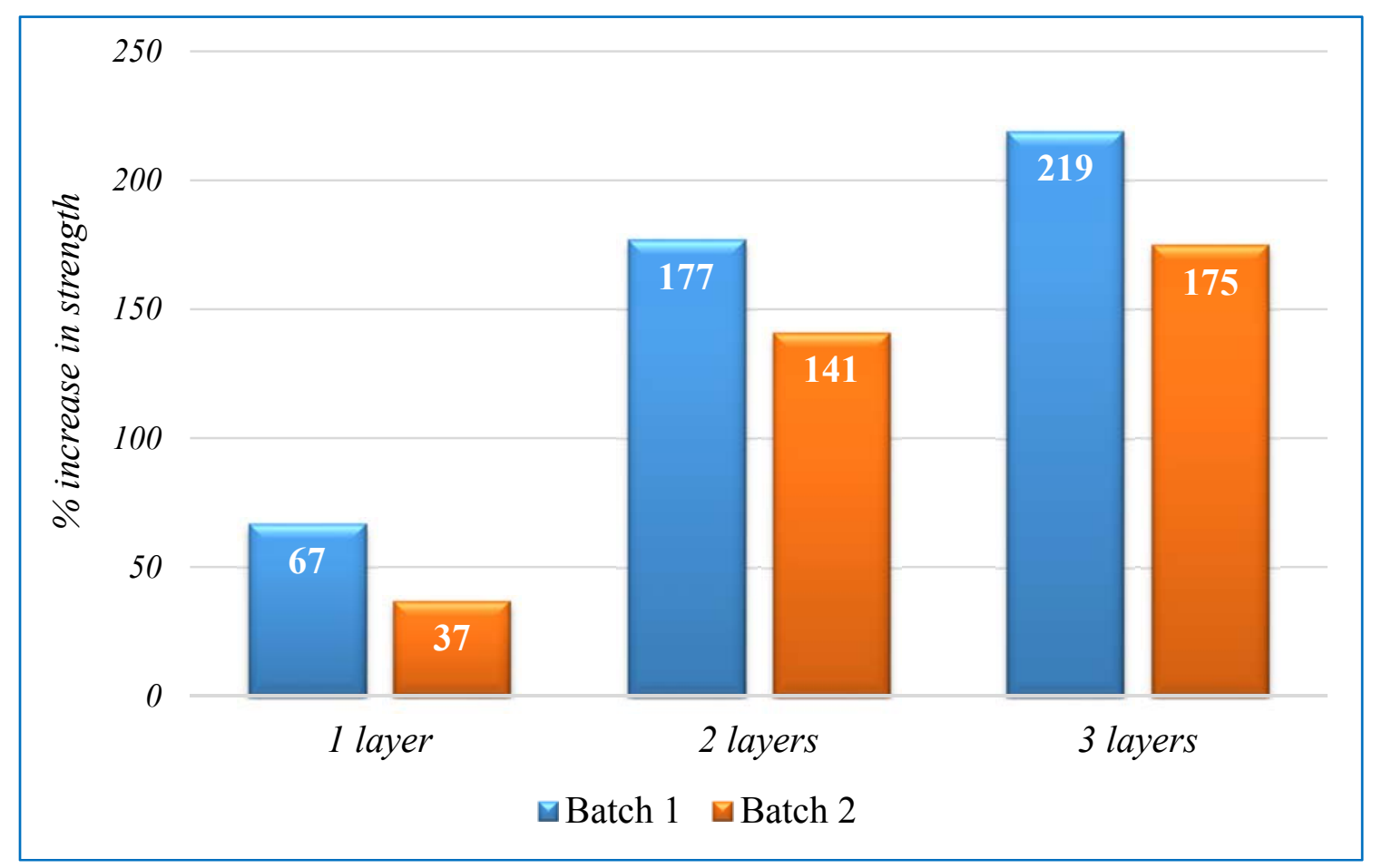

Figure 5-32 Average Axial Strength Increase for 1, 2, \& 3 Layers of Wrapping with CFRP 
Table 5-3 Compressive Strength of CFRP Wrapped Full Cylinders from Batch-2

\begin{tabular}{|c|c|c|c|c|c|c|c|c|}
\hline \multirow[t]{2}{*}{ FRP } & \multirow[t]{2}{*}{$\begin{array}{l}\text { No. of } \\
\text { Layers }\end{array}$} & $\begin{array}{c}\text { Axial } \\
\text { Strength }\end{array}$ & $\begin{array}{c}\text { Avg. } \\
\text { Axial } \\
\text { Strength }\end{array}$ & Stress & $\begin{array}{c}\text { Avg. } \\
\text { Stress } \\
\left(f_{\text {cc' }}\right)\end{array}$ & \multirow{2}{*}{$\begin{array}{c}\text { Avg. } \\
\text { Strength } \\
\text { Increase } \\
(\%)\end{array}$} & \multirow{2}{*}{$\begin{array}{l}\text { Avg. } \\
\text { Strength } \\
\text { Increase } \\
\text { per } \\
\text { layer } \\
(\%)\end{array}$} & \multirow[t]{2}{*}{$\begin{array}{c}\text { Strength } \\
\text { Ratio }\end{array}$} \\
\hline & & (kip) & (kip) & (ksi) & (ksi) & & & \\
\hline \multirow{12}{*}{ CFRP } & \multirow{3}{*}{0} & 180 & \multirow{3}{*}{181} & 6.37 & \multirow{3}{*}{6.40} & \multirow{3}{*}{-} & \multirow{3}{*}{ - } & \multirow{3}{*}{ - } \\
\hline & & 178 & & 6.30 & & & & \\
\hline & & 185 & & 6.54 & & & & \\
\hline & \multirow{3}{*}{1} & 244 & \multirow{3}{*}{248} & 8.63 & \multirow{3}{*}{8.77} & \multirow{3}{*}{37} & \multirow{3}{*}{37} & \multirow{3}{*}{1.37} \\
\hline & & 250 & & 8.86 & & & & \\
\hline & & 249 & & 8.82 & & & & \\
\hline & \multirow{3}{*}{2} & $230 *$ & \multirow{3}{*}{437} & 8.13 & \multirow{3}{*}{15.46} & \multirow{3}{*}{141} & \multirow{3}{*}{71} & \multirow{3}{*}{2.41} \\
\hline & & 424 & & 15.00 & & & & \\
\hline & & 450 & & 15.92 & & & & \\
\hline & \multirow{3}{*}{3} & $450 *$ & \multirow{3}{*}{497} & 15.92 & \multirow{3}{*}{17.58} & \multirow{3}{*}{175} & \multirow{3}{*}{58} & \multirow{3}{*}{2.75} \\
\hline & & $498^{+}$ & & 17.61 & & & & \\
\hline & & $496^{+}$ & & 17.54 & & & & \\
\hline
\end{tabular}

*Outlier not considered in calculating average values

${ }^{+}$No failure observed and could not load beyond as the machine reached its loading limit

* One layer and two layer CFRP wrapped cylinders from batch-1 observed an average strength increase of $67 \%$ and $177 \%$ respectively as compared to the control specimens of batch-1.

- One layer and two layer CFRP wrapped cylinders from batch-2 observed an average strength increase of $37 \%$ and $141 \%$ respectively as compared to the control specimens of batch-2.

* Three layer wrapped cylinders from both the batches could not be tested until failure due to their strength being exceeded the 500 kip capacity of the UTM. 
Table 5-4 Compressive Strength of HFRP pre-preg Wrapped Full Cylinders from Batch-1

\begin{tabular}{|c|c|c|c|c|c|c|c|c|}
\hline \multirow[t]{2}{*}{ FRP } & \multirow[t]{2}{*}{$\begin{array}{l}\text { No. of } \\
\text { Layers }\end{array}$} & $\begin{array}{c}\text { Axial } \\
\text { Strength }\end{array}$ & $\begin{array}{c}\text { Avg. } \\
\text { Axial } \\
\text { Strength } \\
\end{array}$ & Stress & $\begin{array}{l}\text { Avg. } \\
\text { Stress } \\
\left(\mathrm{f}_{\mathrm{cc}}\right) \\
\end{array}$ & \multirow{2}{*}{$\begin{array}{l}\text { Avg. } \\
\text { Strength } \\
\text { Inc. (\%) }\end{array}$} & \multirow{2}{*}{$\begin{array}{l}\text { Avg. } \\
\text { Strength } \\
\text { inc. per } \\
\text { layer } \\
(\%)\end{array}$} & \multirow[t]{2}{*}{$\begin{array}{c}\text { Strength } \\
\text { Ratio }\end{array}$} \\
\hline & & (kip) & (kip) & (ksi) & (ksi) & & & \\
\hline \multirow{10}{*}{ HFRP } & 0 & - & 154 & - & 5.46 & - & - & - \\
\hline & \multirow{3}{*}{1} & 206 & \multirow[t]{3}{*}{206} & 7.27 & \multirow{3}{*}{7.29} & \multirow{3}{*}{34} & \multirow{3}{*}{34} & \multirow{3}{*}{1.34} \\
\hline & & 211 & & 7.45 & & & & \\
\hline & & 202 & & 7.16 & & & & \\
\hline & \multirow{3}{*}{2} & 276 & \multirow{3}{*}{268} & 9.76 & \multirow{3}{*}{9.47} & \multirow{3}{*}{74} & \multirow{3}{*}{37} & \multirow{3}{*}{1.74} \\
\hline & & 258 & & 9.11 & & & & \\
\hline & & 270 & & 9.55 & & & & \\
\hline & \multirow{3}{*}{3} & 382 & \multirow{3}{*}{367} & 13.51 & \multirow{3}{*}{12.97} & \multirow{3}{*}{138} & \multirow{3}{*}{46} & \multirow{3}{*}{2.03} \\
\hline & & 360 & & 12.73 & & & & \\
\hline & & 358 & & 12.66 & & & & \\
\hline
\end{tabular}

One, two and three layer HFRP pre-preg wrapped cylinders from batch-1 observed an average strength increase of $34 \%, 74 \%$ and $138 \%$ respectively comparing to the control specimens of batch-1.

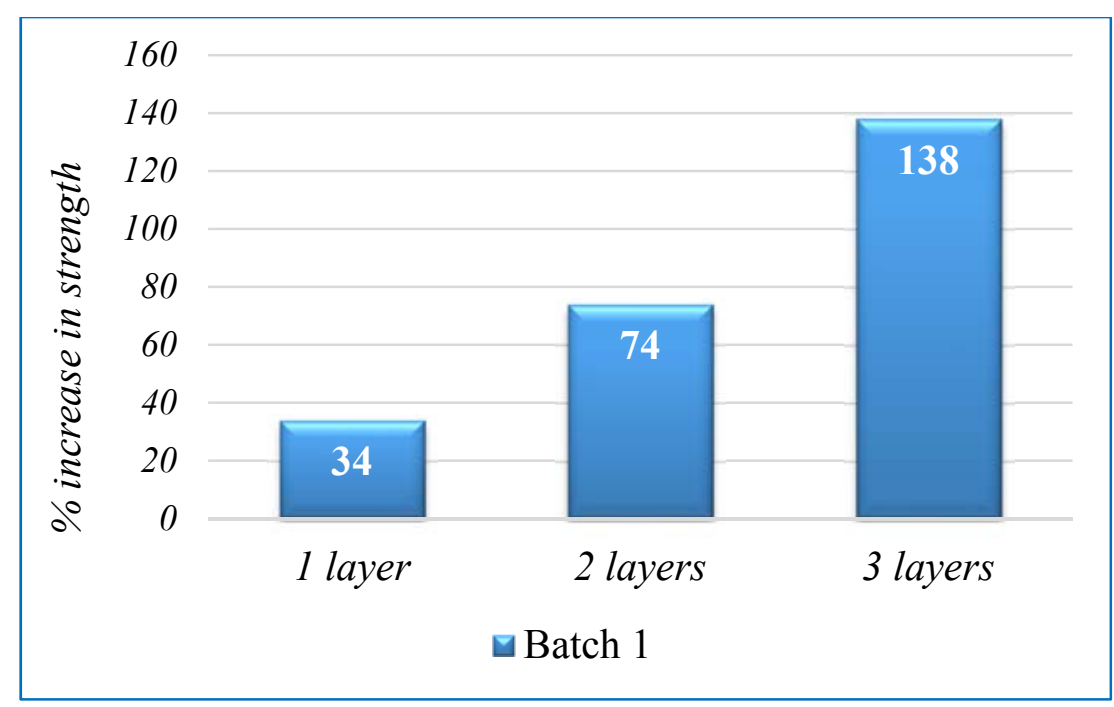

Figure 5-33 Average Axial Strength Increase for 1, 2, \& 3 Layers of Wrapping with HFRP pre-preg 


\subsubsection{Stress-Strain Curves of Full Cylinders}

Cylinder specimens were tested under axial compression, load and strain data were collected through data acquisition system. Strain gage was mounted on the fabric for the wrapped cylinders and on concrete for the non-wrapped cylinders. Stress calculated from the obtained load data and plotted against strain data. The typical stress-strain curve plotted for the full cylinders is shown in Fig. 5.34.

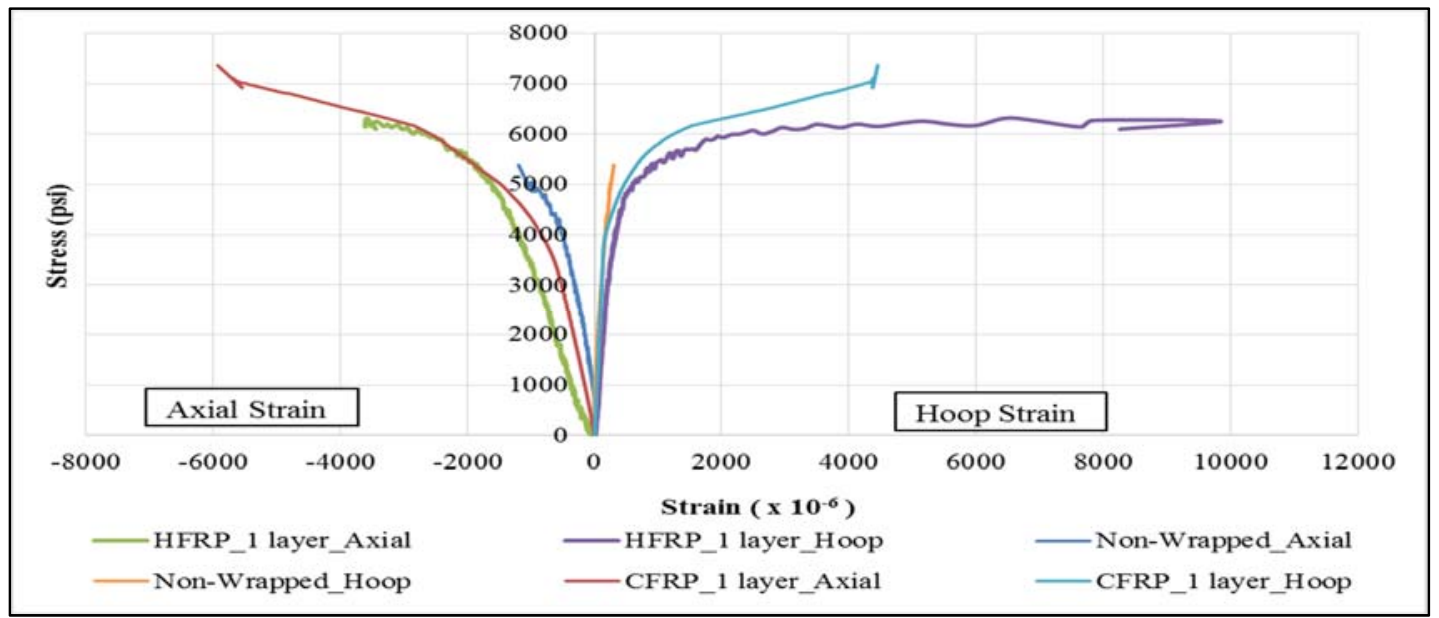

Note: Biaxial fabrics (Hybrid with carbon in hoop direction and glass in axial direction) could exhibit higher axial strains based on resin dominant axial behavior and bond characteristics between concrete and wrap.

Figure 5-34 Stress-Strain Curves for Non-Wrapped Full Cylinders, 1Layer CFRP and HFRP Wrapped Full Cylinders of Batch-1

\subsubsection{Theoretical Analysis of Full Cylinders}

The experimental results obtained from testing 1,2 and 3 layer FRP confined concrete cylinders are correlated with analytical model given in ACI 440.2R-08.

According to ACI 440.2R-08,

Where,

$$
\frac{f_{c c}^{\prime}}{f_{c o}^{\prime}}=1+3.3 \frac{f_{l}}{f_{c o}^{\prime}}
$$

$\boldsymbol{f}_{c c}^{\prime}=$ compressive strength of confined concrete

$\boldsymbol{f}_{\boldsymbol{c o}}^{\prime}=$ compressive strength of unconfined concrete 


$$
\begin{aligned}
& \boldsymbol{f}_{\boldsymbol{l}}=\text { lateral confined pressure by FRP wraps }=\frac{\boldsymbol{2}_{\boldsymbol{f}} \boldsymbol{n} \boldsymbol{t}_{\boldsymbol{f}} \boldsymbol{\varepsilon}_{\boldsymbol{f}}}{\boldsymbol{D}} \\
& \boldsymbol{E}_{\boldsymbol{f}}=\text { modulus of elasticity of FRP } \\
& \mathrm{n}=\text { number of layers }=1,2 \text {, and } 3 \\
& \boldsymbol{t}_{\boldsymbol{f}}=\text { thickness of fabric } \\
& \text { D }=\text { diameter of cylinder } \\
& \boldsymbol{\varepsilon}_{\boldsymbol{f} \boldsymbol{e}}=\text { effective failure strain of fabric }=\boldsymbol{k}_{\boldsymbol{\varepsilon}} \boldsymbol{\varepsilon}_{\boldsymbol{f} \boldsymbol{u}} \\
& \boldsymbol{k}_{\boldsymbol{\varepsilon}}=\text { strain efficiency factor }=0.55 \\
& \boldsymbol{\varepsilon}_{\boldsymbol{f} \boldsymbol{u}}=\text { ultimate failure strain of fabric }
\end{aligned}
$$

\begin{tabular}{|c|c|c|c|c|c|c|}
\hline FRP & $\begin{array}{c}\text { No. of } \\
\text { Layers }\end{array}$ & $\begin{array}{c}f_{c o}^{\prime} \\
\text { (psi) }\end{array}$ & $\begin{array}{c}\boldsymbol{f}_{l}^{\prime} \\
(\mathrm{psi})\end{array}$ & $\begin{array}{c}\left(f_{c c}^{\prime}\right)_{\exp } \\
(\mathrm{psi})\end{array}$ & $\begin{array}{c}\left(\boldsymbol{f}_{c c}^{\prime}\right)_{\text {theory }} \\
(\mathrm{psi})\end{array}$ & $\frac{\left(f_{c c}^{\prime}\right)_{\text {exp }}}{\left(f_{c c}^{\prime}\right)_{\text {theory }}}$ \\
\hline \multirow{3}{*}{ CFRP } & 1 & \multirow{6}{*}{5460} & 986 & 258 & 246 & 1.05 \\
\hline & 2 & & 1971 & 428 & 338 & 1.27 \\
\hline & 3 & & 2957 & 492 & 430 & 1.14 \\
\hline \multirow{3}{*}{ HFRP } & 1 & & 460 & 206 & 197 & 1.04 \\
\hline & 2 & & 919 & 268 & 240 & 1.12 \\
\hline & 3 & & 1379 & 367 & 283 & 1.30 \\
\hline \multirow{3}{*}{ CFRP } & 1 & \multirow{3}{*}{6400} & 986 & 248 & 273 & 0.91 \\
\hline & 2 & & 1971 & 437 & 365 & 1.20 \\
\hline & 3 & & 2957 & 497 & 457 & 1.09 \\
\hline
\end{tabular}

According to calculations, the test results were within 10 to $30 \%$ of the theoretical values. The theoretical values and comparison with experimental results are tabulated in Table 5.5. The experimental to theoretical ratios for one layered cylinders ranged from 0.91 to 1.05 , for two and three layered cylinders 1.09 to 1.30 . This shows that one layer is not very effective because of lesser confinement than multi layers and hence are very effective showing higher values than theoretical calculations.

Table 5-5 Theoretical Analysis of FRP Confined Concrete Cylinders 


\subsubsection{Failure Modes of Full Cylinders}

The failure of non-wrapped full cylinders from batch-1 and batch- 2 was brittle. The failure modes varied from well-formed cone on one end to columnar vertical cracking through both ends with no well-formed cones which are Type 2 and Type 3 failure modes as per ASTM C39.

Full cylinders of batch-1 and batch-2 wrapped with 1, 2, and 3 layers of CFRP-epoxy system failed with failure modes varied among well-formed cones on both sides or one side and columnar vertical cracking through both ends with no well-formed cones which are Type 1, Type 2 and Type 3 failure modes as per ASTM C39. Cylinders with 1 layer exhibited failures similar to that of nonwrapped cylinders but with better ductility, whereas two layer CFRP wrapped full cylinders resulted in improved ductility and deformability. Wrap was observed to rupture after the concrete had been fragmented and the failure occurred near the top and bottom ends of the cylinder. Three layer CFRP wrapped full cylinders showed the highest ductility and two of the three specimens from each batch for 3 layers did not fail as the load reached the maximum capacity of the UTM.

Full cylinders of batch-1 wrapped with 1, 2 and 3 layers of HFRP Pre-preg system failed in a brittle manner with buckling being observed mostly near the center of the specimens and with rupture of the fabric at the location of bulging of concrete. On the other hand, in some of the specimens, eventual failure was observed with the de-bonding of overlap in the FRP wrap. Fibers with required thickness and stiffness in axial direction helps in preventing buckling and allow fibers in hoop direction to provide necessary confinement. Use of hybrid fabrics can help achieve the strength enhancement in an economical manner.

The failure modes of all the full cylinders are shown below in Fig 5.35 to 5.45. 

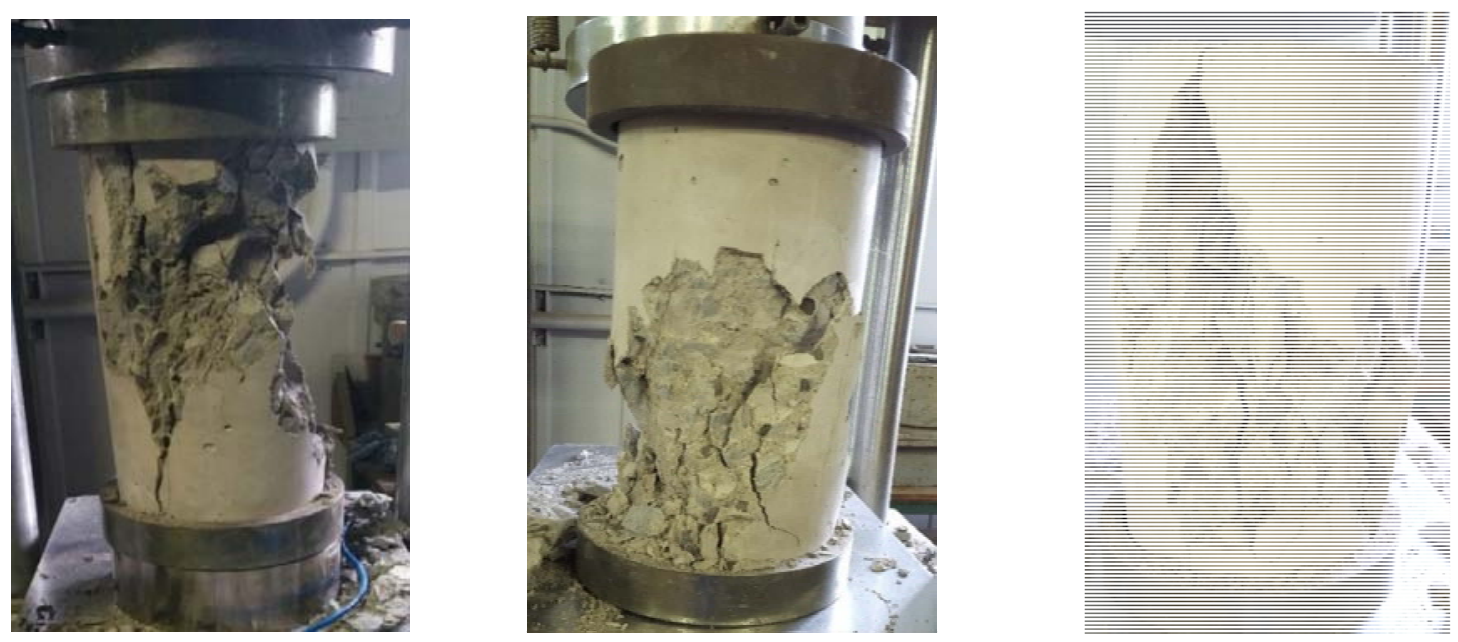

Figure 5-35 Failure Modes of Non-Wrapped Full Cylinders from Batch-1
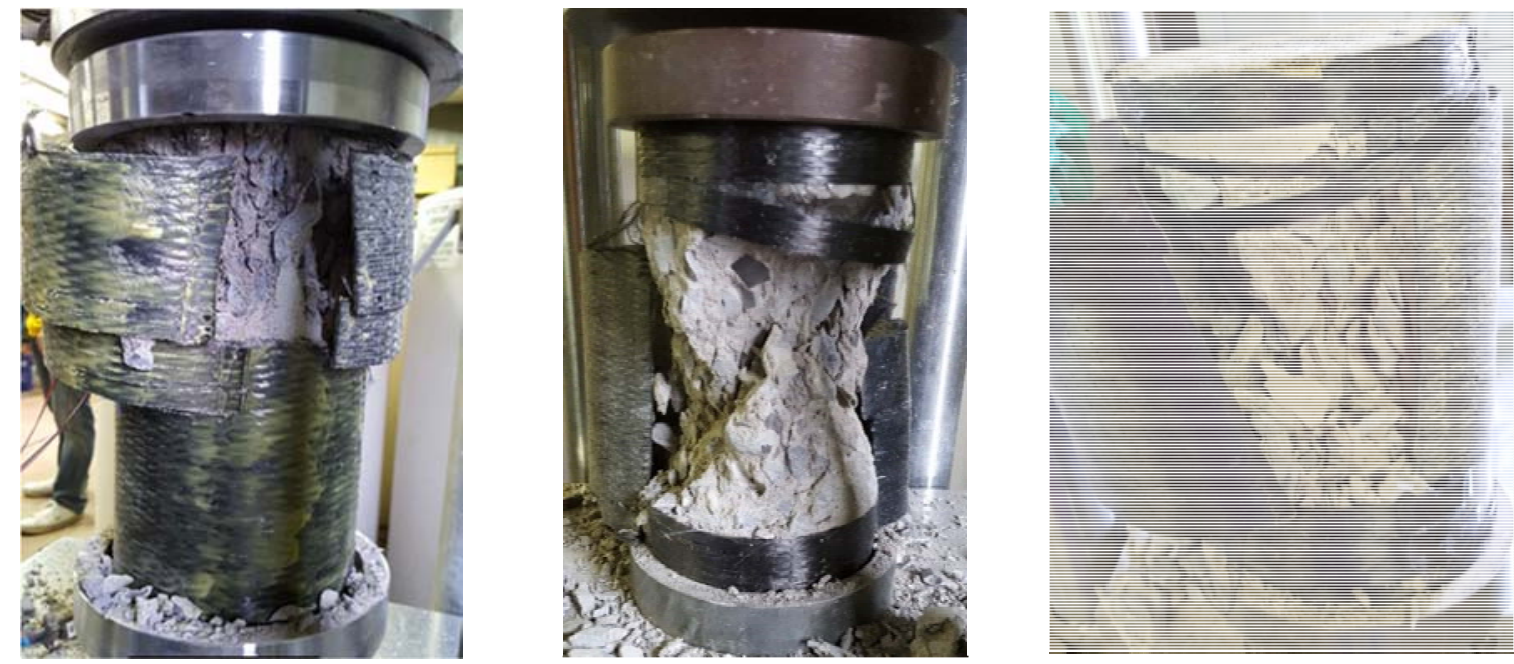

Figure 5-36 Failure Modes of 1 Layer CFRP Wrapped Full Cylinders from Batch-1

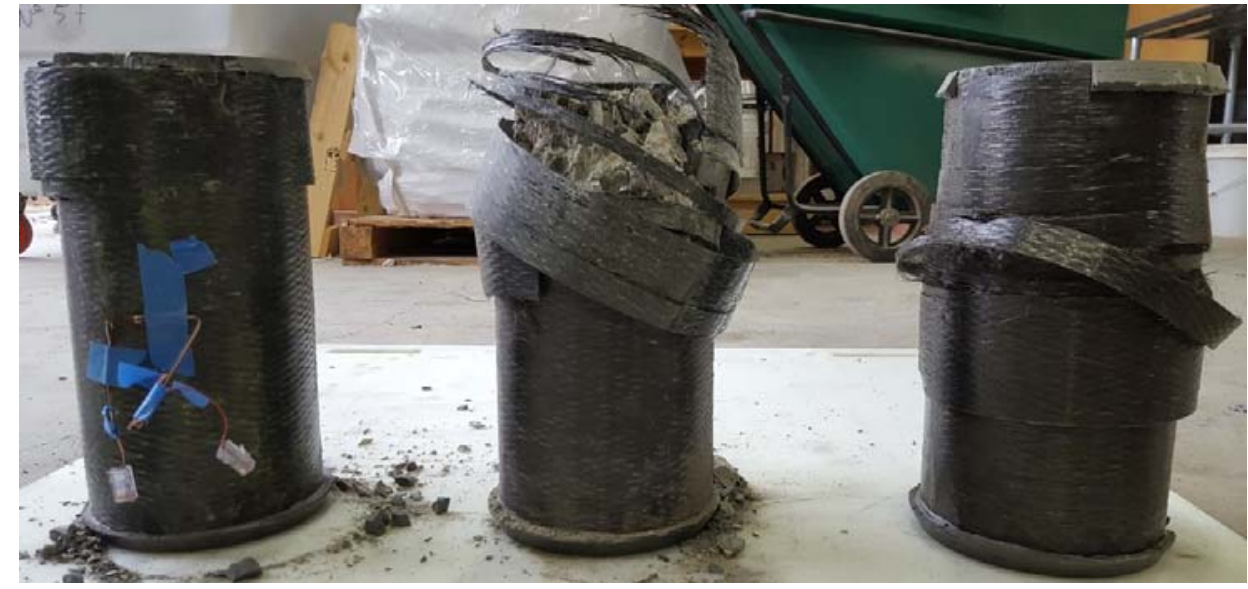

Figure 5-37 Failure Modes of 2 Layer CFRP Wrapped Full Cylinders from Batch-1 


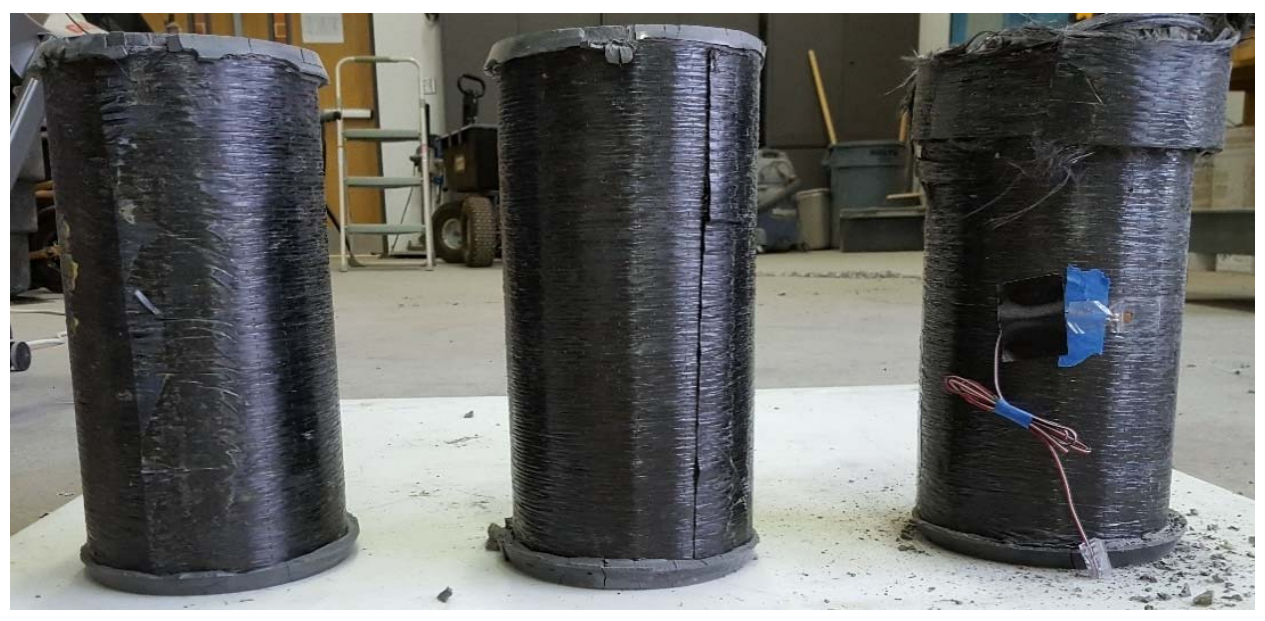

Figure 5-38 Failure Modes of 3 Layer CFRP Wrapped Full Cylinders from Batch-1
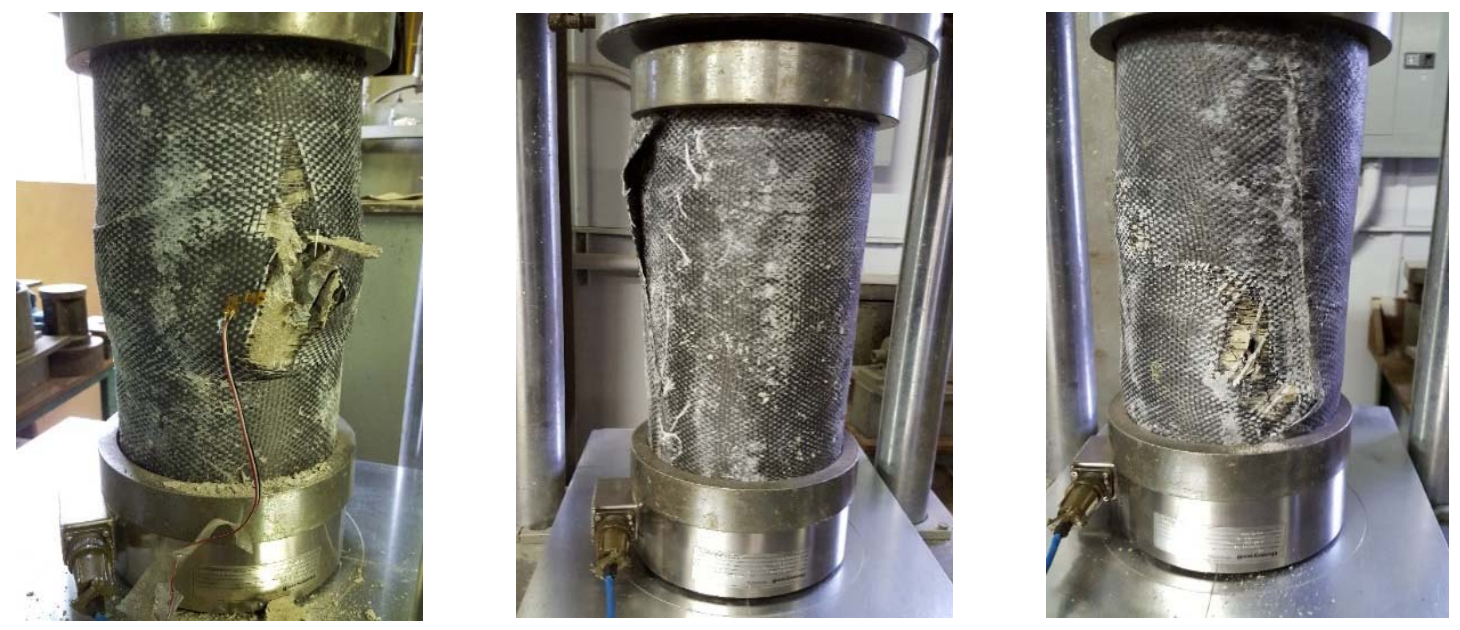

Figure 5-39 Failure Modes of 1 Layer HFRP Pre-preg Wrapped Full Cylinders from Batch-1
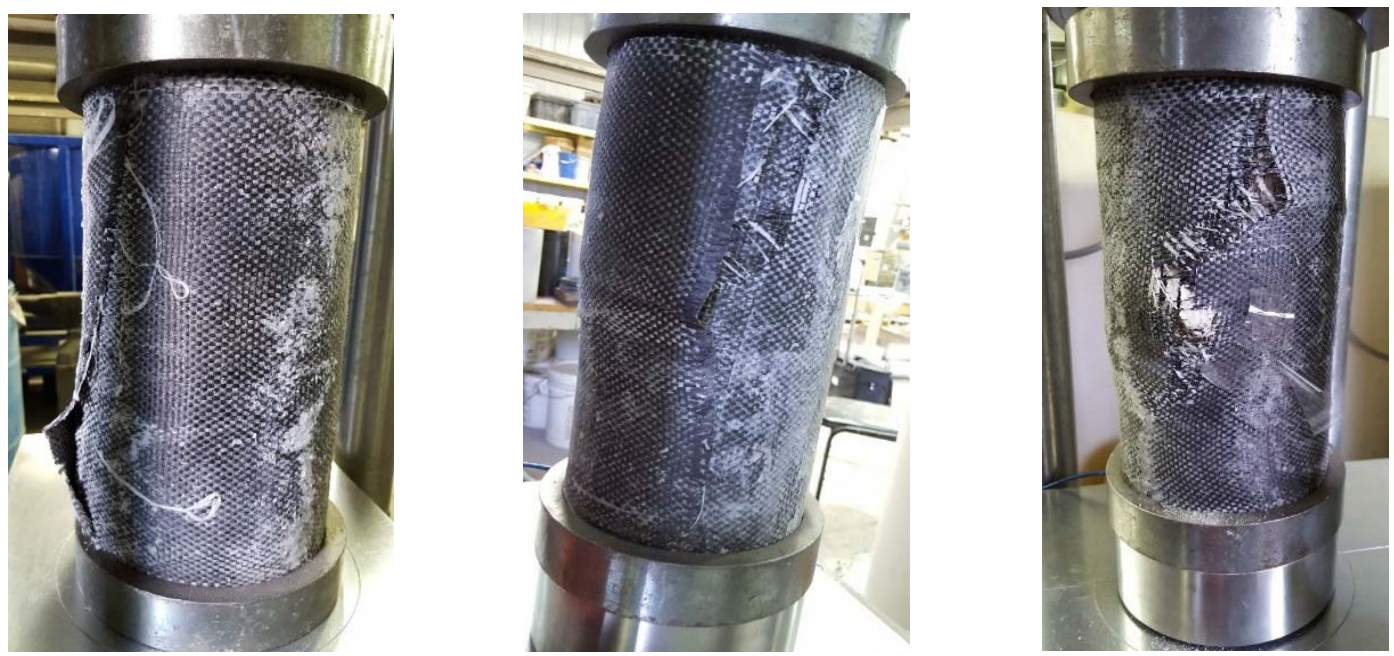

Figure 5-40 Failure Modes of 2 Layer HFRP Pre-preg Wrapped Full Cylinders from Batch-1 


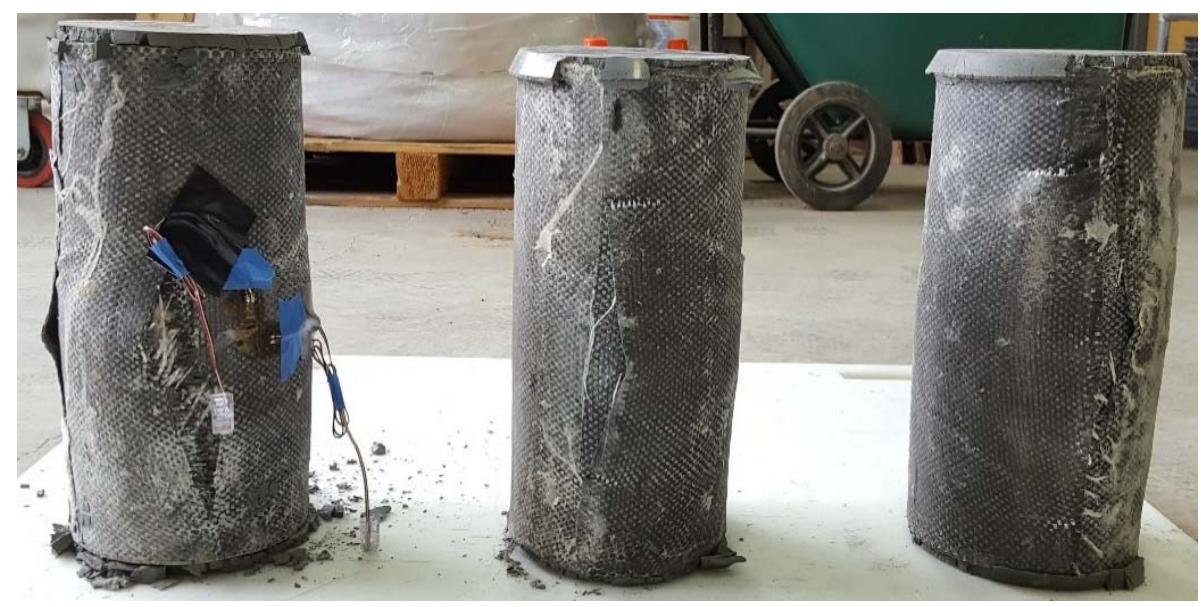

Figure 5-41 Failure Modes of 3 Layer HFRP Pre-preg Wrapped Full Cylinders from Batch-1
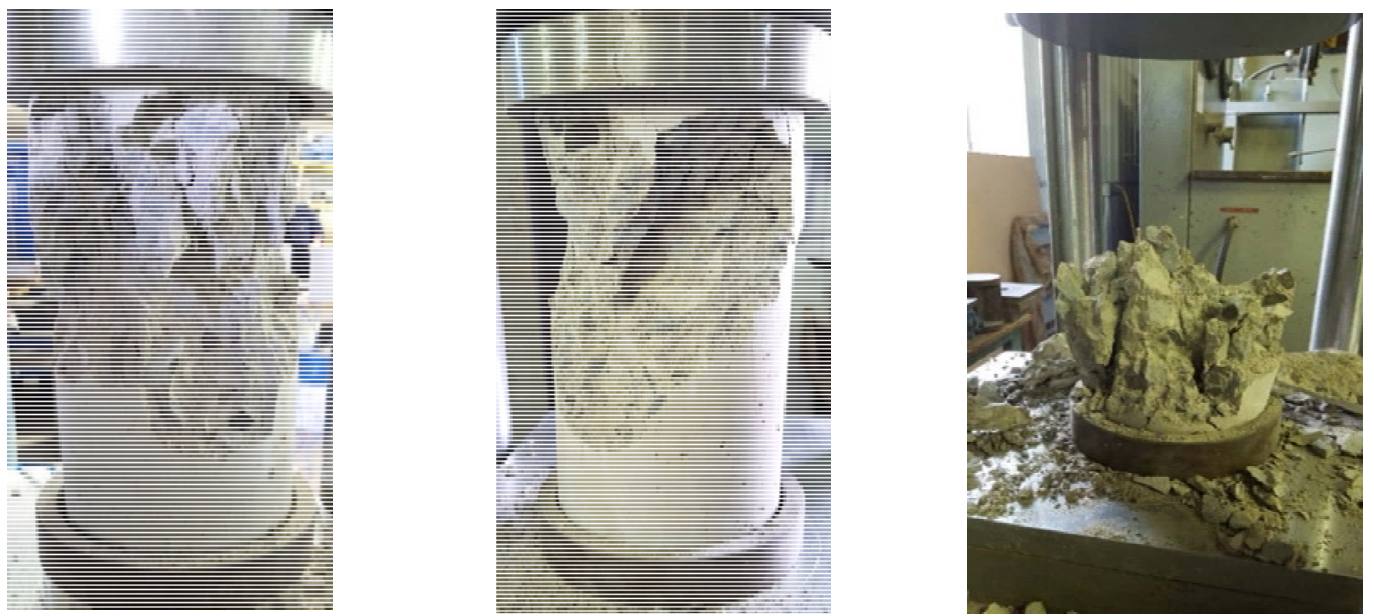

Figure 5-42 Failure Modes of Non-Wrapped Full Cylinders from Batch-2
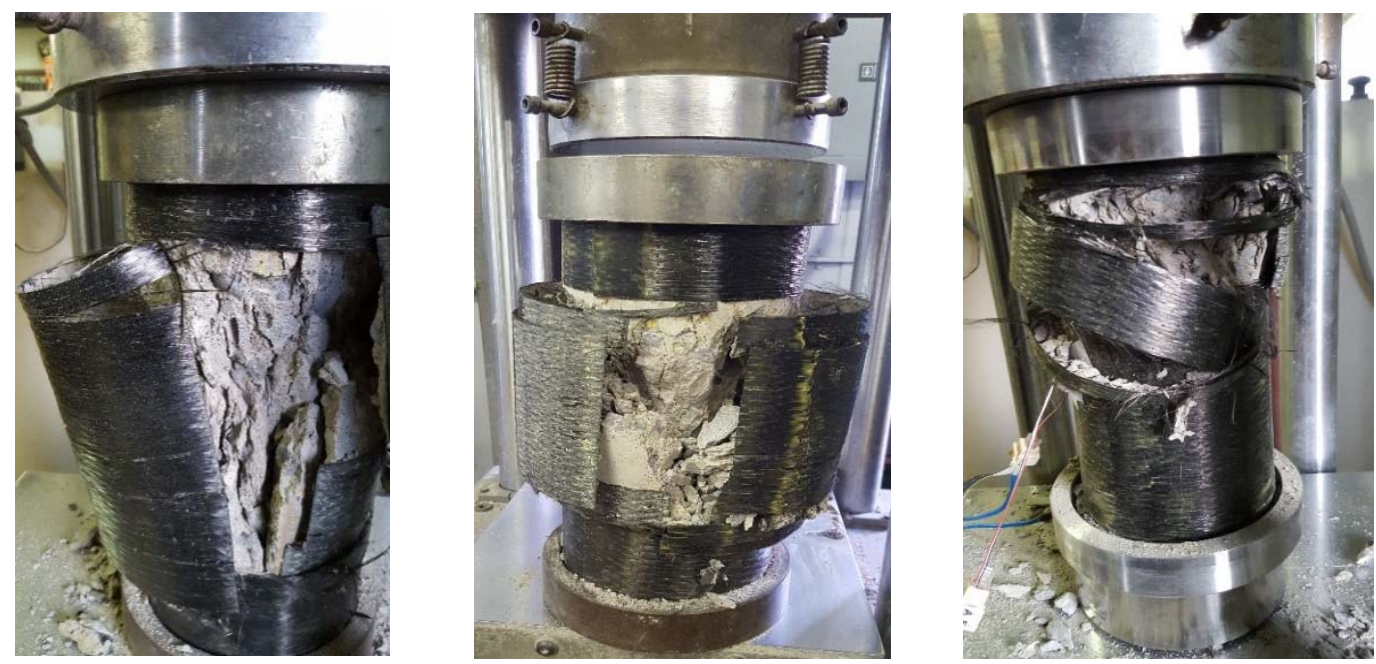

Figure 5-43 Failure Modes of 1 Layer CFRP Wrapped Full Cylinders from Batch-2 


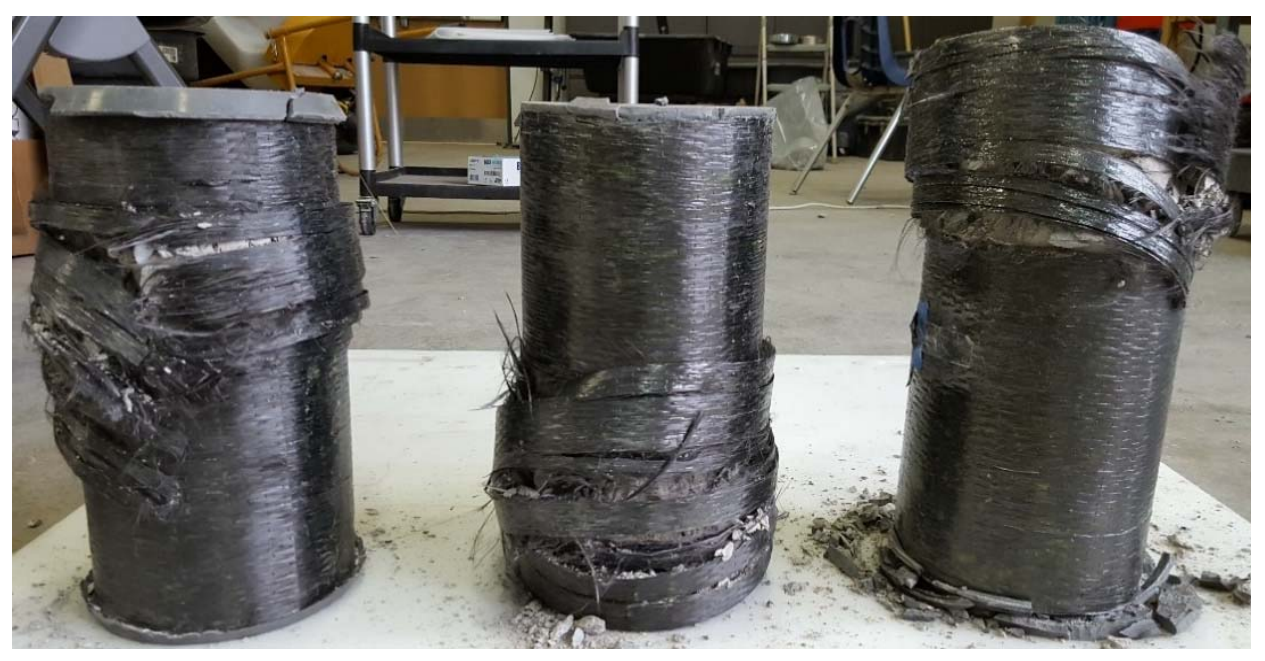

Figure 5-44 Failure Modes of 2 Layer CFRP Wrapped Full Cylinders from Batch-2

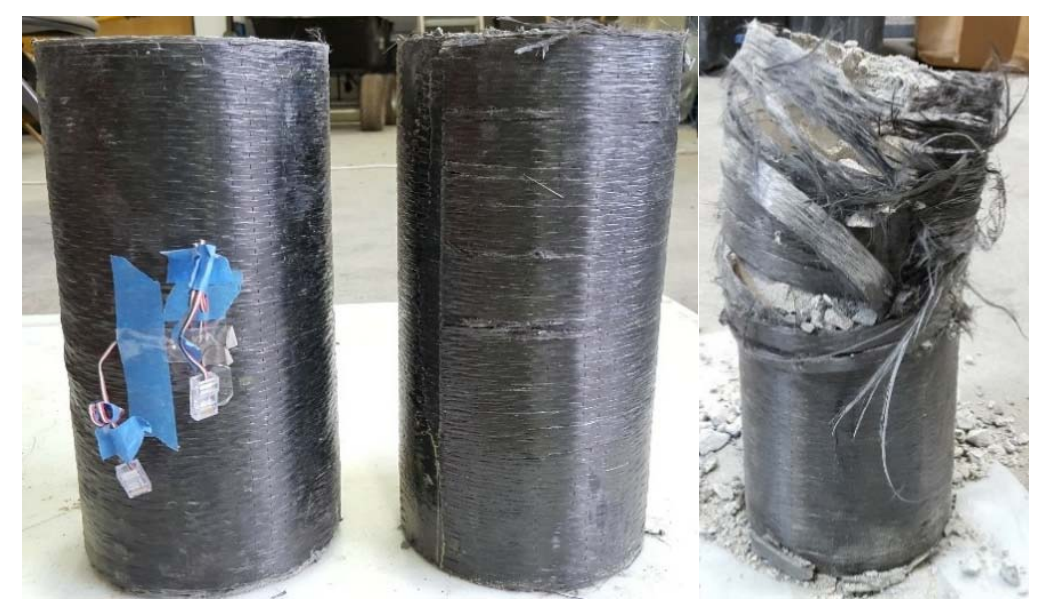

Figure 5-45 Failure Modes of 3 Layer CFRP Wrapped Full Cylinders from Batch-2

\subsubsection{Compression Tests on Split-Bonded Cylinders}

Bonding of Split cylinders carried out as described in section 5.4.2 and are tested under axial compression as per testing configuration and wrapping scheme explained in the following sections.

\subsubsection{Compressive Strength of Non-Wrapped Split-Bonded Cylinders}

Non-wrapped split-bonded cylinders of batch-1 and batch- 2 were tested in axial compression. The failure load, average strength and stress are tabulated in Table 5.6. These values are compared to the values of non-wrapped full cylinders to evaluate the strength regained by the split bonded cylinders as compared to full cylinders. 
The average stress obtained by the non-wrapped split-bonded cylinders for batch-1 and batch-2 are $3.94 \mathrm{ksi}$ and $4.47 \mathrm{ksi}$ against $5.46 \mathrm{ksi}$ and $6.40 \mathrm{ksi}$ of non-wrapped full cylinders respectively.

Strength recovery ratio of Table 5.6 shows that the repair done by bonding split cylinders did not help in regaining full strength as that of non-wrapped full cylinders but there is a significant regain of $72 \%$ and $70 \%$ of strength for batch-1 and batch-2 respectively.

Table 5-6 Compressive Strength of Non-Wrapped Split-Bonded Cylinders

\begin{tabular}{|c|c|c|c|c|c|c|c|}
\hline \multirow[t]{2}{*}{ Batch } & \multirow[t]{2}{*}{ Description } & Split in & $\begin{array}{c}\text { Axial } \\
\text { Strength }\end{array}$ & $\begin{array}{c}\text { Avg. } \\
\text { Axial } \\
\text { Strength }\end{array}$ & $\begin{array}{l}\text { Axial } \\
\text { Stress }\end{array}$ & $\begin{array}{l}\text { Avg. } \\
\text { Axial } \\
\text { Stress } \\
\left(f_{\mathrm{coo}}{ }^{\prime}\right)\end{array}$ & $\begin{array}{c}\text { Strength } \\
\text { Recovery } \\
\text { Ratio }\end{array}$ \\
\hline & & Cylinder? & (kip) & (kip) & (ksi) & (ksi) & \\
\hline Batch 1 & $\begin{array}{c}\text { Control } \\
\text { Cylinders }\end{array}$ & $\mathrm{N}$ & - & 154.28 & - & 5.46 & - \\
\hline \multirow{3}{*}{ Batch 1} & \multirow{3}{*}{$\begin{array}{c}\text { Split- } \\
\text { Bonded }\end{array}$} & \multirow{3}{*}{$\mathrm{Y}$} & 105 & \multirow{3}{*}{111.33} & 3.71 & \multirow{3}{*}{3.94} & \multirow{3}{*}{0.72} \\
\hline & & & 109 & & 3.86 & & \\
\hline & & & 120 & & 4.24 & & \\
\hline Batch 2 & $\begin{array}{c}\text { Control } \\
\text { Cylinders }\end{array}$ & $\mathrm{N}$ & - & 181.00 & - & 6.40 & - \\
\hline \multirow{3}{*}{ Batch 2} & \multirow{3}{*}{$\begin{array}{c}\text { Split- } \\
\text { Bonded }\end{array}$} & \multirow{3}{*}{ Y } & 135 & \multirow{3}{*}{126.33} & 4.77 & \multirow{3}{*}{4.47} & \multirow{3}{*}{0.70} \\
\hline & & & 124 & & 4.39 & & \\
\hline & & & 120 & & 4.24 & & \\
\hline
\end{tabular}

Note: N-No; Y-Yes

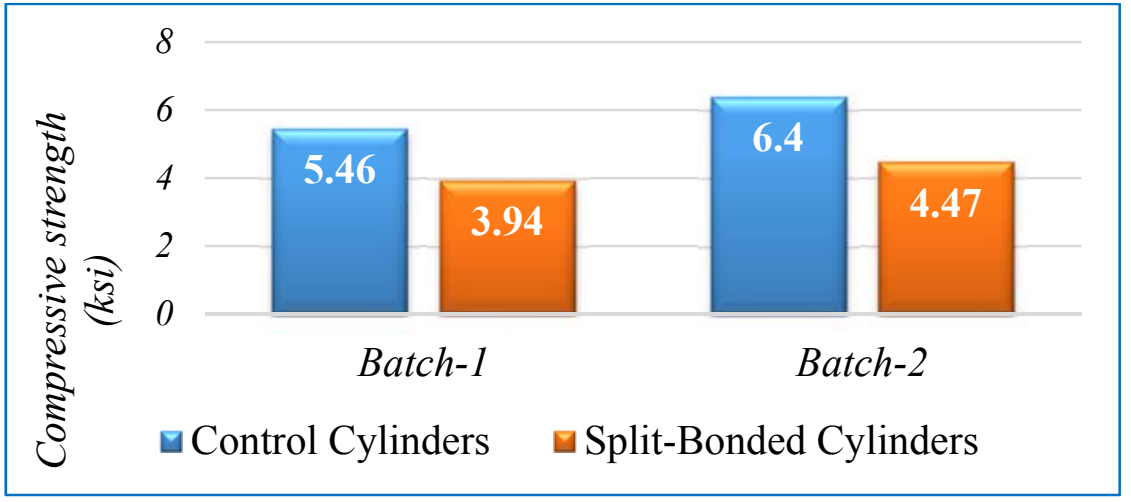

Figure 5-46 Average Axial Compressive Strength of Non-Wrapped Full and Split-Bonded Cylinders of Batch-1 and Batch-2 


\subsubsection{Compressive Strength of Wrapped Split-Bonded Cylinders}

Split-bonded cylinders were wrapped with two layers of HFRP pre-preg for batch-1 and of CFRP for batch-2. Wrapped split-bonded cylinders are tested under axial compression to determine the ultimate load. The test results for batch-1 and batch-2 are tabulated in Tables 5.7 and 5.8.

The tabulated results are compared to the values of two layer wrapped full cylinders of the respective batches to determine the strength difference between wrapped full cylinders and wrapped split-bonded cylinders.

Table 5-7 Compressive Strength of HFRP Pre-preg Wrapped Split-Bonded Cylinders of Batch-1

\begin{tabular}{|c|c|c|c|c|c|c|c|c|}
\hline \multirow{2}{*}{ FRP } & \multirow{2}{*}{$\begin{array}{l}\text { No. of } \\
\text { Layers }\end{array}$} & \multicolumn{2}{|c|}{ Split in } & \multirow{2}{*}{$\begin{array}{c}\begin{array}{c}\text { Axial } \\
\text { Strength }\end{array} \\
\text { (kip) }\end{array}$} & \multirow{2}{*}{$\begin{array}{c}\begin{array}{c}\text { Avg. } \\
\text { Axial } \\
\text { Strength }\end{array} \\
\text { (kip) }\end{array}$} & \multirow{2}{*}{\begin{tabular}{|c} 
Stress \\
$(\mathrm{ksi})$
\end{tabular}} & \multirow{2}{*}{$\begin{array}{l}\text { Avg. } \\
\text { Stress } \\
\text { (ksi) }\end{array}$} & \multirow{2}{*}{$\begin{array}{c}\text { Strength } \\
\text { Ratio }\end{array}$} \\
\hline & & Cylinder? & Wrap? & & & & & \\
\hline \multirow{7}{*}{ HFRP } & \multirow{3}{*}{2} & \multirow{3}{*}{$\mathrm{Y}$} & \multirow{3}{*}{$\mathrm{N}$} & 237 & \multirow{3}{*}{257} & 8.38 & \multirow{3}{*}{9.08} & \multirow{3}{*}{ - } \\
\hline & & & & 270 & & 9.55 & & \\
\hline & & & & 263 & & 9.30 & & \\
\hline & \multicolumn{8}{|c|}{ Comparison with non-wrapped full cylinders } \\
\hline & 0 & $\mathrm{~N}$ & - & - & 154 & - & 5.46 & - \\
\hline & 2 & $\mathrm{~N}$ & $\mathrm{~N}$ & - & 268 & - & 9.47 & 1.74 \\
\hline & 2 & $\mathrm{Y}$ & $\mathrm{N}$ & - & 257 & - & 9.08 & 1.66 \\
\hline
\end{tabular}

Note: N-No; Y-Yes

* The average stress obtained by the HFRP pre-preg wrapped split-bonded cylinders of batch-1 is 9.08 ksi vs 9.47 ksi of wrapped full cylinders.

* Strength recovery ratio of Table 5.7 shows that HFRP pre-preg wrapped split-bonded cylinders of batch-1 has a strength ratio of 1.66 vs 1.74 for HFRP wrapped full cylinders.

* The comparison shows that HFRP wrapped split-bonded cylinders has nearly the similar strength as HFRP wrapped full cylinders. 
Table 5-8 Compressive Strength of CFRP Wrapped Split-Bonded Cylinders of Batch-2

\begin{tabular}{|c|c|c|c|c|c|c|c|c|}
\hline \multirow{2}{*}{ FRP } & \multirow{2}{*}{$\begin{array}{l}\text { No. of } \\
\text { Layers }\end{array}$} & \multicolumn{2}{|c|}{ Split in } & \multirow{2}{*}{$\begin{array}{c}\begin{array}{c}\text { Axial } \\
\text { Strength }\end{array} \\
\text { (kip) }\end{array}$} & \multirow{2}{*}{$\begin{array}{c}\begin{array}{c}\text { Avg. } \\
\text { Axial } \\
\text { Strength }\end{array} \\
\text { (kip) }\end{array}$} & \multirow{2}{*}{\begin{tabular}{|r} 
Stress \\
(ksi)
\end{tabular}} & \multirow{2}{*}{$\begin{array}{l}\text { Avg. } \\
\text { Stress } \\
\text { (ksi) }\end{array}$} & \multirow{2}{*}{$\begin{array}{c}\text { Strength } \\
\text { Ratio }\end{array}$} \\
\hline & & Cylinder? & Wrap? & & & & & \\
\hline \multirow{7}{*}{ HFRP } & \multirow{3}{*}{2} & \multirow{3}{*}{ Y } & \multirow{3}{*}{$\mathrm{N}$} & $328 *$ & \multirow{3}{*}{412} & 11.60 & \multirow{3}{*}{14.55} & \multirow{3}{*}{ - } \\
\hline & & & & 423 & & 14.96 & & \\
\hline & & & & 400 & & 14.15 & & \\
\hline & \multicolumn{8}{|c|}{ Comparison with non-wrapped full cylinders } \\
\hline & 0 & $\mathrm{~N}$ & - & - & 181 & - & 6.40 & - \\
\hline & 2 & $\mathrm{~N}$ & $\mathrm{~N}$ & - & 437 & - & 15.46 & 2.41 \\
\hline & 2 & Y & $\mathrm{N}$ & - & 412 & - & 14.55 & 2.27 \\
\hline
\end{tabular}

Note: N-No; Y-Yes

* Outlier not considered in calculating average values

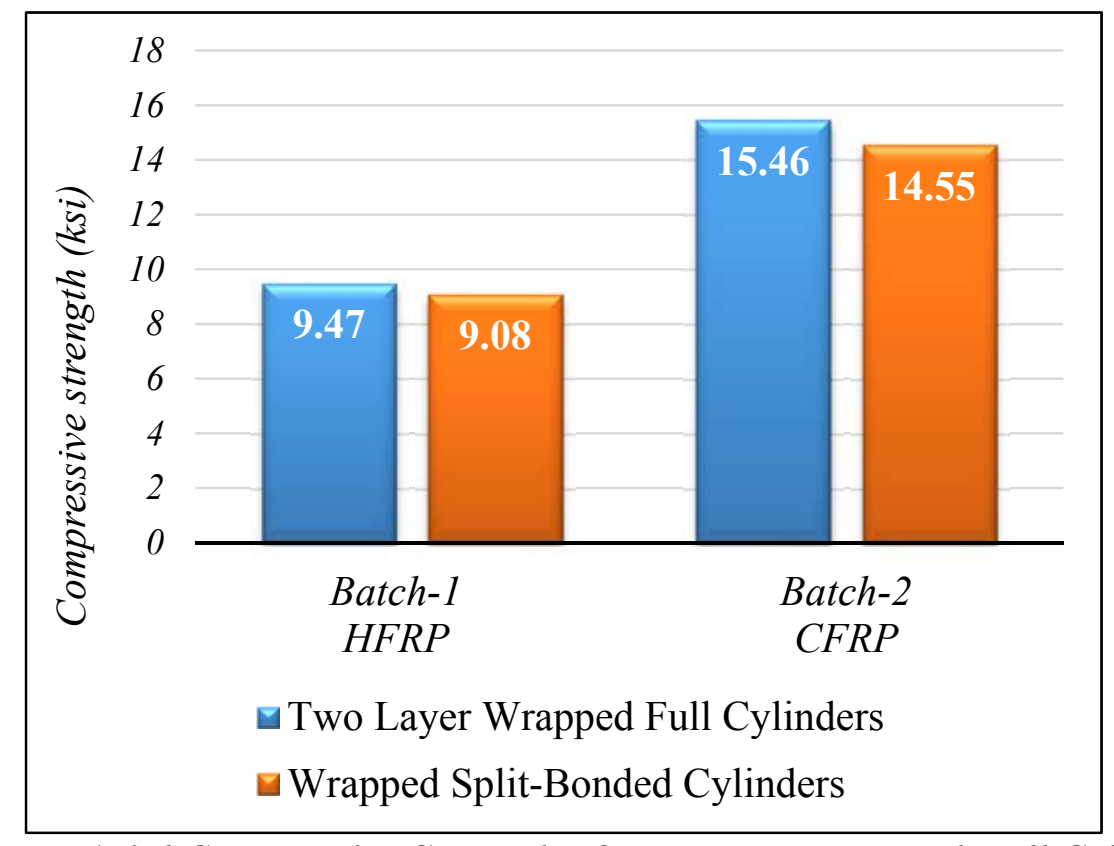

Figure 5-47 Average Axial Compressive Strength of Two Layer Wrapped Full Cylinders and Wrapped Split-Bonded Cylinders of Batch-1 and Batch-2

* The average stress obtained by the CFRP wrapped split-bonded cylinders of batch-2 is 14.55 ksi against 15.46 ksi of CFRP wrapped full cylinders. 
* Strength recovery ratio of Table 5.8 shows that CFRP wrapped split-bonded cylinders of batch-2 has strength ratio of 2.27 vs 2.41 for CFRP wrapped full cylinders.

* The comparison shows that CFRP wrapped split-bonded cylinders has nearly the similar strength as CFRP wrapped full cylinders.

\subsubsection{Stiffness/ Stress-Strain Curves of Split-Bonded Cylinders}

Cylinder specimens were tested under axial compression, load and strain data were collected through data acquisition system. Stress calculated from the obtained load data and plotted against strain data. The stress-strain curves plotted for the split-bonded cylinders are given in Fig. 48, 49.

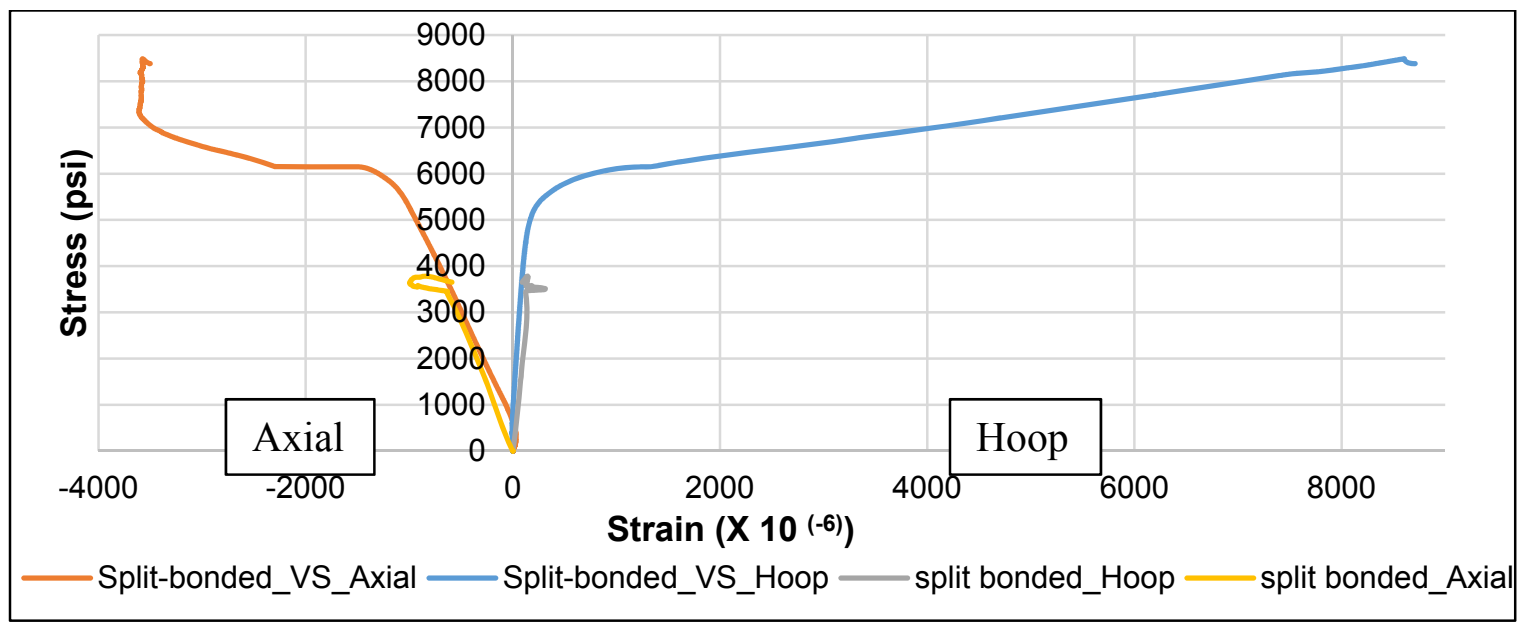

Figure 5-48 Comparison between Non-Wrapped and 2 layer HFRP Pre-preg Wrapped SplitBonded Cylinders of Batch-1

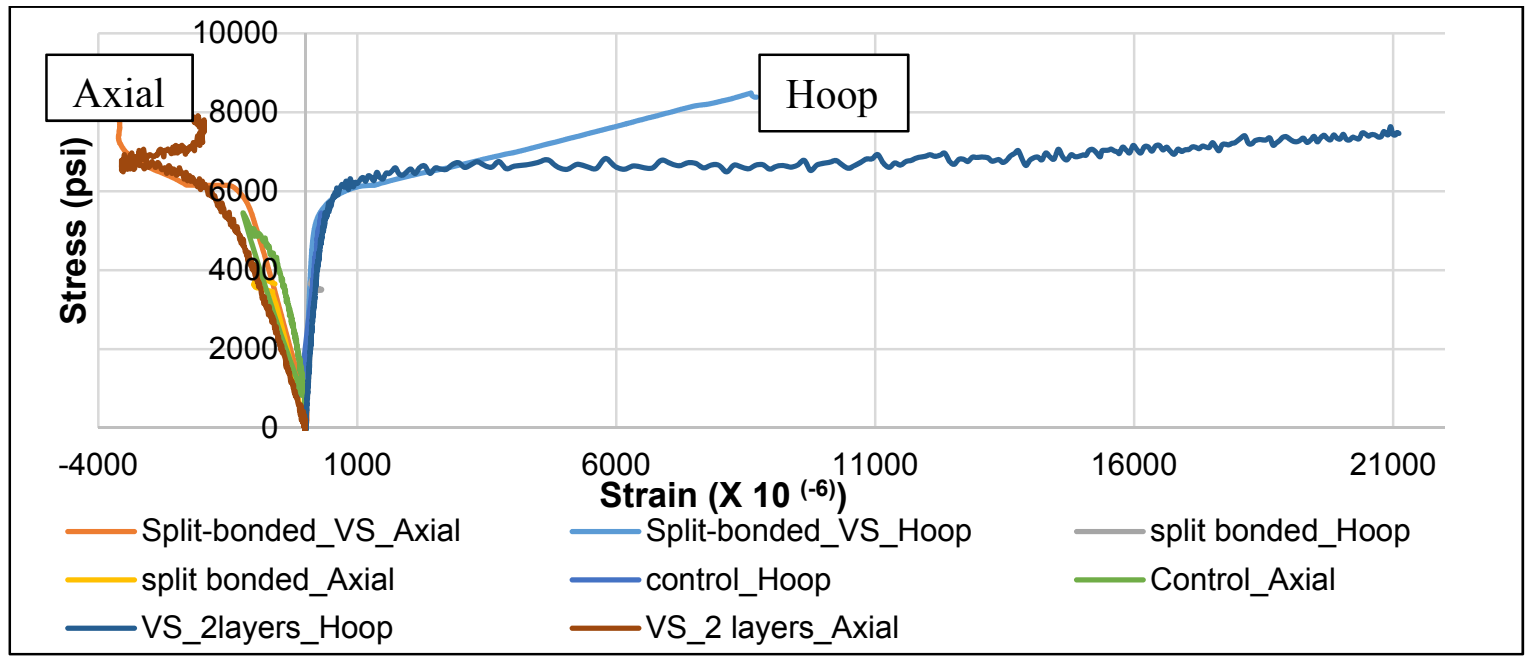

Figure 5-49 Comparison between Full and Split-Bonded Cylinders of Batch-1 


\subsubsection{Failure Modes of Split-Bonded Cylinders}

The failure of non-wrapped split-bonded cylinders from batch-1 and batch-2 was brittle. The failure modes varied from well-formed cone on one end to columnar vertical cracking through both ends with no well-formed cones which are Type 2 and Type 3 failure modes according to ASTM C39. Also seen that the cracks formed were away from the bonded line for 5 of 6 nonwrapped split-bonded cylinders tested from two batches.

The failure of split-bonded cylinders wrapped with two layers of HFRP Pre-preg and CFRP-epoxy system was similar to the failure mode as explained for wrapped full cylinders in section 5.6.1.6. The failure modes of all the split-bonded cylinders are shown below in Fig 5.50 to 5.53
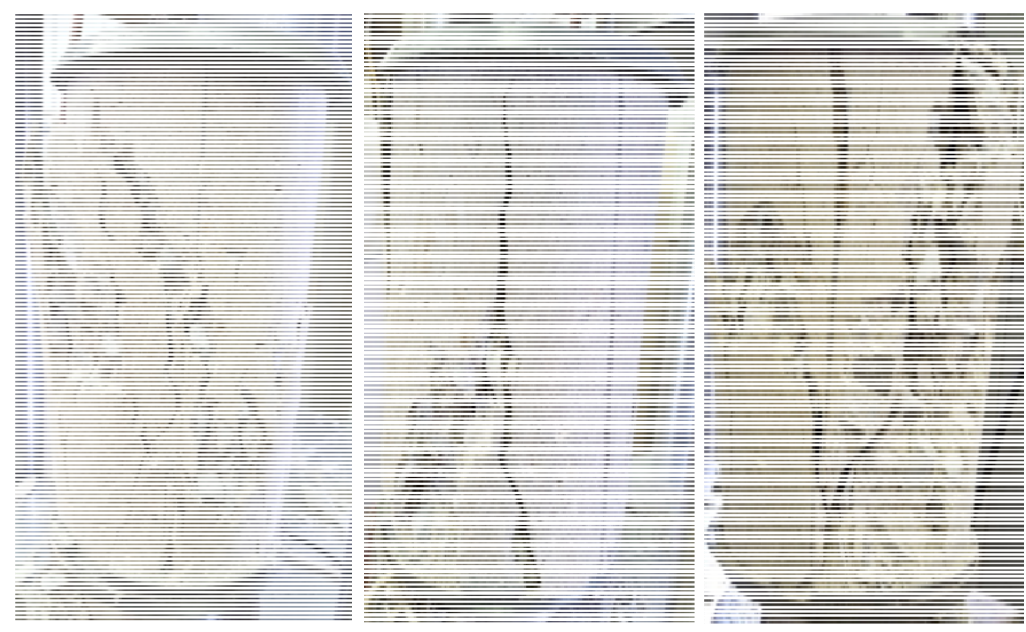

Figure 5-50 Split-Bonded Cylinders of Batch-1

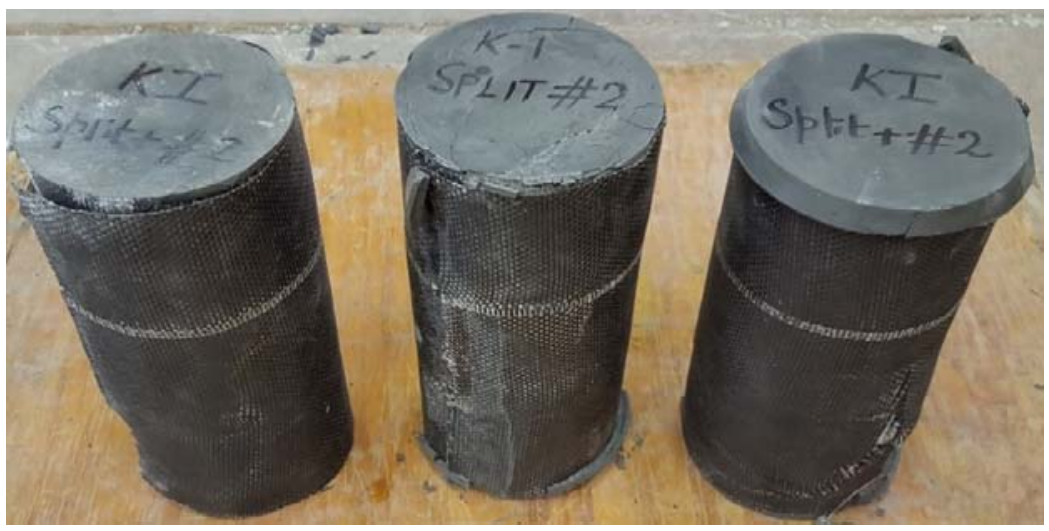

Figure 5-51 Two Layer HFRP Pre-preg Wrapped Split-Bonded Cylinders of Batch-1 

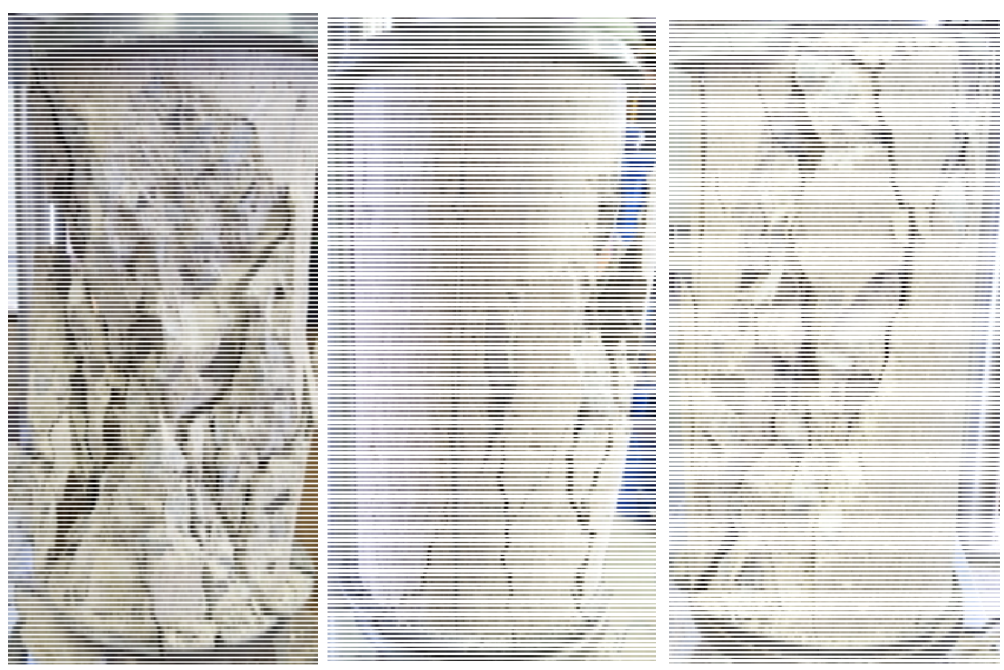

Figure 5-52 Split-Bonded Cylinders of Batch-2
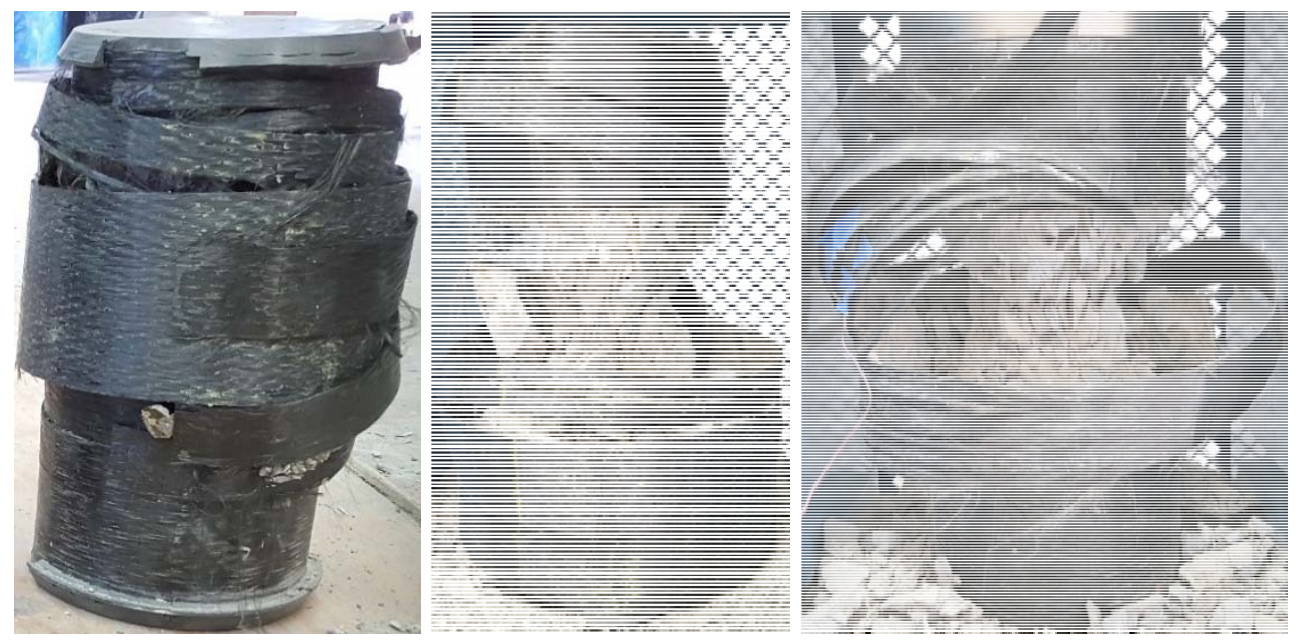

Figure 5-53 Two Layer CFRP Wrapped Split-Bonded Cylinders of Batch-2

\subsection{Summary}

In this chapter, the test results and analysis of the tests conducted on the concrete cylinders confined with FRP wraps were discussed. The effect of confinement was presented in the form of tables and graphs. Variation in strength, stiffness and ductility due to number of layers of wrap on full cylinders and split-bonded cylinders have been provided and the failure modes of the specimens were described with good pictures. The results obtained to determine each parameter of confinement have been analyzed and presented. 


\section{RC BEAMS BENDING TESTS}

\subsection{Introduction}

This chapter consists of flexural tests conducted on plain and externally strengthened reinforced concrete beams with FRP. The results are presented in tables and graphs. All specimens were externally strengthened on tension side manually with FRP. Comparison of load, strain, etc. between plain and strengthened specimens has been discussed in the following sections. The test results were analyzed to evaluate the efficacy of the FRP strengthening.

\subsection{Test Description}

Twelve reinforced concrete beams of size 6"x 15" x 120" were cast in two batches with two different concrete strength mixes, six in each batch. They were reinforced internally with No.3 steel rebar which includes two compression rebar, two tension rebar and stirrups (shear rebar) at 5-inch center to center distance. After the specimens cured for 28 days, they were tested in fourpoint bending under cyclic loading as per following beam strengthening schemes.

i. External strengthening of beams with CFRP-Epoxy system.

ii. External strengthening of beams with HFRP Pre-preg.

\subsection{Equipment and Test Set-up}

\subsubsection{Test Set-up}

The beams to be tested under four-point bending were placed on supports placed at a distance of $9 \mathrm{ft}$ having a 6-inch overhang on both the ends with a hydraulic actuator being positioned at the center. An I-beam was placed over two rollers and plate supports located on top and at the middle third span of the beam. Data acquisition systems were arranged as discussed in section 6.3.2. Loads 
were applied manually using hydraulic jack. Figure 6.1 shows the test set-up for four-point bending test on beam.

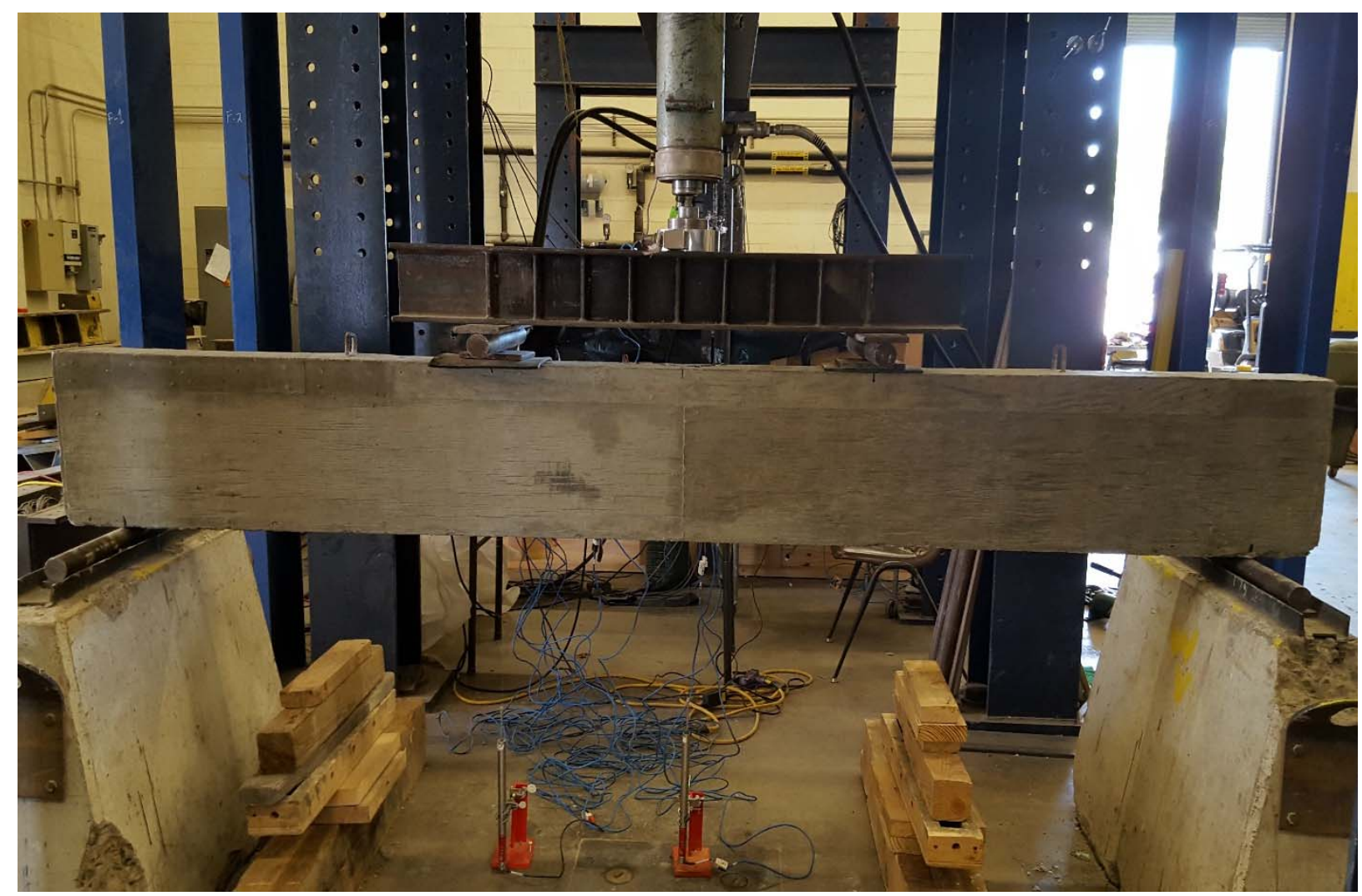

Figure 6-1 Test Set-up for Four Point Bending Tests on RC Beams

\subsubsection{Strain Gages and Data Acquisition}

To record and collect the test data, data acquisition system 7000 was used. It has strain gage cards and high-level cards with a capacity of fifty-six channels. For beam bending testing, strain gage cards are used to record data from strain gages those installed at the mid-span of the beam on concrete in compression \& tension, compression, tension \& shear rebars, externally strengthened FRP on tension side of the beam. STRAIN SMART software (Fig 4.3(b)) was used to interpret the acquired data by exporting the recorded readings in to excel files. Strain gages used for data recording were manufactured by Vishay Micro-measurements with a resistance of $350 \mathrm{ohm}$ (Fig 4.3(c)). M200-bond from Vishay Micro-measurements was used to attach them on to the surface of CFRP wraps and AE10-bond for HFRP Pre-preg wraps. 
A calibrated load cell of 50-kip capacity was connected to the strain gage card of the data acquisition system for obtaining continuous load data. Load cell was placed right below the hydraulic actuator to obtain the load data as shown in Figure 6.1. LVDTs were also connected at the mid span and middle third span of the beam to obtain load deflection curves.

\subsection{Specimen Preparation}

\subsubsection{Reinforced Concrete Beams:}

Reinforced concrete beam specimens were cast in wooden formwork. Rebar cages were prepared with bent rebar (stirrups) over the compression and tension rebar throughout the span of the beam. Wooden Formwork surfaces were cleaned and greased with oil. Prepared rebar cages (Fig 6.2) were positioned in the oiled formwork by placing the chairs for appropriate spacing (Fig 6.3). After positioning the rebar alignment and clear spacing, concrete pouring was taken place. Vibrator was used for compaction of concrete and to release entrapped air. Surface was smoothened using a levelling tool and kept them aside for 2 days. They were then demolded and cured with wet burlaps for 28 days.

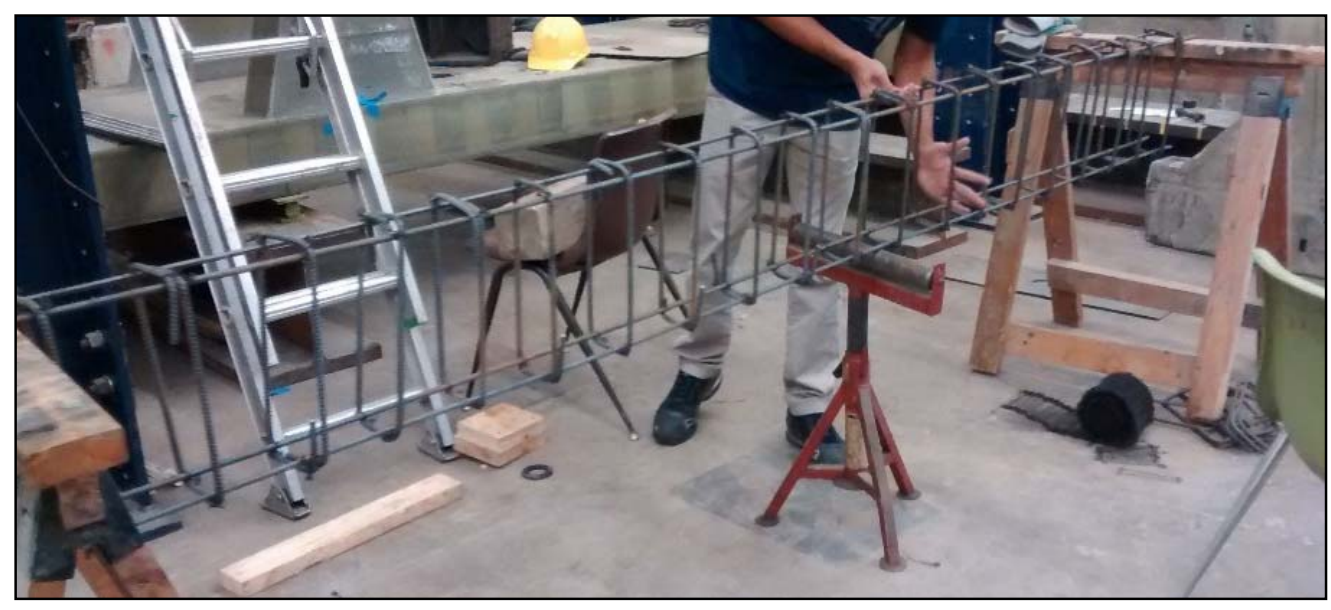

Figure 6-2 Making of Steel Rebar Cage 


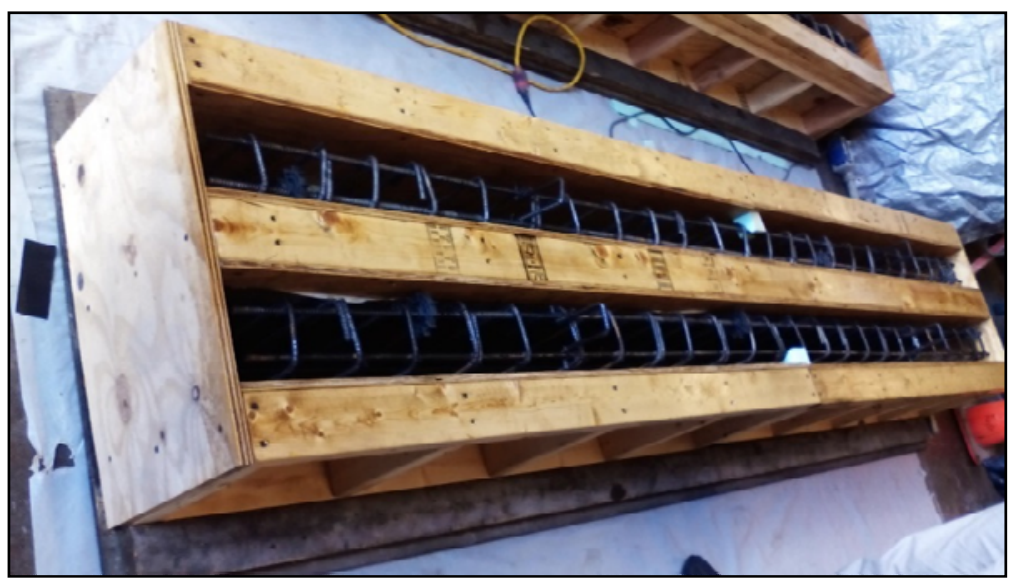

Figure 6-3 Wooden Formwork with Rebar Cage in Position

\subsubsection{FRP Wrapping}

After curing, beams were wrapped on tension side with FRP fabrics. Wrapping procedure is similar to that being explained in section 5.4.3 except that they are applied only on tension face of the beam as shown in Fig 6.4 .
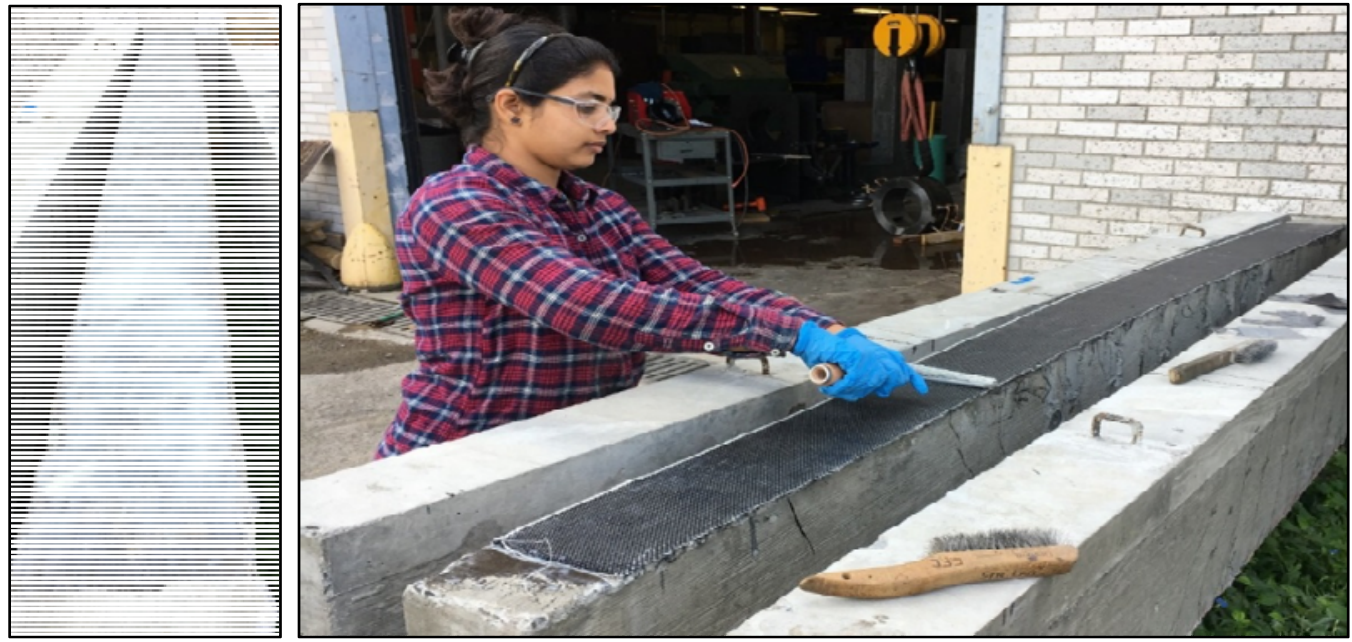

Figure 6-4 Application of Primer (Left Side), Beam Wrapping with FRP (Right Side)

Prior to wrapping, beams were prepared as explained below:

- Pre-cracking of beams: Beams were loaded until first crack and then wrapped with FRP. Pre-cracking was done for all beams except for control beams. 
- Damaging and repairing concrete (Fig 6.5): For some of the beams, concrete on tension side was damaged using jack hammer or chiseling and repaired with Sika Quick VOH repair mortar. These repaired beams were loaded up to cracking load and then wrapped with FRP wraps.
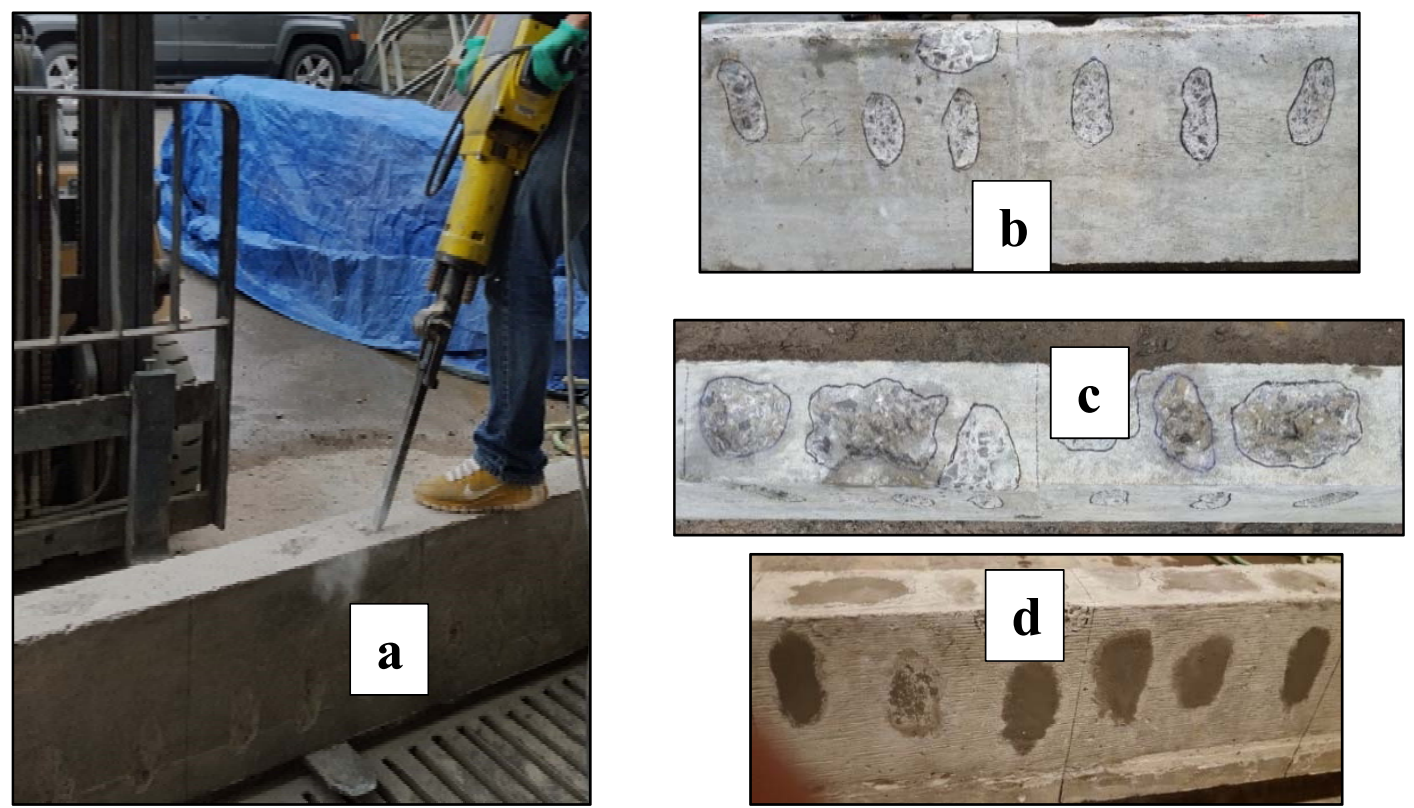

Figure 6-5 (a) Jack Hammer; (b) Side View; (c) Top View; (d) Mortar Repair

Beam configurations were adopted as described below:

Control Beams: The control beams from batch-1 and batch-2 were loaded until failure without any wrapping.

\section{CFRP Wrapped Beams:}

Batch-1: Beam-1 and Beam-2 were wrapped with 2 layers of CFRP. Prior to wrapping they were damaged on tension side. Beam-1 was damaged only on tension face in mid-span whereas beam2 was damaged on tension face as well as on sides of the beam in middle length zone. Repaired damaged portion, pre-cracked, wrapped and loaded until failure.

Batch-2: Beam-1 was wrapped with 2 layers of CFRP and Beam-2 was wrapped with 3 layers of CFRP. 2 layered beam was pre-cracked, wrapped, loaded until crack, cracks sealed using crack 
injection procedure and loaded until failure. 3 layered beam was only pre-cracked, wrapped and loaded until failure.

\section{HFRP Wrapped Beams:}

Batch-1: Beam-1 and Beam-2 were wrapped with 2 layers of HFRP. Beam-1 was damaged on tension face in middle length zone and beam- 2 on tension face as well as on sides throughout the length of the beam. Repaired damaged portion, pre-cracked, wrapped and loaded until failure.

Batch-2: Beam-1 was wrapped with 2 layers of HFRP and Beam-2 was wrapped with 3 layers of HFRP. 2 layered beam was pre-cracked, wrapped, loaded until crack, crack sealed using crack injection procedure and loaded until failure. 3 layered beam was only pre-cracked, wrapped and loaded until failure.

\subsection{Test Procedure}

After setting up beam as described in section 6.3, four-point loading was applied on the beam in three loading and unloading cycles until the failure of the beam.

\subsection{Test Results and Analysis}

Experimental results of load and bending moments for beams tested under four-point bending are compared with values obtained from theoretical calculations as per theory of bending for reinforced concrete beams. The theoretical calculations are presented in Appendix E. Experimental to theoretical ratios ranged from 1 to 1.07 for control beams, 0.91 to 1.04 for CFRP wrapped beams, and 0.76 to 0.98 for HFRP wrapped beams. These comparisons are shown in Tables 6.1 to 6.5 . 
Effective debonding strains $\left(\varepsilon_{f d}\right)$ in FRP were calculated as per ACI $440.2 \mathrm{R}-08$ and are also presented in Tables 6.1 to 6.5. The range of $\varepsilon_{f d}$ seen from theoretical calculations lies between 0.0062 to 0.0078 for CFRP and 0.0096 to 0.0118 for HFRP whereas the actual debonding strains obtained from experimental data are in a range of 0.0032 to 0.0041 for CFRP and 0.0067 to 0.0102 for HFRP. Hence, $60 \%$ of effective debonding strains were considered in theoretical calculations.

\subsubsection{Control Beams}

One reinforced concrete beam from batch-1 and two reinforced concrete beams from batch- 2 were tested under four point bending and the results are tabulated in Table 6.1. Ultimate stress instead of yield stress was considered for comparing experimental to theoretical calculations according to the literature review and in reference to the stress-strain curves of steel rebar that is discussed in Appendix A. 
Table 6-1 Four Point Bending on Control Beams from Batch-1 and Batch-2

\begin{tabular}{|c|c|c|c|c|c|c|c|c|}
\hline \multirow{3}{*}{ Batch } & \multirow{3}{*}{ Beam } & \multicolumn{2}{|c|}{ Stress in Steel Rebar } & $\begin{array}{l}\text { Max. } \\
\text { Load }\end{array}$ & Max. Load & $\begin{array}{c}\text { Max. } \\
\text { Moment }\end{array}$ & $\begin{array}{c}\text { Max. } \\
\text { Moment }\end{array}$ & \multirow{3}{*}{$\begin{array}{l}\text { Exptl./ } \\
\text { Theor. } \\
\text { Ratio }\end{array}$} \\
\hline & & $f_{y}$ & $f_{u}^{*}$ & (Exptl.) & (Theor.) & (Exptl.) & (Theor.) & \\
\hline & & (ksi) & (ksi) & (kip) & (kip) & (k-ft) & (k-ft) & \\
\hline $\begin{array}{l}\text { Batch-1 } \\
\text { (5.46 ksi) }\end{array}$ & $\begin{array}{c}\text { Control } \\
\text { Beam }\end{array}$ & \multirow{3}{*}{60} & 85 & 12.54 & 13.34 & 18.81 & 20.01 & 0.94 \\
\hline \multirow{2}{*}{$\begin{array}{l}\text { Batch-2 } \\
(6.40 \mathrm{ksi})\end{array}$} & $\begin{array}{l}\text { Control } \\
\text { Beam-1 }\end{array}$ & & \multirow{2}{*}{85} & 16.15 & 13.35 & 24.23 & 20.03 & 1.21 \\
\hline & $\begin{array}{l}\text { Control } \\
\text { Beam-2 }\end{array}$ & & & 15.07 & 13.35 & 22.61 & 20.03 & 1.13 \\
\hline
\end{tabular}

*Values taken from actual stress-strain curves of steel bar (Refer Appendix A) and are used for theoretical calculations

\subsubsection{External strengthening of beams with CFRP-Epoxy System}

Two reinforced concrete beams from each batch were wrapped on tension side using CFRP-epoxy system. For batch-1, beams were damaged one in in middle third zone (tension face and side of the beam towards tension zone) and other in tension zone throughout the span (only on tension face) as explained in section 6.4.2 and wrapped with two layers each. For batch-2, one beam with two layers and 
other with three layers of the wrapping system. Test results on testing them under four point bending are tabulated in Table 6.2 and Table 6.3. The results are compared with those of the control beams.

Table 6-2 Four Point Bending on Externally Strengthened Beams with CFRP-Epoxy System from Batch-1 (5.46 ksi)

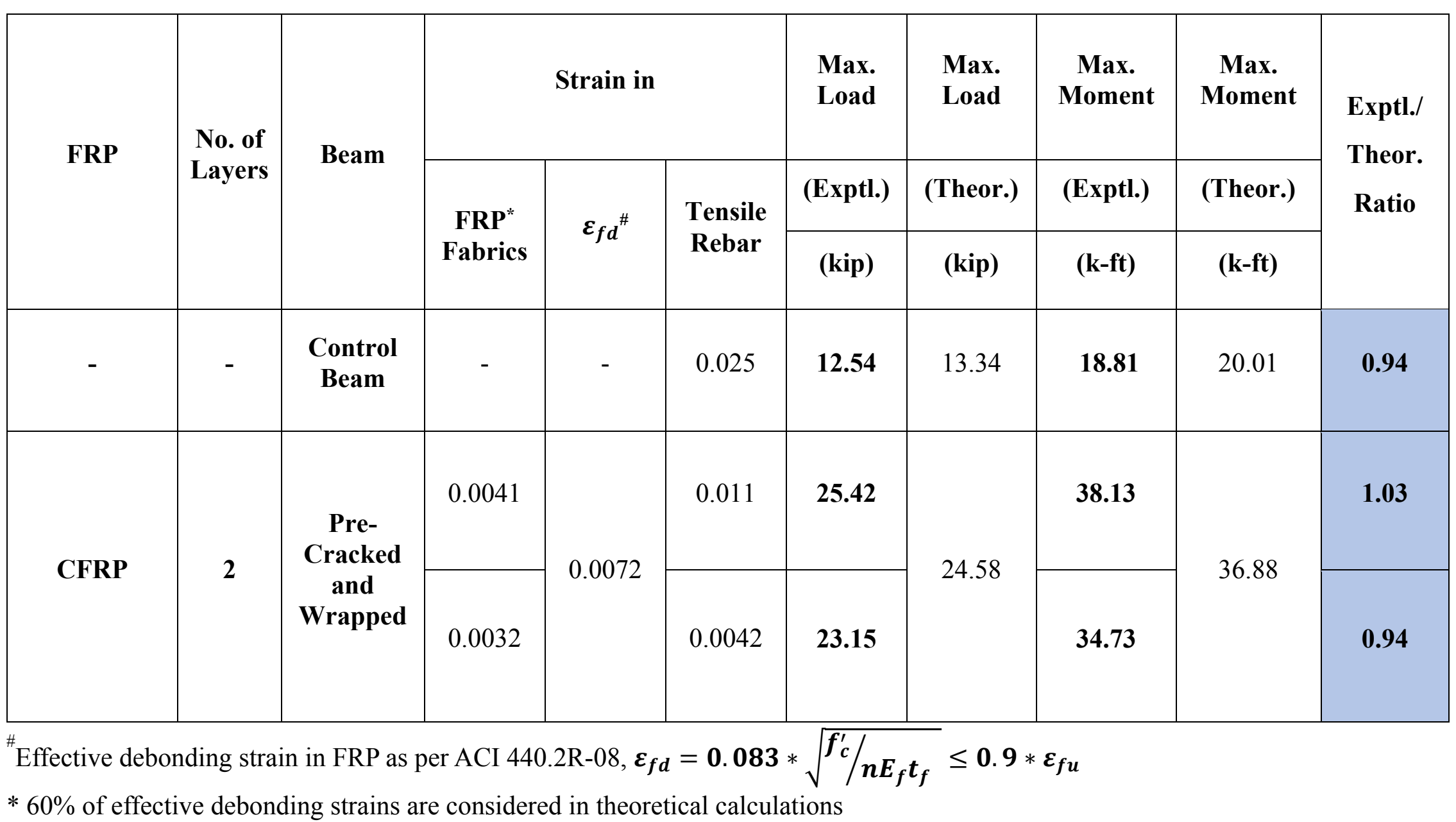


Table 6-3 Four Point Bending on Externally Strengthened Beams with CFRP-Epoxy System from Batch-2 (6.4 ksi)

\begin{tabular}{|c|c|c|c|c|c|c|c|c|c|c|}
\hline \multirow{3}{*}{ FRP } & \multirow{3}{*}{$\begin{array}{c}\text { No. of } \\
\text { Layers }\end{array}$} & \multirow{3}{*}{ Beam } & \multicolumn{3}{|c|}{ Failure Strain in } & \multirow{2}{*}{$\begin{array}{c}\text { Max. } \\
\text { Load }\end{array}$} & \multirow{2}{*}{$\begin{array}{c}\text { Max. } \\
\text { Load }\end{array}$} & \multirow{2}{*}{$\begin{array}{c}\text { Max. } \\
\text { Moment }\end{array}$} & \multirow{2}{*}{$\begin{array}{c}\text { Max. } \\
\text { Moment }\end{array}$} & \multirow{3}{*}{$\begin{array}{c}\text { Exptl./ } \\
\text { Theor. } \\
\text { Ratio }\end{array}$} \\
\hline & & & \multirow{2}{*}{$\begin{array}{c}\text { FRP* } \\
\text { Fabrics }\end{array}$} & \multirow{2}{*}{$\varepsilon_{f d}{ }^{\#}$} & \multirow{2}{*}{$\begin{array}{l}\text { Tensile } \\
\text { Rebar }\end{array}$} & & & & & \\
\hline & & & & & & (kip) & (kip) & (k-ft) & $(k-f t)$ & \\
\hline \multirow{2}{*}{ - } & \multirow{2}{*}{ - } & $\begin{array}{l}\text { Control } \\
\text { Beam-1 }\end{array}$ & - & - & 0.025 & 16.15 & 13.35 & 24 & 20.03 & 1.21 \\
\hline & & $\begin{array}{l}\text { Control } \\
\text { Beam-2 }\end{array}$ & - & - & 0.020 & 15.07 & 13.35 & 22.5 & 20.03 & 1.13 \\
\hline \multirow{2}{*}{ CFRP } & 2 & \multirow{2}{*}{$\begin{array}{c}\text { Pre- } \\
\text { Cracked } \\
\text { and } \\
\text { Wrapped }\end{array}$} & $0.0047^{+}$ & 0.0078 & 0.017 & 26.98 & 25.90 & 40.47 & 38.85 & 1.04 \\
\hline & 3 & & 0.0035 & 0.0062 & 0.011 & 27.23 & 29.98 & 40.85 & 44.98 & 0.91 \\
\hline
\end{tabular}

${ }^{\#}$ Effective debonding strain in FRP as per ACI 440.2R-08, $\boldsymbol{\varepsilon}_{\boldsymbol{f} \boldsymbol{d}}=\mathbf{0 . 0 8 3} * \sqrt{\boldsymbol{f}_{\boldsymbol{c}}^{\prime} / \boldsymbol{n} \boldsymbol{E}_{\boldsymbol{f}} \boldsymbol{t}_{\boldsymbol{f}}} \leq \mathbf{0 . 9} * \boldsymbol{\varepsilon}_{\boldsymbol{f} \boldsymbol{u}}$

* $60 \%$ of effective debonding strains are considered in theoretical calculations

${ }^{+}$Gage Malfunction 


\subsubsection{External strengthening of beams with HFRP Pre-preg}

Two reinforced concrete beams from each batch were wrapped on tension side with HFRP Pre-preg system. For batch-1, beams were damaged one in in middle third zone (only on tension face) and other in tension zone (tension face and side of the beam towards tension zone) throughout the span as explained in section 6.4.2 and wrapped with two layers of HFRP pre-preg each. For batch-2, one beam was wrapped with two layers and other with three layers of HFRP pre-preg. Wrapped beams were tested under four point bending and the test results are tabulated in Table 6.4 and Table 6.5. The results are compared with those of the corresponding control beams.

Table 6-4 Four Point Bending on Externally Strengthened Beams with HFRP Pre-preg System from Batch-1 (5.46 ksi)

\begin{tabular}{|c|c|c|c|c|c|c|c|c|c|c|}
\hline \multirow{3}{*}{ FRP } & \multirow{3}{*}{$\begin{array}{l}\text { No. of } \\
\text { Layers }\end{array}$} & \multirow{3}{*}{ Beam } & \multicolumn{3}{|c|}{ Failure Strain in } & \multirow{2}{*}{$\begin{array}{l}\text { Max. } \\
\text { Load }\end{array}$} & \multirow{2}{*}{$\begin{array}{l}\text { Max. } \\
\text { Load } \\
\text { (Theor.) }\end{array}$} & \multirow{2}{*}{$\begin{array}{c}\begin{array}{c}\text { Max. } \\
\text { Moment }\end{array} \\
\text { (Exptl.) }\end{array}$} & \multirow{2}{*}{$\begin{array}{c}\text { Max. } \\
\text { Moment }\end{array}$} & \multirow{3}{*}{$\begin{array}{l}\text { Exptl./ } \\
\text { Theor. } \\
\text { Ratio }\end{array}$} \\
\hline & & & \multirow{2}{*}{$\begin{array}{c}\text { FRP }^{*} \\
\text { Fabrics }\end{array}$} & \multirow{2}{*}{$\varepsilon_{f d}{ }^{\#}$} & \multirow{2}{*}{\begin{tabular}{|l|} 
Tensile \\
Rebar
\end{tabular}} & & & & & \\
\hline & & & & & & (kip) & (kip) & (k-ft) & (k-ft) & \\
\hline- & - & $\begin{array}{c}\text { Control } \\
\text { Beam }\end{array}$ & - & - & 0.025 & 12.54 & 13.34 & 18.81 & 20.01 & 0.94 \\
\hline \multirow{2}{*}{ HFRP } & \multirow{2}{*}{2} & \multirow{2}{*}{$\begin{array}{c}\text { Pre-Cracked } \\
\text { and } \\
\text { Wrapped }\end{array}$} & 0.0102 & \multirow{2}{*}{0.0109} & 0.016 & 19.25 & 19.56 & 28.88 & 29.35 & 0.98 \\
\hline & & & 0.0067 & & 0.019 & 16.15 & 19.40 & 24.23 & 29.09 & 0.83 \\
\hline
\end{tabular}

${ }^{\#}$ Effective debonding strain in FRP as per ACI 440.2R-08, $\boldsymbol{\varepsilon}_{\boldsymbol{f d}}=\mathbf{0 . 0 8 3} * \sqrt{\boldsymbol{f}_{\boldsymbol{c}}^{\prime} / \boldsymbol{n} \boldsymbol{E}_{\boldsymbol{f}} \boldsymbol{t}_{\boldsymbol{f}}} \leq \mathbf{0 . 9} * \boldsymbol{\varepsilon}_{\boldsymbol{f} \boldsymbol{u}}$

* $60 \%$ of effective debonding strains are considered in theoretical calculations 
Table 6-5 Four Point Bending on Externally Strengthened Beams with HFRP Pre-preg System from Batch-2 (6.4 ksi)

\begin{tabular}{|c|c|c|c|c|c|c|c|c|c|c|}
\hline \multirow{3}{*}{ FRP } & \multirow{3}{*}{$\begin{array}{c}\text { No. of } \\
\text { Layers }\end{array}$} & \multirow{3}{*}{ Beam } & \multicolumn{3}{|c|}{ Failure Strain in } & \multirow{2}{*}{$\begin{array}{l}\text { Max. } \\
\text { Load } \\
\text { (Exptl.) }\end{array}$} & \multirow{2}{*}{$\begin{array}{l}\text { Max. } \\
\text { Load } \\
\text { (Theor.) }\end{array}$} & \multirow{2}{*}{$\begin{array}{c}\text { Max. } \\
\text { Moment } \\
\text { (Exptl.) }\end{array}$} & \multirow{2}{*}{$\begin{array}{c}\begin{array}{c}\text { Max. } \\
\text { Moment }\end{array} \\
\text { (Theor.) }\end{array}$} & \multirow{3}{*}{$\begin{array}{l}\text { Exptl./ } \\
\text { Theor. } \\
\text { Ratio }\end{array}$} \\
\hline & & & \multirow{2}{*}{$\begin{array}{c}\text { FRP* } \\
\text { Fabrics }\end{array}$} & \multirow[t]{2}{*}{$\varepsilon_{f d}{ }^{\#}$} & \multirow{2}{*}{$\begin{array}{l}\text { Tensile } \\
\text { Rebar }\end{array}$} & & & & & \\
\hline & & & & & & (kip) & (kip) & (k-ft) & (k-ft) & \\
\hline \multirow[t]{2}{*}{ - } & \multirow[t]{2}{*}{ - } & $\begin{array}{l}\text { Control } \\
\text { Beam-1 }\end{array}$ & - & - & 0.025 & 16.15 & 13.35 & 24 & 20.03 & 1.21 \\
\hline & & $\begin{array}{l}\text { Control } \\
\text { Beam-2 }\end{array}$ & - & - & 0.020 & 15.07 & 13.35 & 22.5 & 20.03 & 1.13 \\
\hline \multirow[b]{2}{*}{ HFRP } & 2 & \multirow{2}{*}{$\begin{array}{c}\text { Pre-Cracked } \\
\text { and } \\
\text { Wrapped }\end{array}$} & 0.0082 & 0.0118 & 0.0127 & 19.99 & 20.44 & 29.99 & 30.66 & 0.98 \\
\hline & 3 & & 0.0069 & 0.0096 & 0.0141 & 17.36 & 22.86 & 26.04 & 34.28 & 0.76 \\
\hline
\end{tabular}

\# Effective debonding strain in FRP as per ACI 440.2R-08, $\boldsymbol{\varepsilon}_{\boldsymbol{f d}}=\mathbf{0 . 0 8 3} * \sqrt{\boldsymbol{f}_{\boldsymbol{c}}^{\prime} / \boldsymbol{n} \boldsymbol{E}_{\boldsymbol{f}} \boldsymbol{t}_{\boldsymbol{f}}} \leq \mathbf{0 . 9} * \boldsymbol{\varepsilon}_{\boldsymbol{f} \boldsymbol{u}}$

* $60 \%$ of effective debonding strains are considered in theoretical calculations

From the results, it can be inferred that the fabrics with lower bond strengths that has been observed at pull-off could exhibit similar failure pattern in flexure. 


\subsubsection{Load Deflection Curves}

Typical Load deflection curve plotted for all the tests conducted on beams is presented below in Fig 6.6.

Note: Debonding of the HFRP and subsequent drop from the concrete surface resulted in increase in deflection even after failure load for HFRP wrapped beams.

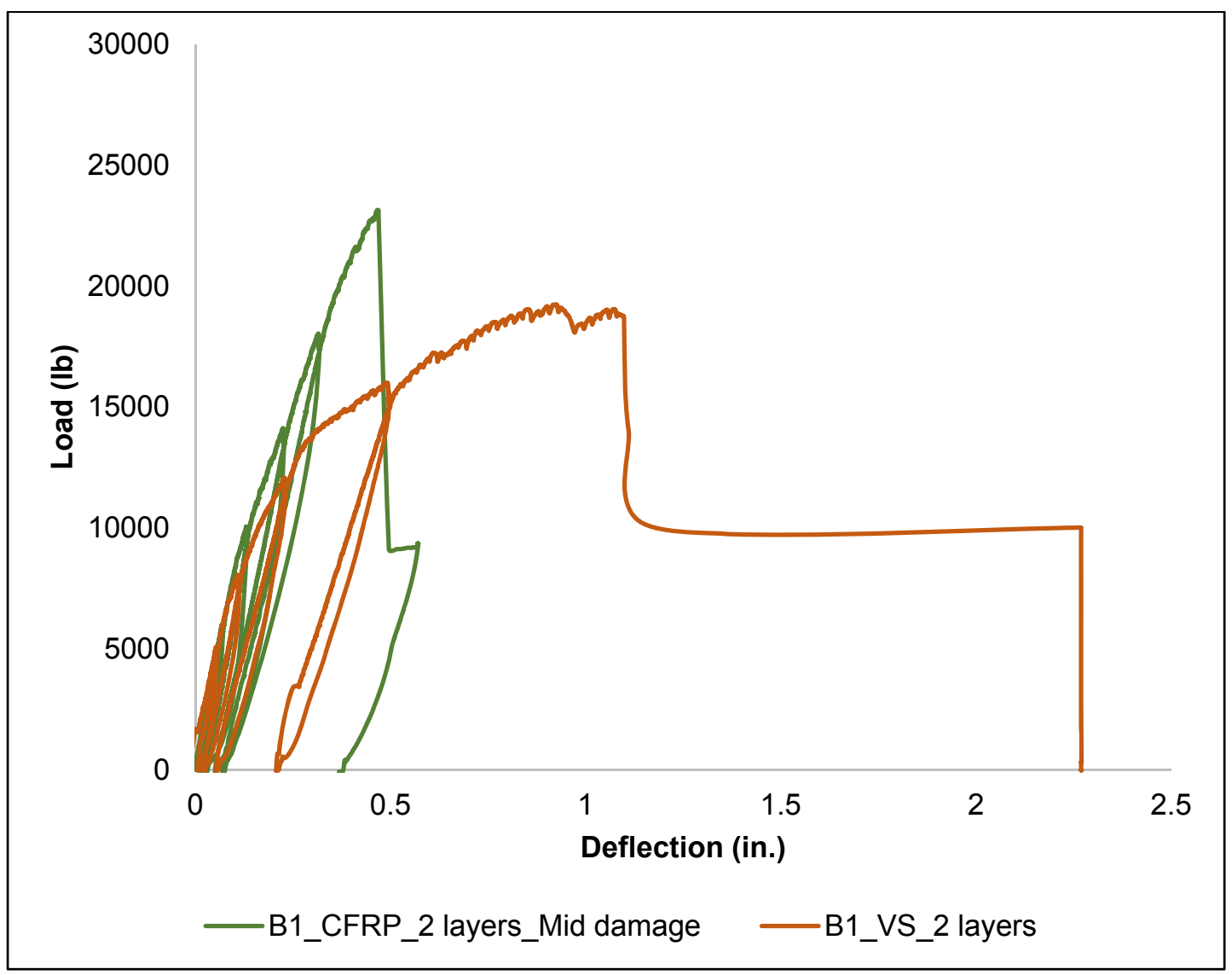

Figure 6-6 Load-Deflection Curves for CFRP and HFRP Wrapped Beams of Batch-1 


\subsubsection{Failure Modes}

Control Beams: The control beams from batch-1 and batch-2 were observed to be failed in flexure. The failure modes are shown in Fig 6.7 to 6.9. Cracking load was found at $6.2 \mathrm{kip}$ for batch-1 and 7.5kip for batch-2. Ultimate load of 12.54kip for batch-1 and 15kip, 16.15kip for batch-2. The values are within $121 \%$ of the theoretical values.

\section{CFRP Wrapped Beams:}

Batch-1: Beam-1 and Beam-2 were wrapped with 2 layers of CFRP. Prior to wrapping they were damaged on tension side as described in section 6.4.2. Maximum load taken by beams wrapped with 2 layers of CFRP were 25.42kip and 23.15kip for beam-1 and beam-2 respectively. Cracking observed at 6kip load. Beam-1 with damage only on tension face has more area under loaddeflection curve than beam-2 with damage on tension face as well as on sides in middle length zone.

Debonding failure along with concrete cover separation was observed at a strain level of 0.0041 and 0.0032 for beam-1 and beam-2 respectively. Due to debonding, FRP contribution was not utilized completely. This can be observed by comparing the CFRP strain at failure of beam with the ultimate strain in CFRP i.e., 0.012 that obtained from the tensile tests on CFRP coupons. The experimental values for load and moment were within $90 \%$ of theoretical values calculated using debonding strains. The failure modes are shown in Fig 6.10 and 6.11.

Batch-2: Maximum load taken by beams wrapped with 2 layers and 3 layers of CFRP were 26.98kip and 27.23kip respectively. Cracking observed at 8kip load for 2 layered beam and at 9kip for 3 layered beam. 2 layered beam was pre-cracked, wrapped, loaded until crack and then crack 
sealed before loading and as a result of the crack seal, pick up in stiffness was observed until 3.5kip and then the stiffness dropped suddenly at this load point (Appendix F).

Debonding failure along with concrete cover separation was observed at a strain level of 0.0047 and 0.0035 for 2 layered and 3 layered respectively. Due to debonding, FRP contribution was not utilized completely. This can be observed by comparing the CFRP strain at failure of beam with the ultimate strain in CFRP i.e., 0.012 that obtained from the tensile tests on CFRP coupons. The experimental values for load and moment were within $90 \%$ of theoretical values calculated using debonding strains. The failure modes are shown in Fig 6.12 and 6.13 .

\section{HFRP Wrapped Beams:}

Batch-1: Beam-1 and Beam-2 were wrapped with 2 layers of HFRP. Prior to wrapping beam-1 was damaged on tension side in middle length zone and beam-2 on tension face as well as on sides throughout the length as described in section 6.4.2. Maximum load taken by beams wrapped with 2 layers of HFRP were 19.25kip and 16.15kip for beam-1 and beam-2 respectively. Cracking observed at 5kip load. Beam-1 with damage only on tension face has more area under loaddeflection curve than beam-2 with damage on tension face as well as on sides.

Debonding failure was observed at a strain level of 0.0102 and 0.0067 for beam- 1 and beam- 2 respectively. This might be due to the wavy nature of the fabric and insufficient resin to fabric ratio. Due to debonding, FRP contribution was not utilized completely. This can be observed by comparing the HFRP strain at failure of beam with the ultimate strain in HFRP i.e., 0.015 that

obtained from the tensile tests on HFRP coupons. The experimental values for load and moment for beam-1 and beam- 2 were $98 \%$ and $83 \%$ respectively of theoretical values calculated using debonding strains. The failure modes are shown in Fig 6.14 and 6.15. 
Batch-2: Maximum load taken by beams wrapped with 2 layers and 3 layers of HFRP were 19.99kip and 17.36kip respectively. Cracking observed at 7kip load for 2 layered and 3 layered beams. 2 layered beam was pre-cracked, wrapped, loaded until crack and then crack sealed before loading and as a result of the crack seal, increase in stiffness was observed (Appendix F).

Debonding failure was observed at a strain level of 0.0082 for 2 layered beam and shearcompression failure following debonding was observed at a strain level of 0.0069 for 3 layered beam. Also, delamination between HFRP layers was noticed due to which the beam seems to be acted as 2 layered rather than 3 layered. Similar failure pattern was also observed during pull-off tests. This might be due to the wavy nature of the fabric and insufficient resin to fabric ratio. Due to debonding, FRP contribution was not utilized completely. This can be observed by comparing the HFRP strain at failure of beam with the ultimate strain in HFRP i.e., 0.015 that obtained from the tensile tests on HFRP coupons. The experimental values for load and moment for 2-layered and 3-layered were $98 \%$ and $76 \%$ of theoretical values calculated using debonding strains. The failure modes are shown in Fig 6.16 and 6.17. 


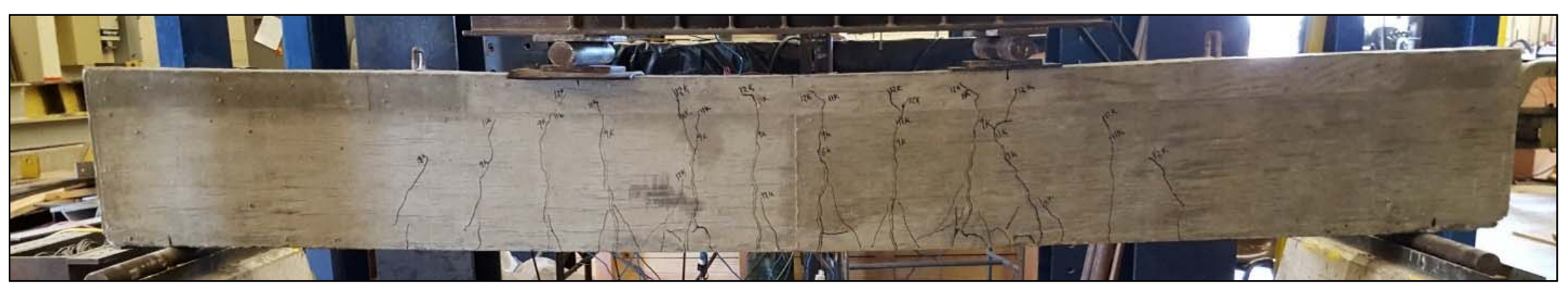

Figure 6-7 Failure of Control Beam from Batch-1

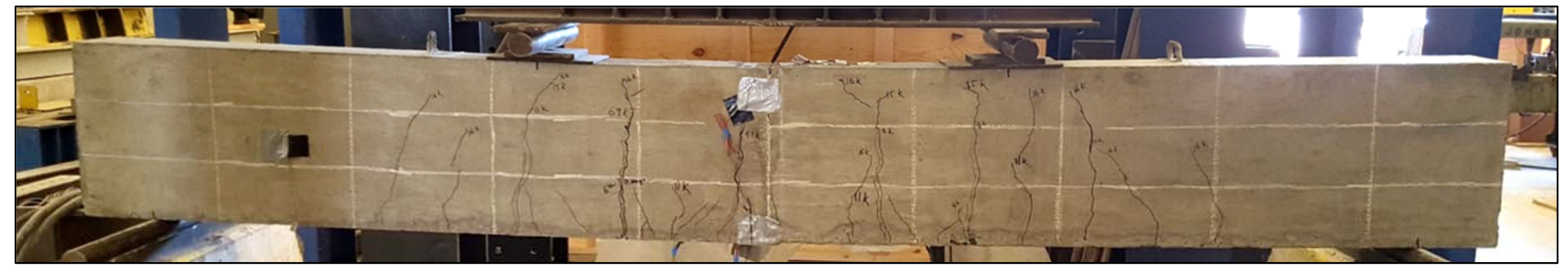

Figure 6-8 Failure of Control Beam-1 from Batch-2

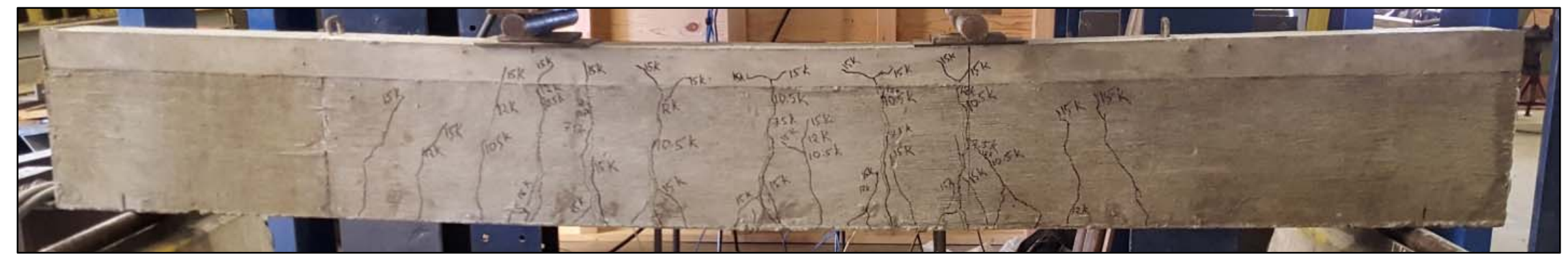

Figure 6-9 Failure of Control Beam-2 from Batch-2 


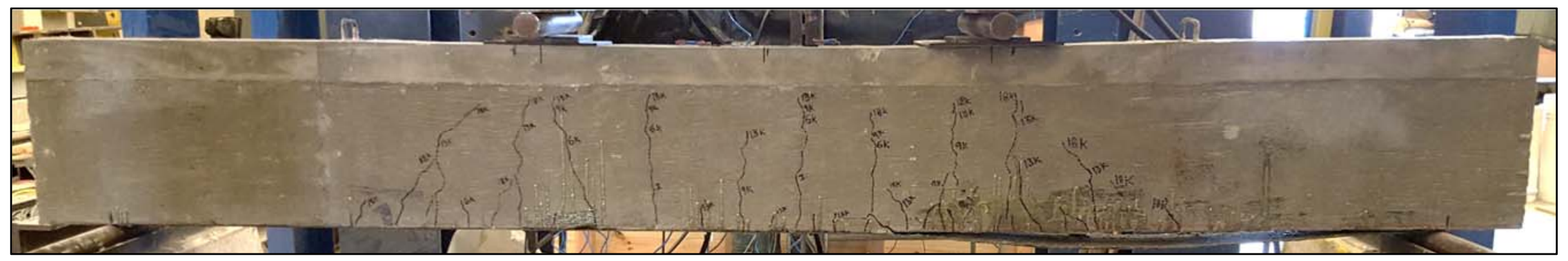

Figure 6-10 Failure of 2 Layered CFRP Wrapped Beam-1 from Batch-1

(Damaged concrete on tension face in middle length zone, repaired, pre-cracked, wrapped and loaded)

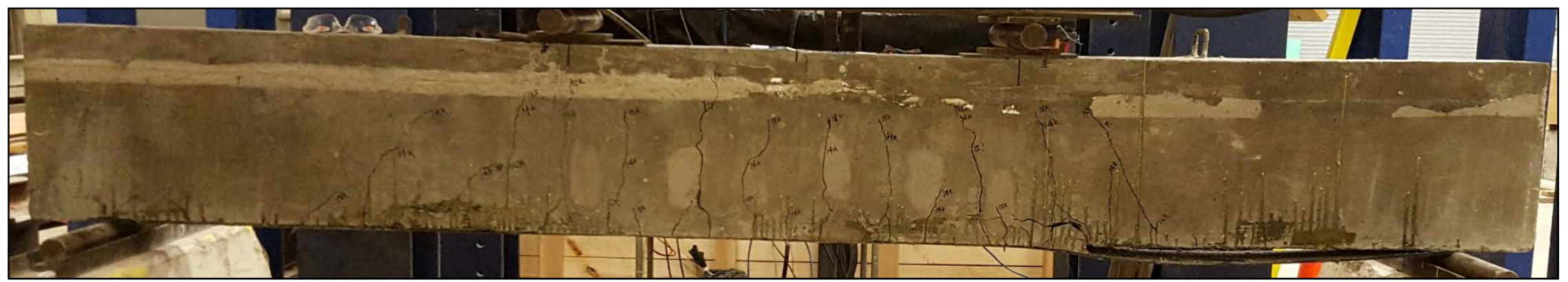

Figure 6-11 Failure of 2 Layered CFRP Wrapped Beam-2 from Batch-1

(Damaged concrete on tension face and also on sides in middle length zone, repaired, pre-cracked, wrapped and loaded)

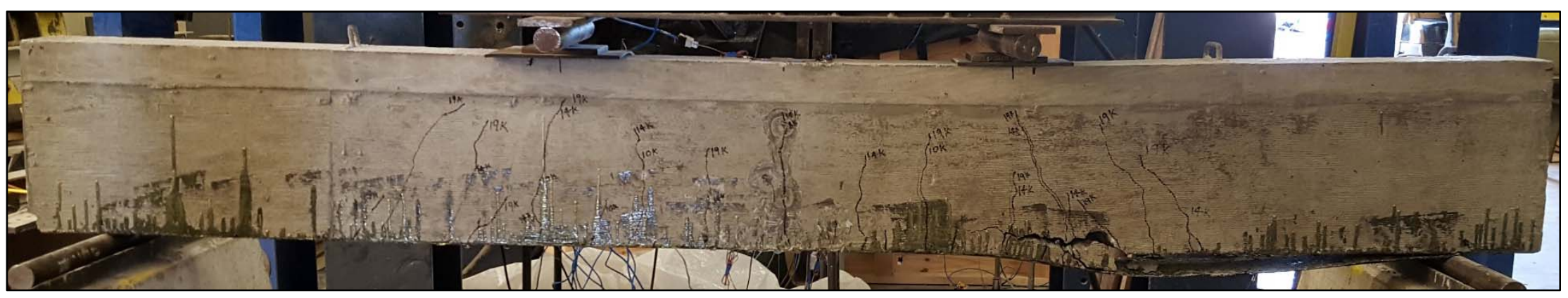

Figure 6-12 Failure of 2 Layered CFRP Wrapped Beam from Batch-2

(Pre-cracked, wrapped, cracked, crack sealed and loaded) 


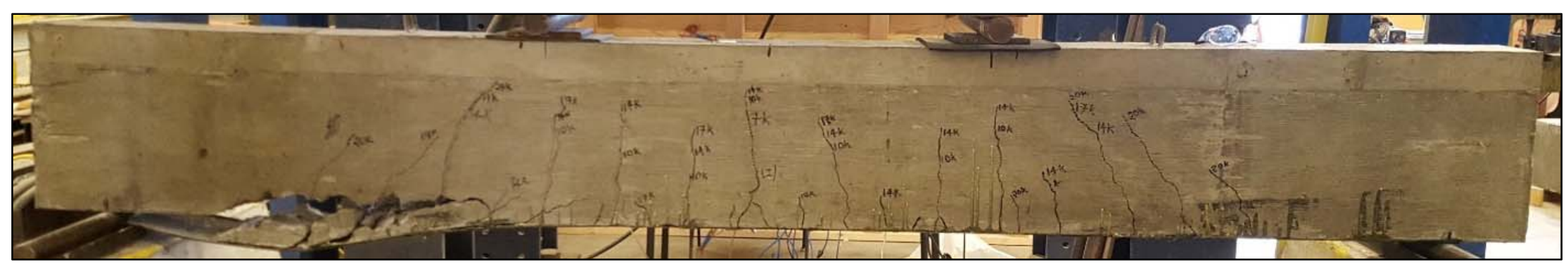

Figure 6-13 Failure of 3 Layered CFRP Wrapped Beam from Batch-2

(Pre-cracked, wrapped, and loaded)

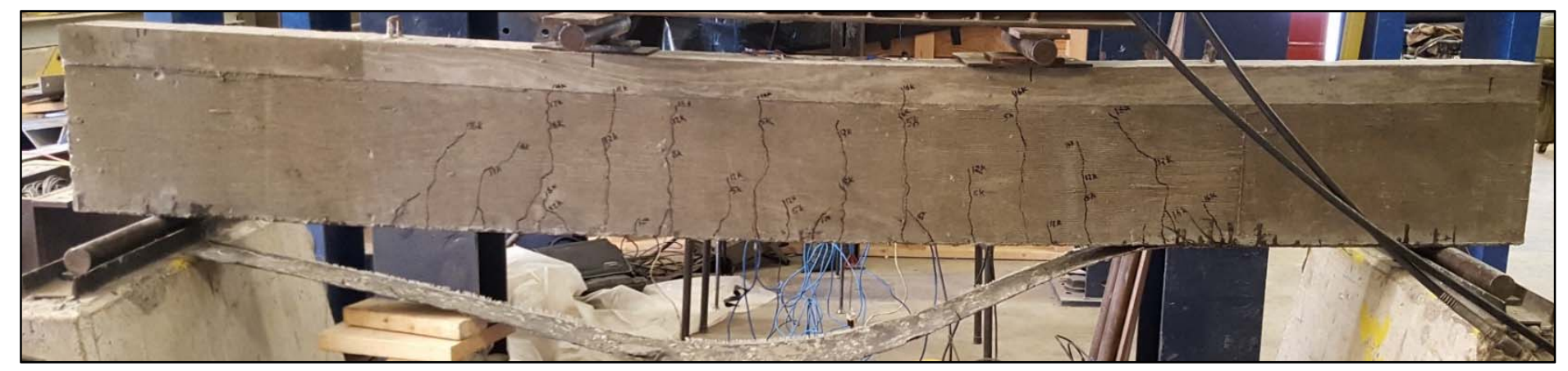

Figure 6-14 Failure of 2 Layered HFRP Wrapped Beam-1 from Batch-1

(Damaged concrete on tension face in middle length zone, repaired, pre-cracked, wrapped and loaded)

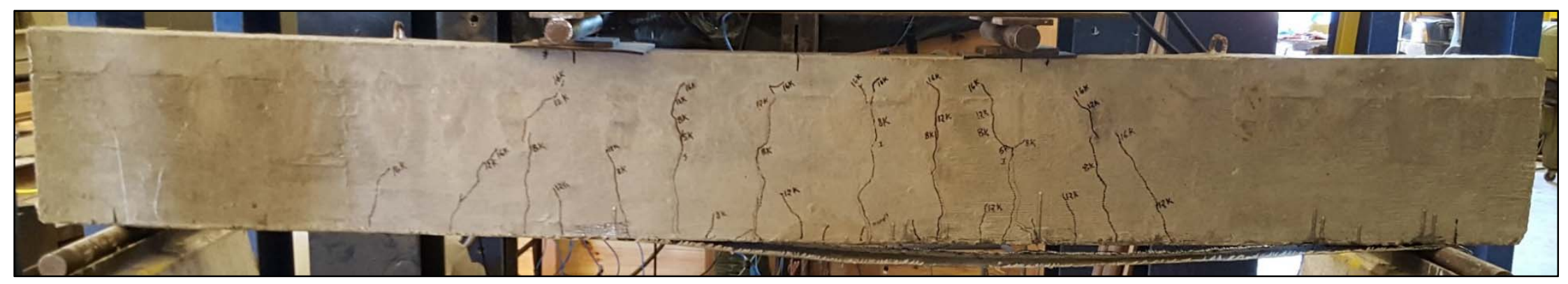

Figure 6-15 Failure of 2 Layered HFRP Wrapped Beam-2 from Batch-1

(Damaged concrete on tension face and also on sides throughout the length, repaired, pre-cracked, wrapped and loaded 


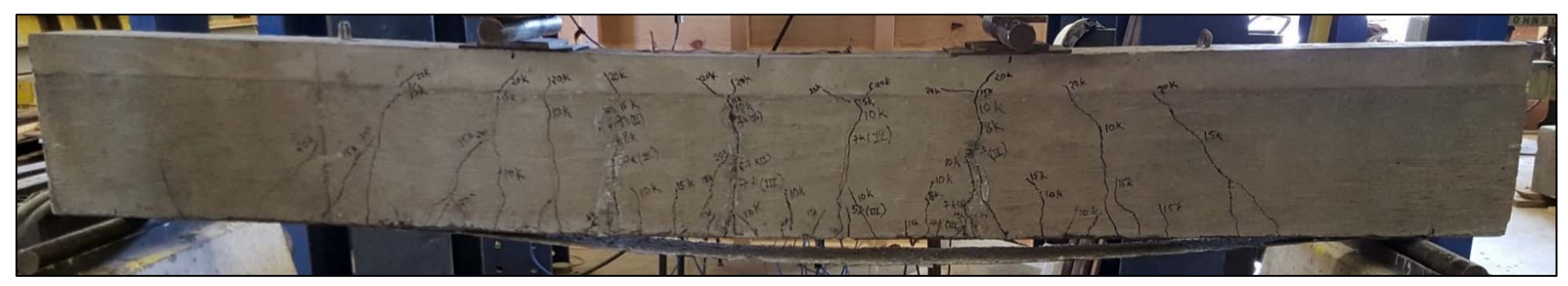

Figure 6-16 Failure of 2 Layered HFRP Wrapped Beam from Batch-2

(Pre-cracked, wrapped, cracked, crack sealed and loaded)

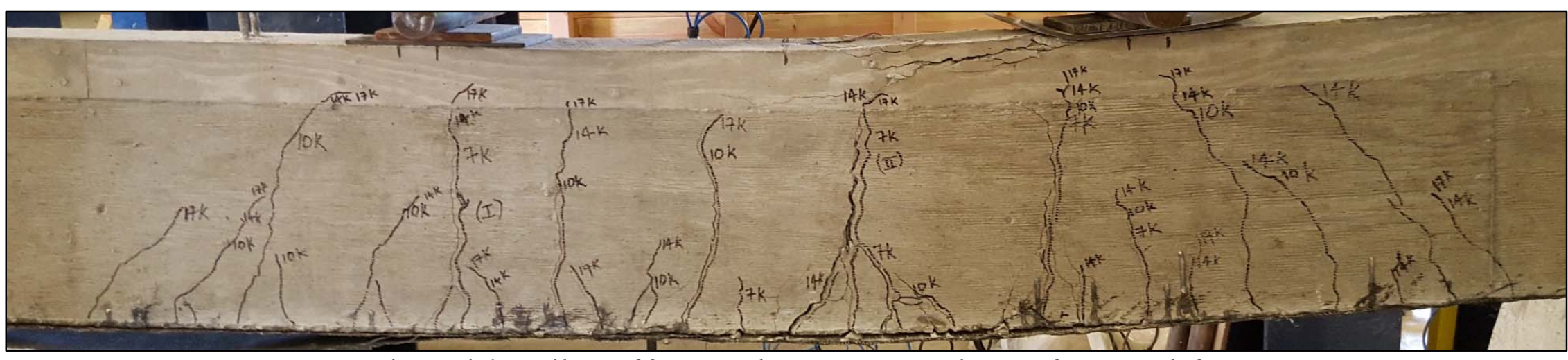

Figure 6-17 Failure of 3 Layered HFRP Wrapped Beam from Batch-2

(Pre-cracked, wrapped, cracked, crack sealed and loaded)

\subsection{Summary}

In this chapter, the test results and analysis of the four point bending tests conducted on the reinforced concrete beams confined with

FRP wraps were discussed. The effect of flexural strengthening was presented in the form of tables and graphs. Variation in strength, stiffness and ductility due to FRP wraps on pre-cracked have been provided and the failure modes of the specimens were shown in Fig. 6.7 to 6.17 . The results obtained were analyzed and discussed. 


\section{CONCLUSIONS}

\subsection{Introduction}

Damaged concrete members (cylinders and beams) were strengthened with CFRP-epoxy and HFRP-polyurethane fabric-resin systems to study the effect of the rehabilitation schemes. The tests conducted in this research work are as given below:

$\checkmark$ Sixty (60) FRP coupon specimens were tested to determine the tensile properties such as strength, stiffness, and strain of the two fabric-resin systems, CFRP-epoxy and HFRPpolyurethane.

$\checkmark$ Forty-three (43) FRP pull-off samples were tested to evaluate the bond strength between concrete substrate and FRP overlay.

$\checkmark$ Thirty-nine (39) plain concrete cylinders were tested with and without FRP wrappings to determine the confinement effect with increase in number of layers and strength recovery with repair of damaged FRP repair.

$\checkmark$ Twenty-two (22) split-bonded concrete cylinders were tested with and without wrappings to evaluate the strength recovery.

$\checkmark$ Eleven (11) reinforced concrete beams either pre-cracked or damaged were tested with and without wrappings.

Results such as strength, stiffness, strain, and failure modes were obtained from all the abovementioned tests for varying parameters and load conditions. Comparisons were made to determine the effect of confinement in cylinders and flexural strength enhancement in beams. Conclusions based on all the test results for different types of tests are provided in this chapter. 


\subsection{Material Properties}

\subsubsection{Tension Tests on FRP Coupon Specimens}

- CFRP-epoxy has an average stress of 132ksi, stiffness of $14 \mathrm{msi}$ and ultimate failure strain of $1.2 \%$. HFRP pre-preg has an average stress of $144 \mathrm{ksi}$, stiffness of $8.87 \mathrm{msi}$ and ultimate failure strain of $1.5 \%$.

- CFRP-epoxy coupons were about 1.5 times stronger per unit width compared to HFRP coupons (3.12kip vs. 1.97kip per 0.75 " width for 1 layer coupons). And were about 1.5 times stiffer compared to HFRP coupons (8.87msi vs. 14msi).

- Near failure loads, some layer separations were noted only in multiple HFRP layer coupon specimens.

\subsubsection{Pull-off Tests on FRP Overlay}

- Pull-off tests indicated a good bond strength between concrete substrate and FRP overlay with a value of up to 272psi and 384psi for HFRP and CFRP, respectively.

- Very few repair material failure (layer separations) were noted only in multiple HFRP layer specimens. Concrete substrate failure very close to the substrate/overlay interface for both CFRP and HFRP specimens.

- With respect to above values, bond strengths increased by $72 \%$ and $20 \%$ for power wash prepared surfaces due to increased surface area and better wetting for HFRP and CFRP, respectively.

- Bi-axial HFRP fabric has woven fibers and the surface undulations of fibers bond well with power washed and pitted concrete surface that requires additional amount of primer for better surface wetting and bonding. 


\subsection{Compression Tests on Concrete Cylinders}

\subsubsection{Non-Wrapped Cylinders}

\subsubsection{Plain Cylinders}

- Compression tests on non-wrapped plain cylinders showed a strength of 154kip (5.46ksi) for batch-1 and 181kip (6.40ksi) for batch-2.

\subsubsection{Split-Bonded Cylinders:}

- Split-bonded cylinders of batch-1 carried 111kip (3.94ksi) as compared to plain cylinders carrying $154 \mathrm{kip}$ (5.46ksi) showing a reduction of $28 \%$ due to split-bonding.

- Split-bonded cylinders of batch-2 carried 126kip (4.47ksi) as compared to plain cylinders carrying $181 \mathrm{kip}(6.4 \mathrm{ksi})$ showing a reduction of $30 \%$ due to split-bonding.

As compared to plain cylinders, split bonded cylinders exhibited a $\sim 30 \%$ reduction in compressive strength.

\subsubsection{FRP Wrapped Cylinders}

\subsubsection{Plain Cylinders}

* For 1,2 and 3 layers of CFRP wraps

- The per layer strength increase was $(67,89$, and $73 \%)$ for $\boldsymbol{f}_{c}^{\prime}=5.46 \mathrm{ksi}$ and $(37,71$, and $58 \%$ ) for $\boldsymbol{f}_{c}^{\prime}=6.4 \mathrm{ksi}$.

- For both concrete compressive strengths, the per layer percentage strength increase was found to be highest for 2 layers of wrap and lowest for 1 layer of wrap.

\section{For 1,2 and 3 layers of HFRP wraps}

- The per layer strength increase was $(34,37$, and $46 \%)$ for $\boldsymbol{f}_{c}^{\prime}=5.46 \mathrm{ksi}$. 
- The per layer strength increase was found to be highest for 3 layers of wrap and lowest for 1 layer of wrap.

Both HFRP with polyurethane resin system and CFRP with epoxy resin system provided necessary strength increase in cylinders as indicated by experimental to theoretical ratios being closer to one or above.

* Cylinders wrapped with CFRP fabrics showed CFRP rupturing along the hoop direction. However, cylinders with HFRP fabrics showed localized axial fabric buckling along the glass (axial) direction and carbon fabric rupturing in the hoop direction.

\subsubsection{Split-Bonded Cylinders:}

Based on the above results, only two layer wraps were considered (as a minimum) for further study with split-bonded cylinders based on combination of strength and cost-effectiveness criteria for real field applications.

\section{For 2 layers of CFRP Wraps (Batch-2, $\left.f_{c}^{\prime}=6.4 \mathrm{ksi}\right):$}

- As compared to plain concrete cylinders without wraps, the per layer strength increase for split-bonded cylinders with wraps was $64 \%$. Corresponding per layer strength increase in regular cylinders with similar wrapping was $71 \%$.

- When compared to plain cylinders with 2 layers of wraps, a $6 \%$ strength reduction was noted. (437 kip vs. 412 kip).

\section{For 2 layers of HFRP Wraps (Batch-1, $\left.f_{c}^{\prime}=5.46 \mathrm{ksi}\right):$}

- As compared to plain concrete cylinders without wraps, the per layer strength increase for split-bonded cylinders with wraps was 34\%. Corresponding per layer strength increase in regular cylinders with similar wrapping was 37\%. 
- When compared to plain cylinders with 2 layers of wraps, a $4 \%$ strength reduction was noted. (268 kip vs. 257 kip).

The above results indicate the effectiveness of CFRP and HFRP wraps in achieving confinement related strength increases in damaged, repaired and wrapped cylinders (split bonded and wrapped) as compared to similar wrapping in cylinders without any damage.

Note: Strength reduction of $6 \%$ and $4 \%$ were noted in CFRP and HFRP wrapped split-bonded cylinders of $\boldsymbol{f}_{c}^{\prime}=6.4 \mathrm{ksi}$ and $\boldsymbol{f}_{c}^{\prime}=5.46 \mathrm{ksi}$ respectively

\subsection{Flexural Strength Enhancement of RC Beams}

Beams (6" x 15" x 120") have been strengthened for flexure with both CFRP and HFRP fabrics. These beams were pre-loaded to slightly above their first cracking load following which they were bonded at the beam bottom with different number of FRP layers as designed. Following FRP strengthening, beams were tested in several cycles to failure.

Note: Actual FRP strains observed during failure are (62\% to $93 \%$ for HFRP and $45 \%$ to $60 \%$ for CFRP) lower than the de-bonding strains calculated as per ACI 440. Hence, in theoretical calculations, $60 \%$ of effective debonding strains were used.

\subsubsection{CFRP-Epoxy (pre-cracked and wrapped)}

- The experimental moment values were within $10 \%$ of the theoretical values for both concrete batches, with ratios of up to 1.04 for 2 layered and 0.91 for 3 layered beams.

- The failure was in the form of end cover separation with FRP de-bonding. It was observed that the failure was influenced by lower bond strength due to high resin viscosity. 
- CFRP strains (recorded) at failure were in a range of $45 \%$ to $60 \%$ of the de-bonding failure strains as computed by equations from $\mathrm{ACI}\left(\boldsymbol{\varepsilon}_{\boldsymbol{f} d}, \mathrm{ACI} 440\right)$.

\subsubsection{HFRP-Polyurethane (pre-cracked and wrapped)}

- The experimental moment values were within $25 \%$ of the theoretical values for both concrete batches, with ratios of up to 0.98 for 2 layered and 0.76 for 3 layered beams.

- The failure was in the form of FRP de-bonding and delamination was also seen between multiple layers.

- HFRP strains (recorded) at failure were in a range of $62 \%$ to $93 \%$ of the de-bonding failure strains as computed by equations from $\mathrm{ACI}\left(\boldsymbol{\varepsilon}_{\boldsymbol{f} d}, \mathrm{ACI} 440\right)$.

\subsubsection{Effect of Beam Crack Repair through Resin Injection}

* Crack formations in beams bonded with FRP layers at the bottom tension zone and loaded previously were injected with resin and cured. Subsequent loading of the resin-injected beams showed an increase in beam stiffness (Appendix F).

* Both CFRP and HFRP fabrics helped improve the flexural strength of the RC beams. However, mostly de-bonding failures were observed.

Thick layers of primer would help to improve the bond strength and hence flexural strength enhancements. 


\section{REFERENCES}

[1] V. M. Karbhari, J. W. Chin, D. Hunston, B. Benmokrane, T. Juska, R. Morgan, J. J. Lesko, U. Sorathia and a. D. Reynaud, "Durability Gap Analysis for Fiber-Reinforced Polymer Composites in Civil Infrastructure," JOURNAL OF COMPOSITES FOR CONSTRUCTION, ASCE, pp. 238-247, 2003.

[2] R. Cusson and Y. Xi, "THE BEHAVIOR OF FIBER-REINFORCED THE BEHAVIOR OF FIBER-REINFORCED TEMPERATURE ENVIRONMENTAL CLIMATES," Colorado Department of Transportation (CDOT), 2002.

[3] P. Woraphot, "Durability of Concrete Beams with FRP Wraps," West Virginia University, Morgantown, 2003.

[4] G. Wu, Z. S. Wu, Z. T. Lu and Y. B. Ando, "Structural Performance of Concrete Confined with Hybrid FRP Composites," Journal of REINFORCED PLASTICS AND COMPOSITES, Vol. 27, p. 1323-26, 2008.

[5] R. R. Rajappa, "BEHAVIOR OF FRP WRAPPED CONCRETE CYLINDERS," West Virginia University, Morgantown, 2004.

[6] A. Nanni, M. Norris and N. Bradford, "Lateral Confinement of Concrete using FRP Reinforcement," in Fiber Reinforced- Plastic Reinforcement for Concrete Structures, International Symposium, 1993. 
[7] F. Picher, P. Rochette and P. Labossiere, "Confinement of concrete cylinders with CFRP," Fiber Composites in Infrastructure, pp. 829-841, 1996.

[8] G. Campione and N. Miraglia, "Strength and strain capacities of concrete compression members reinforced with FRP," Cement and Concrete Composites 25(1), p. 31-41, 2003.

[9] R. Eid, N. Roy and P. Paultre, "Normal- and high-strength concrete circular elements wrapped with FRP composites," Journal of Composites for Construction 13(2), p. 113-124, 2009.

[10] S. Zhang, L. Ye and M. Y. Wing, "A study on polymer composite strengthening system for concrete columns," Applied Composite Materials, p. 7:125-138, 2000.

[11] R. Sen and G. Mullins, "Application of FRP composites for underwater piles repair," Composites Part B:engineering, pp. 751-758, 2007.

[12] E. Thorhallsson, S. Kubens and A. Konradsson, "Concrete Cylinders Confined with Basalt Fibre Reinforced Polymer," Reykjavik University, 2011.

[13] S. Homan and S. Sheikh, "Durability of fiber Reinforced Polymers used in Concrete Structures," in Proceedings of the Third International Conference on Advanced Composite Materials in Bridges and Structures, Ottawa (Ontario) Canada, 2000.

[14] S. Kshirsagar, "Durability of Fiber Reinforced Composite Wrap System for the Rehabilitation of Concrete Structures," West Virginia University, Morgantown, 1998. 
[15] Y. Chen, J. F. Davalos, I. Ray and H. Y. Kim, "Durability of GFRP reinforcing bars embedded in moist concrete," Journal of Composites for Construction ASCE, pp. Vol. 10, No. 4, pp. 279-286, 2006.

[16] P. A. Wagner, B. J. Little, K. R. Hart and R. I. Ray, "Biodegradation of Composite Materials," International Biodeterioration \& Biodegradation, pp. 125-132, 1996.

[17] H. Toutanji and P. Balaguru, "Durability characteristics of concrete columns wrapped with fiber tow sheets," J. Mater. Civ. Eng., pp. 10(1), 52-57, 1998.

[18] J. Zhou, X. Chen and C. Shixue, "Effect of Different Environments on Bond Strength of Glass Fiber-Reinforced Polymer and Steel Reinforcing Bars," KSCE Journal of Civil Engineering, pp. 16(6):994-1002, 2012.

[19] H. Wei, Z. Wu, X. Guo and F. Yi, "Experimental study on partially deteriorated concrete columns confined with CFRP," Engineering Structures, p. 31(10):2495-2505, 2009.

[20] M. R. Ehsani, H. Saadatmanesh and S. Tao, "Design recommendation for bond of GFRP rebars to concrete," Journal of Structural Engineering, ASCE, pp. Vol. 122, No. 3, pp. 247254, 1996.

[21] J. Teng and L. Lam, "Behavior and modeling of fiber reinforced polimer-confined concrete," Journal of Structural Engineering, p. 130(11): 1713-1723, 2004.

[22] O. Chaallal, M. Hassan and M. LeBlanc, "Circular Columns Confined with FRP: Experimental versus Predictions of Models and Guidelines," JOURNAL OF COMPOSITES FOR CONSTRUCTION, pp. 10:4-12, 2006. 
[23] T. El Maaddawy, "Strengthening of eccentrically loaded reinforced concrete columns with fiber-reinforced polymer wrapping system: Experimental investigation and analytical modeling," J. Compos. Constr., pp. 13-24, 2009.

[24] X. Song, X. Gu, Y. Li, T. Chen and W. Zhang, "Mechanical behavior of FRP-strengthened concrete columns subjected to concentric and eccentric compression loading," J. Compos. Constr., pp. 336-346, 2013.

[25] V. Alecci, S. B. Bati and G. Ranocchiai, "Concrete columns confined with CFRP wraps," Materials and Structures, p. 47:397-410, 2014.

[26] J. F. Davalos, A. Chen, I. Ray and J. R. Levan, "Comprehensive Study on Using Externally Bonded FRP Composites for the Rehabilitation of Reinforced Concrete T-Beam Bridges," JOURNAL OF INFRASTRUCTURE SYSTEMS, ASCE, vol. 18, no. 2, pp. 89-102, 2012.

[27] A. Mirmiran, M. Shahawy, A. Nanni and V. Karbhari, "Bonded repair and retrofit of concrete structures using FRP composites recommended construction specifications and process control manual," NCHRP Rep. 514, Transportation Research Board, Washington, DC, 2004.

[28] J. F. Bonacci and M. Maalej, "Externally bonded FRP for servicelife extension of RC infrastructure," J. Infrastruct. Syst., pp. 6(1) 41-51, 2000.

[29] H. Saadatmanesh and M. Ehsani, "RC beams strengthened with GFRP plates. I: Experimental study," J. Struct. Engrg., ASCE, pp. 117(11), 3417-3433, 1991. 
[30] M. A. Shahawy and T. Beitelman, "'Structural repair and strengthening of damaged prestressed concrete bridges utilizing externally-bonded carbon materials," in Proc., 41st Int. SAMPE Symp. and Exhibition, 1996.

[31] S. Giuseppe, B. Francesco, S. Fabio and R. N. Swamy, "Structural effectiveness of FRP materials in strengthening RC beams," Engineering Structures, vol. 99, pp. 631-641, 2015.

[32] H. Toutanji, P. Saxena, L. Zhao and O. Teng, "Prediction of interfacial bond failure of FRPconcrete surface," J Compos Constr, p. 11 (4):427-436, 2007.

[33] S. Masoud, K. Soudki and T. Topper, "Postrepair Fatigue Performance of FRP-Repaired Corroded RC Beams: Experimental and Analytical Investigation," Journal of Composites for Construction, ASCE, pp. 9:441-449, 2005.

[34] Y.-F. Wu and Y. Huang, "Hybrid Bonding of FRP to Reinforced Concrete Structures," JOURNAL OF COMPOSITES FOR CONSTRUCTION, ASCE, pp. 12(3):266-273, 2008.

[35] J. G. Teng, J. F. Chen, S. T. Smith and L. Lam, FRP strengthened RC structures, Chichester, U.K.: Wiley, 2002.

[36] A. Tounsi, H. T. Daouadji, S. Benyoucef and A. E. bedia, "Interfacial stresses in FRP-plated RC beams: Effect of adherend shear deformations," International Journal of Adhesion \& Adhesives, p. 343-351, 2009.

[37] O. Büyüköztürk and T.-Y. Yu, "Understanding and Assessment of Debonding Failures in FRP-Concrete Systems," in Seventh International Congress on Advances in Civil Engineering, Istanbul, Turkey, 2006. 
[38] S. Zhang and J. Teng, "End cover separation in RC beams strengthened in flexure with bonded FRP reinforcement: simplified finite element approach," Materials and Structures , 2015.

[39] D.-U. Choi, T. H.-K. Kang, S.-S. Ha, K.-H. Kim and W. Kim, "Flexural and Bond Behavior of Concrete Beams Strengthened with Hybrid Carbon-Glass Fiber-Reinforced Polymer Sheets," ACI STRUCTURAL JOURNAL, pp. V. 108, No. 1, 90-98, January-February $2011 .$.

[40] T. H.-K. Kang, W. Kim, S.-S. Ha and D.-U. Choi, "Hybrid Effects of Carbon-Glass FRP Sheets in Combination with or without Concrete Beams," International Journal of Concrete Structures and Materials, pp. Vol.8, No.1, pp.27-41, March 2014.

[41] A. F. ABBASI and C. P. SIESS, "EFFECT OF STRESS-STRAIN CHARACTERISTICS OF HIGH-STRENGTH REINFORCEMENT ON THE BEHAVIOR OF REINFORCED CONCRETE BEAM-COLUMNS," UNIVERSITY OF ILLINOIS, URBANA, ILLINOIS, 1969.

[42] K. Soudki, E. El-Salakawy and B. Craig, "Behavior of CFRP Strengthened Reinforced Concrete Beams in Corrosive Environment," JOURNAL OF COMPOSITES FOR CONSTRUCTION, pp. 11:291-298, 2007.

[43] S. J. Bongiorno, "Designing with High Performance Concrete Reinforcing," STRUCTURE magazine, National Council of Structural Engineers Associations (NCSEA), pp. 1-9, October 2014. 
[44] J. E. A. I. Wiss, "Determination of Yield Strength for Nonprestressed Steel Reinforcement," 2013.

[45] Y. Kurama, B. Morgen and Q. Shen, "Stress-strain behaviour of Turkish reinforcing bars from the November 12, 1999 Duzce Earthquake," Department of Civil Engineering and Geological Sciences, University of Notre Dame, Notre Dame, 2000.

[46] America's Infrastructure, March 2017.

[47] ACI 440.2R-08: Guide for the Design and Construction of Externally Bonded FRP Systems for Strengthening Concrete Structures, 2008.

[48] ASTM C39/C39M - 14: Standard Test Method for Compressive Strength of Cylindrical Concrete Specimens, 2014.

[49] ASTM C109/C109M - 16a: Standard Test Method for Compressive Strength of Hydraulic Cement Mortars (Using 2-in. or [50-mm] Cube Specimens), 2016.

[50] ASTM C617/C617M - 15: Standard Practice for Capping Cylindrical Concrete Specimens, 2015.

[51] ASTM D 3039/D 3039M - 00: Standard Test Method for Tensile Properties of Polymer Matrix Composite Materials, 2000.

[52] ASTM C1583/C1583M - 13: Standard Test Method for Tensile Strength of Concrete Surfaces and the Bond Strength or Tensile Strength of Concrete Repair and Overlay Materials by Direct Tension (Pull-off Method), 2013. 


\section{APPEndiX A: Stress-Strain CuRVes of Steel Rebar}

Based on concrete strength, reinforcement ratio, strain at peak load, load at peak strain and failure modes, the stress value in steel bar could range between $f_{y}$ and $1.8 f_{y}$. An average value of $1.4 f_{y}$ has been used for theoretical calculations at strain ranges from 0.02 to 0.025 .

In correlation with the stress-strain curves for 60ksi US rebars by Steven J. Bongiorno (2014), Kurama et.al. (2000), and A.F.Abbasi and C.P.Siess (1969), ratio of peak stress to yield stress was observed in a range of 1.4 to 1.8. From Fig A.3 to A.5, varying ranges of stress-strain curves can be seen.

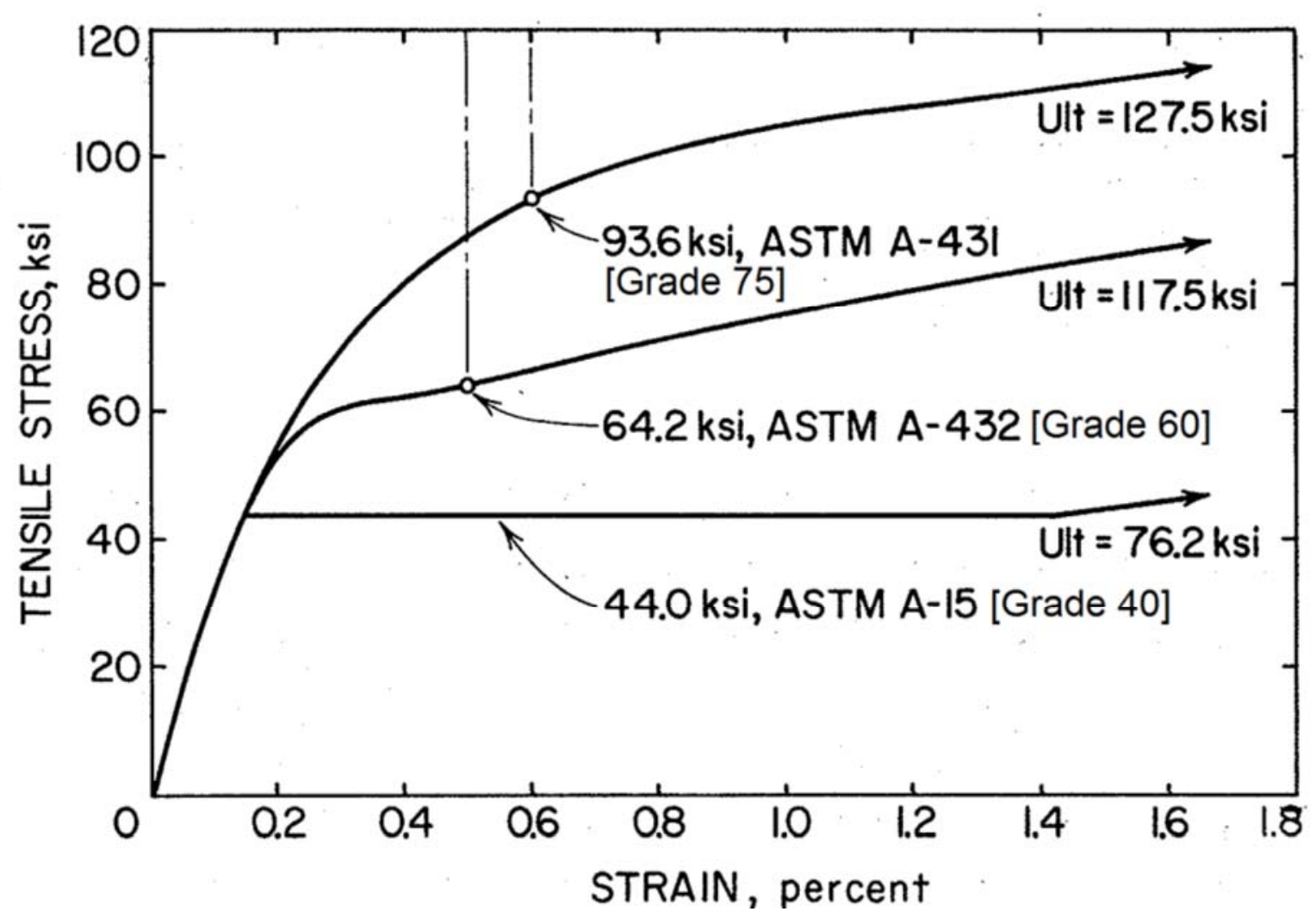

Figure A-1 Actual stress-strain curves for non-prestressed steel bar reinforcement (Grades 75,60 , and 40, top to bottom) that were manufactured during the mid-1960s (Ref: Wiss, Janney, Elstner Associates, Inc. (2013)) 


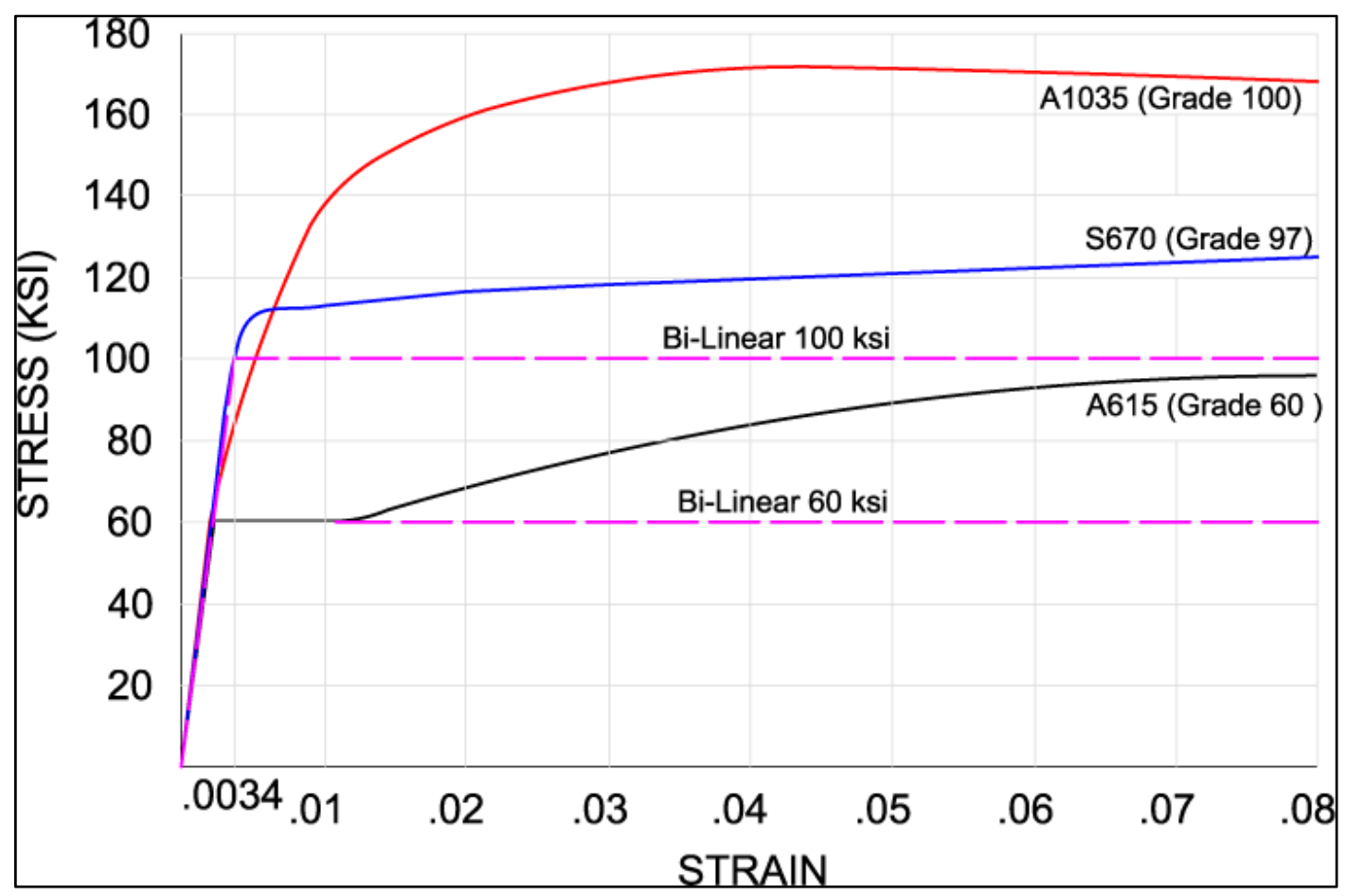

Figure A-2 Comparison of two commercially available high strength reinforcing materials and conventional ASTM A615 (Ref: Steven J. Bongiorno (2014))

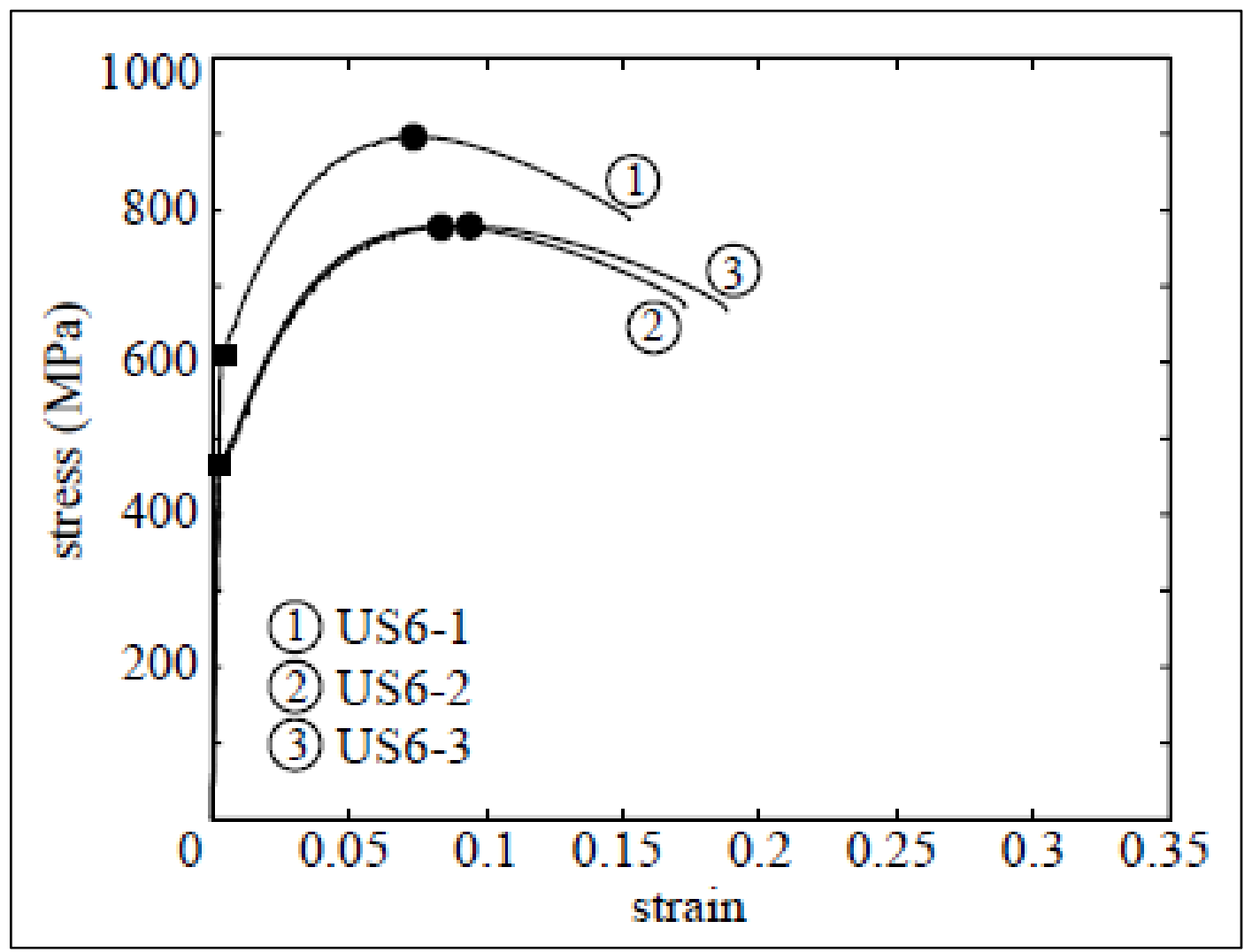

Figure A-3 Stress-Strain Relationships for Grade 60 US bars (Ref: Kurama et.al. (2000)) 


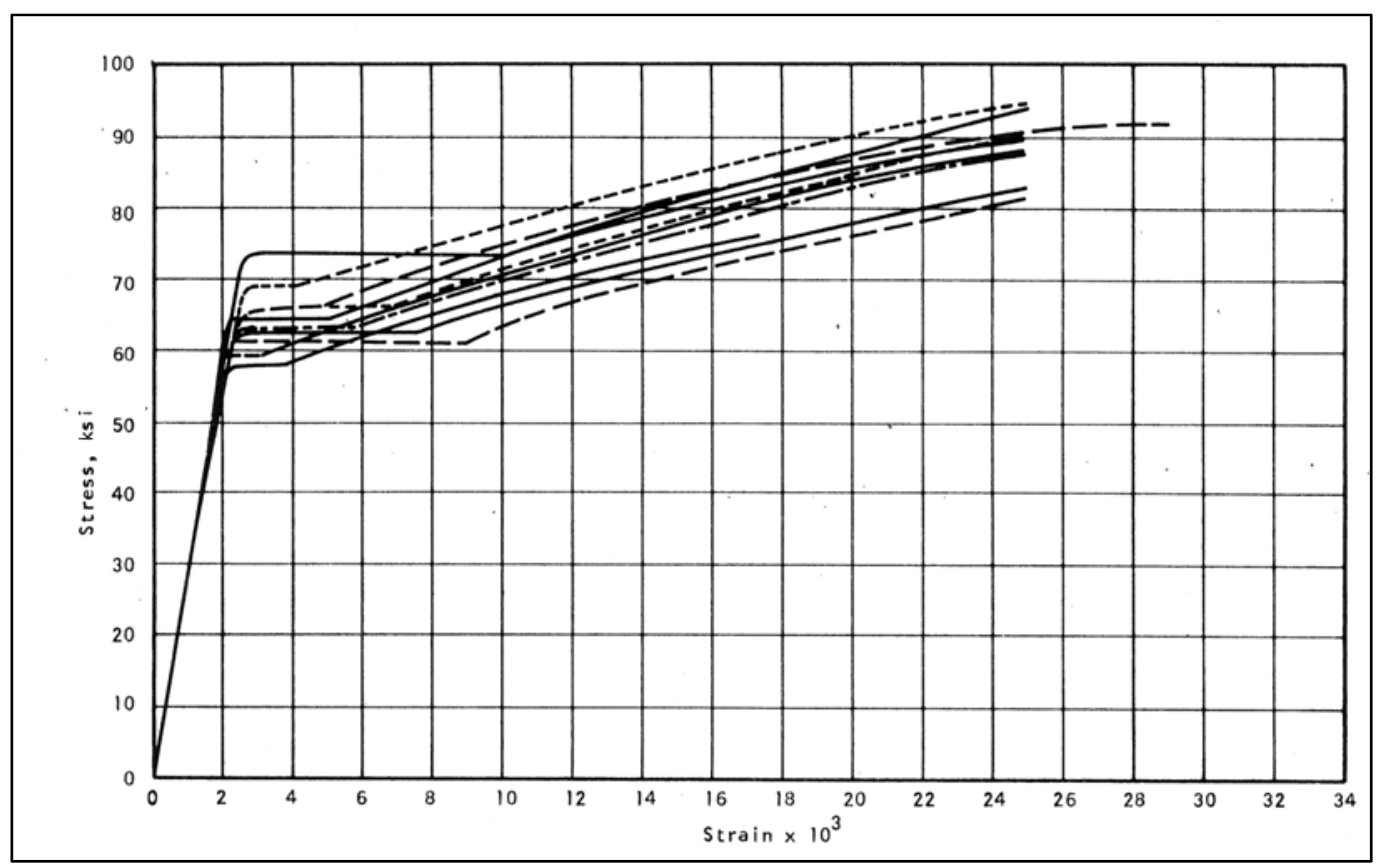

Figure A-4 Stress-Strain Curves for ASTM Grade 60 Steel bars (Ref: A.F.Abbasi and C.P.Siess (1969))

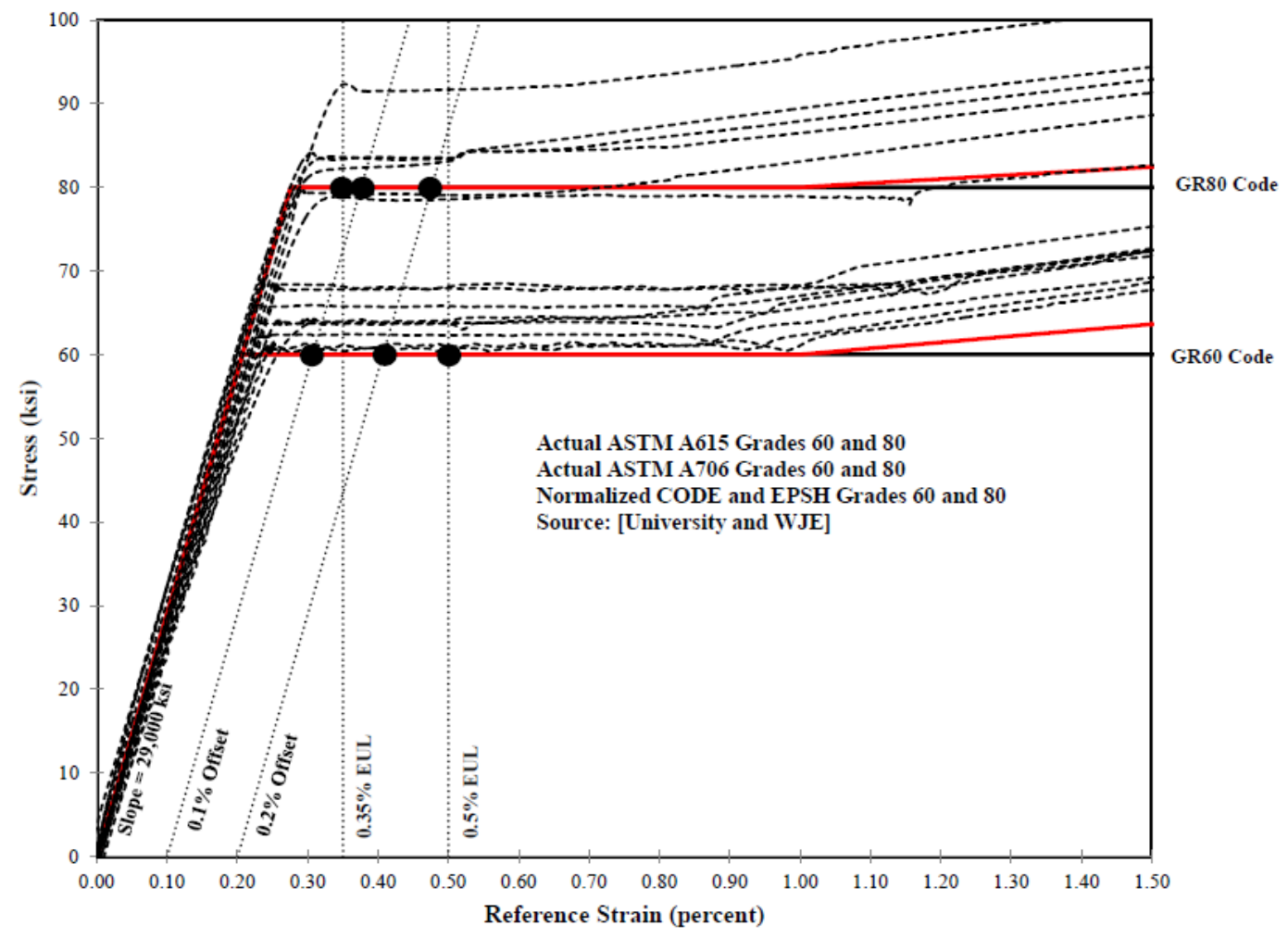

Figure A-5 Stress-Strain Curves for ASTM Grade 60 and 80 Steel bars (Ref: Wiss, Janney, Elstner Associates, Inc. (2013)) 


\section{APPEndiX B: Cylinder Theoretical CAlCulation}

Confinement model according to ACI 440.2R-08 was used for theoretical calculations of compressive strength of confined concrete cylinders. Sample calculations for concrete cylinders confined with 2 layers of CFRP are presented in this section.

\section{Example:}

> Compression testing on externally strengthened concrete cylinder of Batch I with 2 layers of CFRP wraps:

$$
\begin{aligned}
& \text { According to ACI 440.2R-08: } \frac{f_{c c}^{\prime}}{f_{c o}^{\prime}}=\mathbf{1}+\mathbf{3 . 3} \frac{f_{l}}{f_{c o}^{\prime}} \\
& \boldsymbol{f}_{c \boldsymbol{c}}^{\prime} \quad=\text { compressive strength of confined concrete } \\
& \boldsymbol{f}_{\boldsymbol{c} \boldsymbol{o}}^{\prime} \quad=\text { compressive strength of unconfined concrete } \\
& \boldsymbol{f}_{\boldsymbol{l}} \quad=\text { lateral confined pressure by C/GFRP wraps }=\frac{\mathbf{2 E}_{\boldsymbol{f}} \boldsymbol{n} \boldsymbol{t}_{\boldsymbol{f}} \boldsymbol{\varepsilon}_{\boldsymbol{f}}}{\boldsymbol{D}} \\
& \boldsymbol{E}_{\boldsymbol{f}} \quad=\text { modulus of elasticity of CFRP }=14 \text { msi } \\
& \boldsymbol{n} \quad=\text { number of layers }=2 \\
& \boldsymbol{t}_{\boldsymbol{f}} \quad=\text { thickness of fabric }=0.052 \text { in. (for } 2 \text { layers) } \\
& \boldsymbol{\varepsilon}_{\boldsymbol{f} \boldsymbol{e}} \quad=\text { effective failure strain of fabric }=\boldsymbol{k}_{\boldsymbol{\varepsilon}} \boldsymbol{\varepsilon}_{\boldsymbol{f u}} \\
& \boldsymbol{k}_{\boldsymbol{\varepsilon}} \quad=\text { strain efficiency factor }=0.55 \\
& \boldsymbol{\varepsilon}_{\boldsymbol{f} \boldsymbol{u}} \quad=\text { ultimate failure strain of fabric }=0.015 \\
& \boldsymbol{D} \quad=\text { diameter of cylinder }=6 \text { in. }
\end{aligned}
$$

On substituting values we get, $\boldsymbol{f}_{l}=\mathbf{2 0 0 2} \boldsymbol{p s i}$

Therefore, from the above given confinement model,

$$
f_{c c}^{\prime}=12066.6 p s i
$$




\section{APPENDiX C: STRESS-STRAIn CURVES OF CYLINDERS}

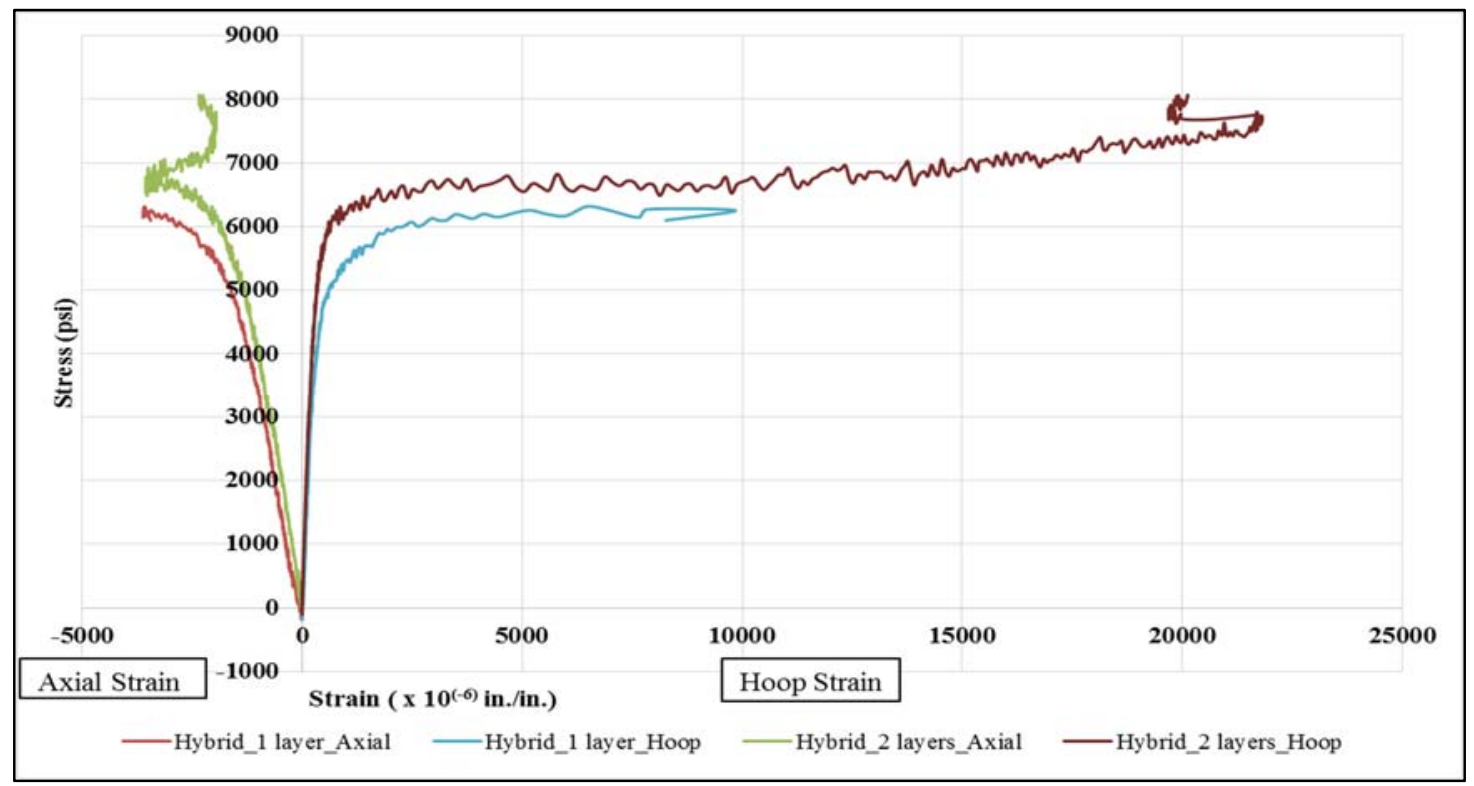

Note: Biaxial fabrics (Hybrid with carbon in hoop direction and glass in axial direction) could exhibit higher axial strains based on resin dominant axial behavior and bond characteristics between concrete and wrap.

Figure C-1 Stress-Strain Curves for Non-Wrapped Full Cylinders, 1, and 2 Layer HFRP Pre-preg Wrapped Full Cylinders of Batch-1

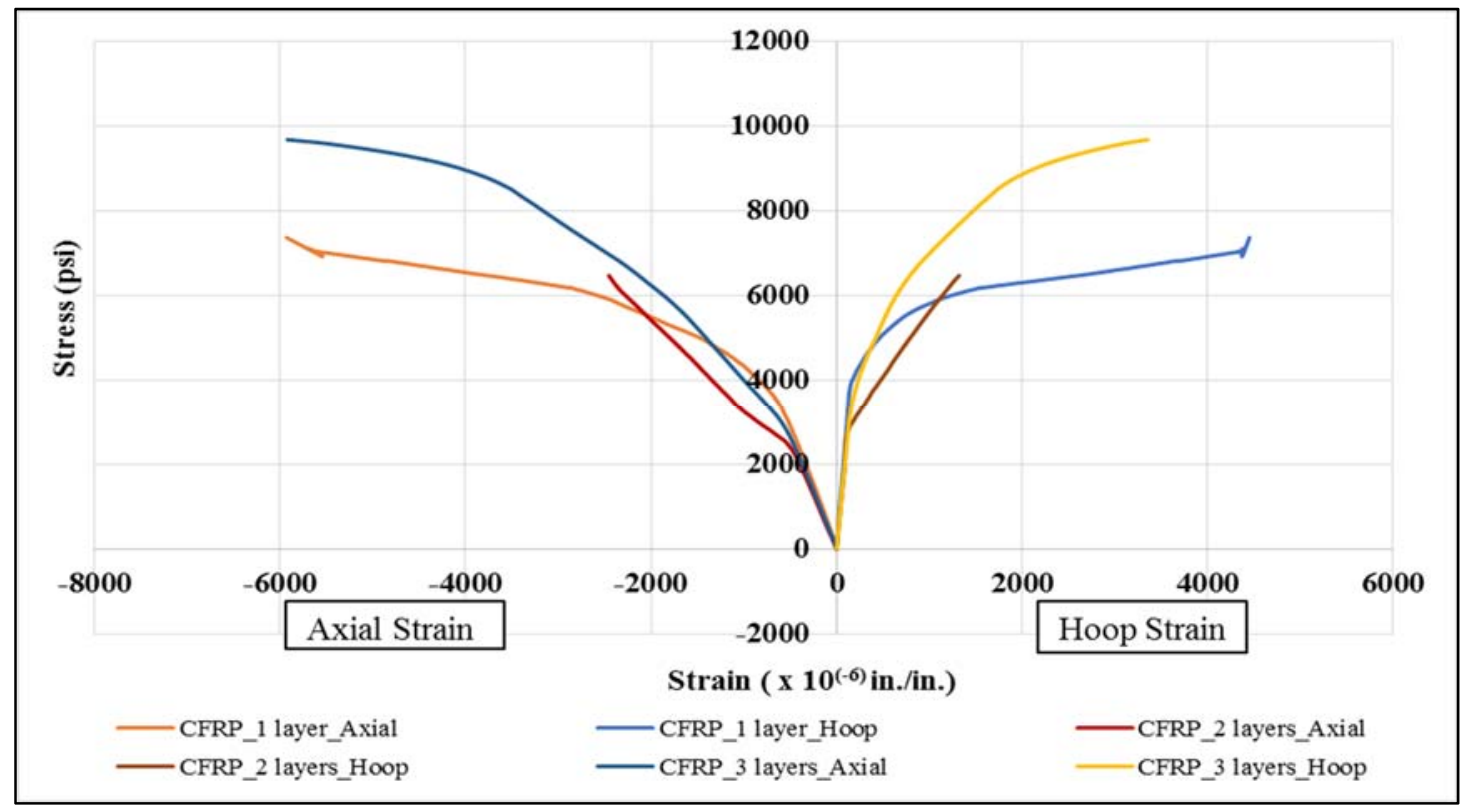

Figure C-2 Stress-Strain Curves for Non-Wrapped Full Cylinders, 1, 2 and 3 Layer CFRP Wrapped Full Cylinders of Batch-1 


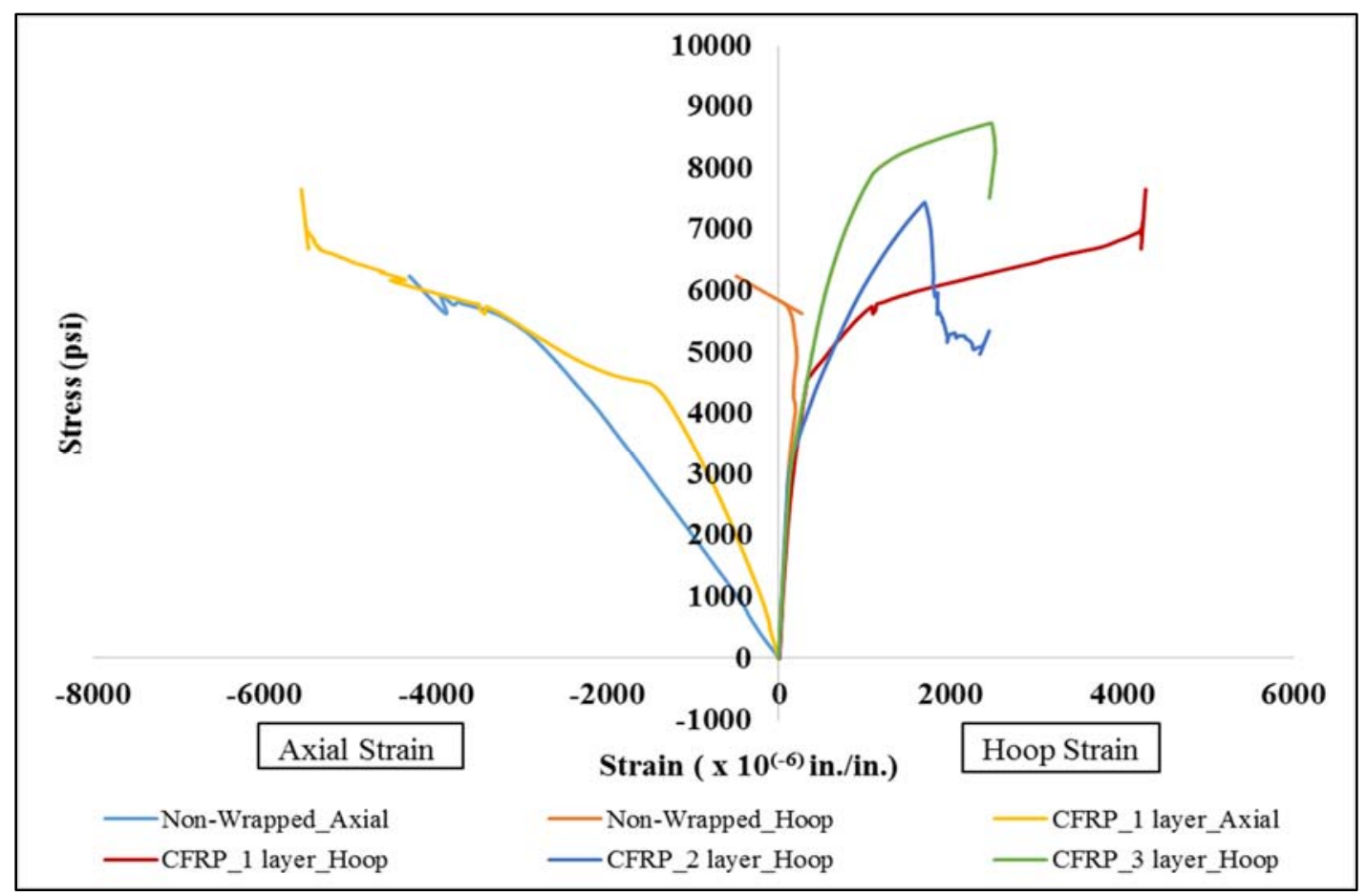

Figure C-3 Stress-Strain Curves for Non-Wrapped Full Cylinders, 1, 2 and 3 Layer CFRP Wrapped Full Cylinders of Batch-2

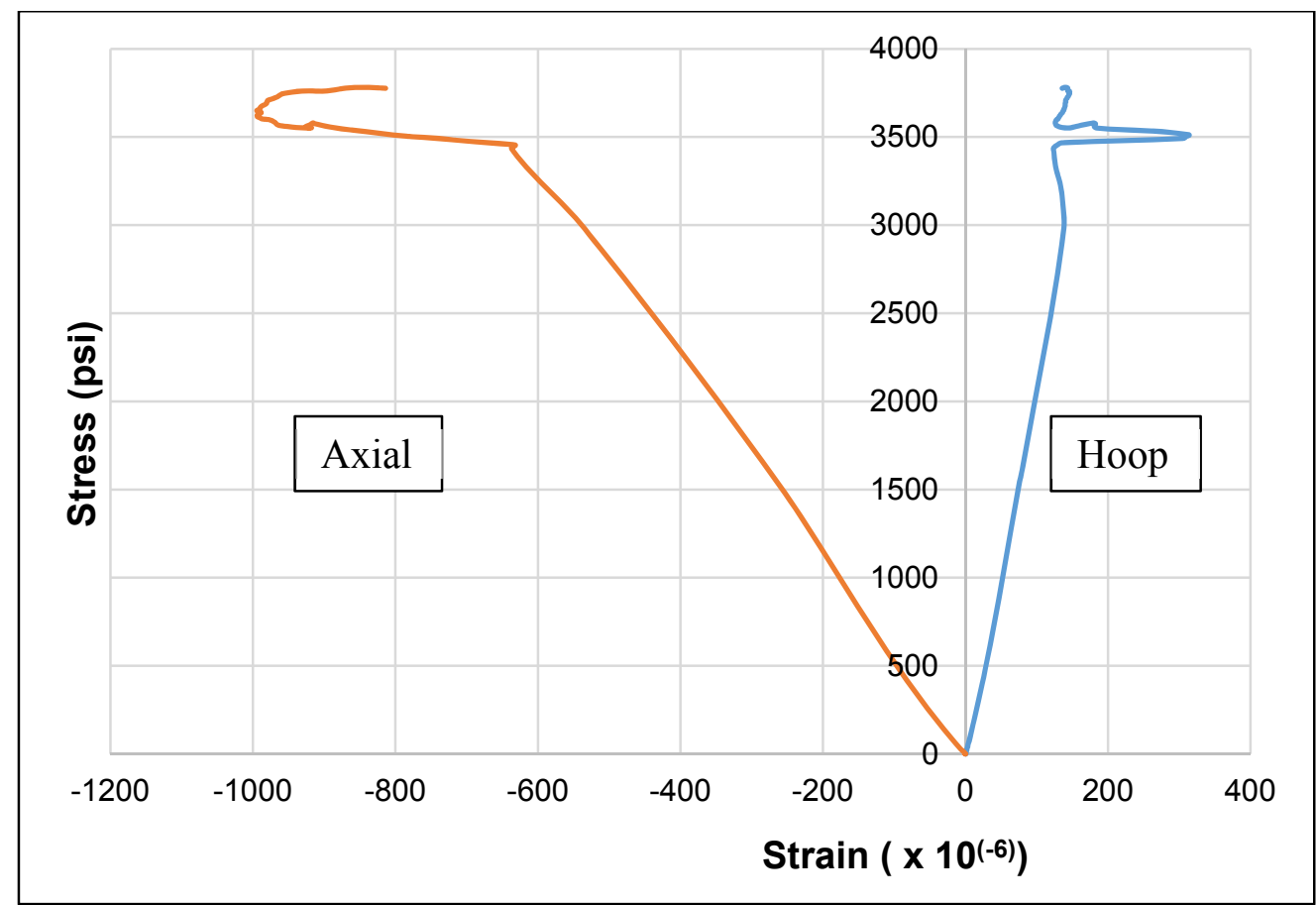

Figure C-4 Stress-Strain Curve for Non-Wrapped Split-Bonded Cylinders of Batch-1 


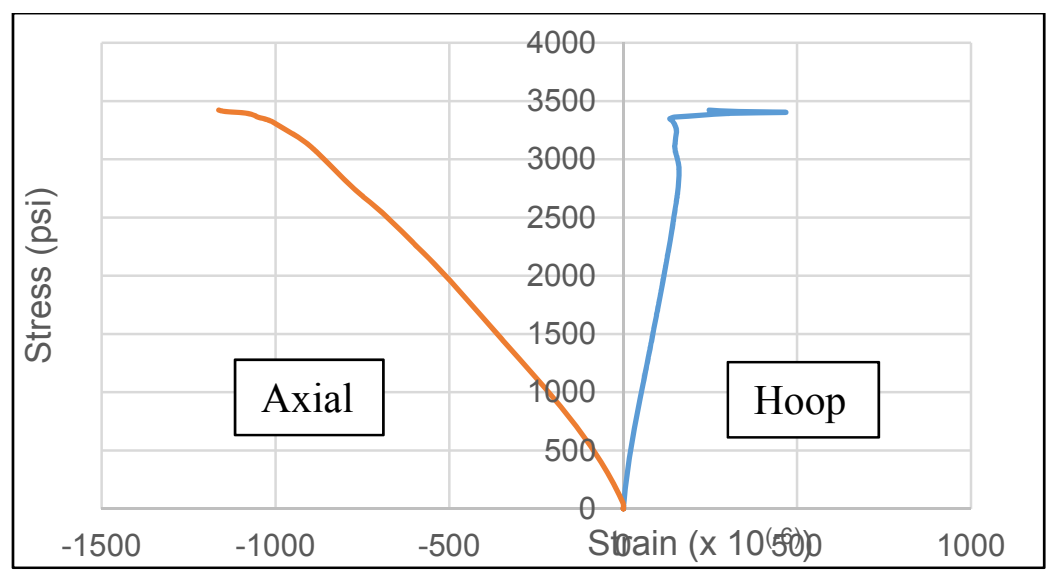

Figure C-5 Stress-Strain Curve for Non-Wrapped Split-Bonded Cylinders of Batch-2

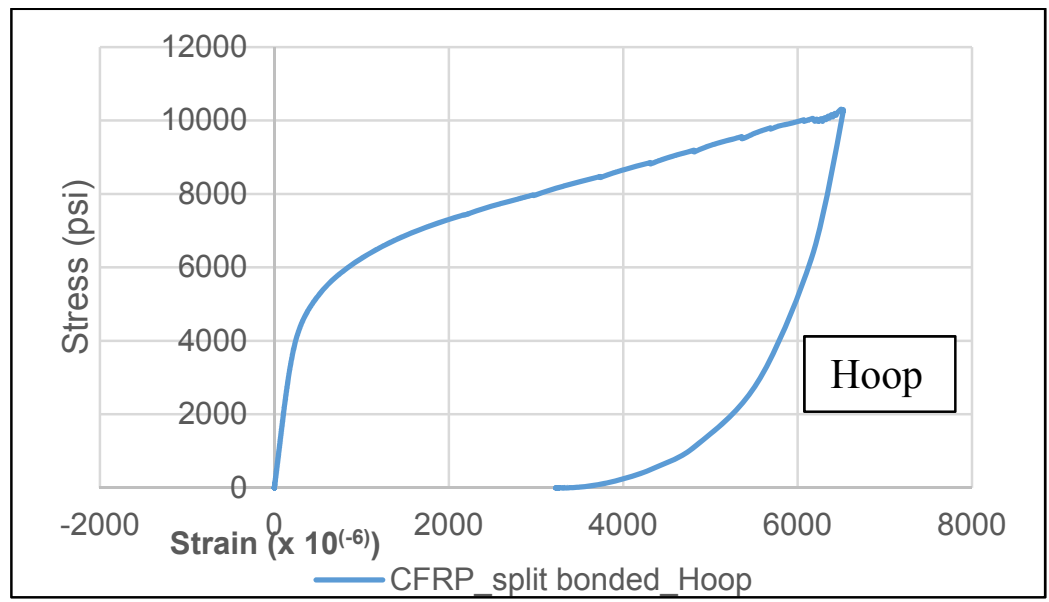

Figure C-6 Stress-Strain Curve for CFRP Wrapped Split-Bonded Cylinders of Batch-2 


\section{APPENDIX D: COMPARISONS OF COMPRESSION TEST RESUlTS OF CONCRETE CYLINDERS}

Table D-1 Compressive Strength of HFRP Pre-preg Wrapped Split-Bonded Cylinders of Batch-1

\begin{tabular}{|c|c|c|c|c|c|c|c|}
\hline \multirow[t]{2}{*}{ FRP } & \multirow[t]{2}{*}{$\begin{array}{l}\text { No. of } \\
\text { Layers }\end{array}$} & Split in & $\begin{array}{l}\text { Axial } \\
\text { Strength }\end{array}$ & $\begin{array}{c}\text { Avg. } \\
\text { Axial } \\
\text { Strength }\end{array}$ & Stress & $\begin{array}{l}\text { Avg. } \\
\text { Stress }\end{array}$ & \multirow[t]{2}{*}{$\begin{array}{c}\text { Strength } \\
\text { Ratio }\end{array}$} \\
\hline & & Cylinder? & (kip) & (kip) & (ksi) & (ksi) & \\
\hline \multirow{12}{*}{ HFRP } & \multirow{3}{*}{2} & \multirow{3}{*}{$\mathrm{Y}$} & 237 & \multirow{3}{*}{257} & 8.38 & \multirow{3}{*}{9.08} & \multirow{3}{*}{ - } \\
\hline & & & 270 & & 9.55 & & \\
\hline & & & 263 & & 9.30 & & \\
\hline & \multicolumn{7}{|c|}{ Comparison with non-wrapped full cylinders } \\
\hline & 0 & $\mathrm{~N}$ & - & 154.28 & - & 5.46 & - \\
\hline & 2 & $\mathrm{Y}$ & - & 257 & - & 9.08 & 1.66 \\
\hline & \multicolumn{7}{|c|}{ Comparison with non-wrapped split-bonded cylinders } \\
\hline & 0 & $\bar{Y}$ & - & 111.33 & - & 3.94 & - \\
\hline & 2 & $\mathrm{Y}$ & - & 257 & - & 9.08 & 2.31 \\
\hline & \multicolumn{7}{|c|}{ Comparison with 2 layer wrapped full cylinders } \\
\hline & 2 & $\mathrm{~N}$ & - & 268 & - & 9.47 & - \\
\hline & 2 & $\mathrm{Y}$ & - & 257 & - & 9.08 & 0.96 \\
\hline
\end{tabular}

Note: N-No; Y-Yes

* The average stress obtained by the HFRP pre-preg wrapped split-bonded cylinders of batch-1 is 9.08 ksi against $9.47 \mathrm{ksi}$ of wrapped full cylinders.

* Strength recovery ratio of Table D-1 shows that HFRP pre-preg wrapped split-bonded cylinders of batch-1 has obtained $96 \%$ which is nearly the strength as wrapped full cylinders. 
Table D-2 Compressive Strength of CFRP Wrapped Split-Bonded Cylinders of Batch-2

\begin{tabular}{|c|c|c|c|c|c|c|c|}
\hline \multirow[t]{2}{*}{ FRP } & \multirow[t]{2}{*}{$\begin{array}{l}\text { No. of } \\
\text { Layers }\end{array}$} & Split in & $\begin{array}{l}\text { Axial } \\
\text { Strength }\end{array}$ & $\begin{array}{l}\text { Avg. } \\
\text { Axial } \\
\text { Strength }\end{array}$ & Stress & $\begin{array}{l}\text { Avg. } \\
\text { Stress }\end{array}$ & \multirow[t]{2}{*}{$\begin{array}{c}\text { Strength } \\
\text { Ratio }\end{array}$} \\
\hline & & Cylinder? & (kip) & (kip) & (ksi) & (ksi) & \\
\hline \multirow{12}{*}{ CFRP } & \multirow{3}{*}{2} & \multirow{3}{*}{ Y } & $328 *$ & \multirow{3}{*}{412} & 11.60 & \multirow{3}{*}{14.55} & \multirow{3}{*}{ - } \\
\hline & & & 423 & & 14.96 & & \\
\hline & & & 400 & & 14.15 & & \\
\hline & \multicolumn{7}{|c|}{ Comparison with non-wrapped full cylinders } \\
\hline & 0 & $\mathrm{~N}$ & - & 181 & - & 6.40 & - \\
\hline & 2 & $\mathrm{Y}$ & - & 412 & - & 14.55 & 2.27 \\
\hline & \multicolumn{7}{|c|}{ Comparison with non-wrapped split-bonded cylinders } \\
\hline & 0 & $\mathrm{Y}$ & - & 126 & - & 4.47 & - \\
\hline & 2 & Y & - & 412 & - & 14.55 & 3.26 \\
\hline & \multicolumn{7}{|c|}{ Comparison with 2 layer wrapped full cylinders } \\
\hline & 2 & $\mathrm{~N}$ & - & 437 & - & 15.46 & - \\
\hline & 2 & Y & - & 412 & - & 14.55 & 0.94 \\
\hline
\end{tabular}

Note: N-No; Y-Yes

* Outlier not considered in calculating average values

* The average stress obtained by the CFRP wrapped split-bonded cylinders of batch-2 is

$14.55 \mathrm{ksi}$ against $15.46 \mathrm{ksi}$ of wrapped full cylinders.

* Strength recovery ratio of Table D-2 shows that CFRP wrapped split-bonded cylinders of batch-2 has obtained $94 \%$ which is nearly the strength of wrapped full cylinders. 


\section{APPEndix E: BeAM Theoretical Calculation}

\section{Example 1:}

$>$ Four Point Bending on Externally Strengthened RC beam of Batch I with 2 layers of

\section{HFRP Pre-preg:}

Dimensions:

$b=6 \mathrm{in}$.

$h=15 \mathrm{in}$.

$d=13$ in.

$d^{\prime}=1.5 \mathrm{in}$.

$d^{\prime \prime}=2$ in.

\section{Reinforcement:}

No. of bars in tension $=2$ No.

No. of bars in compression $=2$ No.

$\phi_{\text {compression }}=3 / 8^{\prime \prime}$

$\phi_{\text {tension }}=3 / 8^{\prime \prime}$

$A_{s}=0.11 \mathrm{in}^{2}$

$A_{s}^{\prime}=0.11 \mathrm{in}^{2}$

Given:

$f_{c}^{\prime}=5.46 k s i$

$f_{y}=60 \mathrm{ksi}$

$f_{s}=60 k s i$

$f_{s}^{\prime}=60 k s i$

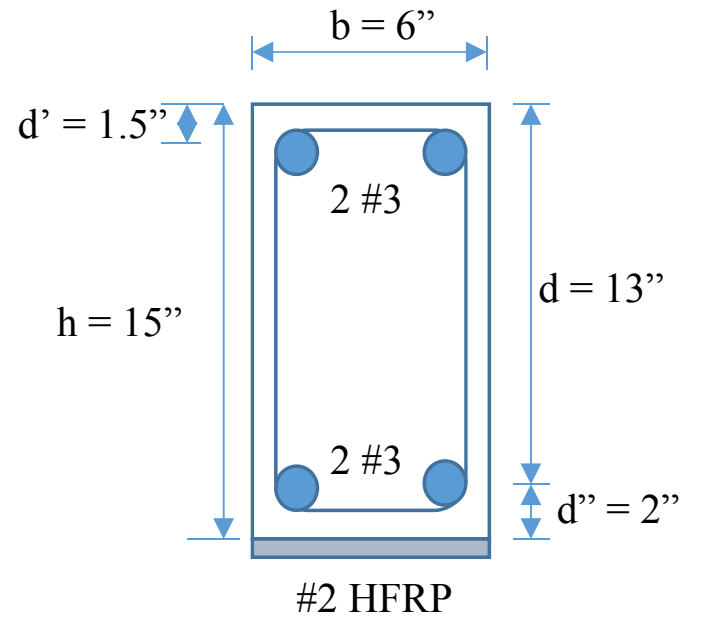

H2 HFRP 
$\beta_{1}=0.777$

Tensile Load of 2 layer HFRP coupon per unit width, $P_{T}=5540 \mathrm{lb}$

$E_{f r p}=8870 k s i$

$E_{\text {steel }}=29000 \mathrm{ksi}$

From equilibrium:

Total compression force $=$ Total tension force

$$
\begin{gathered}
\boldsymbol{C}_{\text {concrete }}=\boldsymbol{T}_{\text {steel }}+\boldsymbol{T}_{\text {frp }} \\
0.85 f_{c}^{\prime} \beta_{1} c b+A_{s}^{\prime} f_{s}^{\prime}=A_{s} f_{y}+A_{f r p} f_{f r p} \\
A_{f r p}=n t_{f} b \\
f_{\text {frp }}=\frac{P_{T}}{n t_{f}}
\end{gathered}
$$

Where,

$$
\begin{aligned}
& n=\text { number of layers }=2 \\
& t_{f}=\text { thickness of fabric for } 2 \text { layers }=0.036^{\prime \prime} \\
& C_{\text {concrete }}=0.85 * 5460 * 0.777 * c * 6+(0.11 * 2) * 60000 \\
& T=T_{\text {steel }}+\boldsymbol{T}_{\text {frp }}=(0.11 * 2) * 60000+(0.036 * 6) *\left(\frac{\mathbf{5 5 4 0}}{\mathbf{1} * 0.036}\right) \\
& c=1.54 \text { in } . \\
& a=\beta_{1} c=0.777 * 1.54=1.2 \mathrm{in} .
\end{aligned}
$$

Resisting moment of wrapped beam with 2 layers of HFRP pre-preg:

$$
\begin{aligned}
& M_{n}=\boldsymbol{T}_{\text {steel }}\left(d-\frac{a}{2}\right)+\boldsymbol{T}_{\text {frp }}\left(h-\frac{a}{2}\right) \\
& =13200\left(13-\frac{1.2}{2}\right)+33240\left(15-\frac{1.2}{2}\right)
\end{aligned}
$$




$$
\begin{gathered}
=624.34 \mathrm{kip}-\mathrm{in} . \\
=53.53 \mathrm{k}-\mathrm{ft}
\end{gathered}
$$

From four point bending test: $($ span $=9 \mathrm{ft})$

$$
\begin{aligned}
P_{n} & =M_{n} * \frac{6}{\operatorname{span}} \\
& =53.53 * \frac{6}{9} \\
& =35.69 \mathrm{kip}
\end{aligned}
$$

Hence, Maximum load and resisting moment from theory are 35.69 kip and $53.53 \mathrm{k-ft}$ respectively. Experimental values are about $60 \%$ to $90 \%$ of the theoretical values. Hence, conservatively $60 \%$ of the debonding strain values are considered for theoretical calculations.

With Debonding Strain Values:

$$
\begin{aligned}
\varepsilon_{f d} & =0.083 * \sqrt{f_{c}^{\prime} / n E_{f} t_{f}} \leq 0.9 * \varepsilon_{f u} \\
& =0.0109
\end{aligned}
$$

\section{From equilibrium:}

$$
\begin{gathered}
\text { Total compression force }=\text { Total tension force } \\
\boldsymbol{C}_{\text {concrete }}=\boldsymbol{T}_{\text {steel }}+\boldsymbol{T}_{\text {frp }} \\
0.85 f_{c}^{\prime} \beta_{1} c b+A_{s}^{\prime} f_{s}^{\prime}=A_{s} f_{y}+A_{f r p} f_{f r p} \\
A_{f r p}=n t_{f} b \\
f_{\text {frp }}=0.6 * \varepsilon_{d} * E_{\text {frp }}=0.6 * 0.0109 * 8870 * 1000=57761 p s i \\
\boldsymbol{C}_{\text {concrete }}=\mathbf{0 . 8 5} * \mathbf{5 4 6 0} * \mathbf{0 . 7 7 7} * \boldsymbol{c} * \mathbf{6}+(\mathbf{0 . 1 1} * \mathbf{2}) * \mathbf{6 0 0 0 0}
\end{gathered}
$$




$$
\begin{gathered}
T=T_{\text {steel }}+\boldsymbol{T}_{\text {frp }}=(0.11 * 2) * 60000+(0.036 * 6) *(57761) \\
c=0.89 \mathrm{in} . \\
a=\beta_{1} c=0.777 * 0.89=0.69 \mathrm{in} .
\end{gathered}
$$

Resisting moment of wrapped beam with 2 layers of HFRP pre-preg:

$$
\begin{gathered}
M_{n}=\boldsymbol{T}_{\text {steel }}\left(d-\frac{a}{2}\right)+\boldsymbol{T}_{\text {frp }}\left(h-\frac{a}{2}\right) \\
=13200\left(13-\frac{0.69}{2}\right)+12476\left(15-\frac{0.69}{2}\right) \\
=349.9 \mathrm{kip}-\mathrm{in} . \\
=29.16 \mathrm{k}-\mathrm{ft}
\end{gathered}
$$

Load Capacity: $(\operatorname{span}=9 \mathrm{ft})$

$$
\begin{aligned}
P_{n} & =M_{n} * \frac{6}{\text { span }} \\
& =29.16 * \frac{6}{9} \\
& =19.4 \mathrm{kip}
\end{aligned}
$$

Hence, Maximum load and resisting moment from theory are $19.4 \mathrm{kip}$ and $29.16 \mathrm{k}-\mathrm{ft}$ respectively. Experimental values are $83 \%$ of the theoretical values calculated for $60 \%$ of the effective debonding strain values.

\section{Example 2:}

Four Point Bending on Externally Strengthened RC beam of Batch I with 2 layers of CFRP-epoxy:

Dimensions:

$b=6 \mathrm{in}$. 
$h=15$ in.

$d=13$ in.

$d^{\prime}=1.5$ in.

$d^{\prime \prime}=2$ in.

\section{Reinforcement:}

No. of bars in tension $=2$ No.

No. of bars in compression $=2$ No.

$\phi_{\text {compression }}=3 / 8^{\prime \prime}$

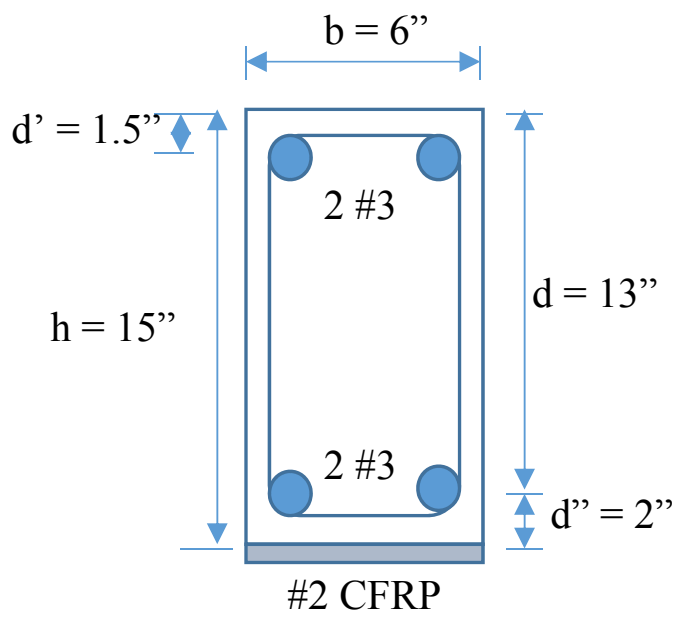

$\phi_{\text {tension }}=3 / 8^{\prime \prime}$

$A_{s}=0.11 \mathrm{in}^{2}$

$A_{s}^{\prime}=0.11 \mathrm{in}^{2}$

\section{Given:}

$f_{c}^{\prime}=5.46 k s i$

$f_{y}=60 \mathrm{ksi}$

$f_{s}=60 \mathrm{ksi}$

$f_{s}^{\prime}=60 k s i$

$\beta_{1}=0.777$

Tensile Load of 2 layer HFRP coupon per unit width, $P_{T}=7440$ lb

$E_{\text {frp }}=14000 \mathrm{ksi}$

$E_{\text {steel }}=29000 \mathrm{ksi}$

From equilibrium:

Total compression force $=$ Total tension force

$$
C_{\text {concrete }}=T_{\text {steel }}+T_{\text {frp }}
$$




$$
\begin{gathered}
0.85 f_{c}^{\prime} \beta_{1} c b+A_{s}^{\prime} f_{s}^{\prime}=A_{s} f_{y}+A_{f r p} f_{f r p} \\
A_{f r p}=n t_{f} b \\
f_{f r p}=\frac{P_{T}}{n t_{f}}
\end{gathered}
$$

Where,

$$
\begin{aligned}
& n=\text { number of layers }=2 \\
& t_{f}=\text { thickness of fabric for } 2 \text { layers }=0.052^{\prime \prime} \\
& \boldsymbol{C}_{\text {concrete }}=\mathbf{0 . 8 5} * \mathbf{5 4 6 0} * \mathbf{0 . 7 7 7} * \boldsymbol{c} * \mathbf{6}+(\mathbf{0 . 1 1} * \mathbf{2}) * \mathbf{6 0 0 0 0} \\
& \boldsymbol{T}=\boldsymbol{T}_{\text {steel }}+\boldsymbol{T}_{\text {frp }}=(\mathbf{0 . 1 1} * \mathbf{2}) * \mathbf{6 0 0 0 0}+(\mathbf{0 . 0 5 2} * \mathbf{6}) *\left(\frac{\mathbf{7 4 4 0}}{\mathbf{1} * \mathbf{0 . 0 5 2}}\right) \\
& \boldsymbol{c}=\mathbf{2 . 0 6} \mathrm{in} . \\
& \boldsymbol{a}=\boldsymbol{\beta}_{\mathbf{1}} \boldsymbol{c}=\mathbf{0 . 7 7 7} * \mathbf{2 . 0 6}=\mathbf{1 . 6 0 1} \mathrm{in} .
\end{aligned}
$$

Resisting moment of wrapped beam with 2 layers of HFRP pre-preg:

$$
\begin{gathered}
M_{n}=\boldsymbol{T}_{\text {steel }}\left(d-\frac{a}{2}\right)+\boldsymbol{T}_{\text {frp }}\left(h-\frac{a}{2}\right) \\
=13200\left(13-\frac{1.601}{2}\right)+44640\left(15-\frac{1.601}{2}\right) \\
=794.9 \mathrm{kip}-\mathrm{in} . \\
=66.24 \mathrm{k}-\mathrm{ft}
\end{gathered}
$$

From four point bending test: $(\operatorname{span}=9 \mathrm{ft})$

$$
\begin{aligned}
P_{n} & =M_{n} * \frac{6}{\text { span }} \\
& =66.24 * \frac{6}{9} \\
& =44.16 \mathrm{kip}
\end{aligned}
$$


Hence, Maximum load and resisting moment from theory are 44.16 kip and $66.24 \mathrm{k}-\mathrm{ft}$ respectively. Experimental values are about $45 \%$ to $60 \%$ of the theoretical values. Hence, conservatively $60 \%$ of the debonding strain values are considered for theoretical calculations.

\section{With Debonding Strain Values:}

$$
\begin{aligned}
\varepsilon_{f d} & =0.083 * \sqrt{f_{c}^{\prime} / n E_{f} t_{f}} \leq 0.9 * \varepsilon_{f u} \\
& =0.0072
\end{aligned}
$$

From equilibrium:

$$
\begin{gathered}
\text { Total compression force }=\text { Total tension force } \\
\boldsymbol{C}_{\text {concrete }}=\boldsymbol{T}_{\text {steel }}+\boldsymbol{T}_{\text {frp }} \\
0.85 f_{c}^{\prime} \beta_{1} c b+A_{s}^{\prime} f_{s}^{\prime}=A_{s} f_{y}+A_{\text {frp }} f_{\text {frp }} \\
A_{\text {frp }}=n t_{f} b \\
f_{\text {frp }}=\varepsilon_{\text {frp }} * E_{\text {frp }}=0.6 * 0.0072 * 14000 * 1000=60379 p s i \\
\boldsymbol{C}_{\text {concrete }}=\mathbf{0 . 8 5} * \mathbf{5 4 6 0} * \mathbf{0 . 7 7 7} * \boldsymbol{c} * \mathbf{6}+(\mathbf{0 . 1 1} * \mathbf{2}) * \mathbf{6 0 0 0 0} \\
\boldsymbol{T}=\boldsymbol{T}_{\text {steel }}+\boldsymbol{T}_{\boldsymbol{f r p}}=(\mathbf{0 . 1 1} * \mathbf{2}) * \mathbf{6 0 0 0 0}+(\mathbf{0 . 0 5 2} * \mathbf{6}) *(\mathbf{6 0 3 7 9}) \\
\boldsymbol{c}=\mathbf{0 . 8 7} \mathbf{i n} . \\
\boldsymbol{a}=\boldsymbol{\beta}_{\mathbf{1}} \boldsymbol{c}=\mathbf{0 . 7 7 7} * \mathbf{0 . 8 7}=\mathbf{0 . 6 8} \mathbf{i n} .
\end{gathered}
$$

Resisting moment of wrapped beam with 2 layers of HFRP pre-preg:

$$
\begin{aligned}
& M_{n}=\boldsymbol{T}_{\text {steel }}\left(d-\frac{a}{2}\right)+\boldsymbol{T}_{\boldsymbol{f r p}}\left(h-\frac{a}{2}\right) \\
& =13200\left(13-\frac{0.65}{2}\right)+18838\left(15-\frac{0.68}{2}\right)
\end{aligned}
$$




$$
\begin{gathered}
=442.51 \mathrm{kip}-\mathrm{in} . \\
=36.88 \mathrm{k}-\mathrm{ft}
\end{gathered}
$$

Load Capacity: $(\operatorname{span}=9 \mathrm{ft})$

$$
\begin{aligned}
P_{n} & =M_{n} * \frac{6}{\operatorname{span}} \\
& =36.88 * \frac{6}{9} \\
& =24.58 \mathrm{kip}
\end{aligned}
$$

Hence, Maximum load and resisting moment from theory are 24.58 kip and $36.88 \mathrm{k}-\mathrm{ft}$ respectively. Experimental values are $103 \%$ of the theoretical values calculated for $60 \%$ of the effective debonding strain values. 


\section{APPENDIX F: LOAD-DEFLECTION CURVES}

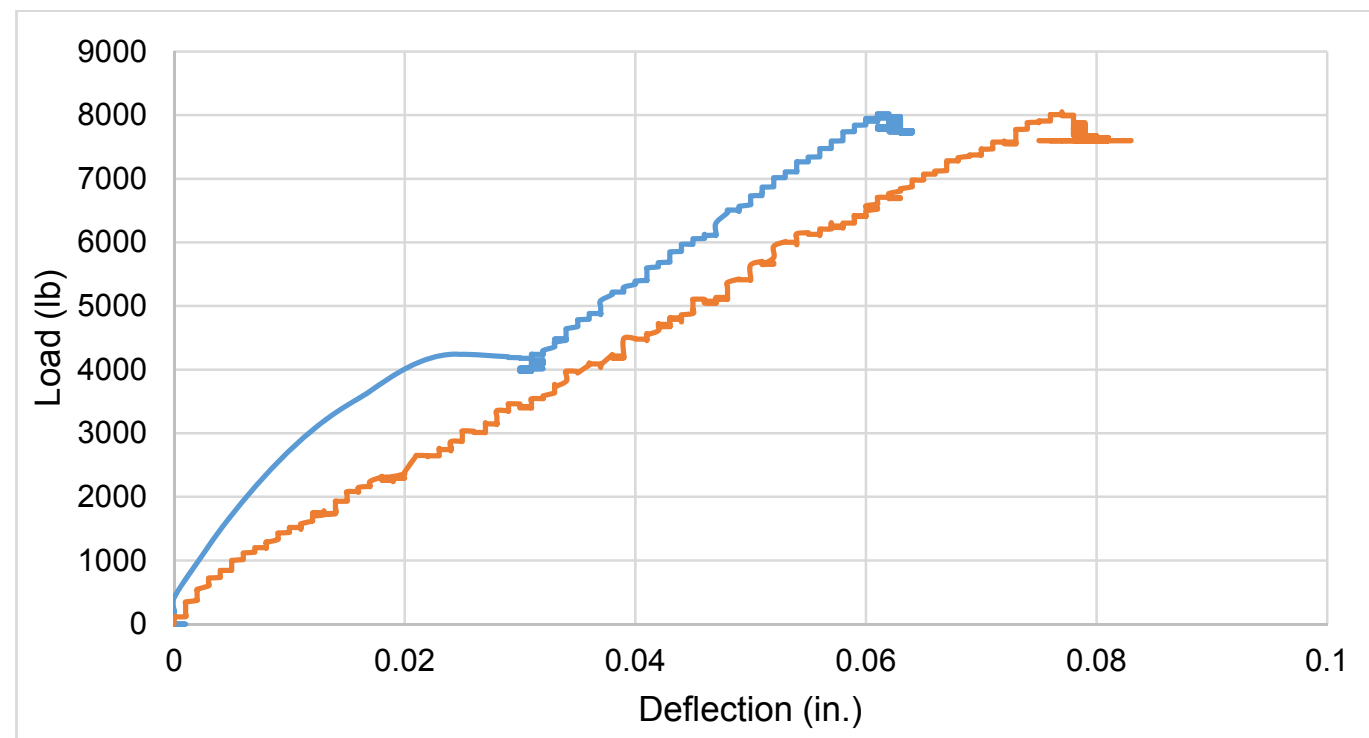

-B2_CFRP_2 layers_After Crack Injection —B2_CFRP_2 layers_Before Crack Injection

Figure F-1 Load-Deflection Curves for CFRP wrapped beam of Batch-2: Before Crack Injection v/s After Crack Injection

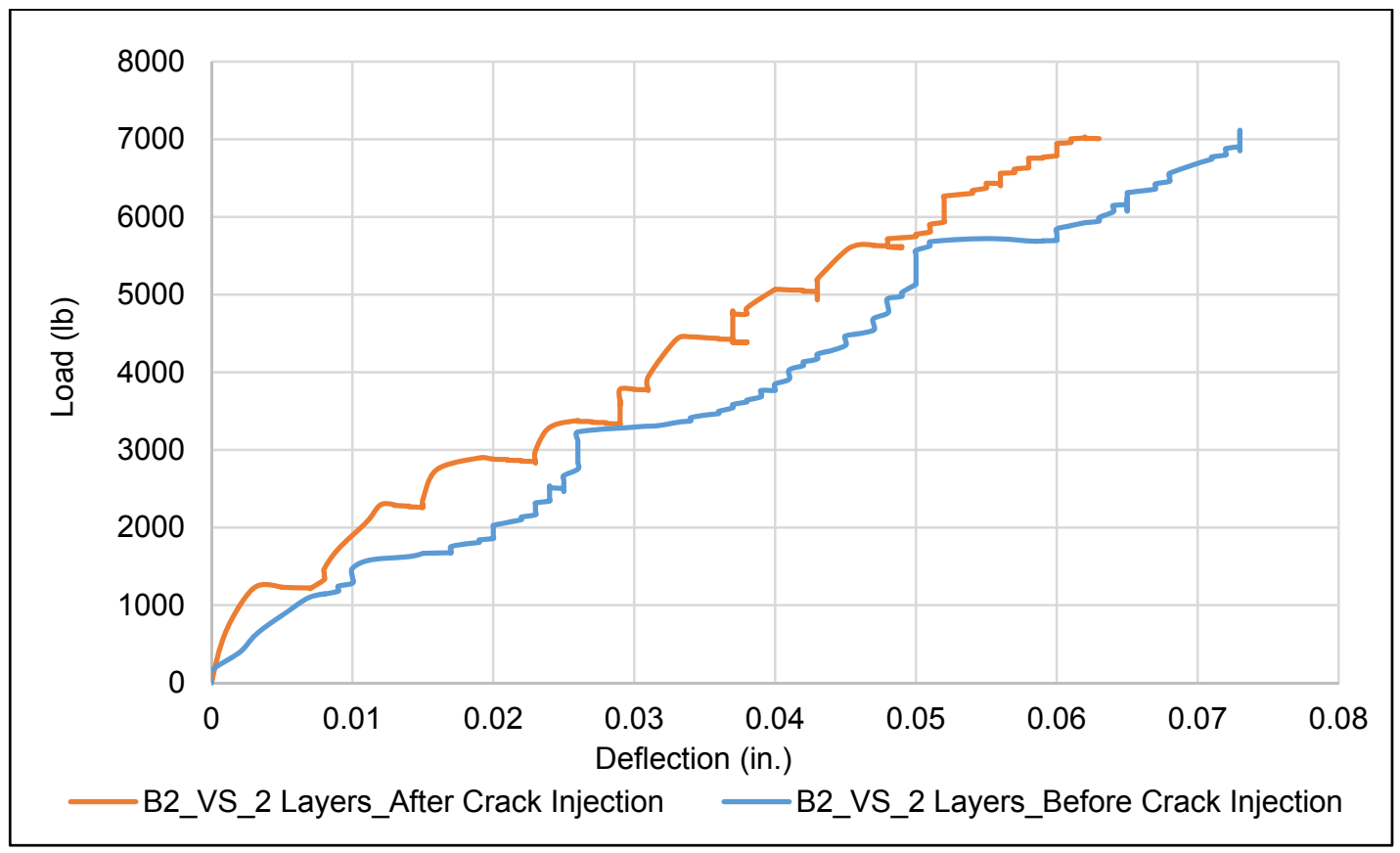

Figure F-2 Load-Deflection Curves for HFRP wrapped beam of Batch-2: Before Crack Injection v/s After Crack Injection 


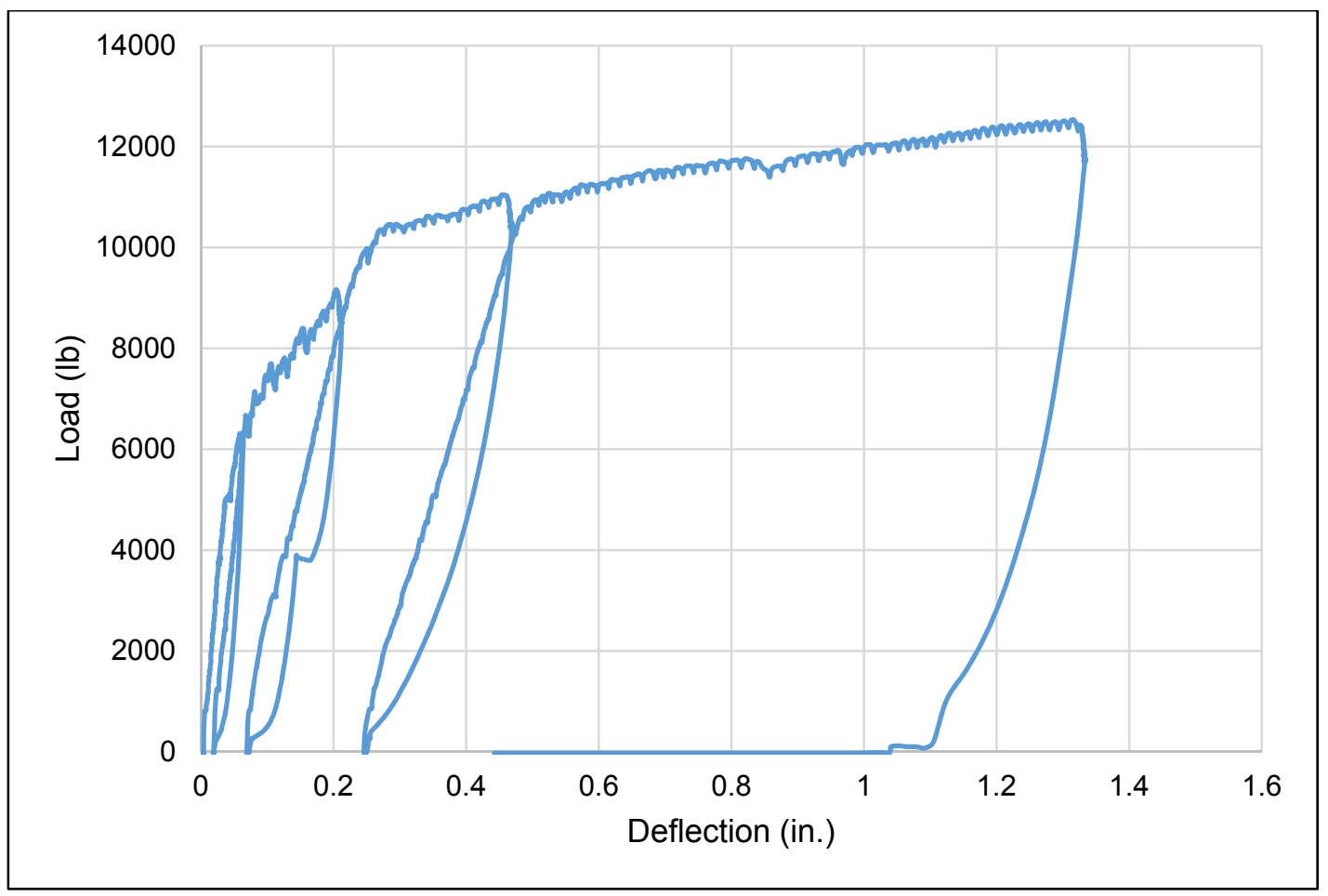

Figure F-3 Load-Deflection Curves for Control Beam of Batch-1

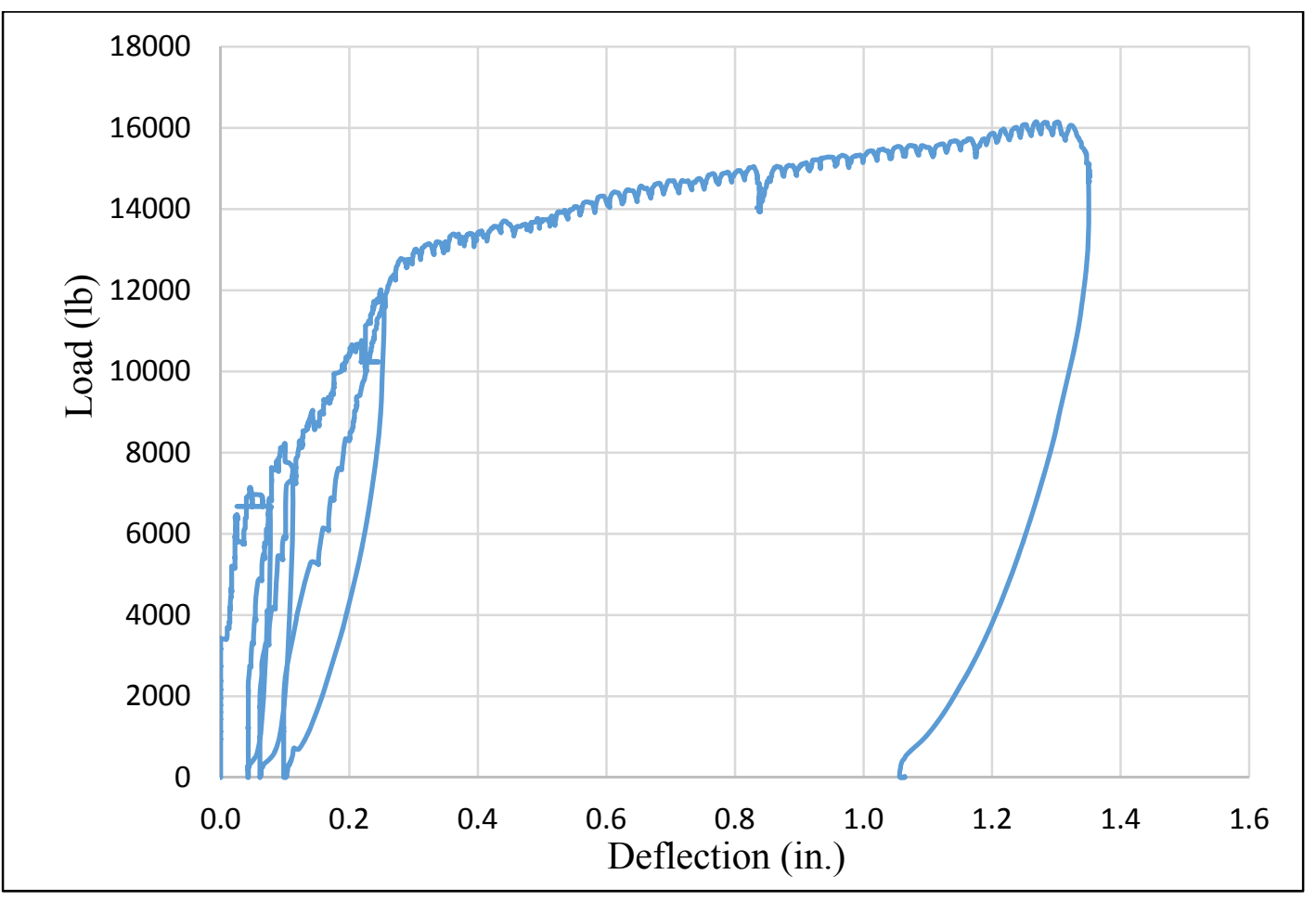

Figure F-4 Load-Deflection Curves for Control Beam-1 of Batch-2 


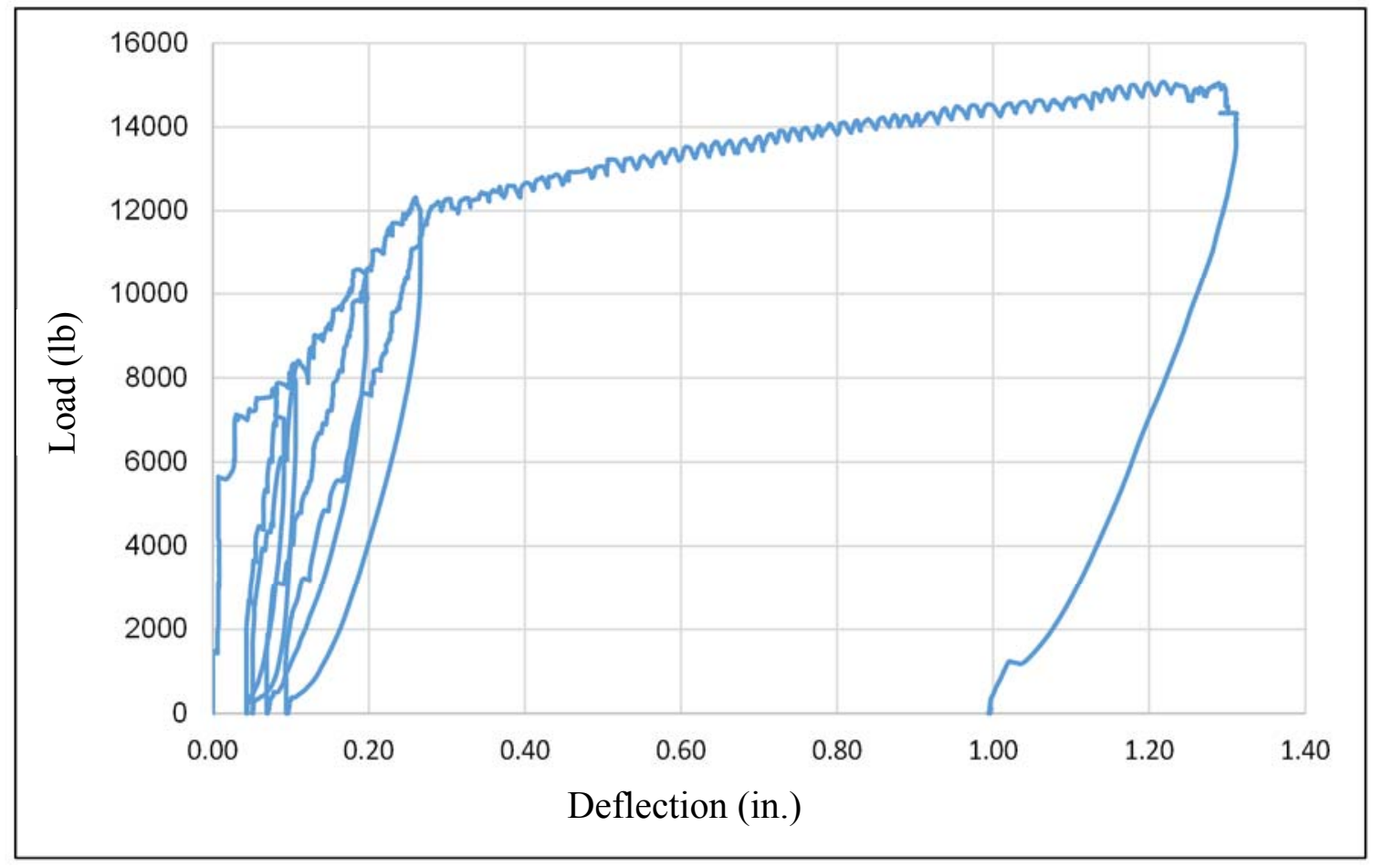

Figure F-5 Load-Deflection Curves for Control Beam-2 of Batch-2

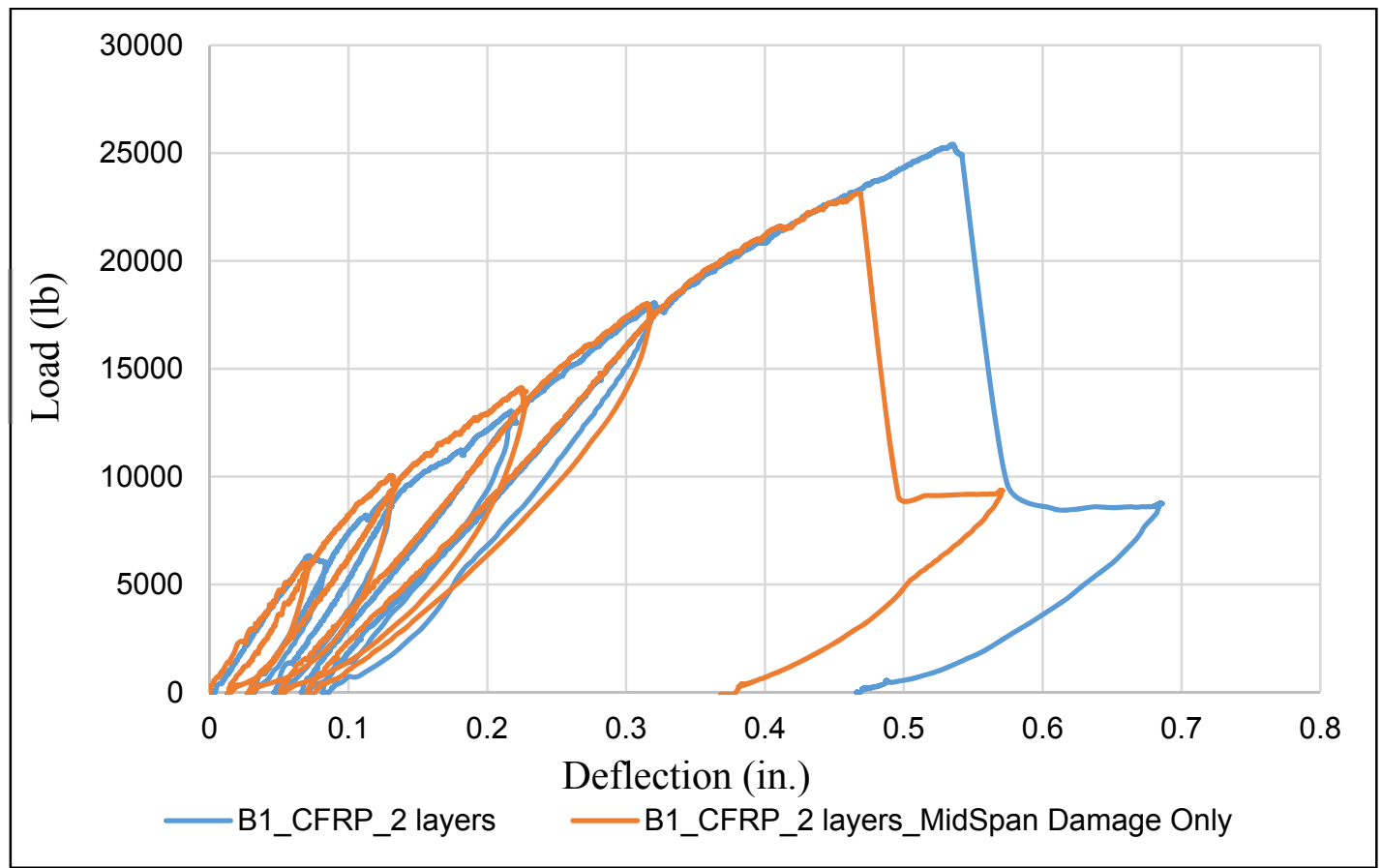

Figure F-6 Load-Deflection Curves for CFRP Wrapped Beams of Batch-1 


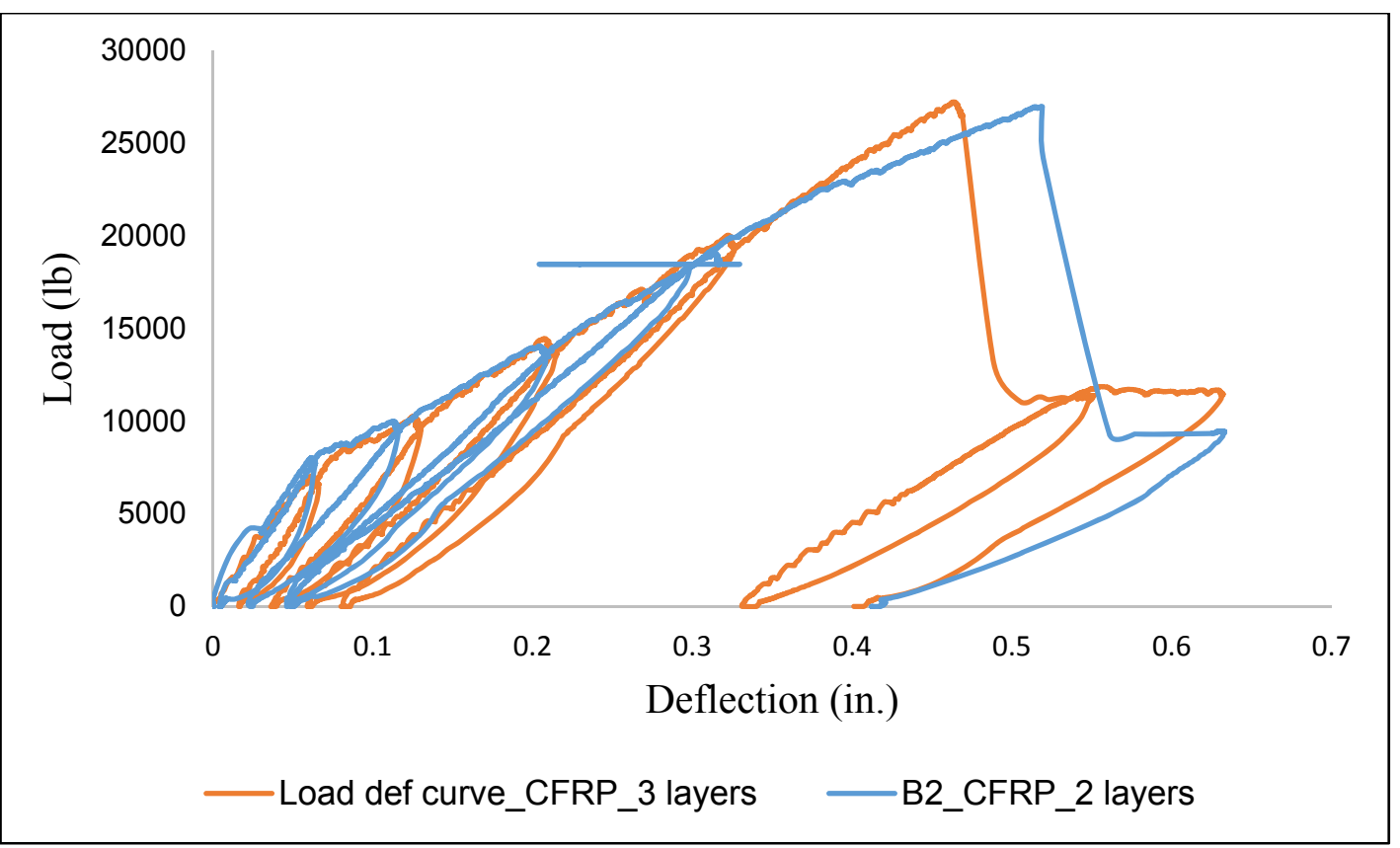

Figure F-7 Load-Deflection Curves for CFRP Wrapped Beams of Batch-2

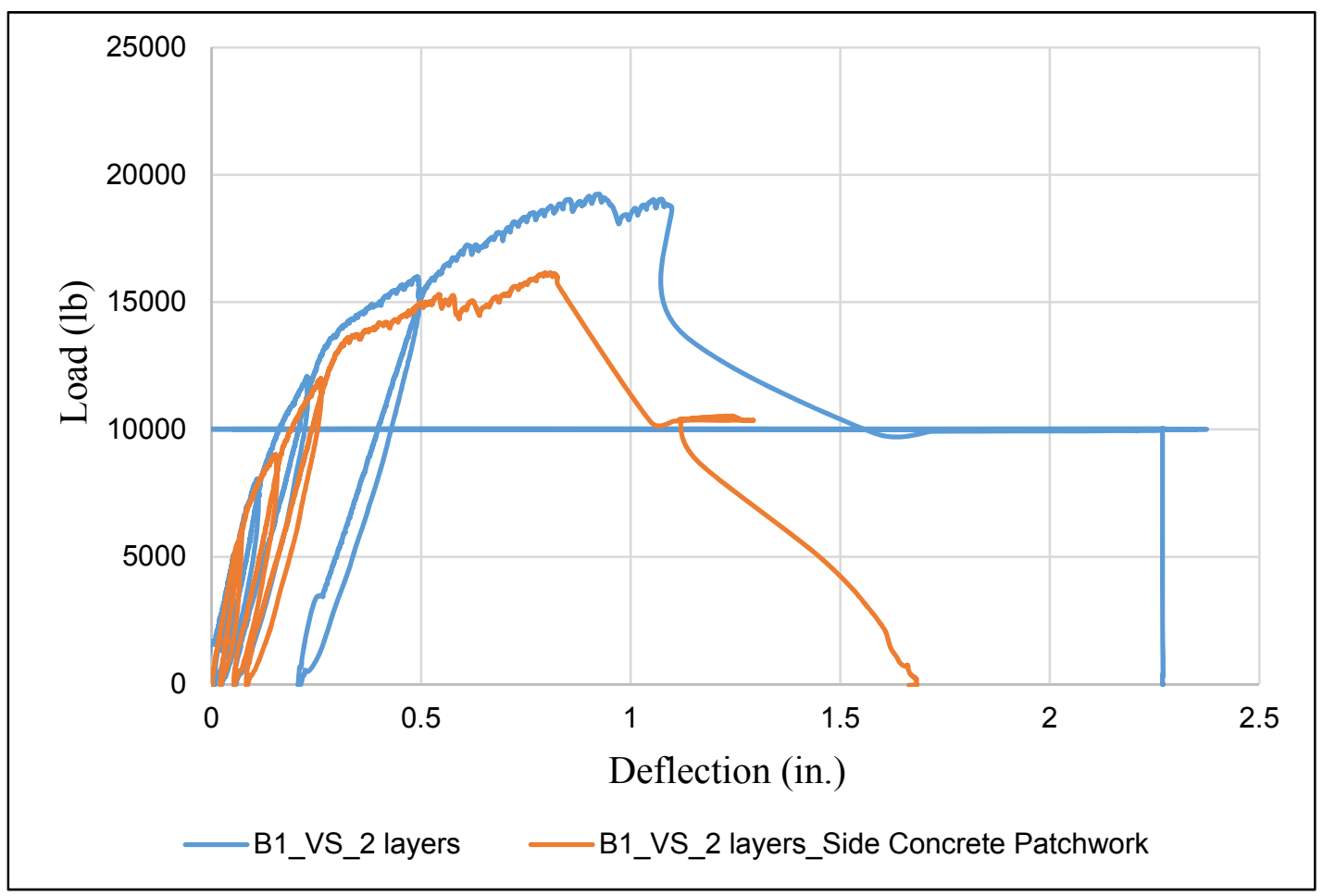

Figure F-8 Load-Deflection Curves for HFRP Wrapped Beams of Batch-1 


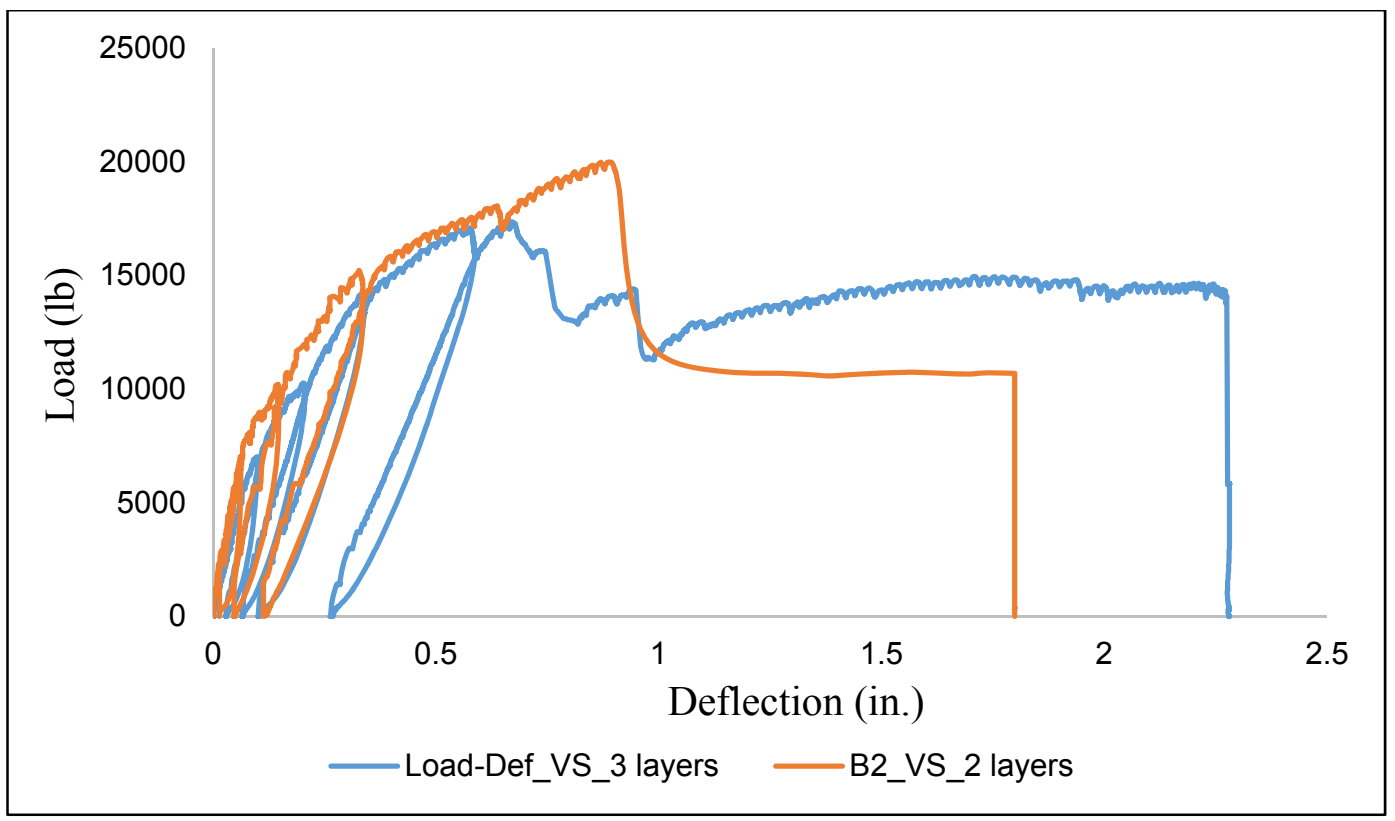

Figure F-9 Load-Deflection Curves for HFRP Wrapped Beams of Batch-2 